\title{
Scale-down of CHO Cell Fed-batch Cultures
}

Xiao Pan

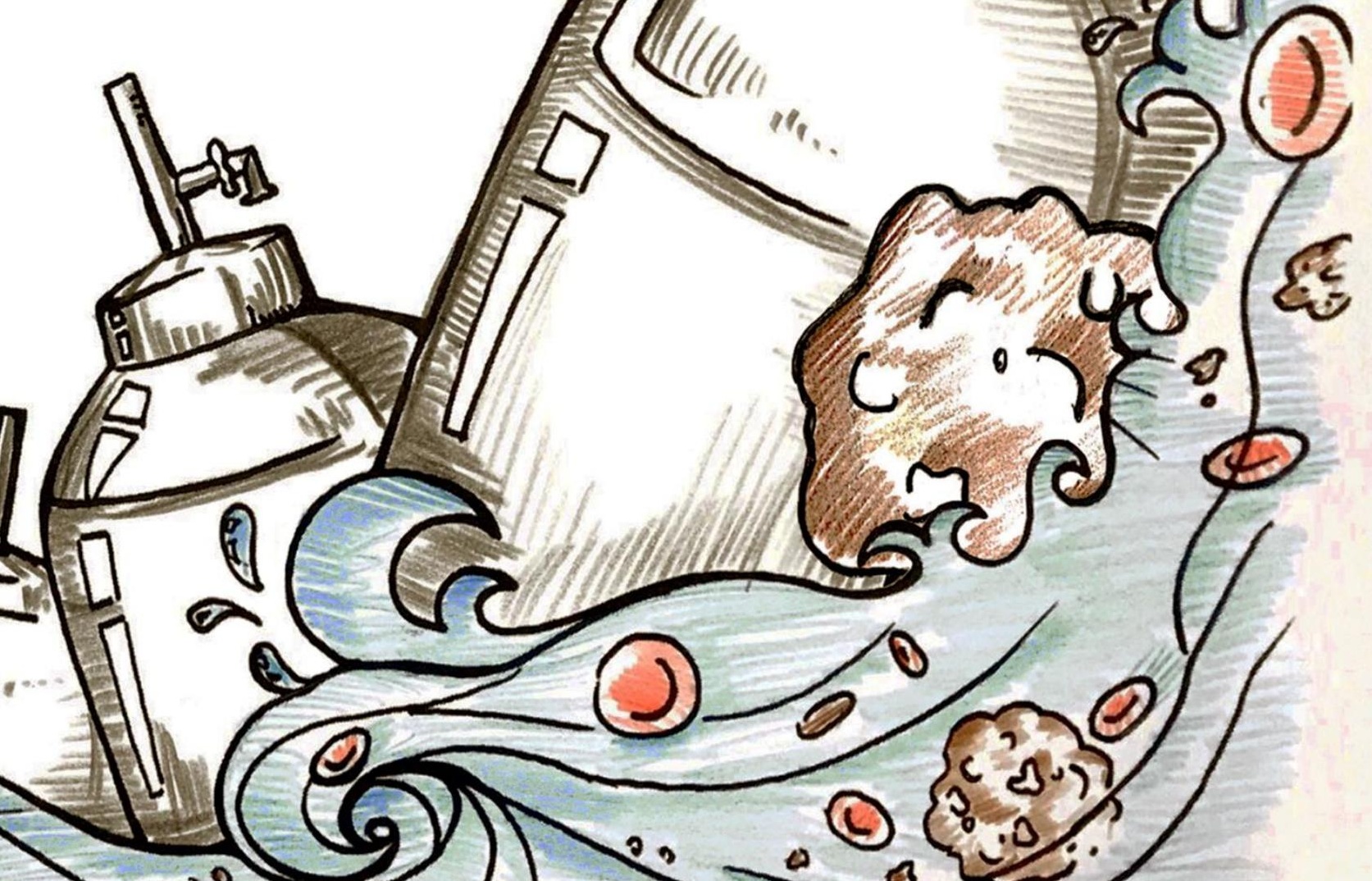



Scale-down of CHO Cell

Fed-batch Cultures

\author{
Xiao Pan
}




\section{Thesis committee}

\section{Promotor}

Prof. Dr R.H. Wijffels

Professor of Bioprocess Engineering

Wageningen University \& Research

\section{Co-promotors}

Dr D.E. Martens

Associate professor, Bioprocess Engineering Group

Wageningen University \& Research

Dr M.C.F. Dalm

Lead Scientist, Synthon Biopharmaceuticals BV, Nijmegen, the Netherlands

\section{Other members}

Prof. Dr D. Weijers, Wageningen University \& Research

Prof. Dr F. Hesse, Institute for Applied Biotechnology, Biberach, Germany

Dr L. Boon, Bioceros, Utrecht, the Netherlands

Dr G. Zijlstra, Sartorius Stedim Biotech GmbH, Groningen, the Netherlands

This research was conducted under the auspices of the Graduate School VLAG (Advanced studies in Food Technology, Agrobiotechnology, Nutrition and Health Sciences) 


\title{
Scale-down of CHO Cell Fed-batch Cultures
}

\author{
Xiao Pan
}

\section{Thesis}

submitted in fulfilment of the requirements for the degree of doctor at Wageningen University

by the authority of the Rector Magnificus,

Prof. Dr A.P.J. Mol,

in the presence of the

Thesis Committee appointed by the Academic Board

to be defended in public

on Tuesday 29 May 2018

at 1:30 p.m. in the Aula. 
Xiao Pan

Scale-down of CHO Cell Fed-batch Cultures, 157 pages.

PhD thesis, Wageningen University, Wageningen, the Netherlands (2018) With references, with summary in English

ISBN 978-94-6343-843-8

DOI https://doi.org/10.18174/444363 
Dedicated to Yimin and Muyun, 我的大小情人 



\section{Table of Contents}

$\begin{array}{lr}\text { Chapter } 1 & 9\end{array}$

Introduction

Chapter 2

Selection of chemically defined media for $\mathrm{CHO}$ cell fed-batch culture processes

Chapter 3

Metabolic characterization of a $\mathrm{CHO}$ cell size increase phase in fed-batch cultures

Chapter 4

Transcriptome analysis of $\mathrm{CHO}$ cell size increase during a fed-batch process

Chapter 5

Transcriptome analysis for the scale-down of a $\mathrm{CHO}$ cell fed-batch process

Chapter 6

General discussion 

Chapter 1

Introduction and thesis outline 



\subsection{Antibody production by mammalian cells}

Therapeutic proteins are an important class of medicines for treating diseases such as different types of cancer ${ }^{1}$. Among these proteins, monoclonal antibodies (mAbs) are an important and rapidly growing class with total sales of $\$ 85$ billion in $2015^{2,3}$. A monoclonal antibody can specifically bind to antigens expressed by tumor cells and therefore, can be used to effectively target pathways important for treatment of malignancies ${ }^{4,5}$. Monoclonal antibodies are large and complex molecules (Figure 1). Their biosynthesis requires specific post-translational modifications which are very important for the protein efficacy, safety, and stability. In this regard, mammalian cells are used instead of other expression systems, such as bacteria and yeast, due to the ability to produce proteins with proper folding and post-translational modifications (e.g. glycosylation). However, mammalian cells have lower growth and production rates compared to bacterial and yeast cells. Furthermore, mammalian cells have complex nutrient requirements ${ }^{6-8}$ and are sensitive to culture conditions ${ }^{9-12}$. Such characteristics challenge the development of mammalian cell culture processes.

Of all the types of mammalian cells used for pharmaceutical protein production (e.g. HEK, PER.C6, and hybridoma cells), Chinese Hamster Ovary (CHO) cells are the most well studied. $\mathrm{CHO}$ cells are used for nearly $70 \%$ of all recombinant protein therapeutics produced ${ }^{1}$. A lot of progress has been made during the past decades regarding cell line, culture medium, and culture process development. This has resulted in an improvement of product titers with more than a factor 100 to the current level of grams/L, compared to $50 \mathrm{mg} / \mathrm{L}$ in the $1980 \mathrm{~s}^{13}$. Currently, the market demand for $\mathrm{mAb}$-based products is still increasing at a fast pace. In order to meet the market demand, efforts are being made to further increase the product yield of a culture process as well as to shorten the timeline for process development.

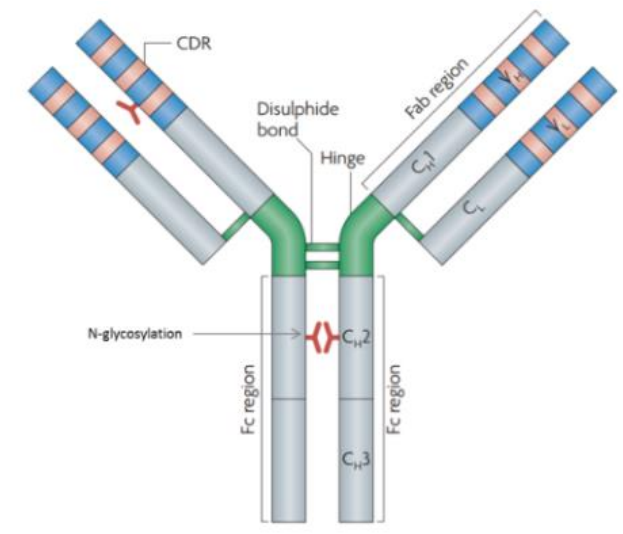

Figure 1. The representative structure of a monoclonal antibody. Fc, fragment crystallizable region; Fab, fragment antigen-binding region; CDR, complementarity-determining region; $C_{H}$, heavy chain constant domain; $C_{L}$, light chain constant domain; $V_{H}$, heavy chain variable domain; $V_{L}$, light chain variable domain. Figure adopted from Beck, A. et al. (2010) ${ }^{14}$ with permission from the author published in this thesis. 


\subsection{Production processes}

Several culture processes are currently being used in industry for $\mathrm{CHO}$ cell culture. These processes are (i) batch process: cells are cultured in a basal medium with no addition of nutrients during the culture time. The product is harvested after the cell viability starts dropping. This type of process is generally used during the preculture phase where cells are generated for inoculation of the production bioreactor. (ii) Fed-batch process: the culture starts in batch process mode, but the consumed nutrients are fed to the cells either continuously or as a bolus. In this way, the cells can grow to a higher concentration and can be maintained viable for a longer period of time, which together result in a higher product titer at the end of the process. (iii) Perfusion process: cells are retained in the reactor while being perfused constantly with fresh medium. In this way, the spent medium which contains inhibitory compounds produced by the cells are removed. This process typically results in a very high biomass concentration in the bioreactor and thus a high volumetric productivity.

Amongst these culture processes, fed-batch (as exemplified in Figure 2) is currently the most common process for the production of monoclonal antibodies in industry, thanks to the fact that it is easy to operate, robust, and flexible. Hence, this thesis focuses on the fed-batch process. Considerable progress has been made during the past decades on the fed-batch process for $\mathrm{CHO}$ cell cultures. They include designing and tailoring of medium and feed composition as well as the feeding strategy $y^{8,15-17}$ for specific $\mathrm{CHO}$ cell types, optimizing culture parameters ${ }^{18-20}$, and designing bioreactors ${ }^{21-24}$. Many metabolic studies have been carried out in order to gain mechanistic insights into cell physiology during the fed-batch processes. These studies measured the change of extracellular nutrients as well as the change of biomass composition during the process and combined them with the existing metabolic networks to calculate the intracellular metabolic fluxes. Furthermore, by incorporating isotopic labeling in metabolic flux analysis, more detailed information on the flux distribution was obtained ${ }^{25}$. More recently, with the advances of system biology tools and the publication of the $\mathrm{CHO}$ genome ${ }^{26}$, omics' studies (e.g. transcriptomics, proteomics, and metabolomics) have been increasingly applied in cell culture process development to identify genes related to recombinant protein productivity ${ }^{27-29}$, and to study different culture conditions that affect the culture performance ${ }^{19,30-32}$. These studies have contributed to the fundamental understanding of $\mathrm{CHO}$ cell metabolism and resulted in improved process performances. 

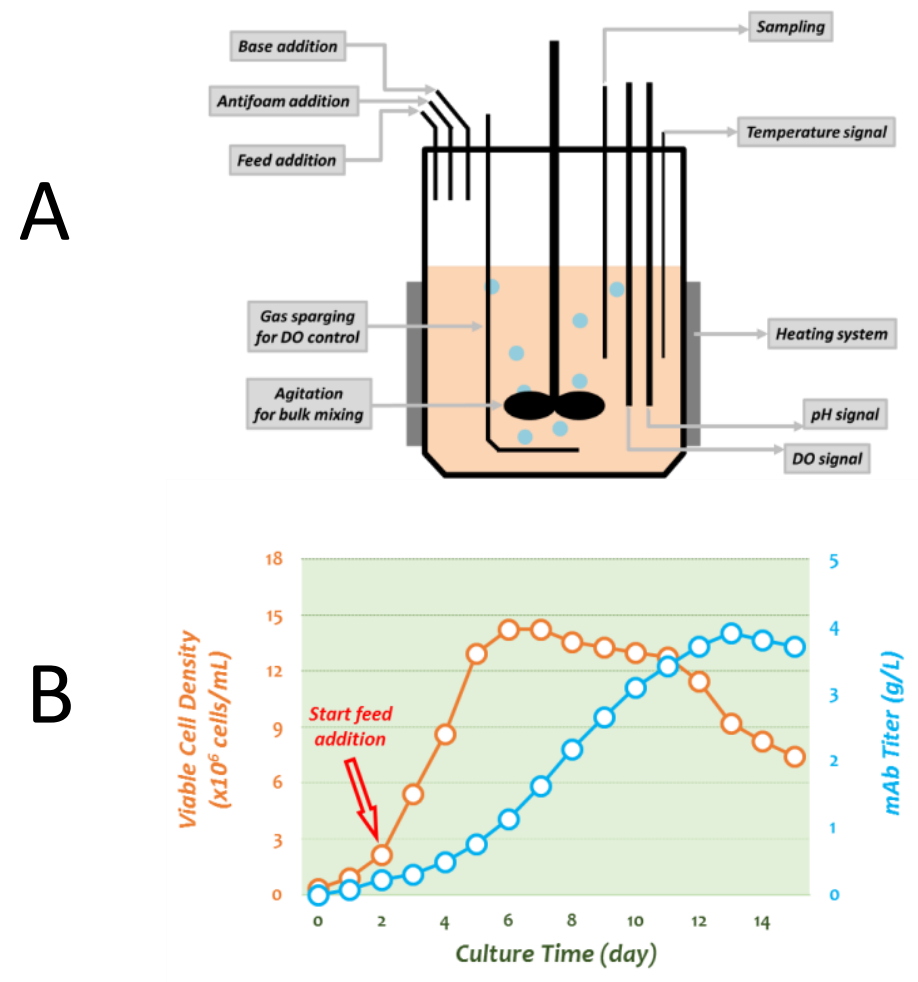

Figure 2. (A) Fed-batch culture process in a stirred tank bioreactor. (B) An example of typical cell growth and $m A b$ production profiles in a fed-batch process.

\subsection{Process development and scale-up}

A cell culture production process is developed in laboratory scale $(\leq 10 \mathrm{~L})$ bioreactors and is scaled up to large scale (e.g. kL-scale) for commercial production. The main steps comprise of cell line generation and selection, media and feed screening, process parameter determination, robustness evaluation, and scale-up to generate material for clinical trials and subsequent commercial production at the production scale (Figure 1.3). The work described in this thesis which is related to the tasks during the upstream process development is indicated in Figure 3. Different types of culture systems which are used in different steps during process development are shown in Figure 4. 


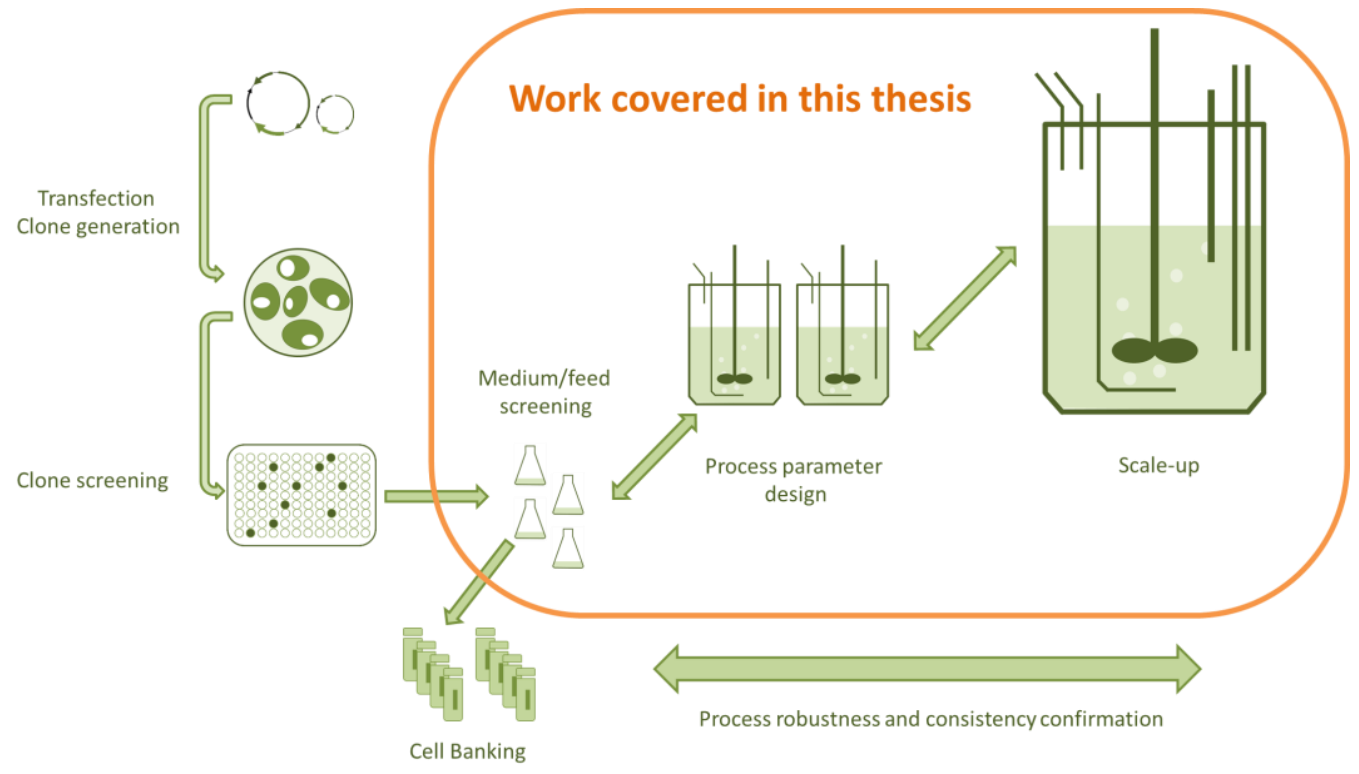

Figure 3. Main steps in upstream process development of mammalian cell cultures.

Upstream process development starts with clone generation and selection. This is typically done in microtiter plates and spin tubes with culture volumes ranging from $\mu \mathrm{L}$ to a few $\mathrm{mL}^{33,34}$. Up to thousands of clones and sub-clones, which harbor recombinant genes encoding the protein of interest, are generated in this step. After several rounds of screening, a few top productive clone candidates are selected for the subsequent step being medium and feed screening. In this step, various medium/feed systems are tested on the selected clones and this is typically done in shake flasks with a culture volume up to $100 \mathrm{~mL}$. The medium for mammalian cell culture has a complex formulation which is composed of more than 80 compounds that are needed for cell growth and production ${ }^{35}$. Various media systems are available for $\mathrm{CHO}$ cell culture and the formulations of these media differ ${ }^{8}$. As a result, a cell line can have a distinct performance in different media systems, which makes media selection an important step. Normally, media systems that support high product titers will be selected. Up to this step, the cells are cultured in an "uncontrolled" condition with regard to dissolved oxygen (DO) and pH. Next, the selected culture platforms (e.g. several clones and medium/feed candidates) will enter bench-scale bioreactors ( 1 to $10 \mathrm{~L}$ ) to examine the performance of the culture process under $\mathrm{pH}$ and DO controlled conditions. In this step, the number of clone and medium candidates will be further narrowed down. One or two selected processes will be further developed and optimized in the bench-scale bioreactors using the Design of Experiment (DoE) approach in order to find the optimal culture conditions for maximal $\mathrm{mAb}$ production. Typical culture conditions to be tested in this step include $\mathrm{DO}, \mathrm{pH}$, agitation rate, feeding rate, seeding density, and temperature. Once the process is more or less defined, the generated product can be used for product quality studies and pre-clinical trials. In the meantime, a process characterization study is carried out to better understand the process and to further 
define the process. In such a study, a range of culture parameters around the set point will be tested in order to define the acceptable ranges of these parameters. Operating the process within these ranges ensures a good product quality and productivity. Finally, the process design space is defined for the filing of the product, before further scale-up to a production scale $(\sim \mathrm{kL})$ is carried out. The time frame from clone generation to a manufacturing run varies for different projects, but typically takes one to two years.

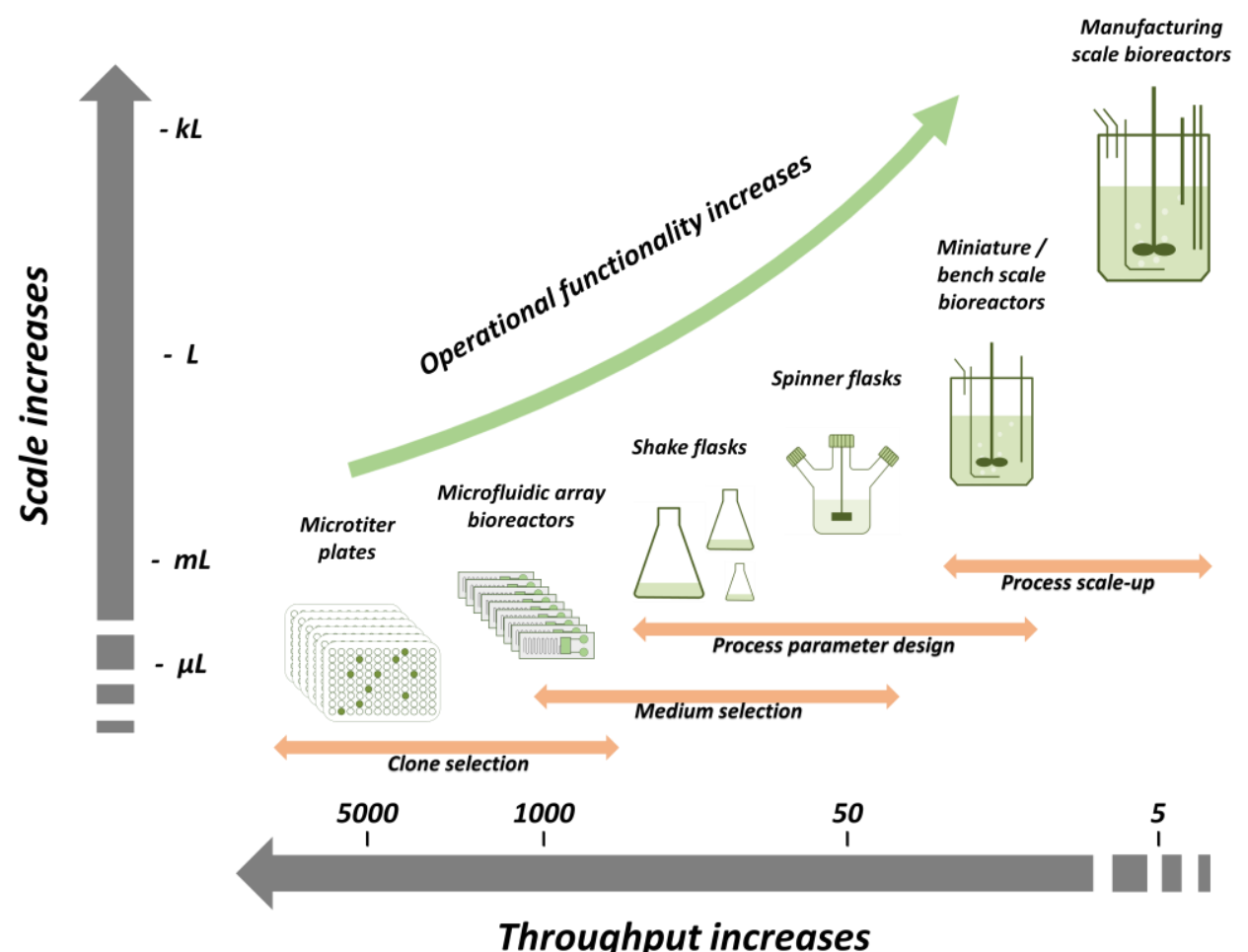

Figure 4. Typical cell culture systems used during process development.

Scale-up is an important step during the upstream development of mammalian cell culture processes. Unacceptable changes in process performance during the process scale-up means a lot of additional research into the causes, which is labor intensive and $\operatorname{costly}^{36}$. It is therefore very important that the culture system at the laboratory scale (scale-down system) is representative for the large scale. In this way, the design space can be translated one to one to the large scale. This is, however, not a straightforward task. The mass transfer (e.g. gas-liquid transfer and bulk mixing) and shear (from agitation and sparging) phenomena in a reactor are strongly dependent on the volume (scale) of the reactor. This makes it impossible to reproduce the same culture condition in a different scale, which may result in a different culture performance. In order to develop a representative scale-down process, the critical parameters which influence the process performance should be kept as much as possible the same between scales while allowing differences for the non-critical parameters. A lot of experience has been gained on how to keep critical parameters within acceptable ranges during the past decades ${ }^{37-40}$. Nevertheless, differences in 
process performance such as the maximum cell density and volumetric productivity are still sometimes observed between large scale and laboratory scale ${ }^{41-43}$. It is not always clear how scale differences lead to differences in process performance. Therefore, to improve scale-up/down, a better understanding on how the different conditions present at the different scales influence process performance is needed. For this, a good understanding of the relation between cell physiology and the specific process conditions is required.

\subsection{Aim and thesis outline}

The aim of this thesis is to obtain a better understanding of cell physiology in fedbatch cultures and on the scale-down of these processes. In the end, this knowledge should increase the efficiency of process development and thus shorten the time-tomarket for the biopharmaceutical products. The different scales used in this thesis and the associated culture systems are exemplified in Figure 5.

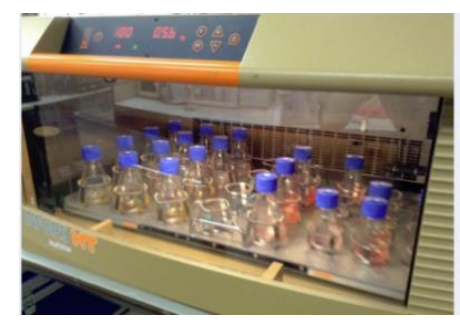

A

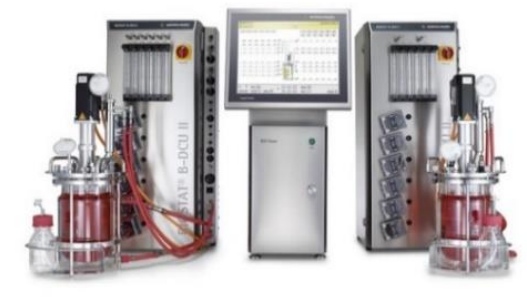

B

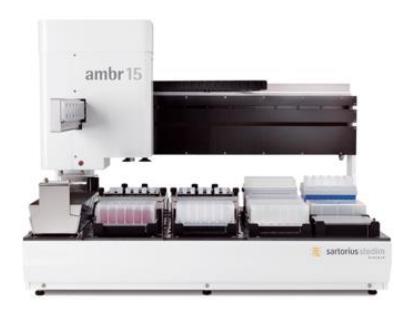

$\mathrm{C}$

Figure 5. Culture systems used in this study. (A) Shake flask, used in Chapter 2. (B) BIOSTAT STR ${ }^{\circledR} 10$ $L$ stir tank bioreactor (STR) from Sartorius, used in Chapter 3, 4, and 5. (C) ambr 15 miniature bioreactor system from Sartorius, used in Chapter 5. Figure B and C are adopted from Sartorius with their permission to be published in this thesis.

This study is part of the project consortium Animal Cell Cultivation and Screening System (ACCESS) with an aim to develop smart cultivation and screening technologies for accelerating early process development. The consortium comprises of Wageningen University, Synthon Biopharmaceuticals BV, Bioceros Holding BV, Biostream International BV, and IBIS Technologies BV with close collaboration among partners.

In Chapter 2, a study on $\mathrm{CHO}$ cell fed-batch culture platform selection is presented. The study aims to select a suitable $\mathrm{CHO}$ cell clone and a media system for the next process development and scale-up/down studies. Two $\mathrm{CHO}^{\mathrm{BC} \otimes}$ clone candidates derived from the same parent and producing the same $\mathrm{mAb}$ are used. Four basal media and three feed media are tested in fed-batch cultures in all possible combinations ( $=12$ conditions) for each clone, using shake flasks. Metabolic and physiological phenotypes associated with the individual $\mathrm{CHO}$ cell clone in the 
different media are studied. Moreover, the balancing properties of each basal medium and feed medium combination are investigated for both clones. Finally, based on the maximum biomass concentration and product titer, the BC-P clone in combination with ActiCHO-P and ActiCHO feed A\&B is selected for further process development and scale up/down in this study.

In Chapter 3 and 4, the designed fed-batch process is run in a $10 \mathrm{~L}$ bioreactor system to characterize and to better understand the dynamics of cell metabolism and physiology. These studies focus on the observed substantial cell size increase (SI) phase which occurs right after the exponential cell number increase (NI) phase. In Chapter 3, Biomass composition and metabolic flux balance analysis are performed for both phases, in order to characterize the metabolic changes during the fed-batch culture and to better understand its relation to cell size. In Chapter 4, changes in gene expression are studied upon the switch to increasing cell size to better understand regulatory mechanisms behind the cell size increase and the relation to the process conditions. Chapter 3 and 4 together result in a thorough characterization of the physiology of the $\mathrm{CHO}$ cells during the fed-batch process at the $10 \mathrm{~L}$ scale and form a sound basis for comparison to a scaled-down process in Chapter 5 .

In Chapter 5, a scale-down process for the $10 \mathrm{~L}$ scale is carried out in the $\mathrm{ambr}^{\circledR} 15$ system which contains 48 miniature bioreactors with a maximum working volume of $15 \mathrm{~mL}$. In this chapter, two different scale-down criteria are compared in the ambr ${ }^{\circledR}$ 15 system. One is based on matching both $\mathrm{O}_{2}$ transfer rate and agitation tip-speed (Vtip) with the $10 \mathrm{~L}$ process, the other is based on matching $\mathrm{O}_{2}$ transfer rate and agitation power input per volume of liquid $(\mathrm{P} / \mathrm{V})$. Finally, the scale-down process performance is evaluated and verified by comparing the $\operatorname{ambr}^{\circledR} 15$ results to the $10 \mathrm{~L}$ process in terms of cell growth, metabolism, transcriptome, mAb production, and mAb glycosylation.

This thesis investigates the $\mathrm{CHO}$ cell physiology underlying the fed-batch process dynamics. Such information is used to verify the scale-down process and can be used further to develop more representative scale-up/down systems. Finally, Chapter 6 discusses the major findings in this thesis and the current status and challenges in bioprocess scale-up/down. Additional opportunities as well as challenges can be seen by further scale-down to $\mathrm{mL}$ or even $\mu \mathrm{L}$ scales. The future perspectives are presented to better implement such systems in process development. 


\section{Chapter 2}

Selection of chemically defined media for $\mathrm{CHO}$ cell fed-batch culture processes 


\begin{abstract}
:
Two $\mathrm{CHO}$ cell clones derived from the same parental $\mathrm{CHO}^{\mathrm{BC}}{ }^{\circ}$ cell line and producing the same monoclonal antibody (BC-G, a low producing clone; BC-P, a high producing clone) were tested in four basal media in all possible combinations with three feeds ( $=12$ conditions) in fed-batch cultures. Higher amino acid feeding did not always lead to higher mAb production. The two clones showed differences in cell physiology, metabolism and optimal medium-feed combinations. During the phase transitions of all cultures, cell metabolism showed a shift represented by lower specific consumption and production rates, except for the specific glucose consumption rate in cultures fed by Actifeed A/B. The BC-P clone fed by Actifeed A/B showed a 3 -fold cell volume increase and an increase of the specific consumption rate of glucose in the stationary phase. Since feeding was based on glucose this resulted in accumulation of amino acids for this feed, while this did not occur for the poorer feed (EFA/B). The same feed also led to an increase of cell size for the BC-G clone, but to a lesser extent.
\end{abstract}

Xiao Pan ${ }^{a}$

Mathieu Streefland a

Ciska Dalm ${ }^{\mathrm{b}}$

René H. Wijffels a, c

Dirk E. Martens ${ }^{a}$

a Bioprocess Engineering, Wageningen University, PO Box 16, 6700 AA, Wageningen, the Netherlands b Synthon Biopharmaceuticals, Upstream Process Development, PO Box 7071, 6503 GN, Nijmegen, the Netherlands cFaculty of Biosciences and Aquaculture, Nord University, N-8049, Bodø, Norway

Cytotechnology 69:39-56 


\subsection{Introduction:}

Chinese Hamster Ovary $(\mathrm{CHO})$ cells are the main production host for recombinant therapeutic protein $\mathbf{s}^{13}$. Fed-batch culture is currently the most common industrial process for $\mathrm{CHO}$ cell culture. For a fed-batch process, volumetric productivity and product titer are important outputs. These outputs are a function of viable cell density $(\mathrm{VCD})$, specific productivity $\left(\mathrm{q}_{\mathrm{p}}\right)$, and culture longevity, which in turn are influenced by the medium and feed composition. Selecting a suitable culture medium and feed platform is therefore pivotal for developing a fed-batch manufacturing process. Chemically defined basal media and feeds are currently the standard for $\mathrm{CHO}$ cell fed-batch cultures. In a fed-batch culture, $\mathrm{CHO}$ cells are inoculated and start to grow in a basal medium. A feed is added to the culture when a certain culture state is reached, such as when a certain cell density is reached, a certain culture time point is reached, or a certain nutrient concentration is reached. The basal medium and especially the feed should contain a balanced set of essential nutrients in a ratio that meets the demand of the cells for proliferation and production of the pharmaceutical protein. A feed is generally more concentrated than a basal medium to maximize culture volumetric productivity and product titer. Studies have shown that the composition of culture media and feeds can influence cell growth, protein productivity ${ }^{44}$, gene expression ${ }^{45}$, product quality ${ }^{10,46,47}$, and metabolism of lactate and ammonia ${ }^{6,45,48,49}$.

To design a chemically defined medium in-house is time and labor consuming. Currently, process development of $\mathrm{CHO}$ cell culture usually starts with commercially available culture media systems as a basis ${ }^{44}$. Several well performing chemically defined media have become commercially available for $\mathrm{CHO}$ cells. These media are designed based on different strategies to reach high volumetric productivities and product titers. For instance, some media are designed to boost cell growth to a high cell density, others are designed to extend the longevity of cells or to enhance cell specific productivity. For different medium-feed combinations the richness and composition of the basal media and feeds differ widely. The optimal composition of a basal medium or a feed is highly dependent upon the basic type of $\mathrm{CHO}$ cell used, specific characteristics of the generated sub-clones, and type of product ${ }^{44,47}$. Thus, there is no single basal medium or feed suitable for all $\mathrm{CHO}$ cultures.

A problem with media selection is that different $\mathrm{CHO}$ cell types and clones have different nutrient requirements and therefore for each clone candidate the medium and feed formulation and feeding strategies would have to be screened, designed and optimized. Generally this information is not disclosed by manufacturers, which makes the early process development difficult for start-ups. Some studies were conducted and reported to select or compare commercial media for $\mathrm{CHO}$ cell cultures. Rodrigues et al. ${ }^{44}$ have compared viable cell concentration and antibody production in seven commercially available media in batch cultures of a CHO-K1 cell 
line and stressed the importance of selecting the correct medium for the outcome of a process. Reinhart et al. ${ }^{47}$ compared cell growth, antibody production, antibody quality on eight chemically defined media for a CHO DG44 cell line in batch, and with a subset of conditions in fed-batch.

To investigate how differences between clones derived from the same parental cell line may affect the choice of basal media and feeds in fed batch processes. This study investigates the effects of different basal media and feeds on cell growth, metabolism, physiology, and $\mathrm{mAb}$ production for two differentially behaving clones derived from the same parental cell line. The two clones were compared in 12 basal medium-feed combinations, resulting in total 24 fed-batch culture conditions. Each clone was best supported by a different basal medium-feed combination, even though they are derived from the same parental cell line. Clear metabolic shifts are observed for all the cultures when going from the growth phase to the stationary phase. Also the type and extent of these metabolic shifts are different for the 2 clones for the different basal medium-feed combinations used. Cultures fed by Actifeed A/B showed a marked increase in cell volume, which also was different for both clones being respectively 3-fold for BC-P and 2-fold for BC-G. Further improvements of cell growth and volumetric productivity by tailoring feed composition and/or feeding strategy based on specific cellular requirement are discussed.

\subsection{Materials and methods}

\subsubsection{Cell line and culture medium}

Two Chinese Hamster Ovary $(\mathrm{CHO}) \mathrm{BC}^{\circledR}$ (Provided by Bioceros Holding BV, The Netherlands) suspension cell clones with different growth and production patterns being BC-G (high maximum cell density, low productivity) and BC-P (low maximum cell density, high productivity) were used. Both clones were derived from the same parental cell line by the same transfection and cell line generation program, and produce the same recombinant human monoclonal antibody (mAb), immunoglobulin G1 (IgG1). Each clone contained two constructs: one with a heavy chain and one with a light chain, where each construct had a different resistance marker allowing for a double selection.

Four chemically defined basal media were compared being: 1. CD FortiCHO ${ }^{\text {TM }}$ (Forti, or FortiCHO) Medium, 2. CD OptiCHO ${ }^{\mathrm{TM}}$ (Opti, or OptiCHO) Medium, 3. CD-CHO Medium, and 4. ActiCHO-P (Acti, or ActiCHO) medium. With 1, 2 and 3 purchased from Life Technologies and 4 purchased from GE Healthcare. In combination with these basal media, three chemically defined feed systems were compared being: 1 . Efficient Feed $^{\mathrm{TM}}$ A and B (EFA/B) from Life Technologies added in a ratio of 1:1 (v/v), based on supplier's information; 2. Efficient Feed ${ }^{\text {TM }}$ C (EFC) from Life Technologies; 
and 3. ActiCHO Feed ${ }^{\mathrm{TM}}-\mathrm{A}$ and $-\mathrm{B}$ (Acti A/B, or Actifeed A/B) from GE Healthcare added in a ratio 10:1 (v/v), based on supplier's information. All basal media were supplemented with $4 \mathrm{mM}$ L-glutamine (Gibcoß, Life Technologies) and $0.5 \%$ (v/v) Anti-clumping agent (Gibco ${ }^{\circledR}$, Life Technologies). All possible combinations of these media and feeds $(3 \times 4)$ were compared for the two $\mathrm{CHO}^{\mathrm{BC} \otimes}$ clones in fed-batch cultures.

\subsubsection{Pre-culture and fed-batch culture}

One ampoule of cells from a working cell bank was thawed in FortiCHO medium in a $125 \mathrm{~mL}$ un-baffled shake flask (VWR, USA) with a $12 \mathrm{~mL}$ working volume. Next, the thawed culture was washed with fresh FortiCHO medium and sub-cultured in the four different basal media supplemented with selection reagents $(200 \mu \mathrm{g} / \mathrm{mL}$ Zeocin ${ }^{\mathrm{TM}}$ and $5 \mu \mathrm{g} / \mathrm{mL}$ blasticidin, both from Life technologies). Cells were subcultured every 3 days (1 passage) in the exponential growth phase to an initial density of $2 \times 10^{5}$ cells $/ \mathrm{mL}$ for at least five passages and considered adapted when they had a stable specific growth rate $(\mu)$ for two consecutive passages. Just before inoculation of the fed-batch cultures, cells were spun down at $300 \times \mathrm{g}$. Spent medium was discarded and cells were re-suspended in fresh medium without selection reagents. The actual fed-batch culture experiments were done in $250 \mathrm{~mL}$ shake flasks (VWR, USA) with a $25 \mathrm{~mL}$ initial working volume in duplicate at a seeding density of $2 \times 10^{5}$ cells $/ \mathrm{mL}$. Cultures were incubated with $100 \mathrm{rpm}$ shaking speed and $50 \mathrm{~mm}$ orbital shaking diameter in an incubator (Multitron $\mathrm{CO}_{2}$ incubator; Infors HT) operated with $8 \% \mathrm{CO}_{2}$ at $37{ }^{\circ} \mathrm{C}$ and $90 \%$ humidity. Feed addition started from culture day 3 on for all the tested conditions with a daily bolus addition. The determination of feed supply rate is explained in section 2.2.6. Cultures were harvested on day 14 , or when viability dropped below $60 \%$.

\subsubsection{Cell density and viability determination}

Samples were taken daily starting from culture day 2. A $1 \mathrm{~mL}$ sample was taken from each culture flask for measurements of total cell density, viable cell density and cell diameter using an Automated Cell Counter (TC20 ${ }^{\mathrm{TM}}$; BIO-RAD) by trypan blue dye (Sigma-Aldrich) exclusion method. Next, the sample was filtered through a $0.2 \mu \mathrm{m}$ filter (Minisart, Sartorius ${ }^{\mathrm{TM}}$ ). The cell free flow-through was stored at $-20{ }^{\circ} \mathrm{C}$ for later analysis.

\subsubsection{Culture spent medium, osmolality and product titer analysis}

Glucose and lactate concentrations were daily measured using an YSI analyzer (YSI 2700, YSI Life Sciences). Ammonium concentrations were analyzed by a BioProfile FLEX analyzer (Nova biomedical) after the experiment was finished. Spent medium composition including all the extracellular amino acids was quantified using NMR 
(Spinnovation biologics BV) based on the sample scheme (Table I) after the experiment was finished. IgG concentration was determined by Bioceros Netherlands BV, using the Octet system with protein G biosensors (ForteBio). Osmolality was measured using an Osmomat 030-D cryoscopic osmometer (Gonotec).

Table I. Sample points for spent media analysis. Samples for spent media analysis were carried out on every other day for each culture. Grey cells: culture was terminated.

\begin{tabular}{ccccccccccc}
\hline & Day & $\mathbf{0}$ & $\mathbf{2}$ & $\mathbf{4}$ & $\mathbf{6}$ & $\mathbf{8}$ & $\mathbf{1 0}$ & $\mathbf{1 2}$ & $\mathbf{1 3}$ & $\mathbf{1 4}$ \\
\hline \multirow{5}{*}{ BC-P } & Opti + EFA/B & $\sqrt{ }$ & $\sqrt{ }$ & $\sqrt{ }$ & $\sqrt{ }$ & $\sqrt{ }$ & $\sqrt{ }$ & & & \\
& Opti + Acti A/B & $\sqrt{ }$ & $\sqrt{ }$ & $\sqrt{ }$ & $\sqrt{ }$ & $\sqrt{ }$ & $\sqrt{ }$ & $\sqrt{ }$ & & $\sqrt{ }$ \\
& Acti + EFA/B & $\sqrt{ }$ & $\sqrt{ }$ & $\sqrt{ }$ & $\sqrt{ }$ & $\sqrt{ }$ & $\sqrt{ }$ & $\sqrt{ }$ & $\sqrt{ }$ & \\
& Acti + Acti A/B & $\sqrt{ }$ & $\sqrt{ }$ & $\sqrt{ }$ & $\sqrt{ }$ & $\sqrt{ }$ & $\sqrt{ }$ & $\sqrt{ }$ & & $\sqrt{ }$ \\
\hline \multirow{5}{*}{ BC-G } & Opti + EFA/B & $\sqrt{ }$ & $\sqrt{ }$ & $\sqrt{ }$ & $\sqrt{ }$ & $\sqrt{ }$ & $\sqrt{ }$ & $\sqrt{ }$ & & $\sqrt{ }$ \\
& Opti + Acti A/B & $\sqrt{ }$ & $\sqrt{ }$ & $\sqrt{ }$ & $\sqrt{ }$ & $\sqrt{ }$ & $\sqrt{ }$ & $\sqrt{ }$ & & \\
& Acti + EFA/B & $\sqrt{ }$ & $\sqrt{ }$ & $\sqrt{ }$ & $\sqrt{ }$ & $\sqrt{ }$ & $\sqrt{ }$ & $\sqrt{ }$ & & $\sqrt{ }$ \\
& Acti + Acti A/B & $\sqrt{ }$ & $\sqrt{ }$ & $\sqrt{ }$ & $\sqrt{ }$ & $\sqrt{ }$ & $\sqrt{ }$ & $\sqrt{ }$ & $\sqrt{ }$ \\
\hline
\end{tabular}

\subsubsection{Productivity}

Based on cell growth patterns of fed-batch cultures, four culture phases were distinguished:

1. Start-up phase (L-Phase), from the inoculation day until day 2;

2. Exponential growth phase (G-phase), from day 2 until the day that the cultures reached the maximum cell density;

3. Stationary phase (S-Phase), from the end of G-Phase until the culture viability was lower than $80 \%$; and

4. Death phase (D-Phase), is-from the end of S-Phase until the end of the culture.

This study mainly focuses on the G- and the S-phase during the cultures. The calculation of average specific consumption/production rates was done separately for the growth and stationary phase (Figure 8).

Change in the total amount of compound $x$ in the shaker flask is given by:

$$
\frac{d M_{x}}{d t}=q_{x} \cdot M_{V C}(t) \quad \text { Equation } 1
$$

Where $\mathrm{Mx}_{\mathrm{x}}(\mathrm{mg}$; mmol) is the total amount of compound $\mathrm{x}$ in a culture, Mvc (cells) is the number of viable cells in a culture and $q_{x}\left(\mathrm{mmol} \cdot \mathrm{cell}^{-1} \cdot \mathrm{day}^{-1}\right)$ is the specific production rate of compound x. With constant $\mathrm{q}_{\mathrm{x}}$, integration of Equation 1 gives:

$$
M_{x}(t)-M_{x}(0)=q_{x} \cdot \int_{0}^{t} M_{V C} \quad \text { Equation } 2
$$

The average specific production rates of glucose, essential amino acids and antibody were obtained from a plot of the total amount of a compound against the integral of 
viable cell numbers using linear regression. Positive values indicate production while negative values indicate consumption.

\subsubsection{Feed supply}

Glucose serves as one of the main carbon and energy sources for $\mathrm{CHO}$ cells ${ }^{50}$. The consumption rate of glucose is often used as an indicator of cell culture activity to guide feeding strategies. Since the specific consumption rate of glucose at per cell level does not vary much if cell activity is unchanged and glucose concentrations are well above the Monod saturation constant. In this study, it was not possible to conduct daily measurement for nutrients other than glucose and lactate. The daily amount of feed addition, therefore, was based on the glucose concentration at the moment of feeding and the anticipated glucose consumption rate for the next 24 hours according to glucose specific consumption rate of the cells. The feeding strategy used in this study is different from the supplier's protocol. However, since the protocols differ among suppliers, the feeding strategy of this study gives fairer comparison between different feed systems. The feeding strategy presumes that the glucose concentration in the feed is well balanced with the rest of the nutrients. To calculate the daily feed amount, the following method was used. First, the specific growth rate $\mu_{8}\left(\right.$ day $\left.^{-1}\right)$ is calculated by

$$
\mu_{g}=\frac{\ln \frac{V C D_{Y}}{V C D_{Y-1}}}{t_{Y}-t_{Y-1}} \quad \text { Equation } 3
$$

Where VCDY-1 and VCDY (cells $\cdot \mathrm{mL}^{-1}$ ) are the viable cell densities of the last and the current feeding points, respectively. $t_{Y-1}$ and $t_{Y}$ (day) are the time points for the last and the current feeding points, respectively. Then, the VCD of the next day can be predicted based on Equation 4.

$$
V C D_{Y+1}=V C D_{Y} \times e^{\mu_{g} \cdot\left(t_{Y+1}-t_{Y}\right)} \quad \text { Equation } 4
$$

Next the integral viable cell density (IVCD) of the previous and the next culture day was calculated by Equation 5 and Equation 6 respectively.

$$
\begin{array}{ll}
\Delta I V C D_{Y}=V C D_{Y-1} \times\left(t_{Y}-t_{Y-1}\right)+\frac{\left(V C D_{Y}-V C D_{Y-1}\right) \times\left(t_{Y}-t_{Y-1}\right)}{2} & \text { Equation } 5 \\
\Delta I V C D_{Y+1}=V C D_{Y} \times\left(t_{Y+1}-t_{Y}\right)+\frac{\left(V C D_{Y+1}-V C D_{Y}\right) \times\left(t_{Y+1}-t_{Y}\right)}{2} & \text { Equation } 6
\end{array}
$$

IVCD was defined as the area under the viable cell growth curve using the trapezium rule. $\triangle \mathrm{IVCD}$ y and $\triangle \mathrm{IVCDY}+1\left(\right.$ cells $\cdot$ day $\left.\cdot \mathrm{mL}^{-1}\right)$ are the IVCD of the past day and the predicted next day respectively.

The specific glucose consumption rate $\left(q_{\mathrm{Gluc}}, \mathrm{mmol} \cdot \mathrm{cell}^{-1} \cdot \mathrm{day}^{-1}\right)$ can then be calculated by: 


$$
\frac{\Delta G L C_{Y}}{\Delta I V C D_{Y}}=q_{G l c_{Y}} \text { Equation } 7
$$

Assuming the specific glucose consumption rate stays constant, the glucose consumption during the next day $\left(\triangle \mathrm{GLC}_{\mathrm{Y}+1}, \mathrm{mM}\right)$ can then be predicted by:

$$
\Delta G L C_{Y+1}=q_{G c_{Y}} \times \Delta I V C D_{Y+1} \quad \text { Equation } 8
$$

Finally, the feed volume to be added was determined by using Equation 9. Glucose concentration was kept at $10 \mathrm{mM}$ (as a safe threshold) by the next day sample point.

$$
V_{F}=\frac{\left(\Delta G L C_{Y+1}+\left(10-G L C_{Y}\right)\right) \times V_{Y}}{C_{F}} \quad \text { Equation } 9
$$

Where GLCY is the current glucose concentration $(\mathrm{mM}), \mathrm{V}_{\mathrm{F}}$ is the feed volume to be added $(\mathrm{mL}), \mathrm{VY}_{\mathrm{Y}}$ is the current culture volume $(\mathrm{mL})$ and $\mathrm{CF}_{\mathrm{F}}$ is the glucose concentration in the feed $(\mathrm{mM})$.

For each duplicate culture the feeding volume was calculated. Next the two obtained values were averaged and the average feeding volume was added to both cultures. By using this feeding method, we managed to keep the range of glucose between 10 to $30 \mathrm{mM}$ throughout the fed-batch cultures by daily bolus feeding.

\subsubsection{Balance of essential amino acids in feeds}

To check whether the essential amino acid and glucose concentrations in the feed are balanced with respect to the needs of the cells, a relative balancing level is defined

$$
\text { Relative balancing level }=\left(\frac{\frac{c_{E A A_{X}}}{C_{g l c}}}{\frac{q_{E A A_{x}}}{q_{g l c}}}\right) \text { Equation } 10
$$

Where $C_{E A A x}$ and $C_{g l c}($ all in $\mathrm{mM}$ ) are concentrations of essential amino acid $\mathrm{x}$ and glucose in the feed, $q_{E A A x}$ and $q_{g l c}\left(\right.$ all in $\mathrm{mmol} \cdot \mathrm{cell}^{-1}$. day $^{-1}$ ) are the specific consumption rates of essential amino acid $x$ and glucose during the growth phase or the stationary phase of the culture. In this way, the ratio of essential amino acid $x$ to glucose is compared between the formulation of feed and the cell specific consumption rates. Since the feed supply rate was based on glucose concentration in this experiment, the feed balance of essential amino acids was evaluated based on glucose. A relative balancing level equal to 1 means that the rate with which the essential amino acid is supplied by the feed equals its consumption rate when feed addition is based on glucose. A value other than 1 means that the supply rate of the essential amino acid is either lower $(<1)$ than or higher $(>1)$ than the specific consumption rate, resulting in either depletion or accumulation. 


\subsection{Results}

\subsubsection{Media and feed compositions}

The composition of the used basal media and feeds was measured with respect to amino acids and glucose (Figure 1). All basal media and feeds contain glucose, nonessential amino acids and essential amino acids. In general, the feeds are 5 to 10 times more concentrated than the basal media in both glucose and amino acids. For the basal media, OptiCHO is relatively poorer whereas FortiCHO and ActiCHO-P are richer in amino acids content. For the feeds, Actifeed a/b is richer than EFA/B (3-fold) and EFC (2-fold) in amino acids and glucose content.
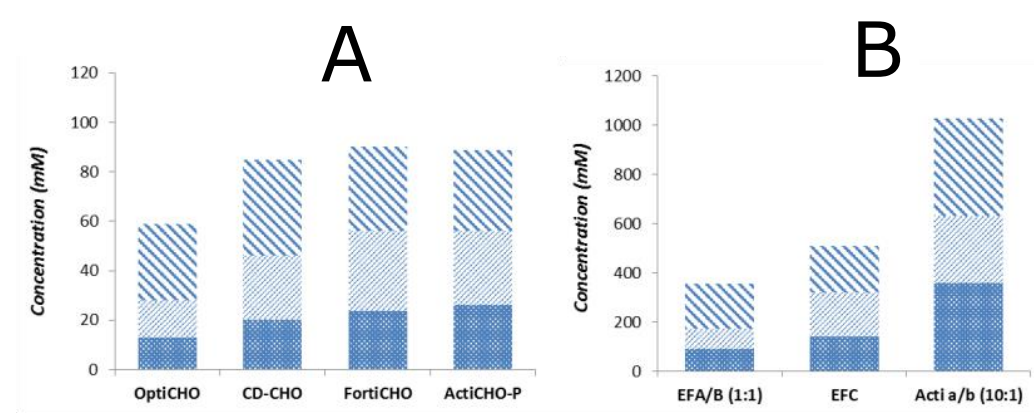

NGlucose $/ / /$ Non-essential amino acids Essential amino acids

Figure 1. Sum of concentrations of non-essential amino acids, of essential amino acids, and concentration of glucose in different basal media (A) and feeds $(B)$. EFA and EFB are used in a ratio of 1:1 (v/v) and Actifeed $a / b$ in a ratio of 10:1 (v/v).

\subsubsection{Performance of BC-P and BC-G clones in different medium-feed combinations}

Volumetric productivity and product titer of the mAb are the most often used parameters during media and clone selection in the early culture process development phase. Therefore the volumetric productivity and IgG product titer of all the studied conditions were compared (Figure 2). For the BC-G clone, FortiCHO + $\mathrm{EFA} / \mathrm{B}$ resulted in the highest titer $\left(190 \mathrm{mg} \cdot \mathrm{L}^{-1}\right)$ and volumetric productivity $\left(14 \mathrm{mg} \cdot \mathrm{L}^{-}\right.$ ${ }^{1} \cdot$ day $\left.^{-1}\right)$. For the BC-P clone, ActiCHO + Actifeed A/B resulted in the highest titer $\left(1250 \mathrm{mg} \cdot \mathrm{L}^{-1}\right)$ and volumetric productivity $\left(90 \mathrm{mg} \cdot \mathrm{L}^{-1} \cdot \mathrm{day}^{-1}\right)$. Overall, the BC-P clone showed clearly broader ranges (up to 6-fold higher) of IgG titer and volumetric productivity compared to the $\mathrm{BC}-\mathrm{G}$ clone. Compared to the other basal media, the use of $\mathrm{CD}-\mathrm{CHO}$ resulted in the lowest volumetric productivities and product titers for both clones irrespective of the feed used. Other output parameters including maximum viable cell density, integral viable cell density, specific growth rate, number of days where the viability is above $80 \%$ and specific productivity can be found in Table II. 
Cell physiology responded differently to medium-feed combinations. Remarkably, for both clones when fed by Actifeed $\mathrm{A} / \mathrm{B}$, an increase of cell diameter during the stationary phase was observed. The increase was not observed when the other feeds (EFA/B and EFC) were used (data in Figure 3 only shows EFA/B). For the BC-P clone the size increase was most prominent with an increase of the average cell diameter from 16 to $24 \mu \mathrm{m}$ (Figure 3), which is equivalent to a cell volume increase of nearly 3fold. The cell size increase was also observed for the BC-G clone fed by Actifeed A/B, but to a lesser extent being from 14 to $18 \mu \mathrm{m}$. For the culture using Opti+Actieed A/B with the BC-G clone in Figure 3, the cell size increase could not be observed because it was terminated on day 9 due to a low viability. The observation implies a common response between the two clones towards the feed addition. The sudden decrease of cell diameter in the end of the cultures was due to cell death.
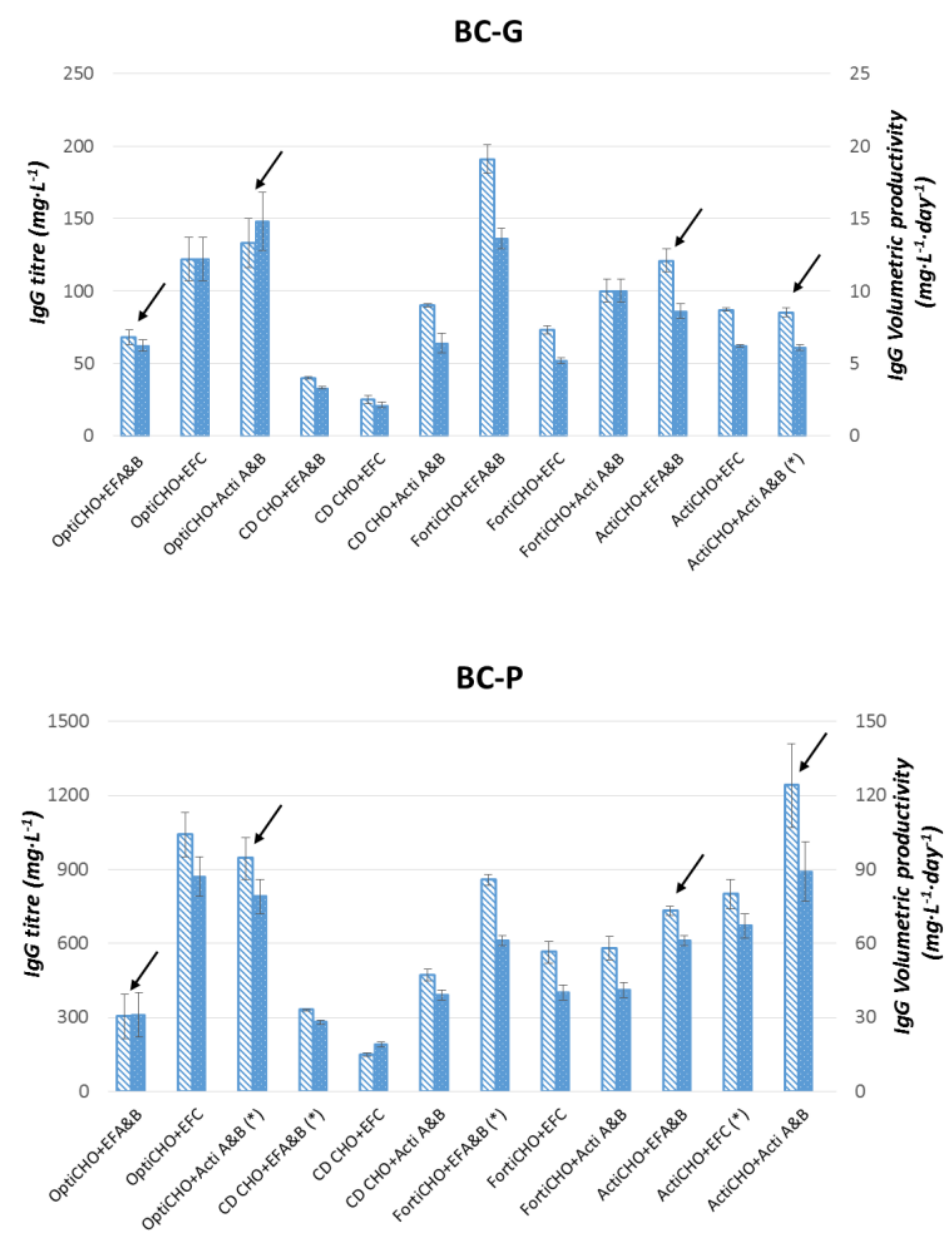

$\mathbb{Q}$ Product titre

Volumetric productivity

Figure 2. Product titer $\left(m g \cdot L^{-1}\right)$ and volumetric productivity $\left(m g \cdot L^{-1} \cdot d a y^{-1}\right)$ of clone BC-G and BC-P for all tested basal medium-feed combinations. Error bars indicate the deviation of the duplicate data points from the average value. Conditions marked with arrows were selected for further comparisons. Conditions marked with (*) indicate cell aggregation during the cultures. 
Table II. Fed-batch culture results of clone BC-G and BC-P for all possible combinations of 4 types of media and 3 feed systems. " \pm " indicates the deviation of the duplicate data points from the average value. Asterisks at the max viable cell density values indicate cell aggregate during the cultures.

Conditions marked in blue are selected for further comparison.

\begin{tabular}{|c|c|c|c|c|c|c|c|c|c|c|c|c|c|}
\hline \multirow{2}{*}{\multicolumn{2}{|c|}{ Platforms }} & \multicolumn{3}{|c|}{ OptiCHO } & \multicolumn{3}{|c|}{ CD-CHO } & \multicolumn{3}{|c|}{ FortiCHO } & \multicolumn{3}{|c|}{ ActiCHO } \\
\hline & & \multirow{2}{*}{$\begin{array}{c}\text { EFA\&B } \\
68 \\
\pm 5\end{array}$} & \multirow{2}{*}{$\begin{array}{l}\text { EFC } \\
122 \\
\pm 15\end{array}$} & \multirow{2}{*}{$\begin{array}{c}\begin{array}{c}\text { ActiFeed } \\
\text { A\&B }\end{array} \\
133 \\
\pm 17\end{array}$} & \multirow{2}{*}{$\begin{array}{c}\text { EFA\&B } \\
\mathbf{4 0} \\
\pm 1\end{array}$} & \multirow{2}{*}{$\begin{array}{c}\mathrm{EFC} \\
25 \\
\pm 3\end{array}$} & \multirow{2}{*}{$\begin{array}{c}\begin{array}{c}\text { ActiFeed } \\
\text { A\&B }\end{array} \\
90 \\
\pm 1\end{array}$} & \multirow{2}{*}{$\begin{array}{c}\text { EFA\&B } \\
191 \\
\pm 10\end{array}$} & \multirow{2}{*}{$\begin{array}{l}\text { EFC } \\
73 \\
\pm 3\end{array}$} & \multirow{2}{*}{$\begin{array}{c}\begin{array}{c}\text { ActiFeed } \\
\text { A\&B }\end{array} \\
100 \\
\pm 8\end{array}$} & \multirow{2}{*}{$\begin{array}{c}\text { EFA\&B } \\
\begin{array}{c}121 \\
\pm 8\end{array}\end{array}$} & \multirow{2}{*}{$\begin{array}{c}\text { EFC } \\
87 \\
\pm 1\end{array}$} & \multirow{2}{*}{$\begin{array}{c}\begin{array}{c}\text { ActiFeed } \\
\text { A\&B }\end{array} \\
85 \\
\pm 3\end{array}$} \\
\hline \multirow{7}{*}{ BC-G } & $\begin{array}{l}\text { Product titre } \\
\left(\mathrm{mg} \cdot \mathrm{L}^{-1}\right)\end{array}$ & & & & & & & & & & & & \\
\hline & $\begin{array}{l}\text { Volumetric } \\
\text { productivity } \\
\left(\mathrm{mg}^{-1} \cdot \mathrm{L}^{-1} \cdot \text { day }^{-1}\right)\end{array}$ & $\begin{array}{c}6.2 \\
+0.4\end{array}$ & $\begin{array}{r}12.2 \\
+1.5\end{array}$ & $\begin{array}{l}14.8 \\
+2.0\end{array}$ & $\begin{array}{c}3.3 \\
\pm 0.1\end{array}$ & $\begin{array}{c}2.1 \\
\pm 0.2\end{array}$ & $\begin{array}{l}6.4 \\
\pm 0.7\end{array}$ & $\begin{array}{l}13.6 \\
\pm 0.7\end{array}$ & $\begin{array}{l}5.2 \\
\pm 0.2\end{array}$ & $\begin{array}{l}10.0 \\
\pm 0.8\end{array}$ & $\begin{array}{c}8.6 \\
+0.5\end{array}$ & $\begin{array}{c}6.2 \\
+0.1\end{array}$ & $\begin{array}{c}6.1 \\
+0.2\end{array}$ \\
\hline & $\begin{array}{c}\text { Max. viable } \\
\text { density } \\
\left(\cdot 10^{6} \mathrm{~mL}^{-1}\right)\end{array}$ & $\begin{array}{c}12 \\
+0.3\end{array}$ & $\begin{array}{c}\mathbf{1 1} \\
\pm 0.7\end{array}$ & $\begin{array}{c}15 \\
\pm 0.2\end{array}$ & $\begin{array}{c}\mathbf{9} \\
\pm 0.1\end{array}$ & $\begin{array}{l}7.8 \\
\pm 0.6\end{array}$ & $\begin{array}{l}8.2 \\
\pm 0.7\end{array}$ & $\begin{array}{c}19 \\
+1.7\end{array}$ & $\begin{array}{c}13 \\
\pm 0.6\end{array}$ & $\begin{array}{c}11 \\
\pm 0.3\end{array}$ & $\begin{array}{c}12 \\
\pm 0.7\end{array}$ & $\begin{array}{c}9^{*} \\
\pm 0.2\end{array}$ & $\begin{array}{c}11 \\
\pm 0.5\end{array}$ \\
\hline & $\begin{array}{c}\text { Specific } \\
\text { Growth rate } \\
\text { (day }^{-1} \text { ) }\end{array}$ & 0.71 & 0.67 & 0.72 & 0.65 & 0.62 & 0.65 & 0.72 & 0.71 & 0.71 & 0.55 & 0.56 & 0.55 \\
\hline & $\begin{array}{l}\text { High-viability } \\
\text { days (day) }\end{array}$ & 8 & 8 & 7 & $8-9$ & 9 & 8 & 14 & 11 & $7-8$ & 11 & $10-11$ & 10 \\
\hline & $\begin{array}{l}\text { Max specific } \\
\text { productivity } \\
\left.\text { (pg-cell } \text { I.1.day }^{-1}\right)\end{array}$ & $\begin{array}{c}1.2 \\
\pm 0.09\end{array}$ & $\begin{array}{c}2.1 \\
\pm 0.02\end{array}$ & $\begin{array}{c}2.1 \\
\pm 0.1\end{array}$ & $\begin{array}{c}0.6 \\
\pm 0.03\end{array}$ & $\begin{array}{c}0.6 \\
\pm 0.005\end{array}$ & $\begin{array}{c}0.6 \\
\pm 0.026\end{array}$ & $\begin{array}{c}1.4 \\
\pm 0.01\end{array}$ & $\begin{array}{c}1.0 \\
\pm 0.005\end{array}$ & $\begin{array}{l}1.3 \\
\pm 0.7\end{array}$ & $\begin{array}{c}1.5 \\
\pm 0.1\end{array}$ & $\begin{array}{c}1.3 \\
\pm 0.22\end{array}$ & $\begin{array}{c}1.5 \\
\pm 0.12\end{array}$ \\
\hline & $\begin{array}{c}\text { Integral viable } \\
\text { cell density } \\
\text { (IVCD) } \\
\left(\cdot 10^{7} \cdot \text { day }^{\prime} \cdot \mathrm{ml}^{1}\right)\end{array}$ & 8 & 6.3 & 6.5 & 5.6 & 4.7 & 5.9 & 12 & 9 & 5.4 & 8.8 & 6 & 7.5 \\
\hline \multirow{7}{*}{ BC-P } & $\begin{array}{l}\text { Product titre } \\
\left(\mathrm{mg} \cdot \mathrm{L}^{-1}\right)\end{array}$ & $\begin{array}{l}305 \\
\pm 90\end{array}$ & $\begin{array}{c}1041 \\
\pm 91\end{array}$ & $\begin{array}{l}945 \\
\pm 85\end{array}$ & $\begin{array}{c}330 \\
\pm 4\end{array}$ & $\begin{array}{c}148 \\
\pm 7\end{array}$ & $\begin{array}{l}471 \\
\pm 23\end{array}$ & $\begin{array}{l}857 \\
\pm 22\end{array}$ & $\begin{array}{l}564 \\
\pm 45\end{array}$ & $\begin{array}{l}580 \\
\pm 48\end{array}$ & $\begin{array}{l}731 \\
\pm 19\end{array}$ & $\begin{array}{l}800 \\
\pm 60\end{array}$ & $\begin{array}{l}1240 \\
\pm 170\end{array}$ \\
\hline & $\begin{array}{l}\text { Volumetric } \\
\text { productivity } \\
\left(\mathrm{mg}^{-1} \cdot \mathrm{L}^{-1} \cdot \mathrm{day}^{-1}\right)\end{array}$ & $\begin{array}{l}31 \\
\pm 9\end{array}$ & $\begin{array}{l}87 \\
\pm 8\end{array}$ & $\begin{array}{l}79 \\
\pm 7\end{array}$ & $\begin{array}{l}28 \\
\pm 1\end{array}$ & $\begin{array}{l}19 \\
\pm 1\end{array}$ & $\begin{array}{l}39 \\
\pm 2\end{array}$ & $\begin{array}{l}61 \\
\pm 2\end{array}$ & $\begin{array}{l}40 \\
\pm 3\end{array}$ & $\begin{array}{l}41 \\
\pm 3\end{array}$ & $\begin{array}{l}61 \\
\pm 2\end{array}$ & $\begin{array}{l}67 \\
\pm 5\end{array}$ & $\begin{array}{r}89 \\
\pm 12\end{array}$ \\
\hline & $\begin{array}{c}\text { Max. viable } \\
\text { density } \\
\left(-10^{6} \mathrm{~mL}^{-1}\right)\end{array}$ & $\begin{array}{l}10.5 \\
\pm 0.6\end{array}$ & $\begin{array}{c}\mathbf{1 0} \\
\pm 0.2\end{array}$ & $\begin{array}{l}13 * \\
\pm 0.8\end{array}$ & $\begin{array}{l}5.5^{*} \\
\pm 0.4\end{array}$ & $\begin{array}{l}3.2 \\
\pm 0.6\end{array}$ & $\begin{array}{l}4.6 \\
\pm 0.4\end{array}$ & $\begin{array}{l}10^{*} \\
\pm 0.6\end{array}$ & $\begin{array}{l}7.5 \\
\pm 0.4\end{array}$ & $\begin{array}{c}9.6 \\
\pm 0.8\end{array}$ & $\begin{array}{l}7.6 \\
\pm 0.1\end{array}$ & $\begin{array}{c}7^{*} \\
\pm 0.1\end{array}$ & $\begin{array}{c}11 \\
\pm 0.4\end{array}$ \\
\hline & $\begin{array}{c}\text { Specific } \\
\text { Growth rate } \\
\text { (day }{ }^{11} \text { ) }\end{array}$ & 0.65 & 0.63 & 0.68 & 0.46 & 0.43 & 0.48 & 0.65 & 0.60 & 0.63 & 0.61 & 0.59 & 0.67 \\
\hline & $\begin{array}{l}\text { High-viability } \\
\text { days (day) }\end{array}$ & 7 & 9 & 10 & 3-4 & $3-4$ & 6 & 13 & 11 & 7 & 7 & 9 & 12 \\
\hline & $\begin{array}{c}\text { Max specific } \\
\text { productivity } \\
\left.\text { (pg.cell } \text { I.1.day }^{-1}\right)\end{array}$ & $\begin{array}{l}6.9 \\
\pm 0.9\end{array}$ & $\begin{array}{l}15.8 \\
\pm 0.8\end{array}$ & $\begin{array}{l}\mathbf{1 2 . 3} \\
\pm 0.7\end{array}$ & $\begin{array}{l}10.5 \\
\pm 0.7\end{array}$ & $\begin{array}{l}\mathbf{1 0 . 6} \\
\pm 0.1\end{array}$ & $\begin{array}{l}13.9 \\
\pm 0.4\end{array}$ & $\begin{array}{l}11.3 \\
\pm 0.4\end{array}$ & $\begin{array}{l}9.9 \\
\pm 0.5\end{array}$ & $\begin{array}{c}8.2 \\
\pm 0.2\end{array}$ & $\begin{array}{l}13.6 \\
\pm 0.3\end{array}$ & $\begin{array}{l}\mathbf{1 3 . 2} \\
\pm 1.5\end{array}$ & $\begin{array}{l}16.7 \\
\pm 0.8\end{array}$ \\
\hline & $\begin{array}{l}\text { Integral viable } \\
\text { cell density } \\
\text { (IVCD) } \\
\left(\cdot 10^{7} \cdot \text { day }\left.^{-1}\right|^{-1}\right)\end{array}$ & 5.3 & 8.1 & 9.3 & 3.2 & 2 & 3.7 & 7.4 & 5.6 & 6.9 & 5.4 & 4.6 & 7.7 \\
\hline
\end{tabular}




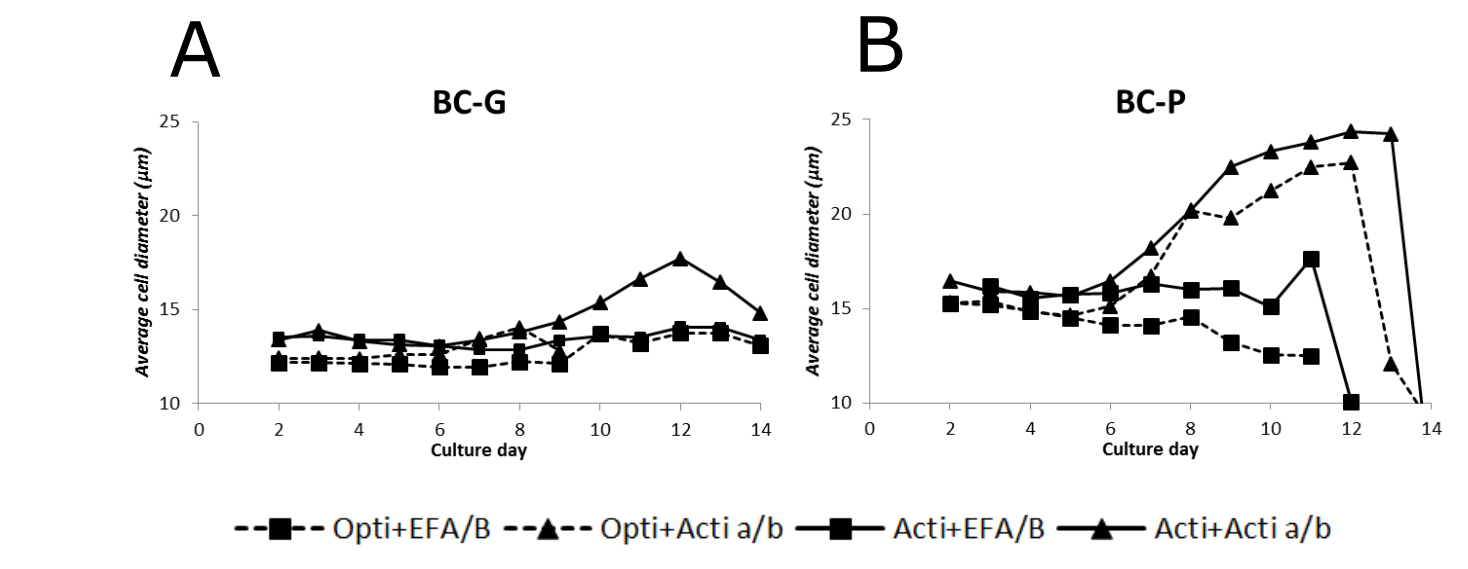

Figure 3. Average cell diameters ( $\mu \mathrm{m})$ of the BC-G (A) and BC-P (B) clones in the selected fed-batch culture conditions.

\subsubsection{Metabolite profiles in fed-batch cultures}

To study the effect of different medium-feed combinations on cell metabolism, 4 combinations (marked with arrows in Figure 2) were selected for further comparison being: a relatively poor medium $(\mathrm{OptiCHO})$ with a poor feed $(\mathrm{EFA} / \mathrm{B})$ and a rich feed (Actifeed $\mathrm{A} / \mathrm{B}$ ) and a rich basal medium (ActiCHO) with the same feeds. In this way, the impact of the richness of media and feeds was compared in more detail. Amongst these conditions the cultures in which the highest volumetric productivities were obtained (BC-G in OptiCHO + Actifeed A/B and BC-P in ActiCHO + Actifeed A/B) are included. For these conditions spent media analysis was conducted on different time points according to Table I.

Figure 4 shows the viable cell density and viability of the selected culture conditions. For the BC-G clone, the growth patterns are similar when using the same basal medium, while for the BC-P clone, the growth patterns are similar when using the same feed. In all medium-feed combinations, cell division stopped after culture day 6 , except for the $\mathrm{BC}-\mathrm{G}$ clone in ActiCHO medium, where cell division lasted until day 8 but at a lower maximum specific rate of $\sim 0.55$ /day compared to $\sim 0.70$ /day for the BC-G clone in OptiCHO medium (Table II). The decline of viable cell density during the stationary phase was caused by dilution due to sampling and feeding, and/or due to cell death. 

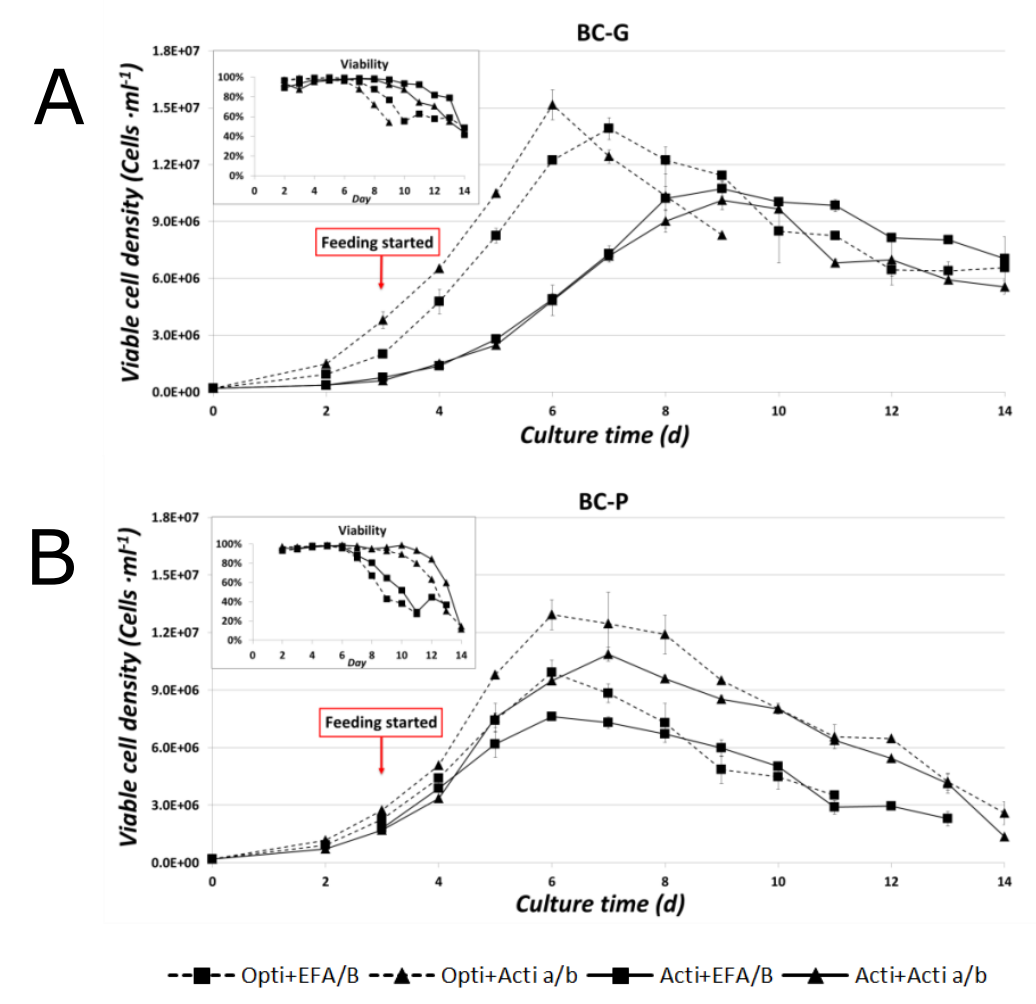

Figure 4. Viable cell density and viability of the BC-G (A) and BC-P (B) clones in the selected conditions. Error bars show the deviation of the duplicate data points from the average value.

\subsubsection{Glucose, lactate, ammonium and osmolality profiles}

Glucose shows similar zigzag profiles for the tested conditions due to consumption and daily feed addition starting from day 3 on (Figure 5 A\&E). For the BC-P clone fed by Actifeed A/B during the stationary phase, a considerably higher daily glucose consumption is observed (Figure $5 \mathbf{E}$ ). For both clones the lactate concentration shows a similar trend with an initial increase followed by a decrease after 3 to 4 days except again for the BC-P clone fed by Actifeed A/B, where the lactate concentration kept increasing until the end of the culture. The observed simultaneous consumption of lactate and glucose agrees with literature on various other $\mathrm{CHO}$ cell lines ${ }^{15,45,51}$. Ammonium levels keep increasing during all fed-batch cultures. For the BC-P clone fed with Actifeed $A / B$, clearly higher ammonium concentrations are observed (Figure $5 \mathrm{C} \& \mathrm{G}$ ). The osmolality is constant except for the cultures fed by Actifeed $\mathrm{A} / \mathrm{B}$, where an increase up to 400 and $620 \mathrm{mOsm} / \mathrm{kg}$ is observed for respectively the $\mathrm{BC}-\mathrm{G}$ and the BC-P clone (Figure $5 \mathbf{D \& H}$ ). Both clones seem to cope well with the osmolality since the viability maintained higher than $80 \%$ until day 12 . 

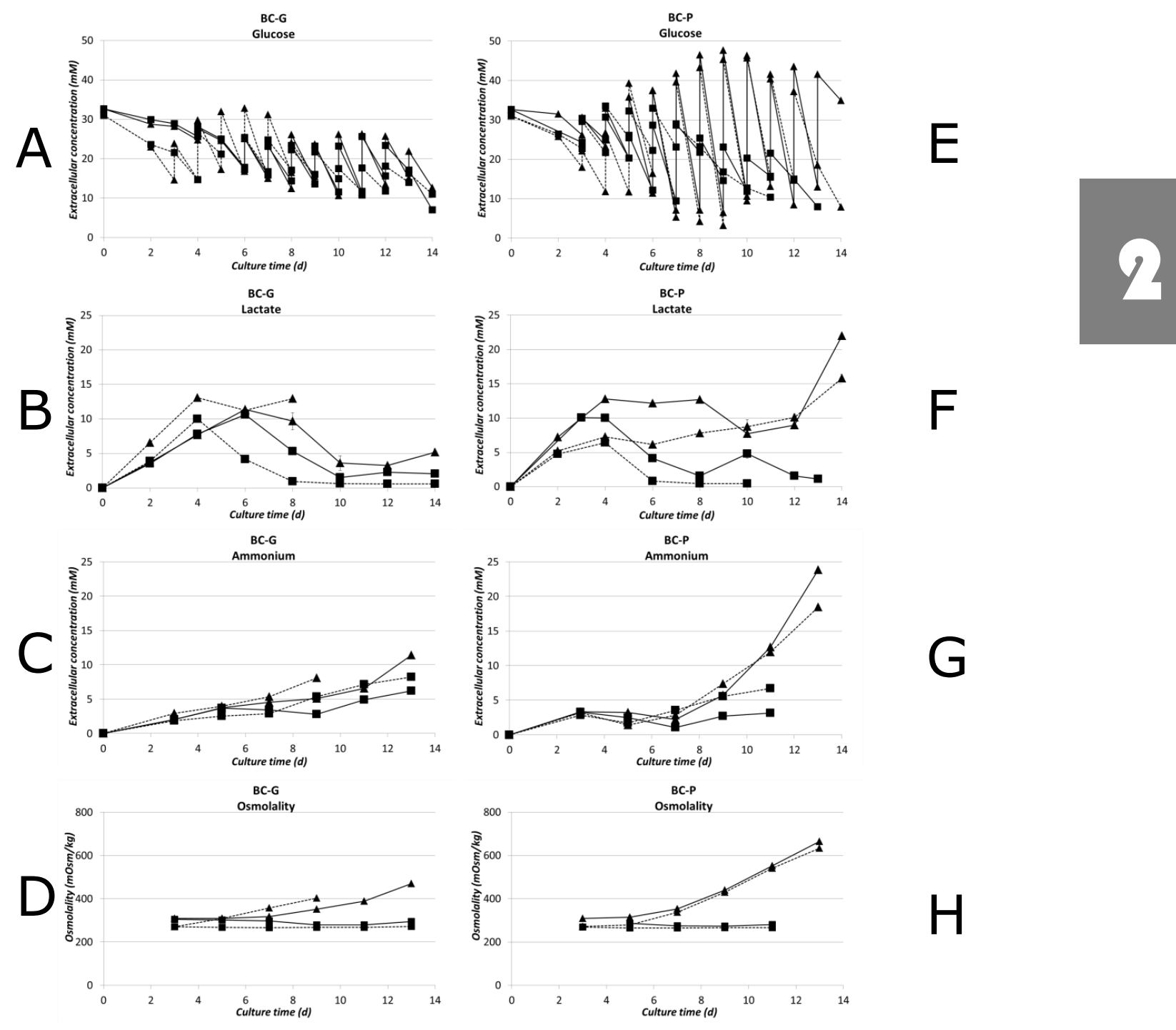

--口-- Opti+EFA/B --A-- Opti+Acti a/b $\longrightarrow$ - Acti+EFA/B $\longrightarrow$ - Acti+Acti a/b

Figure 5. Extracellular concentration of glucose, lactate, ammonium (Graph A, B, C, E, F and G: unit in $\mathrm{mM}$ ) and culture osmolality (Graph D and $\mathrm{H}$ : unit in $\mathrm{mOsm} / \mathrm{kg}$ ) for the two clones during fed-batch cultures. Error bars show the deviation of the duplicate data points from the average value.

\subsubsection{Essential amino acid profiles}

In each culture condition, the essential amino acid profiles show similar trends. As an example, threonine concentrations of the selected culture conditions are shown (Figure 6). None of the essential amino acids was depleted during fed-batch cultures, indicating that they were not the limiting nutrients for cell growth or production in all studied cases. Cultures fed by EFA/B resulted in stable essential amino acid concentrations, whereas cultures fed by Actifeed A/B resulted in accumulation of essential amino acids starting from the stationary phase (day 6). The accumulation was larger for the BC-P clone than for the BC-G clone. 


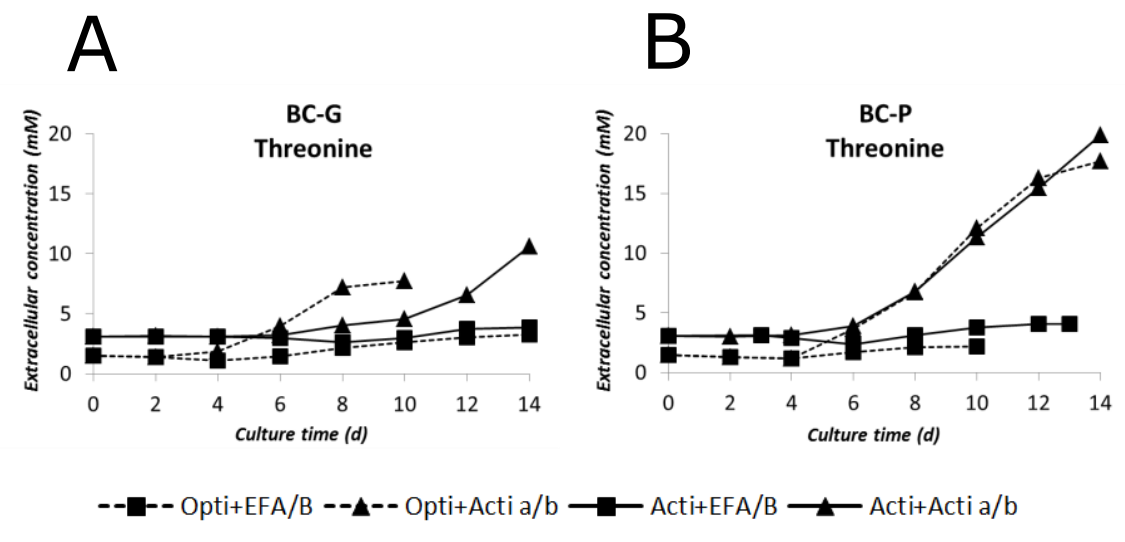

Figure 6. Extracellular threonine concentrations $(m M)$ during fed-batch cultures of the both clones. Error bars show the deviation of the duplicate data points from the average value.

\subsubsection{Non-essential amino acid profiles}

Figure 7 shows the profiles of asparagine, aspartic acid, glutamine, glutamic acid and alanine, which are the non-essential amino acids directly related to the primary carbon metabolism. In most of the conditions, asparagine and glutamine were depleted between day 4 and day 6 (Figure 7 A, C, F and H). When using Actifeed $\mathrm{A} / \mathrm{B}$ feed, glutamine, which is not present in the feed, was produced in the later culture stage as indicated by the increased glutamine concentration. The sudden increase of asparagine concentration at day 14 in Figure 7 F was most likely a measurement error, since it was not observed throughout other cultures. Aspartic acid and glutamic acid stayed at low concentrations during EFA/B fed cultures, but increased during Actifeed A/B fed cultures (Figure 7 B, D, G and I), because of the high content of these two compounds ( $\sim 50 \mathrm{mM}$ and $\sim 70 \mathrm{mM}$ respectively) in Actifeed $\mathrm{A} / \mathrm{B}$. Alanine is not present in the basal media, but is present in EFA/B feed $(\sim 1 \mathrm{mM})$ and Actifeed A/B $(\sim 16 \mathrm{mM})$. As seen in Figure $7 \mathrm{E} \& \mathrm{~J}$, all selected culture conditions showed an increase of alanine concentration except for the poor medium and poor feed $($ OptiCHO $+\mathrm{EFA} / \mathrm{B})$ where alanine was consumed after day 6. In the cultures with the rich medium and poor feed $(\mathrm{ActiCHO}+\mathrm{EFA} / \mathrm{B})$, the alanine increase was mostly due to production by cells, whereas when fed by the rich feed (Actifeed A/B) the increase was due to both feeding and production. When fed by feed EFA/B, depletions of tyrosine and cystine were observed at around day 6 for both the clones (see Appendix 4 of the online published data). 

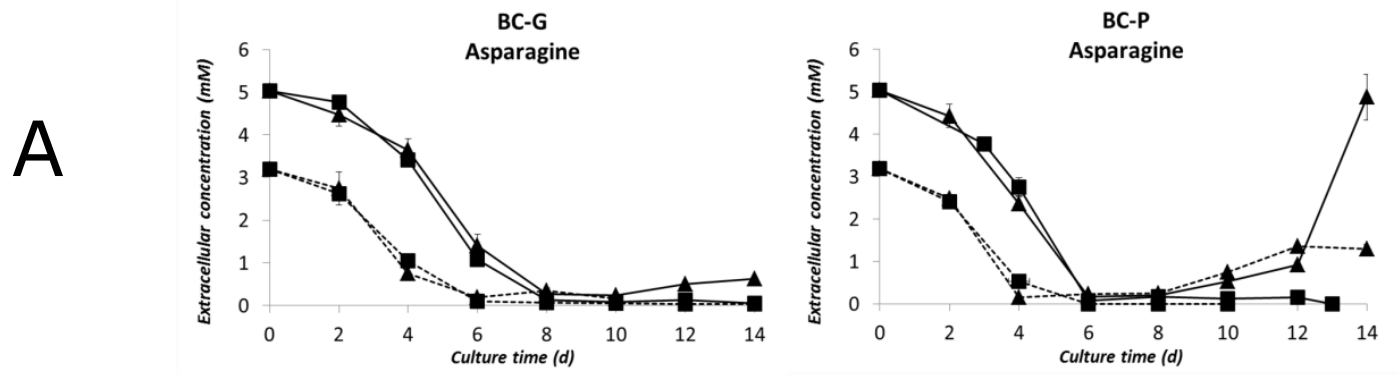

BC-G
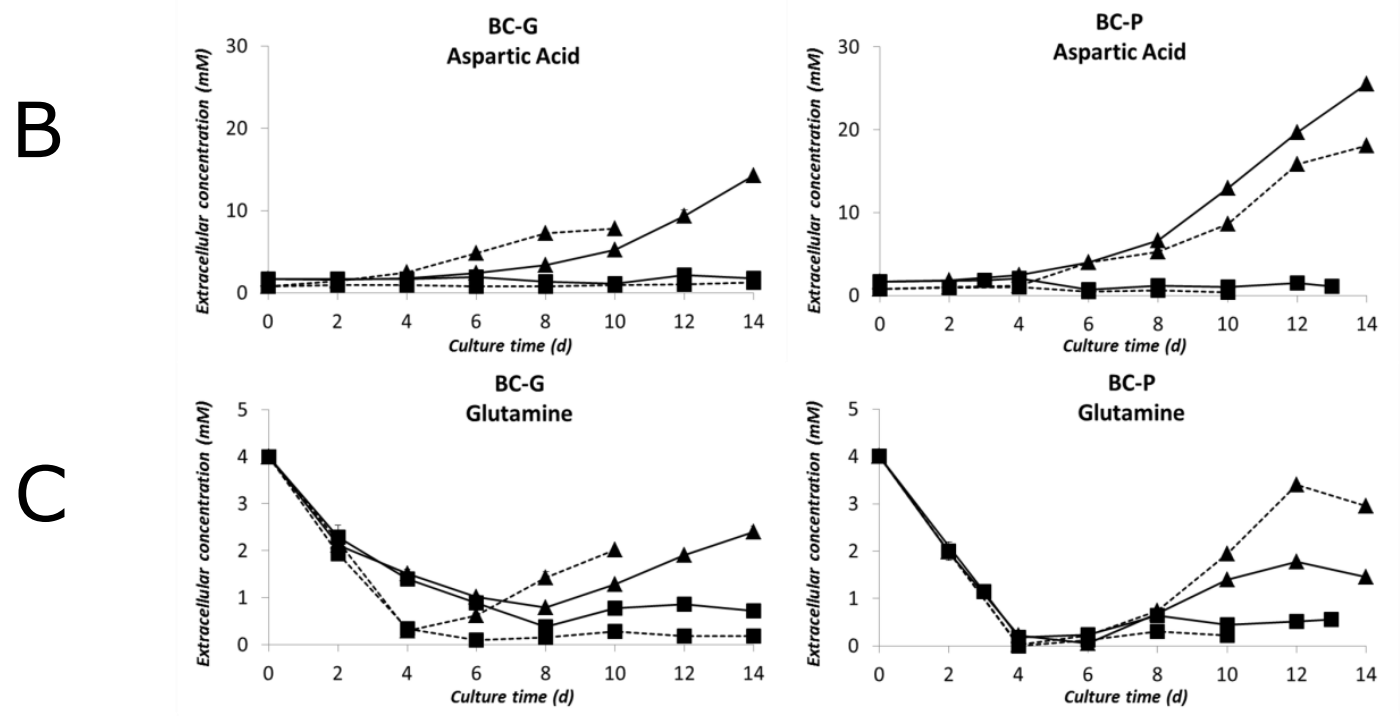

$G$
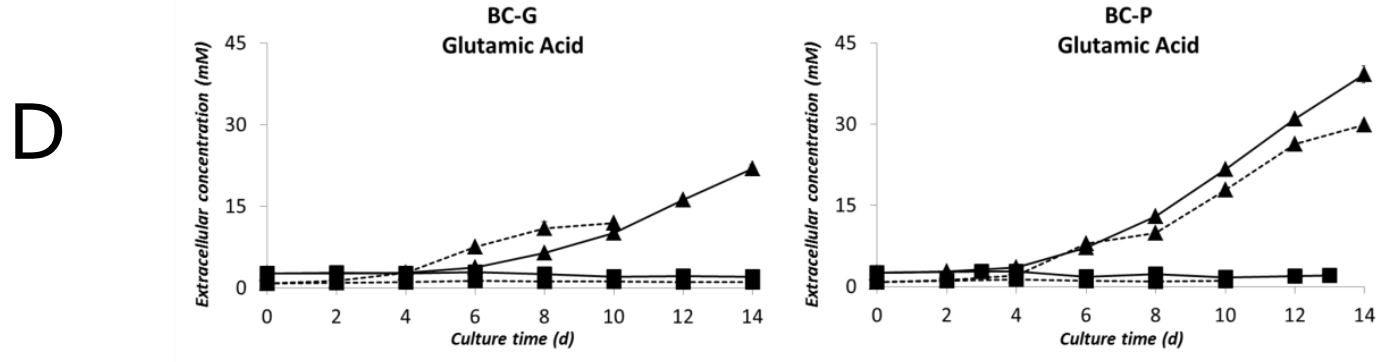

$H$
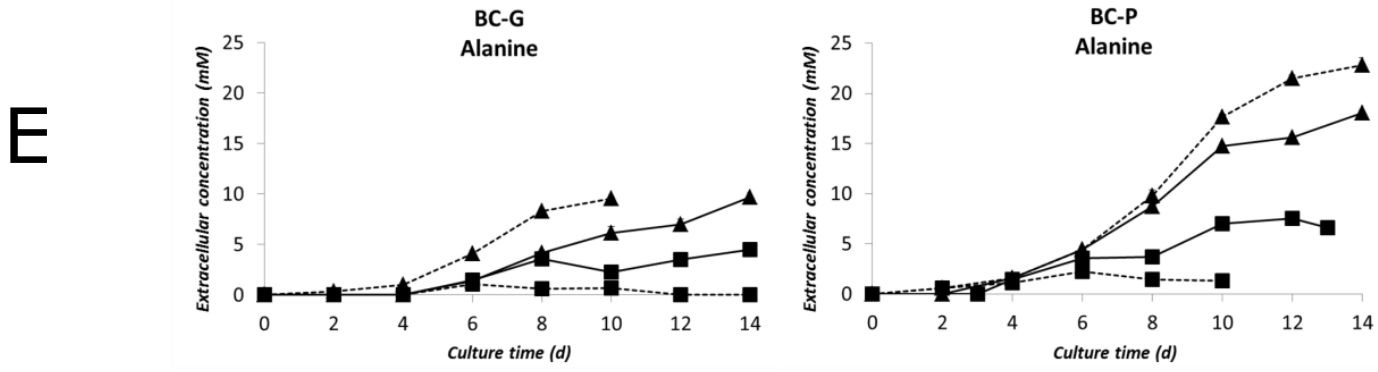

I

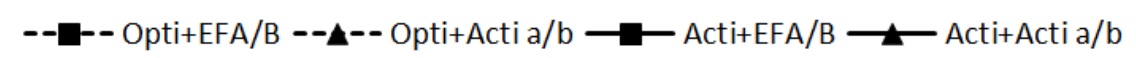

Figure 7. Non-essential amino acid profiles of selected culture conditions in fed-batch cultures. Concentrations are shown in $m M$. Graph $A, B, C, D, E$ show the profiles of asparagine, aspartic acid, glutamine, glutamic acid and alanine of the BC-G clone; Graph $F, G, H, I, J$ show their profiles of the $B C-P$ clone. Error bars show the deviation of the duplicate data points from the average value. 


\subsubsection{Specific consumption rates}

In a fed-batch culture, the essential amino acid feeding rate should match the consumption rates of the cells. The average specific consumption rates of essential amino acids and glucose are presented during the growth and the stationary phase for both clones (Figure 8). Note that on average, the ratios among essential amino acids for two clones are similar during the growth phase, indicating similar requirements for both clones to make biomass during this phase. In general, average specific consumption rates of most of the essential amino acids during the growth phase are significantly ( $\mathrm{T}$ tests, data not shown) higher than during the stationary phase. This is also true for the average specific consumption rate of glucose, except for the BC-P clone fed by Actifeed A/B, where the average specific glucose consumption rate is higher in the stationary phase than in growth phase (Figure 8 C\&D). Overall, the BC-P clone shows higher average specific glucose and essential amino acids consumption rates compared to the BC-G clone, which may be related to the larger cell size of the BC-P clone $(\sim 16 \mu \mathrm{m})$ compared to the BC-G clone $(\sim 13 \mu \mathrm{m})$ (Figure 3). In order to show the variation of specific glucose consumption rate during, a boxplot of the specific glucose consumption rate for all the tested conditions during different culture phases is shown in Figure 9. The specific glucose consumption rate varies in a wide range in different media/feed conditions for the BC-P clone, whereas in a much smaller range for the BC-G clone. 


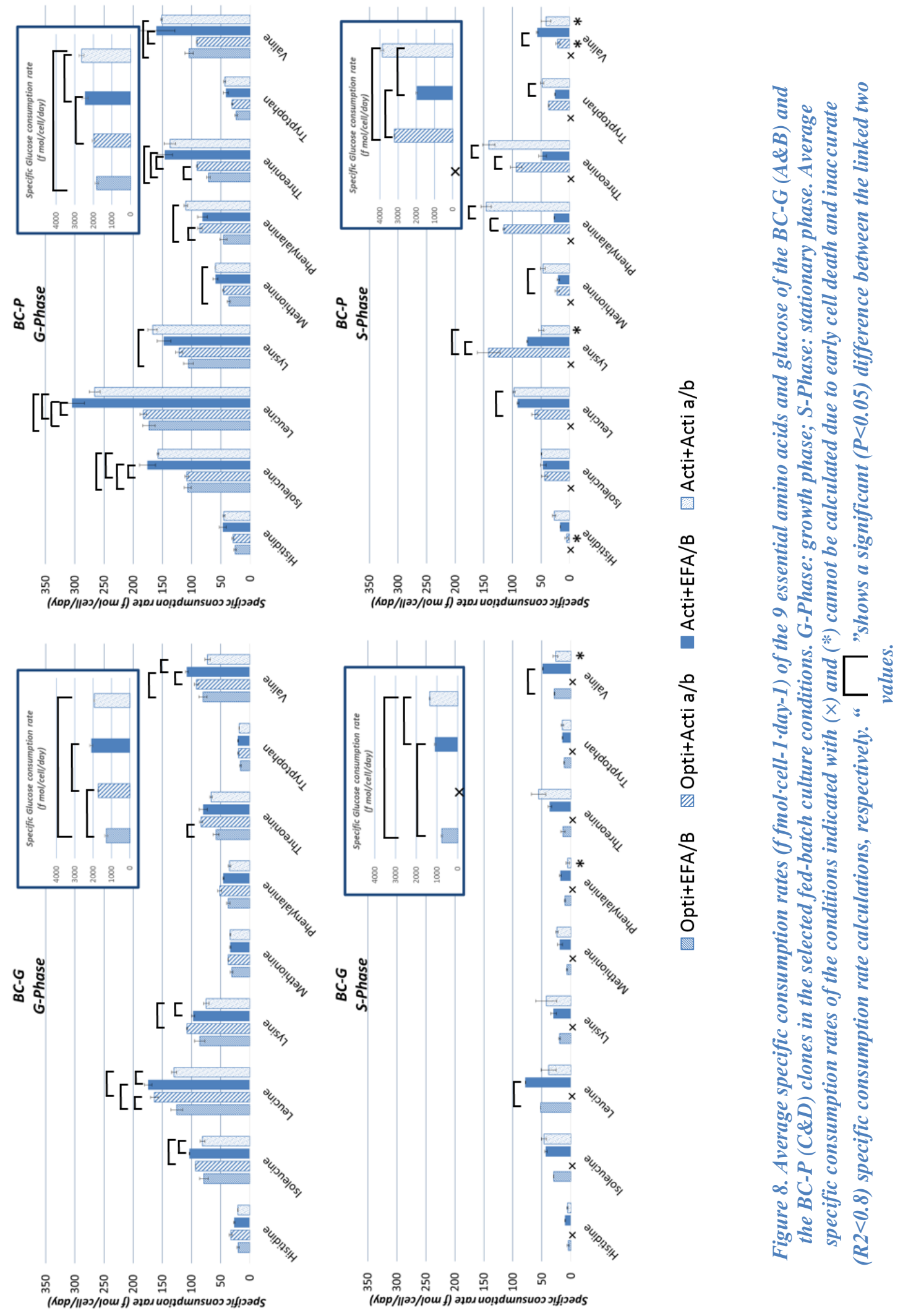




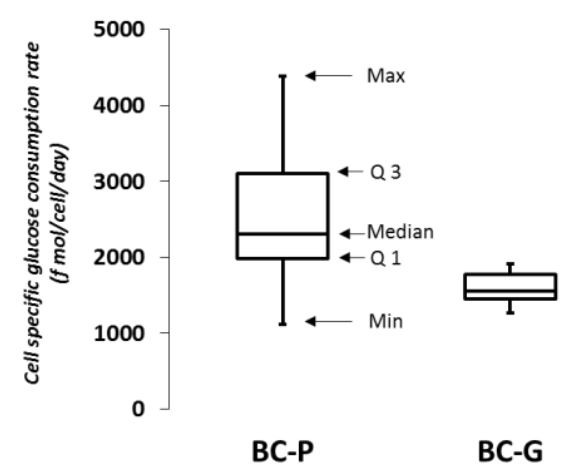

Figure 9. Overall cell specific glucose consumption rates ( $\mathrm{fmol} / \mathrm{cell} / \mathrm{day})$ for the BC-P and BC-G clone ranges observed in all the tested conditions. The maximum (Max), minimum (Min), median, quartile 1 (Q1), and quartile 2 (Q2) values are shown by arrows for the BC-P clones; same follows for the BC-G clone.

\subsection{Discussion}

\subsubsection{Effects of media and feed on culture performances and cell physiology}

In total, 12 combinations of four basal media and three feeds were tested for two $\mathrm{CHO}$ daughter clones. As expected, the BC-P clone reached higher volumetric productivities and product titers. Overall, no clear correlation between richness level of the media or feed and the performance of the clones was observed. For example, the BC-G clone reached the highest titer $\left(191 \mathrm{mg} \cdot \mathrm{L}^{-1}\right)$ in FortiCHO $+\mathrm{EFA} / \mathrm{B}$, which is a rich medium with a poor feed, while for this clone ActiCHO with Actifeed A/B, which is a rich medium with a rich feed performed clearly poorer (Figure 2). For both clones, $\mathrm{CD}-\mathrm{CHO}$ medium did not support mAb production. Notably, even though the 2 clones are generated from the same parent and produce the same $\mathrm{mAb}$, they do not share a common medium-feed for their optimal mAb production.

It was observed that the addition of Actifeed A/B resulted in a cell volume increase for both the clones with a 3-fold increase for the BC-P clone and a 2-fold increase for the BC-G clone. The increase of cell size did not occur when fed by EFA/B and EFC, which indicates that the cell size increase is not an intrinsic property of the cell line, but rather a physiological response to the feed system. Several possibilities might cause the size increase: 1 . Cell cycle arrest. It was reported that overexpression of a cell cycle inhibitor ( $\mathrm{p}^{21^{\mathrm{CIP} 1}}$ ) in a $\mathrm{CHO}$ cell line resulted in a 4 -fold increase of cell volume $^{52}$ for overexpression of another inhibitor (p27) in a $\mathrm{CHO}$ cell line a 2-fold increase of cell protein content was measured ${ }^{53}$. When cells are arrested at the $\mathrm{G}_{1}$ phase, they continue to grow in size instead of passing the dividing checkpoint. It might be that in this study the depletion of certain nutrients has led to a cell cycle arrest. Note that there are still many nutrients (eg. vitamins and trace elements) in the basal media and feeds that were not measured in this study. 2. Another possibility 
could be that the overfed nutrients resulted in higher rate of cellular transportation of amino acids, the accumulation of these precursors triggers the higher formation rate of biopolymers. Oh et al. ${ }^{54}$ demonstrated an increase of amino acid uptake via $\mathrm{Na}^{+}-$ dependent transport systems caused by elevation of osmolality. 3. It might also be that the cells get larger by taking up water. However, this is typically seen when cells are exposed to hypotonic pressure ${ }^{55}$. Furthermore, it was observed that a higher average $\mathrm{mAb}$ specific productivity is correlated with conditions that result in a larger cell size (the BC-P clone fed by Actifeed A/B; Table II). Cell specific mAb productivity has been reported to correlate with cell volume $52,56,57$. Kim et al.58 suggested a linear correlation between the increase in specific thrombopoietin productivity and the increase of cell size for the $9 \mathrm{CHO}$ sub-clones they studied. Results from this study seem to agree with their observation. However, this experiment is unable to present the precise comparison of the specific mAb productivity before and after the cell size increase, since the cultures were performed in uncontrolled shake flasks with culture volumes constantly changing by sampling and feeding.

The BC-P clone tended to form aggregates in more medium-feed combinations compared to the BC-G clone (indicated by the asterisks in Figure 2). Formation of aggregates indicates suboptimal growth conditions as aggregates were reported to be formed around decaying and dead cells through the released DNA ${ }^{59}$. In this study, addition of $0.5 \%$ anti-clumping agent in the basal media helped to alleviate the aggregating problem, especially to the BC-P clone. The longevity of cultures, therefore, was prolonged up to 4 more days (data not shown).

\subsubsection{Cell metabolism and feed composition}

Around day 4-6 all cultures showed a transition from the growth phase to the stationary phase, which is commonly observed for $\mathrm{CHO}$ cells in batch and fed-batch cultures ${ }^{51,60-62}$. Nevertheless, the relation between overall metabolite profiles and this transition is still poorly understood, which is due to the highly complex and dynamic nature of $\mathrm{CHO}$ cell metabolism and the complexity of the media used. In this section, metabolite profiles are described with a focus on the changes that occurred during the culture phase transition. It is clear from section 2.3.3.1 that the transition from growth to stationary phase was not caused by depletion of glucose or essential amino acids. It is also not likely that the transition was caused by lactate and ammonium inhibition, since the concentrations of lactate and ammonium at the transition points were below the reported growth inhibition levels of $5-8 \mathrm{mM}$ for ammonia ${ }^{63,64}$ and 30 $\mathrm{mM}$ for lactate ${ }^{65}$. In most of the tested conditions, however, glutamine and asparagine were depleted between day 4 and day 6 (Figure 7), which coincided with the phase transition, and a shift of lactate metabolism from net production to net consumption. For the BC-G clone cultured in ActiCHO medium, depletion of asparagine and glutamine (Figure 7) occurred later probably as a consequence of a 
lower growth rate (Table II). For this culture the transition to stationary phase and the shift in lactate metabolism were also delayed (Figure 5B). It was reported that the depletion of asparagine and glutamine coincides with $\mathrm{CHO}$ cell growth suppression ${ }^{66}$. On the other hand, as can be seen in this study, glutamate and glutamine were accumulated at the later stage of the cultures. Since glutamine is not present in the feed, this shows that the $\mathrm{CHO}$ cells used in this study can produce glutamine from glutamate and therefore glutamine is not likely limiting the cultures. Asparagine is present in the feed. Although asparagine was depleted during the later stage of the cultures, it might be converted to aspartate by cells resulting in the accumulation of aspartate (Figure 7 B\&G). In the combination of OptiCHO + EFA/B, apart from asparagine and glutamine, also tyrosine and cystine were depleted around the culture transition point for both clones, which might be related to the lower mAb productivities (Table II). Similar observations of tyrosine and cystine depletion affecting protein production were shown for $\mathrm{CHO}$ cells ${ }^{67}$ and hybridoma cells ${ }^{68}$. The non-essential amino acids can be produced by cells, therefore the effects of their depletion at extracellular level to the culture performance could not be confirmed in this study. The essential amino acids however, cannot be made by cells. It is important to avoid their depletion.

Specific consumption rates of glucose and amino acids are expected to decrease when a culture shifts from the growth to the stationary phase ${ }^{60}$ due to decrease of the specific growth rate. However, in contrast to all the other tested conditions, for the BC-P clone fed by Actifeed A/B, the average specific glucose consumption rate

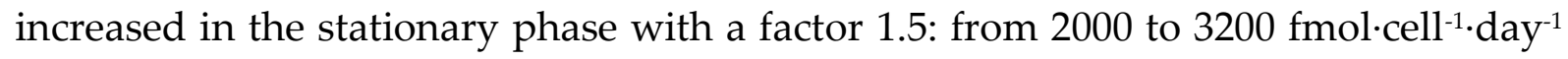

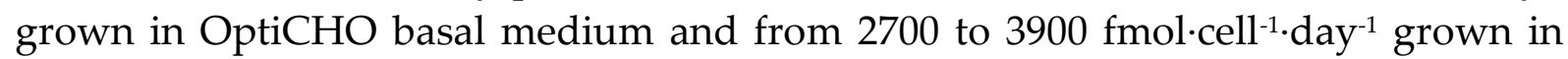
ActiCHO basal medium (Figure 8). The higher specific glucose consumption rate correlates with fact that cells become bigger (only for BC-G in Opti + Actifeed A/B this is not observed, because this culture had to be stopped before the increase became apparent). It is likely that bigger cells need more energy for growth and maintenance which is supplied by higher glucose consumption rates. It is also worth noting that when the average glucose consumption rate is calculated per cell volume (data not shown), a drop is observed upon the transition from growth to stationary phase just as with the other conditions. For the BC-P clone also more lactate is formed during the stationary phase (Figure 5 F), which means even more glucose needs to be consumed, since lactate formation is less efficient for ATP generation. In addition, production of alanine for both clones was observed (Figure 7 E\&J). The production was not observed when both clones were cultured in poor medium-feed combinations (OptiCHO + EFA/B). Extra carbons generated from glycolysis can also be diverted to alanine production through transaminase. This step at the same time transports amine groups out of the cells. Richer media and feeds (eg. ActiCHO + Actifeed A/B) supply sufficient amino acid nitrogen to make all the needed alanine and thus alanine was even produced in these cases, while for the poor media and 
feeds (eg. OptiCHO + EFA/B) this is not the case and additional alanine is taken up by the cells.

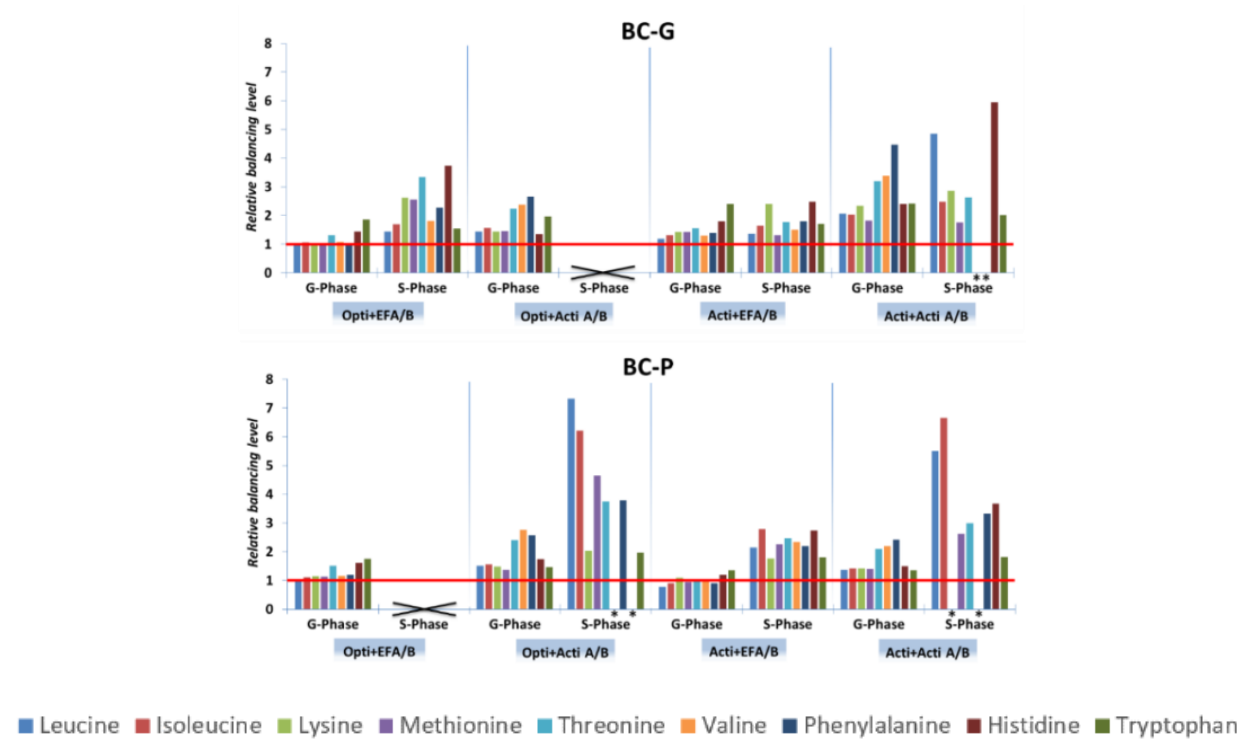

Figure 10. Relative feed balancing level of essential amino acids in different culture conditions during growth phase (G-Phase) and stationary phase (S-Phase). A value other than 1 means that the supply of the essential amino acid is either lower $(<1)$ than or higher $(>1)$ than the specific consumption rate, resulting in either depletion or accumulation. Relative balancing levels indicated with $(\times)$ and (*) cannot be calculated due to early cell death and inaccurate $\left(R^{2}<0.8\right)$ specific consumption rate calculations, respectively.

Correlated with the drastic increase of cell size for the BC-P clone fed by Actifeed $\mathrm{A} / \mathrm{B}$, glucose metabolic activity showed large variation for this clone (Figure 9). Since the feeding strategy in this experiment was based on glucose consumption, the addition of other nutrients may be out of balance. In order to evaluate the balance of the feeds, we studied the relative balancing levels of the feeds for each condition (Figure 10). The definition of the relative balancing level can be found in the material and methods section (2.7). For the growth phase of both clones, both feeds are reasonably well balanced. Only for the combination of the BC-G clone with the rich medium and feed (ActiCHO with Actifeed A/B) balancing of the feed Actifeed A/B is poorer with concentrations of essential amino acids being too high relative to the glucose concentration. Upon the transition of growth to stationary phase the specific consumption rates of amino acids changes in a different way than the specific glucose consumption rate (Figure 8), which results in an increase of balancing level values for most of the amino acids, and explains the overfeeding for these amino acids (Figure 6). Especially for the BC-P clone fed by Actifeed A/B feed, accumulation of essential amino acids occurred when the feed addition is based on glucose consumption, due to the fact that the specific consumption rate of glucose increased while the specific consumption rates of amino acids decreased during the stationary phase. Increased amino acid levels could result in increased catabolic breakdown, which explains the increase in ammonium levels. High ammonia and glutamic acid 
levels would also favor formation of alanine through the transaminase reaction ${ }^{63,69}$ and formation of glutamine through glutamine synthase, which explains the formation of these compounds in these cultures (Figure 11).

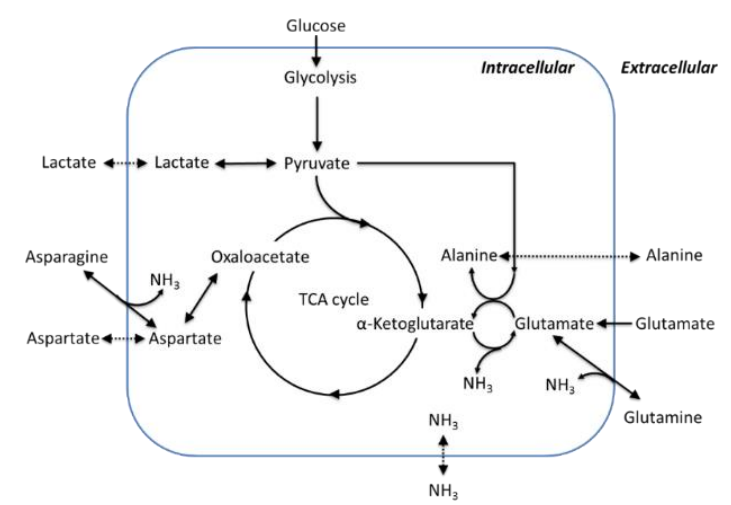

Figure 11. Schematic diagram of pathways related to the fates of lactate and ammonia.

\subsubsection{Optimization of fed-batch cultures}

Optimization of medium and feed formulations, and feeding strategy is an important step in process development. To keep stable nutrient levels, a feed formulation that is balanced with respect to the needs of a cell line over the whole culture period is required. In this study, a glucose based feeding strategy is applied instead of following the prescribed feeding strategy by suppliers in order to obtain a fairer cross comparison between basal media and feeds. This feeding strategy resulted in overfeeding of amino acids by Actifeed $\mathrm{A} / \mathrm{B}$ on both clones (Figure 6) due to an imbalance in the feed. Notably, overfeeding of amino acids also occurred when following the protocols prescribed by the suppliers ${ }^{47}$. This study demonstrates that $\mathrm{CHO}$ cells may display a high variation in specific glucose consumption in different basal medium-feed combinations and during different phases. The increase of the specific glucose consumption is related to the increase of cell size. Moreover, the variation of the specific glucose consumption rate does not always correlate with the specific amino acid consumption rate, in other words, they do not shift up or down at the same time and to the same extent. Thus, as was done in other studies ${ }^{15,49}$, it is better to add glucose through a separate feed from the amino acids. Part of the variation in glucose consumption rate may be caused by uncontrolled glucose levels. The high glucose levels were shown to induce inefficient use of glucose, thus result in higher glucose consumption rate and higher lactate production rate ${ }^{47}$. Hence, control of glucose concentration at low values may reduce the variation and keep feeds more balanced throughout the process.

For all tested culture conditions the ratios of specific amino acids consumption rates changed upon transition from the growth phase to the stationary phase, implying that the composition of amino acids in the feed should also be changed accordingly. 
However, within the growth and the stationary phases, the behavior of the cells is fairly constant. Therefore, it would be possible to have two different feeds for the growth and the stationary phases. To our knowledge, a feed system design to meet different nutrient requirements for different culture phases has not been reported. Another feeding strategy would be to separate feed streams with nutrients that have the same ratio of specific uptake rates (eg. essential amino acids, which ratios of requirement do not change much) in the same stream. Changes in specific consumption rates during, for example a phase transition, can then be addressed by adjusting the feed rates for the separate feeds. An extra challenge for feed design is the solubility of different nutrients. Using more feed streams may circumvent this problem and make the feeds more concentrated and balanced. To solve this the amino acids could also be added as di- or tripeptides. It was shown that amino acids supplemented in the form of a dipeptide or tripeptide improved their solubility, yet resulted in similar culture effects ${ }^{70}$.

The outcome of clone generation at this moment remains unpredictable. Intensive screening of clones and media is therefore still the most common industrial practice. It is shown in this study that although 2 clones were generated in the same way from the same parental cell line, they can still have distinct metabolic behavior for the different medium-feed systems. Only for the essential amino acids in the growth phase, ratios of the specific consumption rates were similar for the two clones (Figure 8 A\&C). Thus, for essential amino acids a single feed can be used for both clones in the growth phase.

\subsection{Conclusion}

The aim of this work is to investigate the effects of different basal media and feeds on cell growth, metabolism, physiology, and mAb production for two differentially behaving clones derived from the same parental cell line. The two clones show different metabolic and physiological responses for the different medium-feed combinations that were applied. The best $\mathrm{mAb}$ product titer $(1.2 \mathrm{~g} / \mathrm{L})$ is obtained with ActiCHO basal medium and feeds. For all the tested conditions, the specific consumption rates for amino acids and glucose are different for the growth and stationary phase. Also the ratio of specific uptake rates are different in the different phases. However, the ratios of the consumption rates for amino acids within a phase are fairly constant for each of the two clones. Cells grown in cultures fed by Actifeed A/B show an increase in cell size during the stationary phase. The size increase correlates with an increase in specific glucose consumption rate in the stationary phase, whereas for all other conditions the specific glucose consumption rate decreases in the stationary phase. The increase of specific glucose consumption rate in the stationary phase of the cultures fed by Actifeed A/B leads to overfeeding of the 
other nutrients, since the feeding is based on glucose. The result of this study can help to design better feed formulation and feeding strategy. 



\title{
Chapter 3
}

\author{
Metabolic characterization of a $\mathrm{CHO}$ cell \\ size increase phase in fed-batch cultures
}




\begin{abstract}
:
Normally, the growth profile of a $\mathrm{CHO}$ cell fed-batch process can be divided into two main phases based on changes in cell concentration, being an exponential growth phase and a stationary (non-growth) phase. In this study, an additional phase is observed during which the cell division comes to a halt but the cell growth continues in the form of an increase in cell size. The cell size increase (SI) phase occurs between the exponential proliferation phase (also called the number increase or NI phase) and the stationary phase. During the SI phase, the average volume and dry weight per cell increase threefold linearly with time. The average mAb-specific productivity per cell increases linearly with the cell volume and therefore is on average two times higher in the SI phase than in the NI phase. The specific essential amino acids consumption rates per cell remain fairly constant between the NI and the SI phase, which agrees with the similar biomass production rate per cell between these two phases. Accumulation of fatty acids and formation of lipid droplets in the cells are observed during the SI phase, indicating that the fatty acids synthesis rate exceeds the demand for the synthesis of membrane lipids. A metabolic comparison between $\mathrm{NI}$ and SI phase shows that the cells with a larger size produce more $\mathrm{mAb}$ per unit of $\mathrm{O}_{2}$ and nutrient consumed, which can be used for further process optimization.
\end{abstract}

\title{
Xiao Pan ${ }^{a^{*}}$
}

Ciska Dalm ${ }^{\mathrm{b}}$

René H. Wijffels a, c

Dirk E. Martens ${ }^{a}$

\footnotetext{
a Bioprocess Engineering, Wageningen University, PO Box 16, 6700 AA, Wageningen, the Netherlands b Synthon Biopharmaceuticals BV, Upstream Process Development, PO Box 7071, 6503 GN, Nijmegen, the Netherlands

cFaculty of Biosciences and Aquaculture, Nord University, N-8049, Bodø, Norway
}

Appl Microbiol Biotechnol (2017) 101:8101-8113 


\subsection{Introduction}

Chinese Hamster Ovary $(\mathrm{CHO})$ cells are the predominant host for the production of therapeutic monoclonal antibodies (mAbs). At the moment, fed-batch is the most common industrial process for $\mathrm{CHO}$ cell cultures, due to its ease of operation and flexibility. Three different culture phases can normally be discerned in a fed-batch culture: an exponential growth phase, a stationary phase, and a death phase $e^{60,71,72}$. The transitions between these phases are caused by the changes in culture conditions (e.g. nutrient depletion and/or waste accumulation). The transition from the growth to the stationary phase determines the maximum viable cell density (VCD), whereas the transition from stationary to death phase determines the culture longevity. These collectively determine the integral viable cell density (IVCD) which is positively correlated with the final $\mathrm{mAb}$ product titer. A better understanding of the underlying mechanisms causing these transitions can be used to develop cultivation processes in which cells grow to higher densities or to remain viable for a longer period of time, which both will result in an increased $\mathrm{mAb}$ volumetric productivity. Many studies have compared the metabolic and physiological states of different $\mathrm{CHO}$ cell culture phases. The growth phase is characterized by higher specific rates of glycolysis and glutaminolysis and, as a result, also by a higher lactate production rate. During the stationary phase, the metabolism shifts towards a more efficient use of substrates, represented by a lower metabolic flux through glycolysis, a higher flux through the TCA cycle, and often the consumption of lactate. Martínez et al. ${ }^{61}$ showed that a $\mathrm{CHO}$ cell line grown in a batch culture had a six times higher energy efficiency when consuming lactate as compared to the situation where lactate was produced. Using isotopic labeling in combination with metabolic flux analysis, Sengupta et al. ${ }^{72}$ and Templeton et al..$^{73}$ both reported higher metabolic fluxes through the pentose phosphate pathway (PPP) and TCA cycle during the stationary phase as compared to the growth phase of $\mathrm{CHO}$ cell cultures. Furthermore, Templeton et al. ${ }^{73}$ showed that the high PPP and TCA activities are associated with peak mAb production. Results from Wahrheit et al. ${ }^{74}$ suggested that the metabolic shift in different culture phases of $\mathrm{CHO}$ cells is controlled by glycolytic regulation which affects the intracellular pyruvate availability. Ahn and Antoniewicz ${ }^{75}$ reported a higher fatty acids biosynthesis rate than needed by the cells during the stationary phase of $\mathrm{CHO}$ cell cultures. Despite that these studies have contributed to the fundamental understanding of $\mathrm{CHO}$ cell metabolism and resulted in improved process performances, the mechanisms underlying the phase are not yet fully understood, due to the complexity of media and feeds used and the highly dynamic nature of the current fed-batch processes.

In a previous study on the selection of basal media and feeds for $\mathrm{CHO}$ cell fed-batch cultures $^{8}$, it was observed that a cell size increase occurred after the exponential growth phase when ActiCHO feed A/B (GE Healthcare) was used but not when Efficient feed A, B, and C $\left(\right.$ Gibco $\left.^{\mathrm{TM}}\right)$ were used. The cell size increase correlated with 
higher final product titers. The physiological state of the larger cells and the cause of the size increase were not clear. The aim of this paper is to characterize the metabolic changes that occur upon the cell size increase in order to obtain more insight in the underlying mechanisms that cause the cell size increase and identify possible implications for process design. The metabolic changes are characterized in terms of biomass composition, specific consumption and production rates, and overall metabolic flux distributions using flux balance analysis (FBA).

\subsection{Materials and Methods}

\subsubsection{Cell line and pre-culture}

A suspension $\mathrm{CHO}^{\mathrm{BC}}{ }^{\circ}$ cell clone (BC-P, provided by Bioceros Holding BV, Utrecht, NL) producing a recombinant immunoglobulin G1 (IgG1) was used in this study. Cells were thawed from a working cell bank and maintained in ActiCHO-P medium (GE Healthcare, France) supplemented with $4 \mathrm{mM}$ glutamine (Gibco ${ }^{\mathrm{TM}}$, Paisley, Scotland, UK) and 0.5\% Anti-clumping agent (Gibco ${ }^{\mathrm{TM}}$ Paisley, Scotland, UK). 200 $\mu \mathrm{g} / \mathrm{mL}$ Zeocin ${ }^{\mathrm{TM}}$ and $5 \mu \mathrm{g} / \mathrm{mL}$ Blasticidin (both from Gibco $^{\mathrm{TM}}$, Grand Island, NY, USA) as selection reagents were added during the pre-cultures. Cells were maintained in a $125 \mathrm{~mL}$ un-baffled shake flask (Corning, NY, USA) with a $25 \mathrm{~mL}$ working volume in a $\mathrm{CO}_{2}(8 \%)$ and temperature $\left(37^{\circ} \mathrm{C}\right)$ controlled incubator. The maintained cultures were sub-cultured every 3 days to $2 \times 10^{5}$ viable cells $/ \mathrm{mL}$. The seed train was scaled up through $2 \mathrm{~L}$ and $20 \mathrm{~L}$ rocking bag systems. During the seed train scale-up, the medium described above was used but without the two selection reagents.

\subsubsection{Fed-batch process}

Triplicate fed-batch cultures were conducted in $10 \mathrm{~L}$ bioreactors (Sartorius Stedim Biotech, France) controlled by BIOSTAT ${ }^{\circledR}$ B-DCU II. Each bioreactor was inoculated with a starting density of $3 \times 10^{5}$ viable cells $/ \mathrm{mL}$ at a starting volume of $5 \mathrm{~L}$. Culture temperature was controlled at $37^{\circ} \mathrm{C}$, dissolved oxygen (DO) was controlled at $40 \%$ by enriched $\mathrm{O}_{2}$ flow, $\mathrm{pH}$ was controlled at 7.2 by using base and $\mathrm{CO}_{2}$. From day 3 onward, feeds were added to each bioreactor daily. First, the glucose concentration was measured. If the concentration was lower than $18 \mathrm{mM}$, a $45 \%$ (w/w) glucose solution was added as one bolus to reach a glucose concentration of $28 \mathrm{mM}$ in the reactor. Next, 4.5\% (v/v) ActiCHO feed A, also containing about $500 \mathrm{mM}$ glucose amongst other nutrients, and $0.45 \%$ (v/v) ActiCHO feed B (both from GE Healthcare, USA) per culture volume per day were fed to each reactor. Simethicone antifoam solution was added to each bioreactor prior to inoculation and during the culture when needed. 


\subsubsection{Sampling and analysis}

For each bioreactor, a-15 mL sample was taken daily before and after the feed addition. Total cell density, viable cell density, and cell diameter were measured using a CedexHiRes ${ }^{\circledR}$ analyzer (Roche, Switzerland). Off-line $\mathrm{pH}, \mathrm{pCO}_{2}$, glucose concentration, lactate concentration, ammonium concentration and osmolality were measured by a Nova FLEX analyzer (Nova Biomedical, USA). The remaining sample was spun down at $3345 \times \mathrm{g}$ for $15 \mathrm{~min}$ and stored at $-20^{\circ} \mathrm{C}$ for later analysis. On culture day 4,7 , and 10, biomass samples were taken from each bioreactor containing 300 million cells per sample. The samples were spun down at $500 \times \mathrm{g}$ for $10 \mathrm{~min}$ and resuspended in PBS solution (Lonza, Switzerland). Next, the viable cell density was measured again, and each sample was aliquoted into six $15 \mathrm{~mL}$ centrifuge tubes with each tube containing 50 million viable cells. The tubes were spun down at $500 \times \mathrm{g}$ for $10 \mathrm{~min}$ again after which the PBS supernatant was discarded and the wet cell pellets were stored at $-20^{\circ} \mathrm{C}$ for later biomass analysis.

Total soluble cellular protein was determined using Lowry Bio-Rad DC Protein assay kit (Bio-Rad, NL). Bovine serum albumin (BSA, Sigma-Aldrich) was used as a reference standard. The extraction, separation, and quantification of triacylglyceride (TAG) and polar lipids were performed as described by Breuer et al. ${ }^{76}$ using the sample preparation method 2. Lipid droplets in $\mathrm{CHO}$ cells were stained with BODIPY 505/515 (Invitrogen Molecular Probes, Carlsbad, CA) and visualized using a confocal laser scanning microscope (LSM510; Carl Zeiss, Jena, Germany), as described by Cooper et al. ${ }^{77}$. Total cellular carbohydrate content was measured according to the DuBois' method ${ }^{78}$. A glucose solution (Sigma-Aldrich) was used as a reference standard. Cell dry weight (DW) was calculated based on the difference in weight of the tube with the 50 million freeze-dried cells and the pre-weighed centrifuge tube itself.

Compositions of the spent medium including extracellular amino acids, sugars, and organic acids were quantified using NMR (Spinnovation Biologics BV, Oss, NL). IgG1 titer was quantified by Protein-A Chromatography (Agilent, 5069-3639). The Nglycans were quantified by Hydrophilic Interaction Chromatography (HILIC UPLC). A dextran calibration ladder standard (Waters) solution was used to identify the glucose unit of the measured N-glycans. Both mAb quantification and N-glycan analysis were developed by Bioprocess engineering group of Wageningen University.

\subsubsection{Average specific metabolic rates}

The average specific metabolic rates were calculated for the NI and the SI phase, respectively. Day 0 and 1 were not considered in calculating the average specific rates for the NI phase, due to a metabolic adaptation period just after inoculation. 
The average specific production rate of antibody was calculated by averaging the daily specific rates during both the NI and the SI phases. The following equation is used to calculate the specific production rate of a compound $x$, as described in Chapter $2^{8}$ :

$$
M_{x}(t)-M_{x}(0)-V_{f} \times C_{f}=q_{x} \cdot \int_{0}^{t} X_{V C} d t \quad \text { (Equation 1) }
$$

where $M_{x}(\mathrm{mg} ; \mathrm{mmol})$ is the total amount of compound $\mathrm{x}$ in a culture, $\mathrm{X}_{\mathrm{vc}}$ is the number of viable cells in the reactor, $V_{f}\left(\mathrm{~mm}^{3}\right)$ is the total volume of feed added, $C_{f}$ $(\mathrm{mM})$ is the concentration of compound $\mathrm{x}$ in the feed, and $\mathrm{q}_{\mathrm{x}}\left(\mathrm{mg} \cdot \mathrm{cell}^{-1} \cdot \mathrm{day}^{-1}\right.$; $\left.\mathrm{mmol} \cdot \mathrm{cell}^{-1} \cdot \mathrm{day}^{-1}\right)$ is the cell-specific production rate of the compound $\mathrm{x}$. When the rates are calculated based on cell volume, $X_{\mathrm{vc}}\left(\mathrm{mm}^{3}\right)$ presents the volume of viable cells in a culture and $\mathrm{q}_{x}\left(\mathrm{mg} \cdot \mathrm{mm}^{-3} \cdot \mathrm{day}^{-1}\right.$ or $\mathrm{mmol} \cdot \mathrm{mm}^{-3} \cdot$ day $\left.^{-1}\right)$ is the cell volumespecific production rate of compound $x$. The average specific production rates $(q)$ of glucose, amino acids, and organic acids were obtained from a plot of the total amount of production/consumption against the total integral viable cell number (or total integral viable cell volume) using linear regression. Positive values indicate production while negative values indicate consumption. The specific cell growth rate $\left(\mu_{\mathrm{N}} ;\right.$ day $\left.^{-1}\right)$ during the NI (exponential growth) phase is given by:

$$
\mu_{N I}=\ln _{X_{V C}(0)} / x_{t} \quad \text { (Equation 2) }
$$

where $\mathrm{X}_{\mathrm{vc}}$ is the total number of viable cells in the reactor. During the SI phase in which the cell concentration is constant and the cell volume increases linearly with time, the cell-specific production rate of biomass (qsi; day $\left.{ }^{-1}\right)$ is calculated by:

$$
\mathrm{q}_{S I}=\frac{M(t e)-M(t s)}{t e-t s} / X_{V C}
$$

(Equation 3)

where $M(t e)$ and $M(t s)$ are the amounts of biomass in a reactor at the end (te) and the start (ts) of the SI phase. In principle, the unit of M is gram dry weight. However, to compare the growth in the SI phase to the exponential growth in the NI phase, the amount of biomass is still expressed in cell number by dividing the dry weight by the dry weight of a cell in the NI phase. In this way, the specific biomass production rate in the SI phase can still be compared to the specific growth rate in the NI phase. The specific growth rate in the NI phase and the specific biomass production rate in the SI phase are used as input values for the subsequent flux balance analysis.

\subsubsection{Flux balance analysis (FBA) and flux variability analysis (FVA)}

A basic model of $\mathrm{CHO}$ cell primary metabolism was developed starting from an earlier published basic model for mammalian cells ${ }^{79}$. The reactions in the model were cross-checked with the iCHOv1 model ${ }^{80}$. Several modifications were made based on the Kyoto Encyclopedia of Genes and Genomes (KEGG) database, the iCHOv1 
model, and the measurements done in this study. A Chi-square test was used to check whether the nitrogen balances closed for both phases. The FBA was conducted using the constraint-based reconstruction and analysis (COBRA 2.0) Toolbox ${ }^{81}$, using MATLAB software (The MathWorks, Inc.). Geometric FBA ${ }^{82}$ was performed to find a unique solution for the underdetermined parts of the metabolic model. The growth rate was used as an objective function for the model. The measured cell-specific production rates were used as inputs for FBA. To study the possible range of flux values that results in the same optimal growth rate while still meeting all constraints, flux variability analysis (FVA) was performed using the COBRA Toolbox.

\subsection{Results}

\subsubsection{Cell growth}

Based on cell concentration, the growth curve of $\mathrm{CHO}$ cells normally consists of an exponential cell proliferation phase, a stationary phase, and a death phase. In this study, an additional cell size increase phase is reported (Figure 1), resulting in four phases for this $\mathrm{CHO}$ cell fed-batch process being: (i) A phase in which the cell concentration increases exponentially (day 0 to 4 ) and the volume per cell remains constant (the number increase or NI phase). (ii) A phase in which the cell size increases nearly linear $\left(\mathrm{R}^{2}=0.99\right)$ in time (day 4 to 8 ) and the cell concentration stays approximately constant (the size increase or SI phase). (iii) A short stationary phase (day 8 to 10) in which the biomass concentration remains approximately constant and the culture viability is always higher than $80 \%$. (iv) A death phase (day 10 to 12) in which the viability declines and is always lower than $80 \%$. Since the observed change from growth in cell concentration to growth in cell size has not been reported in regular fed-batch processes and can have consequences for process development, this study mainly focuses on the NI phase and the SI phase. 

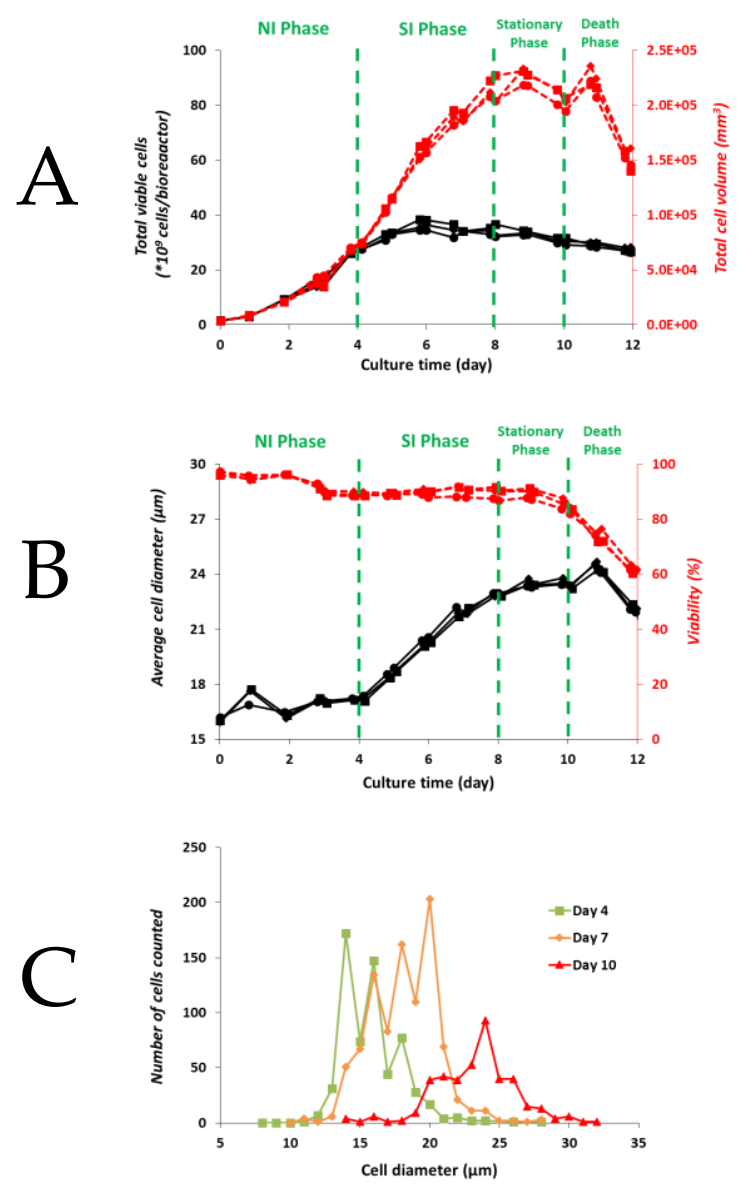

Figure 1. (a) Total number of viable cells $\left(\times 10^{9}\right.$, solid lines) and total volume of viable cells $\left(\mathrm{mm}^{3}\right.$, dotted lines), and (b) average cell diameter ( $\mu \mathrm{m}$, solid lines) and viability (\%, dotted lines) of reactor 1 (closed circles), 2 (closed diamonds), and 3 (closed squares). Cultures were divided into a number increase (NI) phase, a size increase (SI) phase, a stationary phase, and a death phase. (c) Cell diameter ( $\mu m)$ distribution during a representative fed-batch culture on day 4 (closed squares), 7 (closed diamonds), and 10 (closed triangles)

\subsubsection{Metabolites profiles}

To identify possible limiting or inhibitory compounds and to characterize metabolism in the different phases, extracellular concentrations of substrates and products were measured. Concentrations of extracellular glucose, lactate, and ammonium are shown in Figure 2a. The $45 \%$ glucose solution is added on culture day $3,7,8$, and 10 when the glucose concentration is lower than $18 \mathrm{mM}$, as described in materials and methods. Next to this, the ActiCHO feed A\&B are added daily. On the days other than $3,7,8$, and 10 , the increases in culture glucose concentrations were caused by the addition of ActiCHO feed A only. Using this feeding strategy, the glucose concentration in each bioreactor stayed above $5 \mathrm{mM}$ throughout the fedbatch cultures. Furthermore, since the ActiCHO feed A also contains glucose, the culture glucose concentration after feeding can be higher than $28 \mathrm{mM}$. Lactate is produced during the NI phase (day 0-4) until nearly $30 \mathrm{mM}$ and is consumed at a 
lower rate during the SI phase and stationary phase (day 4-10), followed by production again during the death phase (day 10-12). Ammonium concentrations stay constant at values lower than $5 \mathrm{mM}$ until culture day 7 and start to increase gradually thereafter up to $15 \mathrm{mM}$ on day 12. Production of formic acid (up to $7 \mathrm{mM}$ at day 10) and acetic acid (up to $15 \mathrm{mM}$ at day 10) are observed in the cultures (Figure 2b). Succinic acid is present in the basal medium and is consumed during the culture (Figure $\mathbf{2 b}$ ). None of the essential amino acids is depleted during the fedbatch cultures (Figure 2c). The gradual increase in their concentrations after day 3 is caused by the feed addition, since these essential amino acids cannot be made by $\mathrm{CHO}$ cells from the other compounds. Note that for some of the non-essential amino acids the cell may not be able to synthesize them fast enough to support growth making them essential for a robust process, consequently, they also need to be present in the medium and feed. For some of the non-essential amino acids, asparagine, glutamine, and cyst(e)ine (cysteine is measured in the form of cystine in this study) are consumed during the NI phase and are depleted around day 4 (Figure 2d). Glutamine, which is not present in the feed, is produced after day 7. Cyst(e)ine concentration increases after day 5 .
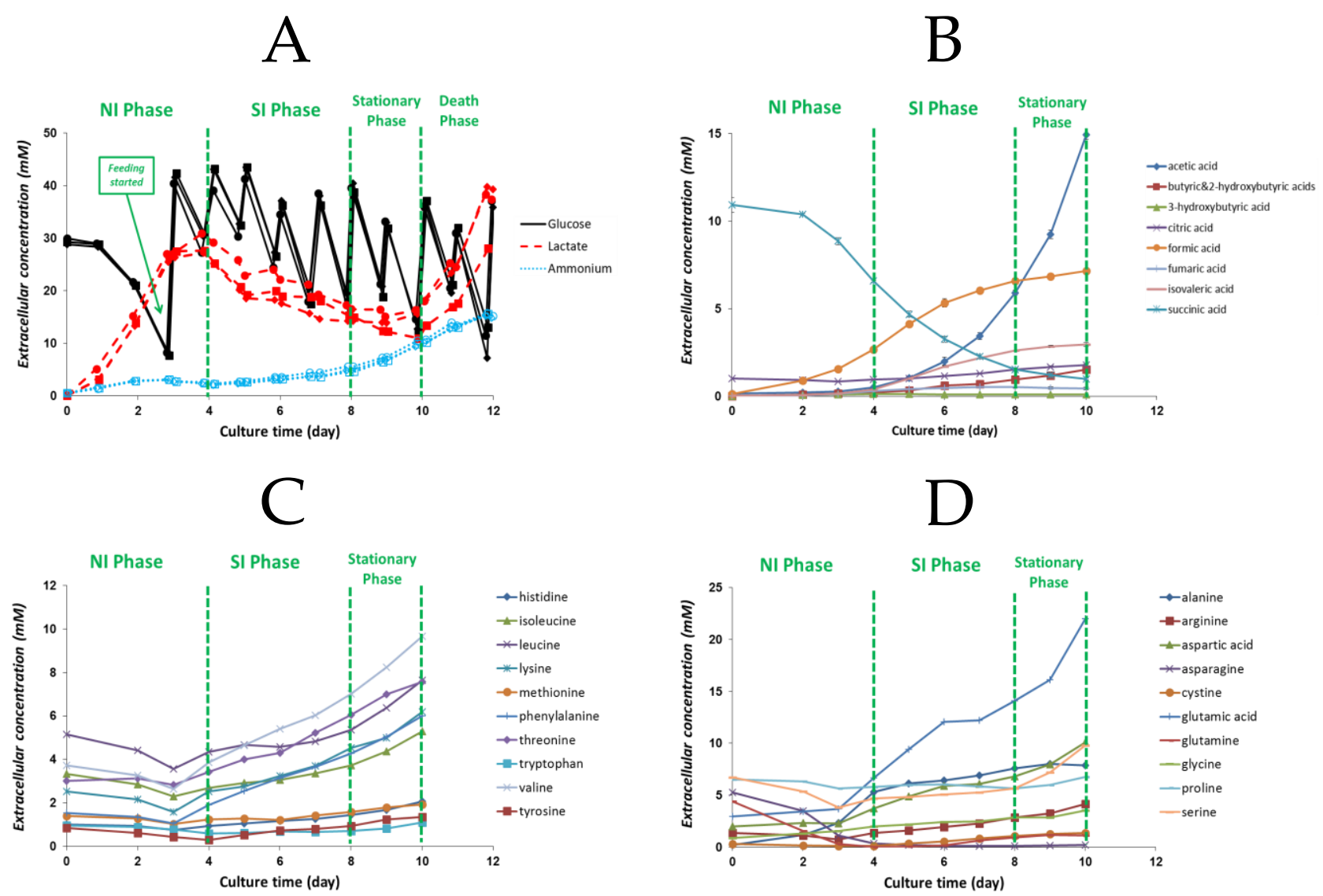

Figure 2. (a) Extracellular glucose (solid lines), lactate (dotted lines with closed marks), and ammonium (dotted lines with open marks) concentrations ( $\mathrm{mM}$ ) of reactor 1 (circles), 2 (diamonds), and 3 (squares). (b) Extracellular concentrations of measured organic acids. (c) Extracellular concentrations of essential amino 
acids. (d) Extracellular concentrations of non-essential amino acids. Feeding started on day 3. For glucose, lactate, and ammonium, samples were taken daily before and after feed addition. For the other organic acids and amino acids, samples were taken daily before feed addition. Error bars for all graphs indicate the standard deviation for the triplicate bioreactors

\subsubsection{Cell-specific rates vs. volume-specific rates}

In order to obtain insight into the relationship between cell volume and the specific consumption and production rates of nutrients and products, including $\mathrm{mAb}$, their metabolic rates were calculated and compared in this section. The rates are calculated on a per cell and per cellular volume basis for the NI and the SI phase, respectively.

The average specific mAb productivities calculated based on cell-volume and cellnumber are shown in Figure 3a. Average specific productivity expressed per cell is

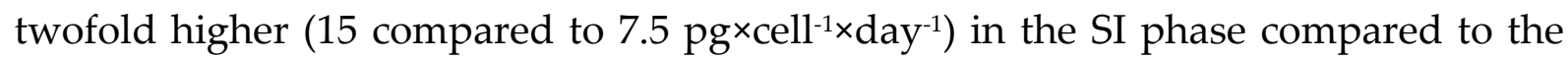
NI phase. When calculated per cell volume, the specific productivities in the two phases are not significantly different, indicating that the mAb productivity of a cell is related to its volume. This becomes clearer when the productivity per cell is plotted against the cellular volume, which results in a linear correlation between average cell volume and specific mAb productivity as shown in Figure 3c. The specific glucose consumption based on cell number shows only a slight decrease ( 4.5 to $4.1 \mu \mathrm{mol} \times 10^{-6}$ cells $\times$ day $\left.^{-1}\right)$ from the NI to the SI phase. The decrease is larger $\left(1.9\right.$ to $0.8 \mu \mathrm{mol} \times \mathrm{mm}^{-}$ ${ }^{3} \times$ day $^{-1}$ ) when the rate is calculated based on cell volume. Thus, the specific glucose consumption rate is related more to the cell number and less to the cell volume. Lactate is produced during the NI phase at a high rate $\left(3 \mu \mathrm{mol} \times 10^{-6}\right.$ cells $\times$ day $^{-1} ; 1.3$ $\left.\mu \mathrm{mol} \times \mathrm{mm}^{-3} \times \mathrm{day}^{-1}\right)$ and is consumed during the SI phase at a much lower rate $(0.25$ $\mu \mathrm{mol} \times 10^{-6}$ cells $\times \mathrm{day}^{-1} ; 0.05 \mu \mathrm{mol} \times \mathrm{mm}^{-3} \times \mathrm{day}^{-1}$ ) $($ Figure 4$)$. The cell number-specific and cell volume-specific consumption rates of ten essential amino acids during the NI and the SI phase are shown in Figure 5. When the consumption rates are expressed based on cell number, six out of ten essential amino acids do not show a significant difference between the two phases. However, as the cell volume increases in the SI phase, the cell volume-specific consumption rates of all the essential amino acids show significant drops. This implies that the consumption of the essential amino acids is more related to the cell number than to the cell volume. 

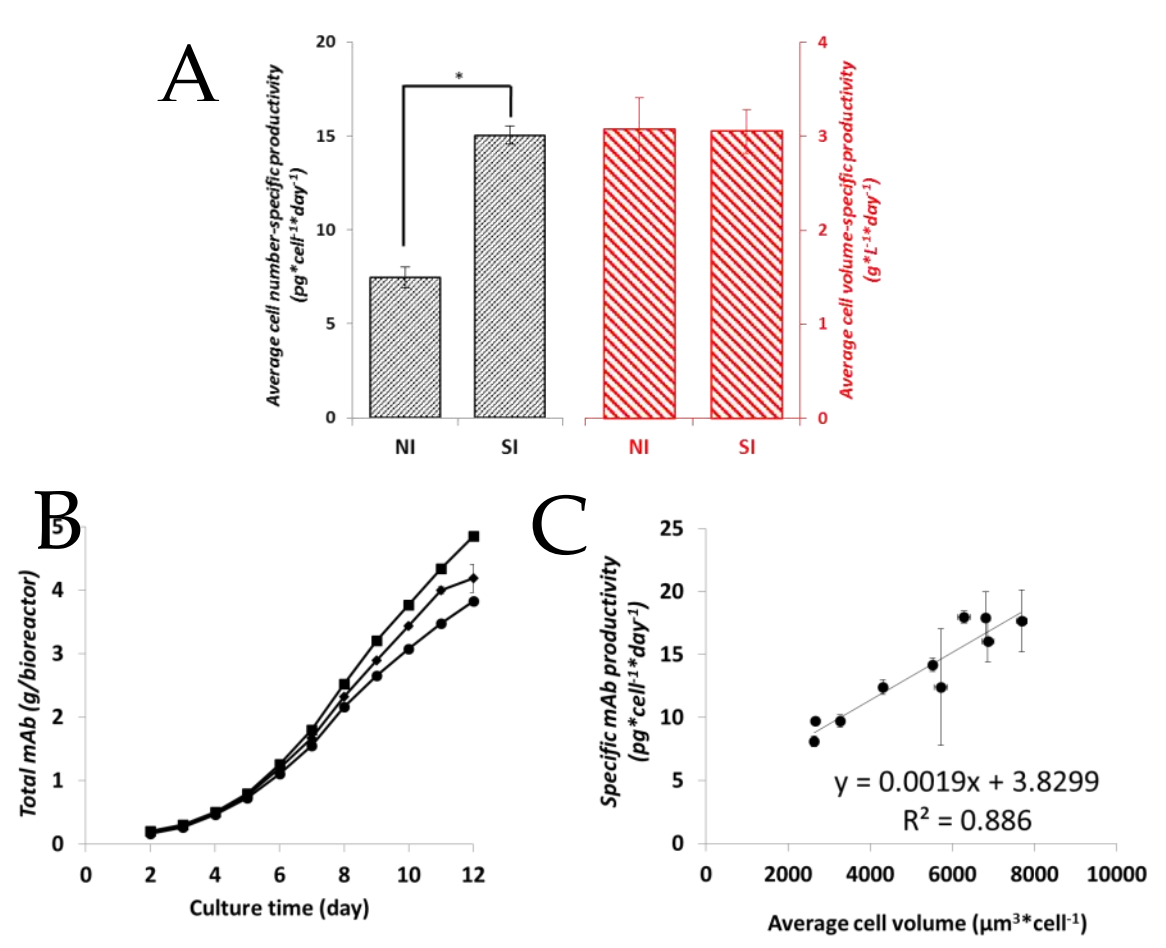

Figure 3. (a) Average cell number-specific $\left(p g \times c e l l^{-1} \times d a y^{-1}\right)$ and average cell volume-specific $\left(g \times L^{-1} \times d a y^{-1}\right)$ $m A b$ productivity compared between the number increase (NI) and size increase (SI) phase of the cultures. *: significantly different $(P<0.05)$. (b) Total $m A b$ produced $(g)$ in reactor 1 (closed circles), 2 (closed diamonds), and 3 (closed squares). (c) Plot of specific mAb productivity $\left(\mathrm{pg} \times \mathrm{cell}^{-1} \times\right.$ day $\left.^{-1}\right)$ against cell volume $\left(\mu^{3}\right)^{3}$. Calculation was done per day from day 2 through day 12. Error bars of graph (a) and (c) show the standard deviation of triplicate fed-batch cultures. Error bars of graph (b) show the standard deviation of triplicate measurements for each fed-batch culture

\subsubsection{Biomass composition}

The composition of biomass with respect to protein, fatty acids, carbohydrate, RNA, and DNA is shown in Table I as a percentage of the cell dry weight (DW). The amino acid composition of the cellular protein and the fatty acids composition of the lipids stayed constant during the fed-batch culture (the average values are given in ESM_3 Table S1 \& S2 of the online published data). The cell DW increased linearly with the cell volume over the culture time, from $770 \mathrm{pg} / \mathrm{cell}$ on day 4 to $2440 \mathrm{pg} / \mathrm{cell}$ on day 10 (Table I). Furthermore, the relative composition of the biomass shifted during the cultures. The protein content in the biomass decreased from $74.5 \%$ to $67 \%$ whereas the fatty acid content increased from $7.2 \%$ to $10.3 \%$ from culture day 4 until culture day 10. A microscopic image (Figure 6) shows a clear accumulation of lipid droplets during the SI phase. These droplets are not composed of triacylglyceride (TAG) (data not shown). The sum of all biomass components adds up to around $90 \%$ of the cell DW. 
Table I. CHO cell biomass composition during the fed-batch cultures

\begin{tabular}{|c|c|c|c|c|c|}
\hline Biomass composition & Day 4 & Day 7 & Day 10 & Hybridoma $^{83}$ & Hybridoma $^{84}$ \\
\hline Protein $(\%)$ & $\begin{array}{r}\mathbf{7 4 . 5} \\
\pm 1.0\end{array}$ & $\begin{array}{r}\mathbf{7 0 . 0} \\
\pm 1.2\end{array}$ & $\begin{array}{r}\mathbf{6 7 . 0} \\
\pm 1.0\end{array}$ & 72.9 & 70.6 \\
\hline Fatty acids (\%) & $\begin{array}{c}7.2 \\
\pm 0.2\end{array}$ & $\begin{array}{c}\mathbf{8 . 4} \\
\pm 0.3\end{array}$ & $\begin{array}{l}\mathbf{1 0 . 3} \\
\pm 0.1\end{array}$ & $13.5^{b}$ & $9.7^{b}$ \\
\hline Carbohydrate (\%) & $\begin{array}{c}\mathbf{4 . 0} \\
\pm 0.1\end{array}$ & $\begin{array}{c}\mathbf{3 . 8} \\
\pm 0.2\end{array}$ & $\begin{array}{c}\mathbf{4 . 1} \\
\pm 0.1\end{array}$ & 3.5 & 7.1 \\
\hline $\mathbf{R N A}^{\mathrm{a}}(\%)$ & 6.0 & 5.6 & 5.4 & 3.8 & 5.8 \\
\hline DNA ${ }^{a}(\%)$ & 1.6 & 0.8 & 0.6 & 1.4 & 1.4 \\
\hline Total $(\%)$ & $\begin{array}{l}93.1 \\
\pm 1.4\end{array}$ & $\begin{array}{l}\mathbf{8 8 . 6} \\
\pm 1.7\end{array}$ & $\begin{array}{l}87.4 \\
\pm 1.2\end{array}$ & 95.1 & 94.6 \\
\hline $\begin{array}{c}\text { Cell dry weight } \\
\text { (pg/cell) }\end{array}$ & $\begin{array}{l}770 \\
\pm 47\end{array}$ & $\begin{array}{c}\mathbf{1 5 3 0} \\
\pm 77\end{array}$ & $\begin{array}{c}\mathbf{2 4 4 0} \\
\pm 86\end{array}$ & 250 & 470 \\
\hline $\begin{array}{c}\text { Cell volume } \\
\left(\mathrm{mm}^{3} / 10^{6} \text { cells }\right)\end{array}$ & $\begin{array}{c}\mathbf{2 . 7} \\
\pm 0.02 \\
\end{array}$ & $\begin{array}{c}\mathbf{5 . 5} \\
\pm 0.16 \\
\end{array}$ & $\begin{array}{c}\mathbf{6 . 9} \\
\pm 0.14\end{array}$ & N/R & 1.8 \\
\hline
\end{tabular}

All percentage values are in $\mathrm{w} / \mathrm{w} \%$

a Not measured in this study, data were calculated by assuming a constant DNA content per cell and a constant ratio of RNA to protein

b Values for lipid including glycerol

\pm Standard deviation for biological triplicates

$\mathrm{N} / \mathrm{R}$ not reported
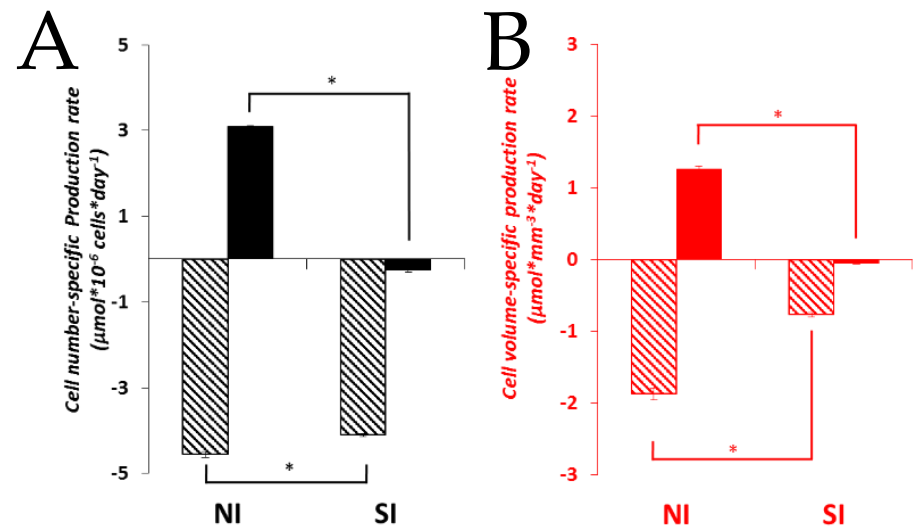

$\mathbb{\mathbb { S } \text { Glucose }}$

Lactate

Figure 4. (a) Cell number-based specific rates $\left(\mu m o l \times 10^{-6}\right.$ cells $\times$ day $\left.{ }^{-1}\right)$ and (b) cell volume-based specific rates $\left(\mu \mathrm{mol} \times \mathrm{mm}^{-3} \times \mathrm{day}^{-1}\right)$ of glucose consumption and lactate production/consumption during the number increase (NI) and size increase (SI) phase of the fed-batch cultures. Positive values indicate production and negative values indicate consumption. Error bars show the standard deviation of triplicate fed-batch cultures. *: significantly different $(P<0.05)$ 

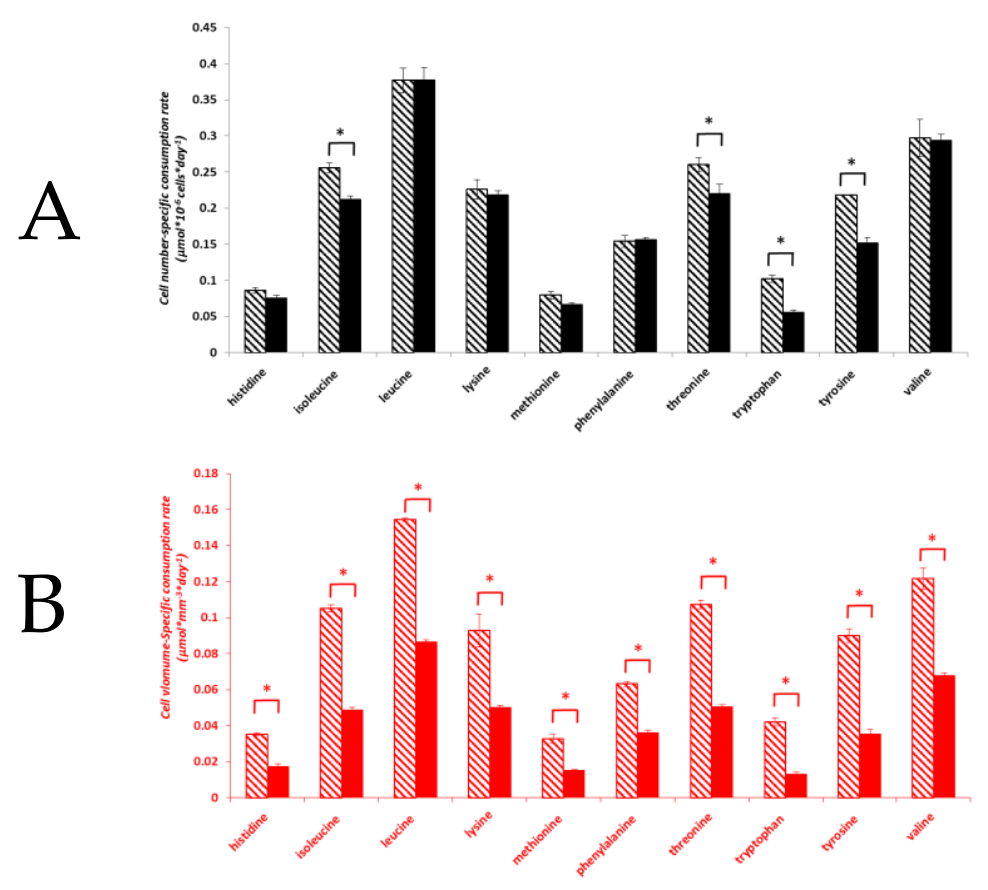

$\mathbb{\$}$ NI Phase $\mathbf{\square}$ SI Phase

Figure 5. (a) Cell number-specific consumption rates $\left(\mu \mathrm{mol} \times 10^{-6}\right.$ cells $\times$ day $\left.{ }^{-1}\right)$ and $(\mathrm{b})$ Cell volume-specific consumption rates $\left(\mu \mathrm{mol} \times \mathrm{mm}^{-3} \times \mathrm{day}^{-1}\right)$ of essential amino acids during the number increase (NI) and size increase (SI) phase of the fed-batch cultures. Error bars show the standard deviation of triplicate fed-batch cultures. *: significantly different $(\boldsymbol{P}<0.05)$
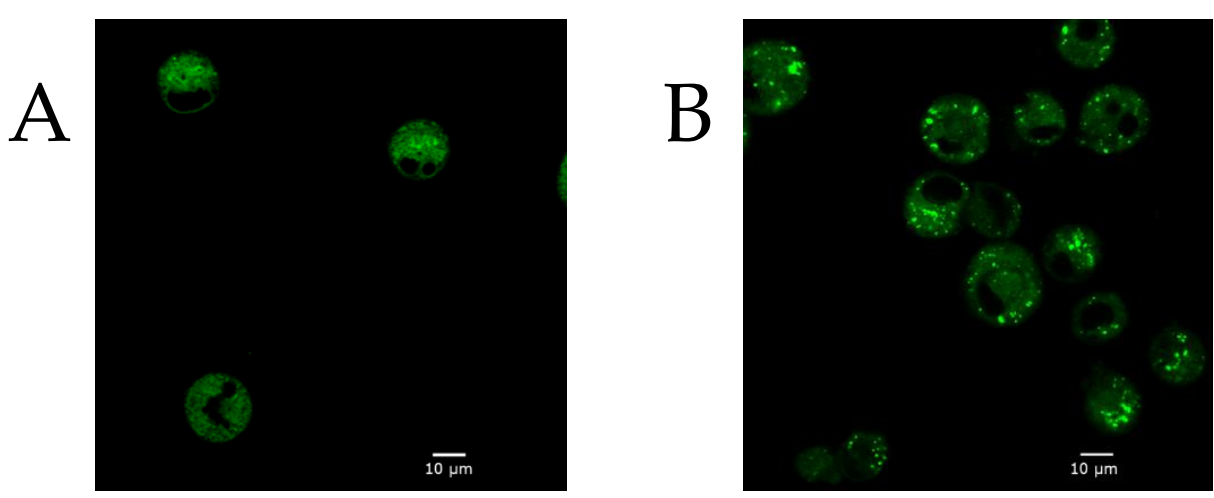

Figure 6. Lipid droplets stained with BODIPY 505/515 (green) during the CHO cell fed-batch on culture day 3 (a) and culture day $6(b)$ 


\subsubsection{Flux balance analysis}

Geometric flux balance analysis (FBA) is carried out to further investigate the shift of the overall metabolic flux distribution from the NI phase to the SI phase. The FBA results for both phases are summarized in Figure 7 . The metabolic fluxes are expressed per cell because the uptake rates of essential amino acids and glucose, and the biomass formation rate are constant on a per cell basis in the NI and SI phase. On a per volume basis rates are continuously decreasing during the SI phase due to the increase in cell volume. During the SI phase, the change in biomass content per cell and in biomass composition is considered in the biomass equation in the FBA model. The flux variability analysis (FVA) shows that the flux variability results in overlapping flux values with respect to the two different phases for the PPP as well as for the TCA cycle, as indicated by the red numbers in Figure 7. This means the value of these fluxes cannot be identified as being different between the two phases, which is further addressed in the discussion. During the NI phase, high fluxes through lactate and alanine production are calculated. During the SI phase, lactate metabolic flux shifts to a consumption and the flux from pyruvate to alanine decreases. Higher $\mathrm{O}_{2}$ consumption and $\mathrm{CO}_{2}$ production fluxes ( 1.5-fold higher) are calculated for the SI phase, which agrees with the higher ATP generation flux in the mitochondria. The ATP is transported to the cytosol through ATP/ADP translocases and used for biomass and product synthesis, and for cell maintenance. The cellular protein formation rate decreases by $25 \%$ whereas the $\mathrm{mAb}$ formation increases by $250 \%$ during the SI phase. In the NI phase, $\mathrm{mAb}$ production is about $1.5 \%$ of the total protein synthesized, whereas in the SI phase this becomes $5 \%$. In the SI phase, the fatty acids synthesis flux is slightly higher and the flux from AcCoA to acetic acid is also higher than in the NI phase.

\subsection{6 mAb N-glycan composition}

The glycosylation profile of a $\mathrm{mAb}$ is routinely analyzed throughout process development to ensure process consistency. In order to investigate whether or not the mAb glycosylation changes as cell size increases, the $\mathrm{N}$-glycan composition of the produced $\mathrm{mAb}$ is measured on culture days 5, 8 and 12 (Figure 8). The relative composition of N-glycans does not show differences between culture day 5 and 8 , indicating that the $\mathrm{mAb}$ glycosylation does not change as cell size increases. Differences on day 12 are observed which is most likely related to cell death and release of degradative enzymes, since the culture viability drops to $60 \%$ on day 12 .

\subsection{Discussion}

In this study using ActiCHO medium as basal medium and Actifeed A and B as feeds, a cell size increase phase was observed during which the cell concentration 
remained approximately constant, while the cell volume increased almost three times. A previous study ${ }^{8}$ showed that the cell size increase in $\mathrm{CHO}$ fed-batch processes is clone and medium/feed dependent. Furthermore, an experiment with the same process and medium system but with a lower feed addition rate, the same extent of cell size increase was observed (data not shown), implying that the cell size increase is not merely caused by nutrient oversupply. However, several non-essential amino acids were depleted around day 4 (Figure $2 \mathbf{d}$ ), which might play a role in the metabolic transition from the NI to the SI phase.
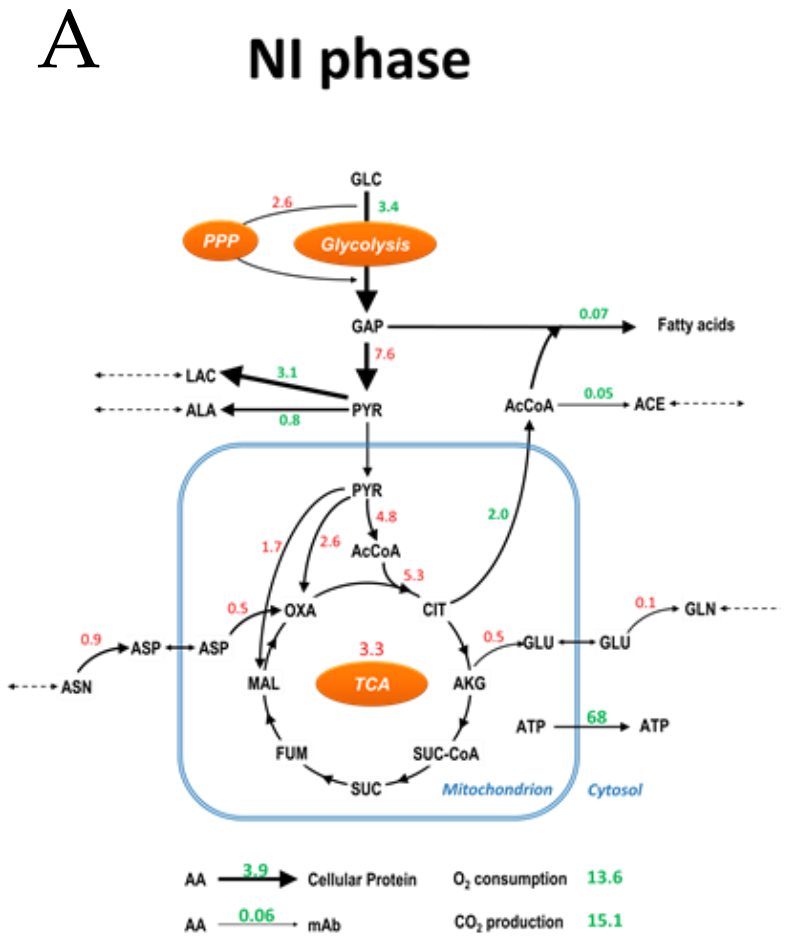

B

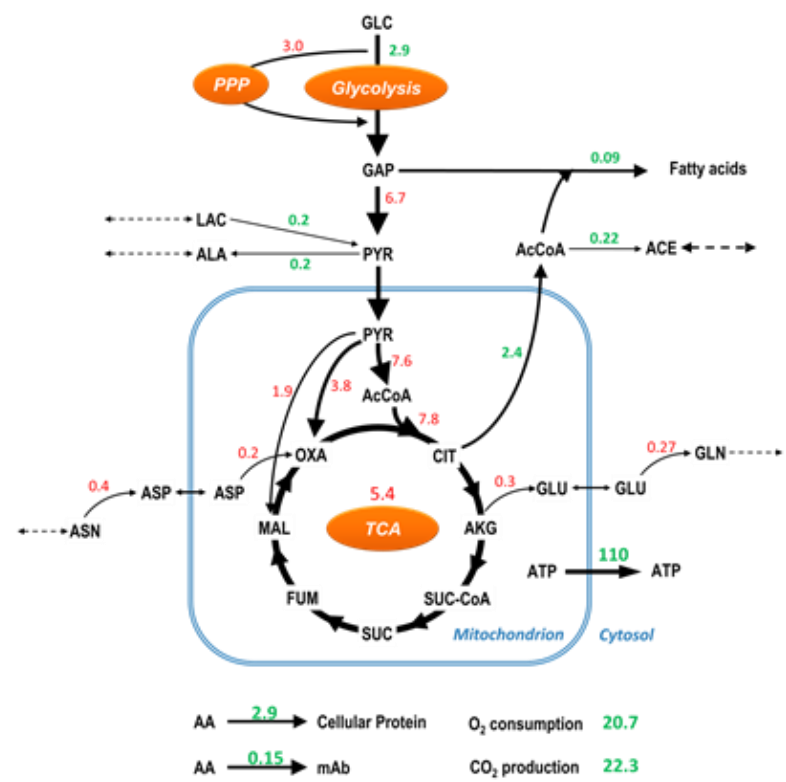

Figure 7. Major metabolic flux changes between (a) the number increase (NI) and size increase (SI) phase during the fed-batch cultures. The line thickness indicates the relative flux changes between the two presented phases. The average flux of the TCA cycle is represented by the average fluxes between the flux from citrate (CIT) to a-ketoglutarate (AKG) and the flux from succinate (SUC) to fumarate (FUM). The colors of the flux values indicate the flux variability result between the two phases. The green values show no overlap of the variable flux values between the two phases whereas the red values show there is overlap. The unit for flux values is mol $\times 10^{-9}$ cells $\times$ day ${ }^{-1}$

The cell diameter distribution during the fed-batch cultures (Figure 1c) showed that the increase in cell diameter occurred for the whole cell population instead of only a sub-population. It was also established in this study that the cell size increase is due to a proportional increase in biomass content (Table I), rather than due to a cell swelling effect through the uptake of water and salts. The cell DW measurement resulted in higher values (770 pg/cell or higher) than commonly reported for $\mathrm{CHO}$ cells (300-400 pg/cell) ${ }^{57,85,86}$. This can be explained by the larger cell size $(17 \mu \mathrm{m}$ diameter on day 4) of the $\mathrm{CHO}$ cell line used in this study, compared to those reported (12-14 $\mu$ m diameter) ${ }^{57}$. We applied the same DW measurement for another cell line with an average cell diameter of $14 \mu \mathrm{m}$ at a viability higher than $90 \%$, which 
resulted in $\sim 330 \mathrm{pg} / \mathrm{cell}$ (data not shown). Furthermore, the amino acid composition of the cellular protein and the fatty acids composition of the lipids analyzed in this study (ESM_3 Table S1 \& S2 of the online published data) are comparable to those reported for other $\mathrm{CHO}$ cells ${ }^{87,88}$. With respect to the content of protein, lipids, carbohydrates DNA, and RNA, such data could not be found in or derived from $\mathrm{CHO}$ cell studies and therefore, a comparison to the biomass composition of hybridoma cells ${ }^{83,84}$ is shown (Table I). As can be seen, the compositions are more or less comparable.

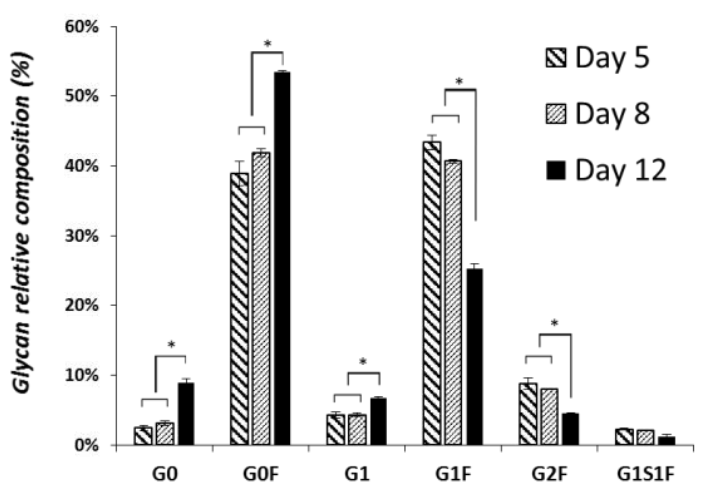

Figure 8. Relative mAb $\mathrm{N}$-glycan composition (\%) of the $10 \mathrm{~L}$ cultures. $\mathrm{N}$-glycans with different numbers of terminal residuals (G: galactose, $F$ : fucose, S: sialic acid) are shown. Error bars show the standard deviation of the triplicate runs. *: significantly different $(P<0.05)$

The results for the $\mathrm{CHO}$ cell line used in this study show that the mAb specific productivity is directly proportional to the cell volume (Figure 3a \& 3c), and further confirm that the $\mathrm{mAb}$ glycosylation profile does not change as the cell size increases (Figure 8). This is in agreement with Lloyd et al. ${ }^{56}$, who argued that the cell size is the major cellular determinant of productivity rather than the cell cycle phase. In addition to this, Edros et al. ${ }^{89}$ and Khoo and Al-Rubeai ${ }^{90}$ showed that the increase in specific productivity is directly related to the increase in cell volume due to increase in transcription, translation, and secretion machinery. Kim et al. ${ }^{58}$ reported that the enhancement of the specific thrombopoietin productivity is linearly correlated with the increase in cell size for nine $\mathrm{CHO}$ cell clones generated using the same transfection process. In a number of studies the relation between cell size and specific productivity was used to enhance $\mathrm{mAb}$ productivity, by directly or indirectly increasing the cell size, for example, through cell cycle arrest ${ }^{53,91}$, hyperosmotic pressure ${ }^{92,93}$, and mild hypothermia ${ }^{57,94}$.

Acetic acid and formic acid accumulation were observed during the cultures (Figure 2b). Possibly the acetic acid is formed from acetyl-CoA by one of the thioesterases that may be present in $\mathrm{CHO}$ cells ${ }^{95}$. This is further supported by the formation of isovaleric acid during the SI phase (Figure $\mathbf{2 b}$ ), which is formed from isovaleric acid$\mathrm{CoA}$ as a breakdown product of leucine ${ }^{96}$ and also requires the activity of an esterase. The formic acid concentration in this experiment reached $4 \mathrm{mM}$ on day 5 (Figure $\mathbf{2 b}$ ), 
which concentration was shown to inhibit $\mathrm{CHO}$ cell growth ${ }^{97}$. Formic acid is considered a breakdown product of serine ${ }^{71,98}$. In the present study, formic acid is mainly produced by the breakdown of serine through folate metabolism (ESM_1 of online published data), which is also reported by Carinhas et al. ${ }^{71}$ and Duarte et al..$^{98}$ and a small fraction is generated in the breakdown of tryptophan and during synthesis of cholesterol (ESM_1 of online published data). The high breakdown rates for some amino acids are likely caused by the overfeeding of these nutrients after the NI phase as can be seen in Figure 2. In addition, lactate concentration showed a peak at $30 \mathrm{mM}$ at around the point where the transition from the NI to SI phase occurred (Figure 2). However, the lactate concentration in this study is not expected to be the trigger of the phase transition, since the cell clone used in this study was observed to be able to grow in cell concentration at a lactate concentration up to $40 \mathrm{mM}$ (data not shown).

During the SI phase, the slightly higher fatty acids synthesis fluxes (Figure 7) occurred together with the formation of lipid droplets (Figure 6). Formation of lipid droplets has been reported in $\mathrm{CHO}$ cells ${ }^{99}$. In addition, high fatty acids synthesis rate during stationary phase was previously reported in a $\mathrm{CHO}$ cell study using ${ }^{13} \mathrm{C}$ isotopic tracers ${ }^{75}$. However, the fate of the excessive fatty acids was unclear in that study. We hypothesize that the accumulation of lipids in the present study is mainly caused by a maintained fatty acids synthesis rate in combination with a decreased membrane lipid demand during the SI phase. A slight increase in the fatty acid synthesis flux from the NI (0.07) to the SI (0.09) phase (Figure 7) is observed. The amount of extra $\mathrm{NAD}(\mathrm{P}) \mathrm{H}$ needed for this is negligibly small compared to the total $\mathrm{NAD}(\mathrm{P}) \mathrm{H}$ oxidation flux. The slight increase in the fatty acid synthesis flux, therefore, is not expected to largely affect the overall redox rearrangement and through this play a role in the change in lactate metabolism.

\subsubsection{Metabolic patterns of cell size increase}

Based on FVA, higher $\mathrm{O}_{2}$ consumption and oxidative phosphorylation fluxes were calculated in the SI phase as compared to the NI phase. This results in a higher ATP synthesis flux during the NI phase, which is reflected in the higher ATP translocation flux from mitochondria to cytosol in the SI phase (Figure 7). The higher flux through oxidative phosphorylation means that more NADH is generated in the SI phase. The $\mathrm{NADH}$ can be generated in glycolysis and the TCA cycle or by the combined activity of the PPP and transhydrogenase systems. However, the fluxes in these pathways cannot be independently calculated (shown by the red flux values in Figure 7) and thus it remains unknown whether the increased $\mathrm{O}_{2}$ consumption and oxidative phosphorylation activity are due to an increase in TCA flux or an increased flux through the PPP or both. Bi et al. ${ }^{52}$ reported a significant increase in mitochondrial activity (1.6-fold) and mitochondrial mass (twofold) for a $\mathrm{CHO}$ cell line with a 
similar cell volume increase (three- to fourfold). Furthermore, Zagari et al. ${ }^{100}$ showed that the lactate metabolism shift from production to consumption was correlated with higher mitochondrial activity. The same lactate metabolic shift also occurred in the SI phase of our study, which also may point to an increase in TCA cycle activity during the SI phase. These studies support that the increased NADH generation flux during the SI phase is mainly generated from a higher flux through the TCA cycle. However, Sengupta et al. $^{72}$ and Templeton et al. ${ }^{73}$ showed by using isotopic labeling that the flux through both the oxidative PPP as well as through the TCA cycle increased during the non-growth phase. In addition, Ahn and Antoniewicz ${ }^{60}$ reported a significant increase in oxidative PPP together with a constant TCA flux from the growth phase to the stationary phase. However, in these last three studies, the increase in cell size was not reported and thus the metabolic condition of the nongrowth phase in their studies may not be fully illustrative for the SI phase of this study. In conclusion, increased $\mathrm{O}_{2}$ consumption and oxidative phosphorylation fluxes are calculated and it seems most likely that an increased TCA cycle flux is the cause for the faster NADH generation in the SI phase.

The increased NADH demand and likely higher TCA cycle activity to generate this $\mathrm{NADH}$ could result in a lower NADH/NAD ratio and a lower pyruvate concentration in the cell. This might be related to the metabolic switch from lactate production to consumption, since the conversion of lactate to pyruvate generates $\mathrm{NADH}$ and the pyruvate that is generated can support the higher TCA flux. The higher flux through oxidative phosphorylation may have two causes: (i) a higher energy demand of cells in the SI phase for maintenance and/or growth. This may be directly related to cell size, but may also be related to other factors that change over time like for example the ammonium concentration. The energy requirement for maintenance can increase when cells are exposed to ammonium due to its competitive inward transport over potassium ions via the $\mathrm{Na}^{+} / \mathrm{K}^{+}$-ATPase and the $\mathrm{Na}^{+} \mathrm{K}^{+} 2 \mathrm{C1}^{-}$cotransporter ${ }^{101}$. However, the ammonium is not likely to cause an increase in energy demand since its concentration remains reasonably constant between the NI and SI phase. (ii). A decrease in mitochondrial efficiency by uncoupling of the oxidative phosphorylation. An "uncoupler" (i.e. leaky membrane) enables protons to flow back without passing through the ATP synthase, therefore, less ATP is generated from the same amount of $\mathrm{NADH}$ and $\mathrm{FADH}_{2}$ (i.e. a lower $\mathrm{P} / \mathrm{O}$ ratio) ${ }^{102}$. If the demand for ATP stays the same, a higher flux through oxidative phosphorylation and a higher $\mathrm{O}_{2}$ consumption rate are needed to meet this demand. The thioesterases responsible for the production of acetic acid and isovaleric acid as mentioned before may also be responsible for the release of free fatty acids that are known to uncouple the oxidative phosphorylation ${ }^{103,104}$. The slightly higher fatty acids synthesis fluxes during the SI phase could be related to this. 


\subsubsection{Implications of cell size increase during a culture process}

The results of this study stress that cell size and the amount of biomass per cell should be taken into account during the process characterization. Based on cell density measurement alone, the threefold increase in biomass observed in this work would not have been captured (Figure 1), and the cell size increase phase would have been mistakenly identified as part of the stationary phase. In fact, a two-micron increase in cell diameter, for instance, from 14 to $16 \mu \mathrm{m}$, may already lead to a 50\% increase in total cell volume or biomass. Furthermore, it can skew the process interpretation such as final biomass concentration reached, mAb productivity, and cellular metabolism. Similarly, skewed biological interpretation of a transcriptomic study due to cell size changes was reported by Fomina-Yadlin et al. ${ }^{31}$. The occurrence of cell size increase is also important for feed development. Since biomass continues to grow after the exponential cell concentration increase phase, the nutrients provided to the culture should contain not only nutrients assigned for maintenance and $\mathrm{mAb}$ production reactions, but also all the precursors and building blocks for biomass growth. In this specific culture process, a simple feeding concept would be to keep the feeding rate of essential amino acids at a per cell level the same during the SI phase and the NI phase, since the cell number-specific consumption rates remain fairly constant (Figure 5a).

The present study further shows that during the SI phase the average $\mathrm{mAb}$ production per cell increased by a factor 2 (Figure 3), while the average $\mathrm{O}_{2}$ consumption and $\mathrm{CO}_{2}$ production increased only by a factor 1.5 (Figure 7). In addition, compared to the NI phase, during the SI phase a higher proportion of the amino acids taken up from the medium is used for $\mathrm{mAb}$ synthesis (Figure 7). Collectively, the amount of mAb production per mol of oxygen and amino acid consumed increases for larger cells, which can be used for further process optimization. Especially for high-density perfusion processes where $\mathrm{O}_{2}$ and/or $\mathrm{CO}_{2}$ transfer may be limiting, it may be favorable to have larger cells.

This study demonstrates that cell size is an important factor for characterizing and optimizing a cell culture process. Correlations between the cell size increase and metabolic changes were found. However, further research is needed to understand the relationship between changes in cell size and changes in metabolism. In addition, studies on the cellular pathways that control cell size, such as the mammalian target of rapamycin (mTOR) pathway 105,106, and the extracellular stimuli involved could promote mechanistic understandings for the cell size increase, and enable controlled cell size manipulation during a culture process. 



\section{Chapter 4}

\section{Transcriptome analysis of $\mathrm{CHO}$ cell size increase during a fed-batch process}




\begin{abstract}
:
Transcriptome analysis is performed to identify the molecular mechanisms associated with a cell size increase phase observed in a $\mathrm{CHO}$ fed-batch process. Cell cycle analysis reveals that upon the stop of division, the cells are arrested in both the $\mathrm{G}_{0} / \mathrm{G}_{1}$ and $\mathrm{G}_{2} / \mathrm{M}$ phase. Moreover, in both phases, the cells continue to grow in size. Gene expression results show that the cell cycle arrest is associated with significant up-regulation of cyclin-dependent kinases inhibitors (CDKNs) and down-regulation of various cyclin-dependent kinases (CDKs) and cyclins that are involved in all cell cycle phases. During the cell size increase phase, the gene expression of the upstream pathways of mTOR, which are linked to the extracellular growth factor, cytokine, and amino acid conditions, shows a strongly synchronized pattern to promote the mTOR activity. In addition, the downstream genes of mTOR, such as 4ebp1 and Srebf1, also show a synchronized pattern to stimulate protein translation and lipid synthesis. The results demonstrate that a strong cell cycle inhibition with a stimulated mTOR activity at the transcriptome level are important elements for the observed $\mathrm{CHO}$ cell size increase. Furthermore, the activities of the cell cycle and mTOR regulator genes show a close link to the extracellular nutrient conditions through a number of cascade pathways. This indicates that by rational design of media and feeds, $\mathrm{CHO}$ cell size can be manipulated during industrial culture processes, which may further improve cell growth and specific productivity.
\end{abstract}

Xiao Pan ${ }^{a}$,

Abdulaziz A Alsayyari a,

Ciska Dalm ${ }^{\mathrm{b}}$,

Jos A. Hageman c,

René H. Wijffels a, ,

Dirk E. Martens ${ }^{a^{*}}$ a Bioprocess Engineering, Wageningen University \& Research, PO Box 16, 6700 AA, Wageningen, the Netherlands

b Upstream Process Development, Synthon Biopharmaceuticals BV, PO Box 7071, 6503 GN, Nijmegen, the Netherlands

${ }^{c}$ Biometris, Wageningen University \& Research, P.O. Box 16, 6700 AA, Wageningen, the Netherlands

d Faculty of Biosciences and Aquaculture, Nord University, N8049, Bodø, Norwav 


\section{Introduction}

Chinese Hamster Ovary $(\mathrm{CHO})$ cells are currently the predominant expression system for the production of biopharmaceuticals. In order to increase the volumetric productivity of $\mathrm{CHO}$ cell cultures, advances have been made with respect to media development, process optimization, and cell line engineering. To obtain an increased volumetric productivity without increasing cell density, an increase in specific productivity $\left(\mathrm{q}_{\mathrm{p}}\right)$ is needed. For this, several strategies have been described, including hyperosmotic pressure ${ }^{92}$, cell cycle arrest ${ }^{53}$, and mild hypothermia ${ }^{57}$. In these studies, the increase in $\mathrm{q}_{\mathrm{p}}$ was found to be correlated with an increase in cell size. Furthermore, Kim et al..$^{58}$ showed that the enhancement of the $\mathrm{q}_{\mathrm{p}}$ was linearly correlated with an increase in cell size for nine $\mathrm{CHO}$ cell clones generated using the same transfection process. Edros et al..$^{89}$ and Khoo and Al-Rubeai ${ }^{90}$ showed that the increase in $\mathrm{q}_{\mathrm{p}}$ was directly related to the increase in cell volume due to enhancement of the transcription, translation, and secretion machinery.

The regulation of mammalian cell size is controlled by cell growth and cell division through multiple complex mechanisms and pathways ${ }^{107,108}$. Proliferating cells are maintained at a more or less constant cell size by doubling their cell mass before each cell division. The mechanistic target of rapamycin (mTOR) signaling pathway has shown to be a central controller of cell size by controlling biomass synthesis ${ }^{109-111}$. Dreesen and Fussenegger ${ }^{112}$ showed that an increase in the mTOR activity led to an increase in $\mathrm{CHO}$ cell size together with an up to four-fold higher protein content per cell, and a four-fold higher specific productivity of recombinant IgG. Furthermore, manipulation of the positive or negative upstream pathways of mTOR has been shown to influence mammalian cell size. Backman et al. ${ }^{113}$ showed that deletion of a mTOR inhibitor, phosphatase and tensin homolog (PTEN), resulted in mammalian cell size increase. McVey et al. ${ }^{114}$ showed that the knock-out of another mTOR inhibitor, tuberous sclerosis complex 2 (TSC2), also resulted in larger $\mathrm{CHO}$ cells with higher protein synthesis and an over two-fold increased $\mathrm{q}_{\mathrm{p}}$. In addition, an increase in the PI3K/Akt pathway activity which positively regulates mTOR resulted in cell size increase in both mammals and Drosophila ${ }^{108}$.

As mentioned before, cell size is also altered during the cycle of each cell division. Cell size and biomass content per cell increase during the interphase $\left(\mathrm{G}_{1}, \mathrm{~S}\right.$, and $\mathrm{G}_{2}$ phase) of the cell cycle. A cell reaches the largest cell size at the end of the G2 phase, which is two times the size as the cell in the $\mathrm{G}_{1}$ phase, before the mitosis (M phase) takes place ${ }^{115}$. Hence, cell cycle control is also a way to increase cell size. In the study of Bi et al..$^{91}$, overexpression of the p21 ${ }^{\mathrm{CIP} 1}$ induced $\mathrm{G}_{1}$-phase cell cycle arrest in a $\mathrm{CHO}$ cell culture, which resulted in nearly fourfold increase in cell volume as well as in $\mathrm{q}_{\mathrm{p}}$. In the study of Fomina-Yadlin et al. ${ }^{31}$, the addition of small molecule inhibitors that arrest mammalian cells in the $\mathrm{G}_{1}$ phase or $\mathrm{G}_{1} / \mathrm{S}$ transition resulted in a 1.5-fold increase in cell volume and $\mathrm{q}_{\mathrm{p}}$. The regulation of cell size is a complex process that 
involves a large network including both cell growth and division. Until now, the molecular mechanism of cell size regulation is not fully understood.

In Chapter $3^{116}$, a cell number increase (NI) phase followed by a cell size increase (SI) phase was observed in a $\mathrm{CHO}$ cell fed-batch culture process using ActiCHO-P as basal medium and Actifeed A/B as feeds (from now on called the ActiCHO process). In the cell size increase phase, cell division comes to a halt but cell growth continues in the form of an increase in cell size. In this process, a standard feeding approach was used. No special strategies, such as lowering of the temperature, were used to arrest the cell cycle. The increase in cell volume was due to an increase in cell dry weight as a result of increases in protein, lipid, and carbohydrate content per cell. Furthermore, an increase in $\mathrm{q}_{p}$ was observed, which linearly correlated with the increase in cell volume. The metabolic changes associated with the cell size increase were studied in detail using flux balance analysis, however, the cause and molecular mechanisms for the cell size increase remained unclear. The aim of the present study is to obtain more insights into the cause and the molecular mechanisms involved using transcriptome analysis.

Due to the dynamic nature of the fed-batch culture (e.g. continuously changing cell density and nutrient and waste metabolite concentrations), many genes will be regulated over time of which only a part is related to the cell size increase. In order to enrich the genes that are related to cell size regulation, we used a FortiCHO process (FortiCHO basal medium fed with Efficient feed A/B) which did not result in cell size increase as a "filter". In this way, the genes that are significantly regulated as a result of culture development but are not related to cell size increase can be identified and filtered out.

\section{Materials and methods}

\subsection{Cell line and fed-batch process set-up}

A suspension $\mathrm{CHO}^{\mathrm{BC}}$ cell clone (BC-P, provided by Bioceros Holding BV) producing a recombinant immunoglobulin G1 (IgG1) as described in Chapter $2^{8}$ was used in this study. Two fed-batch processes using the same cell line but different mediumfeed systems are studied. One process resulted in cell size increase whereas for the other this was not the case.

(i) The ActiCHO culture process. Triplicate fed-batch cultures were conducted in $10 \mathrm{~L}$ Sartorius bioreactors (sartorius stedim) controlled by BIOSTAT ${ }^{\circledR}$ B-DCU II. Each bioreactor was inoculated with a starting density of $3 \times 10^{5}$ viable cells $/ \mathrm{mL}$ at a starting volume of $5 \mathrm{~L}$. Culture temperature was controlled at $37^{\circ} \mathrm{C}$, dissolved oxygen (DO) was controlled at $40 \%$ by pure $\mathrm{O}_{2}$ flow enrichment, $\mathrm{pH}$ was controlled at 7.2 by $\mathrm{CO}_{2}$ flow enrichment and $0.5 \mathrm{M} \mathrm{NaOH}$. The feeding strategy was the same as described in 
Chapter $3^{116}$. Briefly, from day 3 on, $4.5 \%$ ActiCHO feed A and $0.45 \%$ ActiCHO feed $\mathrm{B}$ (both from GE Healthcare) per culture volume per day were fed to each reactor daily. A $45 \%(\mathrm{w} / \mathrm{w})$ glucose solution was added to each bioreactor, to ensure that the glucose concentration stayed above $5 \mathrm{mM}$ at the next feeding point.

(ii) The FortiCHO culture process. Triplicate fed-batch cultures were conducted in 1 L DASGIP bioreactors (Eppendorf). Each bioreactor was inoculated with a starting density of $3 \times 10^{5}$ viable cells $/ \mathrm{mL}$ at a starting volume of $500 \mathrm{~mL}$. Culture temperature, $\mathrm{DO}$, and $\mathrm{pH}$ are controlled at the same set-point as the ActiCHO process with similar approaches. From day 3 on, Efficient Feed A/B ${ }^{\text {TM }}$ (with a 1:1 combination) was added to each bioreactor as one bolus. The feeding strategy was similar as described in Chapter $2^{8}$. Briefly, the daily volume added was calculated based on the specific glucose consumption rate, to ensure that the glucose concentration stayed above 5 $\mathrm{mM}$ at the next feeding point.

\subsection{Daily sampling and analysis}

A sample was taken from each bioreactor every day before the feed addition. Viable cell density, viability, and cell diameter were measured using a CedexHiRes ${ }^{\circledR}$ analyzer (Innovatis; Roche) for the ActiCHO process, and a TC20 ${ }^{\mathrm{TM}}$ cell counter (Bio$\mathrm{Rad}$ ) for the FortiCHO process. $3 \mathrm{~mL}$ of the sample was spun down at $300 \times \mathrm{g}$ for 5 min (Heraeus Multifuge X3R, Thermo Scientific). The cell pellet was collected and the total RNA was extracted using $3 \mathrm{~mL}$ TRIzol reagent (Invitrogen ${ }^{\mathrm{TM}}$ ) and stored at $80^{\circ} \mathrm{C}$ for later transcriptome analysis. For cell cycle analysis, $2 \mathrm{~mL}$ cell culture sample was spun down at $300 \times \mathrm{g}$ for $5 \mathrm{~min}$. The cell pellet was fixated in $2 \mathrm{~mL}$ ice-cold $70 \%$ ethanol overnight at $-20^{\circ} \mathrm{C}$. Next, the cells were prepared and stained using Cycletest $^{\mathrm{TM}}$ Plus DNA Reagent Kit (BD Biosciences) and the cell cycle was analyzed on the BD Accuri ${ }^{\mathrm{TM}}$ C6 Flow Cytometer (BD Biosciences).

\subsection{Transcriptome analysis}

For each bioreactor of the ActiCHO and FortiCHO process, transcriptome analysis was done for the samples taken on culture day 3, 5, and 7. Total RNA was purified using the RNeasy Mini kit (Qiagen, Valencia, CA). RNA concentration was measured on a Nanodrop 2000 (Thermo Scientific, Wilmington, DE). RNA quality was assessed on a 2100 Bioanalyzer using the RNA 6000 Nano Kit (Agilent, Santa Clara, CA), and all samples had a greater than 8 in RNA integrity number (RIN). Affymetrix GeneChip $^{\text {TM }}$ CHO Gene 2.1 ST Arrays (Affymetrix, Santa Clara, USA) were used for transcriptome expression profiling. In short, the same amount of RNA was labeled by the Whole-transcript Sense Target Assay (Affymetrix, Santa Clara, USA) and hybridized according to the manufacturer's instructions. Quality control and data analysis were done as described in Lin, K. et al. ${ }^{117}$. Normalized expression estimates 
of probe sets were computed by the robust multi-array analysis (RMA) ${ }^{118}$, using $\mathrm{R} /$ Bioconductor package AffyPLM ${ }^{119}$. The well-annotated reference sequences are based on the CriGri_1.0 genome assembly NCBI reference sequence project (RefSeq) Release 72, which resulted in 60626 annotated sequences (transcripts profiles) (custom CDF v20). After averaging the expression levels of probe sets targeting the same gene, expression data for 20858 unique genes were obtained, which was used for all subsequent analysis. Linear models (Limma package) were used to identify the differential expressed genes taking into account correlation due to the repeated sampling from the same bioreactors followed by an intensity-based moderated $t$ statistic $^{120,121}$. P-values were adjusted for multiple testing using the Benjamin and Hochberg (FDR.BH) method ${ }^{122}$. Genes regulation that satisfied the criterion of FDR.BH $<0.05$ and absolute fold-change $(F C)>1.4$ were defined to be significant and were subjected to KEGG pathways over-representation analysis using fisher's exact test and Venn diagram. These cut-off values are commonly applied to reduce the number of false-positive genes and the background noises that are picked up by the differential gene analysis ${ }^{51,123,124}$.

Gene regulations that satisfied the criterion of Benjamin and Hochberg false discovery rate $(\mathrm{FDR} . \mathrm{BH})<5 \%$ and absolute fold-change $(\mathrm{FC})>1.4$ were defined to be significant and were subjected to KEGG pathways over-representation analysis using fisher's exact test. These cut-off values are applied to reduce the number of falsepositive genes and the background noises that are picked up by the differential gene analysis ${ }^{51,123,125}$.

\section{Results and Discussion}

\subsection{Cell growth, cell size, and cell cycle dynamics}

The cell growth characteristics including viable cell density (VCD), viability, and average cell diameter are shown in Figure 1. The VCD profile is similar for both processes (Figure 1A). The cell viability in the ActiCHO process (black dashed line) started to decrease on day 10, while in the FortiCHO process (red dashed line) the decrease started on day 7. Figure 1B shows that for the ActiCHO process, upon the stop of cell division on day 5 , the cells continued to grow in size, from an average cell diameter of $17 \mu \mathrm{m}$ on day 4 , to $24 \mu \mathrm{m}$ on day 10. The ActiCHO process in Chapter $3^{116}$ showed that the size increase was proportional to the increase in biomass content. In contrast, the average cell diameter in the FortiCHO process went down gradually from $17 \mu \mathrm{m}$ on day 1 , to $15 \mu \mathrm{m}$ day 7 . The sharp decrease in cell diameter after day 7 was caused by cell death resulting in the formation of small particles which were probably apoptotic bodies. 

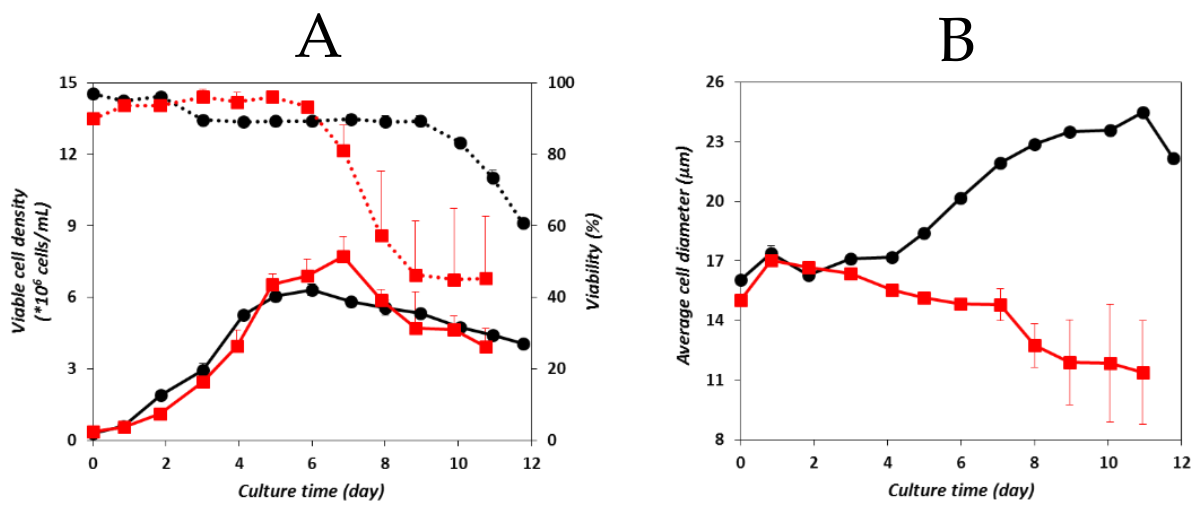

Figure 1. (A) Viable cell density $\left(\times 10^{6} \mathrm{cells} / \mathrm{mL}\right.$, solid lines) and viability (\%, dashed lines), and (B) average cell diameter $(\mu \mathrm{m})$ of the ActiCHO process in Chapter $3^{116}$ (round mark, black lines) and the FortiCHO process (square mark, red lines). The error bars show the standard deviation for three bioreactors of each process. In (A), only the positive error bars are shown in order to avoid overlap.

To study the cell cycle status during the cell size increase of the ActiCHO process, the cell cycle distribution was measured. In Figure 2, the propidium iodide (PI) DNA stain intensity area (FL2-A) which is a measure of the DNA content, is plotted against the forward scatter area (FSC-A) which is a measure of cell size for day 3, 5, 7, and 9. A clear cell cycle distribution can be seen at FL2-A intensities between 10 and 2.000.000. The populations observed above a FL2-A of 2.000.000 are probably due to aggregates. On day 3 they represent about 3\% of the total events and they gradually disappear with the progression of culture time to about $1.5 \%$ on day 9 . With culture time a population develops at very low FL2-A intensities which probably represents cell debris and apoptotic bodies and agrees with the slight decrease in viability observed. It can be seen from Figure 2 that:

(i) The cells in the $\mathrm{G}_{2} / \mathrm{M}$ phase are larger (higher FSC-A) than the cells in the $\mathrm{G}_{0} / \mathrm{G}_{1}$ phase for all the analyzed culture days. This agrees with the fact that the volume and biomass per cell increase from the $\mathrm{G}_{1}$ phase until the $\mathrm{G}_{2} / \mathrm{M}_{\text {phase }}{ }^{126}$.

(ii) Going from day 3 to day 9, it can be seen that the S-phase population disappears and cells are arrested in both the $\mathrm{G}_{0} / \mathrm{G}_{1}$ and the $\mathrm{G}_{2} / \mathrm{M}$ phase. When the cell debris population is gated out from the total population, the $\mathrm{G}_{0} / \mathrm{G}_{1}$ population presents $58 \%$ of the total population on day 3 and increases to $85 \%$ on day 9 , whereas for the $G_{2} / M$ population the number decreases from $31 \%$ on day 3 to $11 \%$ on day 9 (data not shown). The result agrees with the fact that after day 5 cell proliferation has stopped in the ActiCHO process (Figure 1). In several $\mathrm{CHO}$ cell culture processes, hypothermia ${ }^{19,127,128}$, hyperosmotic stress ${ }^{92,129}$, and cell cycle inhibitors ${ }^{53,91,130}$ were applied that mediated a $\mathrm{G}_{0} / \mathrm{G}_{1}$ arrest without influencing $\mathrm{G}_{2} / \mathrm{M}$ phase. This was, however, not the case in the present study where cell cycle arrest occurred in both $\mathrm{G}_{0} / \mathrm{G}_{1}$ and the $\mathrm{G}_{2} / \mathrm{M}$ phase. In theory, cells at twice the FL2-A intensity could be aggregates of two cells arrested in the G1phase. 
(iii) Cell size starts to increase after day 5 in both the $\mathrm{G}_{0} / \mathrm{G}_{1}$ and $\mathrm{G}_{2} / \mathrm{M}$ phase, as shown by the increase in FSC-A values. The extent of the size increase in both phases is comparable. This indicates that the cell size increase is not influenced by the cell cycle phase the cell is arrested in. Rather, the cell biomass growth and cell cycle arrest seem to be two independent processes.

In summary, the cell cycle analysis confirms that the cell cycle arrest occurs in both the $\mathrm{G}_{0} / \mathrm{G}_{1}$ and the $\mathrm{G}_{2} / \mathrm{M}$ phase of the ActiCHO process. Moreover, the cells arrested in both phases are able to grow in cell size.

A. Day 3

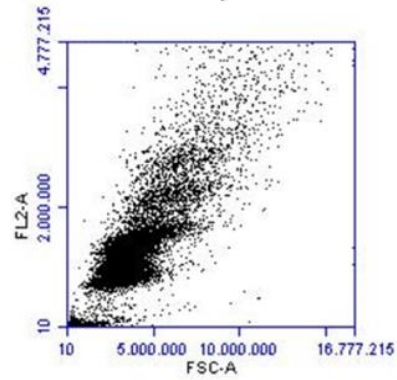

C. Day 7

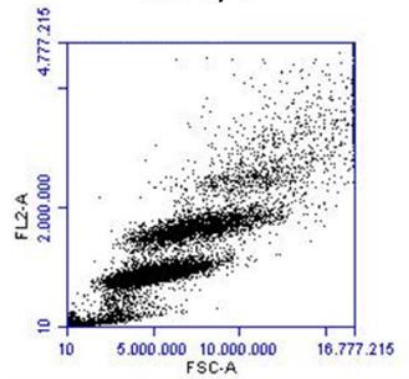

B. Day 5

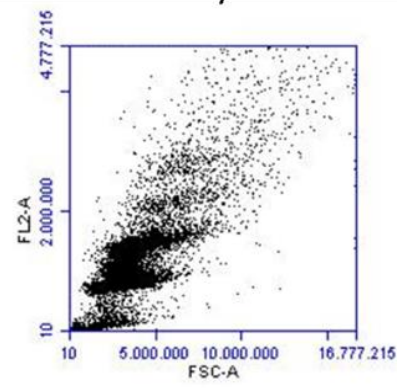

D. Day 9

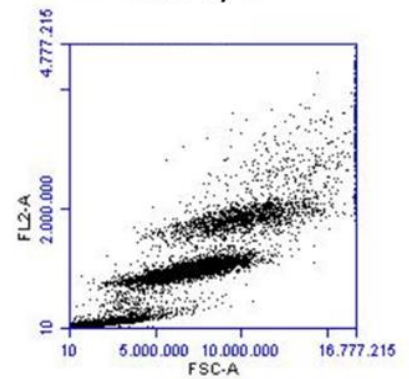

Figure 2. Cell cycle analysis for the ActiCHO process on day $3(A), 5(B), 7(C)$, and $9(D)$. The propidium iodide (PI) DNA stain intensity (FL2-A) is plotted against the forward scatter area (FSC-A). FL2-A indicates the amount of DNA in a cell while FSC-A indicates the size of a cell. The sample events at high FL2-A intensity (>2.000.000) are likely caused by cell aggregates.

\subsection{Transcriptome analysis}

Transcriptome analysis using Affymetrix $\mathrm{CHO}$ Gene microarrays was performed to gain insights into the molecular mechanism underlying the cell cycle arrest and the cell size increase. The same amount of mRNA was brought on $\mathrm{CHO}$ Gene microarrays for each sample during the transcriptome analysis. Therefore, a change in gene expression is relative against the total transcripts and is not influenced by the possible fact that larger cells have a higher overall transcriptome activity.

The sample points on culture day 3,5, and 7 of the two processes are selected for transcriptome analysis. Day 3 represents the middle of the cell proliferation phase where the cell size is still similar in both processes (Figure 1). For the ActiCHO process, day 5 represents the start of the cell size increase phase whereas day 7 
represents the middle of the cell size increase phase. For the FortiCHO process, day 5 represents the stop of exponential growth phase whereas day 7 represents the end of the stationary phase and the start of the death phase.

\subsubsection{Overall transcriptome profiles}

The principal component analysis (PCA) (Figure 3A) shows the overall variation in gene expression for all analyzed samples. PC1 and PC2 contribute $26 \%$ and $18 \%$ to the total variation, respectively. The contribution for the rest of the PCs are minor (e.g. $7 \%$ of the PC3) and are not considered. PC1and PC2 both capture a change in gene expression in time as well as a difference in gene expression between both systems (ActiCHO and FortiCHO). The Venn diagram (Figure 3B) shows the differential gene expression between day 3 and 7 which is unique to the ActiCHO and FortiCHO process, and which occurs in both processes. Genes are considered differentially expressed between the two days if the FDR.BH value is smaller than $5 \%$ and the absolute fold change is larger than 1.4 (see section 2.3). For the total 20858 probed genes on the $\mathrm{CHO}$ gene array, 5684 genes $(25 \%)$ are regulated in either the ActiCHO or FortiCHO process. In the ActiCHO process, 4824 genes (total number of genes in the blue circle) are differentially expressed, of which 3037 (total number of genes in the blue circle excluding the number of genes that overlap with the red circle) are unique to the ActiCHO process, and of which 1787 (overlap between the two circles) are also differentially expressed in the FortiCHO process in the same direction.

A

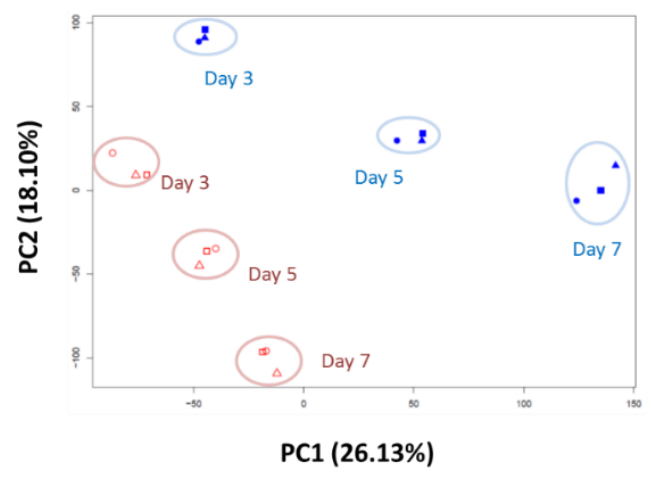

B

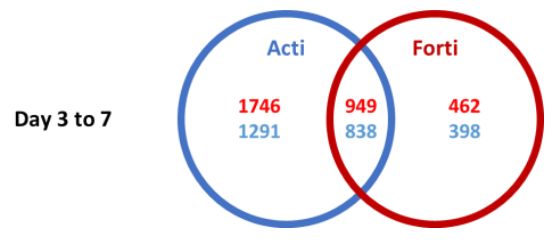

Figure 3. (A) Score plot generated from the transcriptome results of the ActiCHO (filled blue markers) and the FortiCHO (open red markers) processes. The same sample marker represents the sample taken from the same bioreactor. Samples taken from the same day and the same process is marked in a circle. (B) Venn diagram of the number of genes that are differentially regulated from day 3 to 7 compared between the two processes. Red numbers indicate up-regulation, blue numbers indicate down-regulation.

From the significantly regulated genes in the ActiCHO process from day 3 to 7 , we first selected the top 10 fold-changes of the up- and down-regulated individuals (Table I). Compared to the top 10 up- and down-regulated genes in the ActiCHO 
process, many of these genes also showed significant (fold-change $>1.4$, FDR.BH $<5 \%$ ) regulations in the FortiCHO process, however, the fold-changes are much smaller (data to be published in Supplementary 1). In the top 10 up-regulated genes in the ActiCHO process, the Fam $213 a$ and Ranbp3l genes have extremely high fold-changes (188 and 145, respectively) compared to the others. The expression of Fam213a was shown to protect cells from oxidative stress ${ }^{131}$. Oxidative stress in cells can be caused by increased reactive oxygen species (ROS) which are reduced forms of oxygen, such as superoxide radical $\left(\mathrm{O}_{2}^{-}\right)$, hydrogen peroxide $\left(\mathrm{H}_{2} \mathrm{O}_{2}\right)$, and hydroxyl radical $\left(\mathrm{HO}^{-}\right)$. The dissolved oxygen tension (DO) during all the cultures in this study was maintained at $40 \%$. It is unlikely that a high extracellular DO was the cause of the ROS formation. However, increased oxidative metabolism was reported in CHO cells with increased $\mathrm{mAb}$ production as well as with increased cell size ${ }^{52,73}$. In Chapter $\mathbf{3}^{116}$, a 1.5-fold increase in cellular oxygen consumption flux was observed during the size increase phase of the ActiCHO process. This might be related to the formation of ROS in cells and subsequently the overexpression of Fam213a. If this was the case, the extreme overexpression of Fam $213 a$ would likely be a response of the cell size increase, rather than the cause of it. Another extremely up-regulated gene, Ranbp3l, was reported to be involved in Bone Morphogenetic Protein (BMP) signaling and regulating mesenchymal stem cell differentiation ${ }^{132}$. The exact function of this gene in the context of cell size increase in CHO cells is however unknown. In addition, two genes involved in osmotic regulation (Slc6a12 and Scn7a) are significantly upregulated. This could be related to the increase in culture osmolality due to the feed addition which was described in Chapter 3 for the ActiCHO process ${ }^{116}$. Next to the top 10 up-regulated genes, also the top 10 down-regulated genes are listed in Table I, together with the functions of these genes in biological processes. As can be seen, all the top 10 down-regulated genes are involved in cell cycle regulation and mitosis. This agrees with the inhibition of cell cycle progression as described in the previous section. Some of these genes are further discussed in the next section.

Table I. Top 10 fold-changes of up- and down-regulation genes in the ActiCHO process from day 3 to 7. The gene functions are searched against the Gene Ontology $(G O)$ and UniProt database.

\begin{tabular}{ccc}
\hline & \multicolumn{2}{c}{ Top 10 up-regulation } \\
Gene symbol & Fold-change & Gene function in biological process \\
\hline Fam $213 a$ & 188.20 & Redox regulation \\
Ranbp3l & 145.63 & Protein transport \\
Sema4d & 24.12 & Differentiation \\
$S p p 1$ & 20.56 & Cytokine activity, extracellular matrix binding \\
$S l c 6 a 12$ & 19.64 & Osmotic regulation/transport \\
$M y 19$ & 14.68 & Myosin regulation \\
$P c p 4 l 1$ & 14.02 & Protein coding \\
$S c n 7 a$ & 13.78 & Sodium channeling
\end{tabular}




\begin{tabular}{ccc} 
S100a4 & 11.42 & $\begin{array}{c}\text { Protein binding } \\
\text { Protein coding }\end{array}$ \\
LOC100768899 & 11.40 & Top 10 down-regulation \\
Gene symbol & Fold-change & Mitosis, spindle assembly \\
\hline Kif15 & -17.19 & DNA synthesis, cell cycle \\
Ect2 & -16.84 & Mitosis, chromosome segregation \\
Nuf2 & -16.73 & DNA synthesis, cell cycle \\
Rrm2 & -16.55 & nucleosome assembly \\
LOC100752904 & -15.90 & Cell cycle \\
Ccna2 & -14.97 & Mitosis, chromosome segregation \\
Bub1b & -14.87 & Mitosis, chromosome segregation \\
Cenpw & -14.77 & Cell cycle \\
Ccnb2 & -14.63 & Mitosis, chromosome segregation \\
Aurkb & -14.08 &
\end{tabular}

In order to get a better understanding of the relationship between gene regulation and the cell size increase, a targeted approach is used in the next sections. From the genes that are differentially expressed in the ActiCHO process (the blue circle in Figure 3B), a subset of genes ( $\sim 500$ genes in total) are examined in more detail. This subset of genes are selected based on the fact that they are known to play critical roles in cell cycle progression and biomass growth as well as in the signaling pathways involved in these processes. The regulation of these genes is compared to that in the FortiCHO process, to enrich genes that are uniquely related to the cell size increase. An overview of the selected pathways is shown in Figure 4. Note that some pathways in Figure 4 are known to be post-transcriptional regulated (e.g. by phosphorylation), and it is not completely clear whether they are regulated transcriptionally as well. Thus, genes that are not differentially expressed could still be important due to post-transcriptional regulation. However, if the transcription of these pathways shows a synchronized regulation, it may still mean that at least part of the regulation is done transcriptionally, and it may still give an indication of these gene functions in cell size increase. 


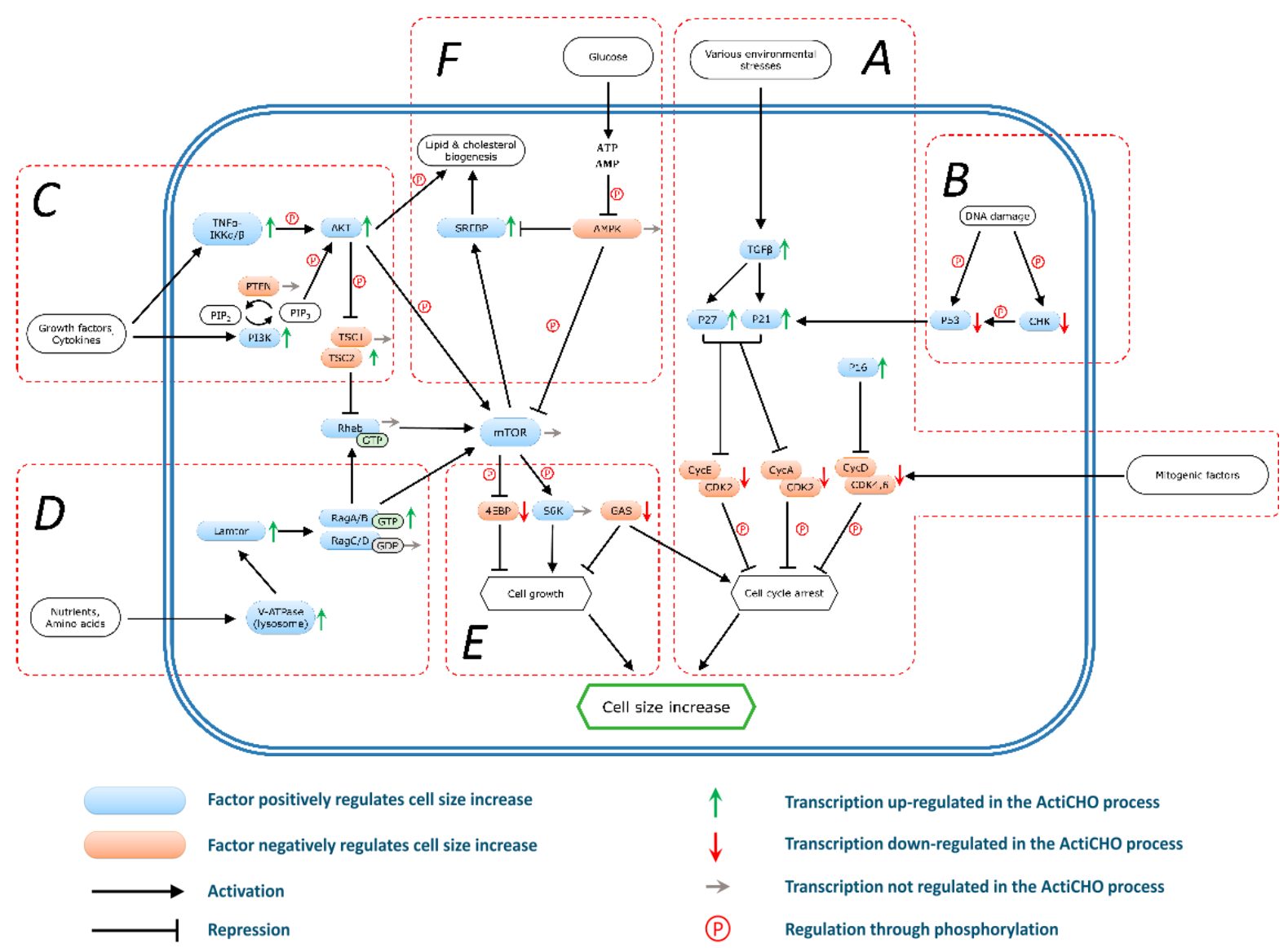

Figure 4. Signaling pathways channel the information of extracellular conditions to the cell cycle and cell growth regulations eventually result in cell size increase. Mammalian cell cycle progression is controlled by cyclin dependent kinases (Cdks) and their binding partner cyclins (Cycs). Cell cycle arrest can be achieved by intracellular signals initiated by DNA damage (Box A), and extracellular signals mediated by regulating factors such as transforming growth factor betas (TGFßs) (Box B). In the case of DNA damage, activities of various Cdk/Cyc complexes are inhibited through p53 and checkpoint kinases (CHK) pathways. In the case of extracellular stresses, $p 21^{C I P 1}$ and $p 27^{K I P 1}$ as inhibitors of Cdks can be transcriptionally induced by TGF $\beta$ s. The activities of Cdk/Cyc complexes are negatively regulated by a family of cyclin-dependent kinase inhibitors including $p 16^{I N K A a}, p 21^{C I P 1}$, and $p 27^{K I P 1}$. The Cdk4,6 and CycD complex also works as a sensor for extracellular mitogenic factors, which controls the progression of cell cycle. mTOR promotes the cell growth by activating translation and transcription through the regulation of its downstream factors such as P70-S6 Kinase 1 (S6K) and eukaryotic translation initiation factor $4 E$ (EIF4E) and its binding protein (4EBP) (Box E). $m$ TOR also promotes the lipid synthesis by regulating sterol responsive element binding protein (SREBP) activity (Box F). Protein kinase B (PKB/Akt) pathway can activate mTOR through tuberous sclerosis complex

(TSC) 1/2 dependent and independent pathways ${ }^{110}$ (Box C). The activation of Akt inactivates TSC1/2, promoting the GTP-bound form of Ras homolog enriched in brain (Rheb) protein. The latter one acts as an activated switch for the mTOR pathway. Akt can also promote the phosphorylation and dissociation of PRAS40 from mTOR complex 1 (mTORC1) thus release the mTOR activity through a TSC1/2 independent pathway ${ }^{133}$. Akt induces lipid synthesis by up-regulating the ATP citrate lyase (Acly) activity which is a key enzyme that catalyzes the reaction from citrate to cytosolic acetyl-CoA ${ }^{134}$ (Box C-F). Akt can be up-regulated upon sensing the extracellular growth factors (e.g. insulin, IGF, and TNFa) through the phosphatidylinositol 3'-kinase (PI3K)-Akt dependent pathway, as well as the TNFa-IKKa/ß mediated pathway ${ }^{135}$ (Box C). Upon sensing the extracellular amino acids, vacuolar $H^{+}$-ATPase (V-ATPase) is stimulated which activates the $m$ TOR regulator (Lamtor) (Box D). The regulator acts as a guanine exchange factor (GEF) that promotes the conversion of RagA/B-GDP to RagA/B-GTP which activates the mTORC1. A high ATP/AMP ratio can activate mTORC1 by preventing the activation of AMP-activated protein kinase (AMPK). The inactivation of

AMPK activity also favors the SREBP activity ${ }^{136}$ (Box F).The expression of growth arrest specific (GAS) genes are closely involved in growth suppression. They negatively regulate cell growth and cell cycle progression (Box E). 


\subsubsection{Cell cycle regulation}

Figure 4 Box $\mathbf{A}$ and $\mathbf{B}$ show the pathways related to the cell cycle regulation. The genes of these pathways that are significantly regulated from culture day 3 to 7 in the ActiCHO process are presented and their changes in expression are compared to those in the FortiCHO process (Table II). Mammalian cell cycle regulation involves the participation of two classes of proteins: the cyclin-dependent kinases (Cdks), and their binding partners, cyclins (Cycs) ${ }^{137}$. Binding of a Cdk to a Cyc forms an active complex that enables the cell cycle to progress through a specific phase. For both processes in Table II, down-regulations of various cyclin-dependent kinase genes $(C d k s)$ and their binding partner cyclin genes $(C y c s)$ are observed, which agrees with the stop of cell division in both processes. However, the extent of down-regulation (fold change) and significance (FDR.BH) for these genes are much higher in the ActiCHO process than in the FortiCHO process, indicating that the cell cycle arrest is stronger in the ActiCHO process.

For the ActiCHO process, especially one CycA gene (Ccna2, FC=-14.97, FDR.BH<0.5\%), two $\mathrm{CycB}$ genes (Ccnb1, FC=-11.20, FDR. $\mathrm{BH}<0.5 \%$, and $\mathrm{Ccnb2}$, FC=14.63, FDR.BH<0.5\%), and to a lesser extent a Cdk gene (Cdk1, FC=-3.89, FDR. $\mathrm{BH}<0.5 \%$ ), have much stronger down-regulations as compared to the other Cdk and Cyc genes (Table II). Binding of CycA with $\mathrm{Cdk}_{1}$ is required for the $\mathrm{G}_{2} / \mathrm{M}$ transition ${ }^{115}$, binding of CycA with Cdk2 plays an important role in $S$ phase progression and DNA replication ${ }^{138}$, and binding of $\mathrm{CycB}$ with $\mathrm{Cdk} 1$ is required for mitosis $^{115}$. The strong down-regulation of $\mathrm{CycA}, \mathrm{CycB}$ and $\mathrm{Cdk} 1$ genes in the ActiCHO process agrees with the observed arrest of the cell cycle in $\mathrm{G}_{2} / \mathrm{M}$ phase and indicates a delay in $S$ phase and mitosis. In addition, binding CycE with Cdk2 is required for progression through the $\mathrm{G}_{1} / \mathrm{S}$ phase ${ }^{139}$. The down-regulation of $C d k 2$ $\left(\mathrm{FC}=-2.29\right.$, FDR.BH<0.5\%) may be related to the cell cycle arrest in the $\mathrm{G}_{1}$ phase.

In order to find the cause of the cell cycle arrest, next, signaling pathways that coordinate the cell cycle are studied. The cell cycle activities are regulated by a number of Cdk inhibitors (Cdkns) such as p16 ${ }^{\mathrm{INK} 4 a}(C d k n 2 a), \mathrm{p}^{21^{\mathrm{CIP} 1}}$ (Cdkn1a), and p27KIP1 $(C d k n 1 b)^{115}$ (Figure 4 Box A). Binding of these Cdkns to the Cdks inhibits formation of the active $\mathrm{Cdk} / \mathrm{Cyc}$ complexes, which suppresses the cell cycle progression $^{137}$. The high up-regulations of $C d k n s$ ( $C d k n 2 a, \mathrm{FC}=1.62, \mathrm{FDR} . \mathrm{BH}<0.5 \%$, and $C d k n 1 a, \mathrm{FC}=1.73$, FDR.BH $<0.5 \%$ ) in the ActiCHO process indicate a strong cell cycle inhibition (Table II). In contrast, the Cdkns in the FortiCHO process showed much less regulation ( $C d k n 2 a, \mathrm{FC}=1.11, \mathrm{FDR} . \mathrm{BH}=24 \%$, and $\mathrm{Cdkn} 1 a, \mathrm{FC}=1.28$, FDR.BH<0.5\%).

Cell cycle inhibitors can be activated by intracellular stimuli (e.g. DNA damage), or extracellular stimuli (e.g. deprivation of mitogenic factors) $)^{140}$. The regulation pathways related to intracellular DNA damage are shown in Figure 4 Box B. Upon sensing DNA damage, checkpoint kinases (encoded by Chek1 and Chek2) and the p53 
tumor suppressor protein (encoded by Tp53) can be phosphorylated and activated, resulting in the $\mathrm{G}_{1}$ and $\mathrm{G}_{2}$ cell cycle inhibition ${ }^{141}$. Although these steps are regulated via post-transcriptional phosphorylation, the significant down-regulations of Chek1, Chek2, and Tp53 genes in both of the two processes (Table II) imply that DNA damage is less likely the cause of the cell cycle arrest. With regard to extracellular stimuli, the $\mathrm{p} 21^{\mathrm{CIP} 1}$ can be induced by transforming growth factor beta (TGF $\beta$, encoded by $T g f b$ ), which acts as a negative paracrine/autocrine growth factor in growth regulation ${ }^{142-144}$, as shown in Figure 4 Box A. The TGF $\beta$ protein is activated from its latent complex upon sensing various environmental stresses such as reactive oxygen species ${ }^{145}, \mathrm{pH}^{146}$, and proteases ${ }^{147}$. The TGF $\beta$ signaling pathway is initiated by binding of TGF $\beta$ to TGF $\beta$ receptors (type I and II encoded by Tgfbr1 and Tgfbr2) and subsequently, induces $\mathrm{p}^{\mathrm{KIP} 1}$ and $\mathrm{p} 21^{\mathrm{CIP} 1}$ leading to the cell cycle inhibition (Figure 4 Box A). The higher up-regulations of both the $T g f b$ (Tgfb3, FC=2.13, FDR.BH<0.5\%) and Tgfbr $($ Tgfbr2, FC=1.61, FDR.BH<0.5\%) genes in the ActiCHO process (Table II) indicate a higher TGF $\beta$ signaling activity. In addition, expression of $\mathrm{CycD}$ is strongly dependent on extracellular mitogens. The $\mathrm{CycD} / \mathrm{CDK} 4,6$ complexes, therefore, function early in $\mathrm{G}_{1}$ and act as mitogen sensors (Figure 4 Box A) ${ }^{148}$. The $\mathrm{CycD}$ genes (ccnd1, ccnd2, and ccnd3) are not regulated in the ActiCHO process (data to be published in Supplementary 1), indicating that deprivation of extracellular mitogens is not likely the cause of cell cycle arrest in the $\mathrm{G}_{1}$ phase. However, looking at the expression of various mitogen-activated protein kinases, we find both significant upand down-regulations in the ActiCHO process. Therefore, it is not certain whether lacking of mitogen is the cause of the cell cycle arrest in the phases other than $\mathrm{G}_{1}$. In summary, the result from transcriptome analysis implies that the cell cycle arrest in the ActiCHO process was caused by an activation of cell cycle inhibitors and downregulation of Cdks and cyclins in reaction to extracellular environmental stresses and/or depletion of mitogen(s).

Table II. Fold change (FC) cell cycle related genes from culture day 3 to 7 in the culture with cell size increase (ActiCHO) and with no cell size increase (FortiCHO). Positive values mean up-regulation and negative values mean down-regulation. Genes with a FDR.BH $<5 \%$ and the absolute FC $>1.4$ are considered significant and are shown here. (Arrest) means the genes promote cell cycle arrest whereas (promote) means the genes promote cell cycle progression.

\begin{tabular}{|c|c|c|c|c|}
\hline & FC. ActiCHO & FDR.BH & FC. FortiCHO & FDR.BH \\
\hline \multicolumn{5}{|c|}{ Cyclin-dependent kinase (Cdk) (promote) } \\
\hline$C d k 1$ & -3.89 & $<0.5 \%$ & -1.17 & $9 \%$ \\
\hline$C d k 2$ & -2.29 & $<0.5 \%$ & -1.76 & $<0.5 \%$ \\
\hline$C d k 4$ & -2.19 & $<0.5 \%$ & -1.36 & $<0.5 \%$ \\
\hline$C d k 5$ & 1.47 & $<0.5 \%$ & -1.03 & $81 \%$ \\
\hline$C d k 6$ & -1.74 & $<0.5 \%$ & -1.02 & $79 \%$ \\
\hline$C d k 15$ & 1.55 & $1 \%$ & 2.21 & $<0.5 \%$ \\
\hline Cyclin (C & omote) & & & \\
\hline
\end{tabular}




\begin{tabular}{|c|c|c|c|c|}
\hline Cona2 & -14.97 & $<0.5 \%$ & -1.77 & $<0.5 \%$ \\
\hline Ccnb2 & -14.63 & $<0.5 \%$ & -1.60 & $<0.5 \%$ \\
\hline Ccnb1 & -11.20 & $<0.5 \%$ & -1.64 & $<0.5 \%$ \\
\hline Ccne2 & -3.09 & $<0.5 \%$ & -1.85 & $<0.5 \%$ \\
\hline Conf & -2.63 & $<0.5 \%$ & -1.68 & $<0.5 \%$ \\
\hline Cone1 & -2.31 & $<0.5 \%$ & -2.58 & $<0.5 \%$ \\
\hline Cong1 & 1.41 & $<0.5 \%$ & -1.22 & $3 \%$ \\
\hline Ccng2 & 2.34 & $<0.5 \%$ & 1.70 & $<0.5 \%$ \\
\hline \multicolumn{5}{|c|}{ Cyclin-dependent kinase inhibitor (CDKN) } \\
\hline \multicolumn{5}{|l|}{ (arrest) } \\
\hline$C d k n 2 a$ & 1.62 & $<0.5 \%$ & 1.11 & $24 \%$ \\
\hline Cdkn1a & 1.73 & $<0.5 \%$ & 1.28 & $<0.5 \%$ \\
\hline \multicolumn{5}{|c|}{ Transforming growth factor beta (TGF $\beta$ ) (arrest) } \\
\hline$T g f b 3$ & 2.13 & $<0.5 \%$ & 1.02 & $93 \%$ \\
\hline Tgfbr2 & 1.61 & $<0.5 \%$ & 1.15 & $1 \%$ \\
\hline \multicolumn{5}{|c|}{ Checkpoint kinase (CHEK) (arrest) } \\
\hline Chek1 & -2.80 & $<0.5 \%$ & -1.37 & $<0.5 \%$ \\
\hline Chek2 & -2.47 & $<0.5 \%$ & -1.23 & $6 \%$ \\
\hline \multicolumn{5}{|c|}{ Tumor protein p53 (P53) (arrest) } \\
\hline Tp53 & -1.77 & $<0.5 \%$ & -1.79 & $<0.5 \%$ \\
\hline
\end{tabular}

\subsubsection{Cell growth regulation}

Besides the cell-cycle arrest, a continued biomass production is the other essential aspect for cell size increase. In this context, we found that in agreement with literature, the mTOR pathway is playing a central role in manipulating the cell biomass growth of the ActiCHO process. mTOR integrates the extracellular and intracellular signals through a number of upstream pathways, and regulates cell growth through its downstream effectors ${ }^{149}$. The regulation of these pathways in the ActiCHO process are summarized in Figure 4 Boxes C, D, E, and F. In addition, the significantly regulated genes involved in these pathways are shown in Table II.

First of all, the downstream effectors of mTOR are studied. The activity of these effectors are regulated by mTOR in transcriptional and/or post-transcriptional approaches. Activation of mTOR results in phosphorylation of the eukaryotic initiation factor $4 \mathrm{E}$-binding proteins (4EBPs). The phosphorylation prevents binding of these proteins to the eukaryotic initiation factor eIF $4 \mathrm{E}^{110}$. This results in increased activity of eIF4E, which promotes protein translation ${ }^{150}$. In the ActiCHO process, the eukaryotic initiation factor $4 \mathrm{E}$-binding protein $(4 \mathrm{EBP})$ genes are significantly downregulated (Eif4ebp1, FC=-2.53, FDR.BH<0.5\% and Eif4ebp2, FC=-1.40, FDR.BH<0.5\%). The down-regulation of $4 \mathrm{EBP}$ genes may have resulted in less binding to eIF4E and 
thus, released the activity of protein synthesis (see Figure 4, Box E). As a result, the protein synthesis rate in the ActiCHO process remained high during the size increase phase. In contrast, the 4EBP genes are not regulated (Eif4ebp1, FC=-1.06, FDR.BH=67\% and Eif4ebp2, FC=-1.09 FDR.BH=40\%) in the FortiCHO process, which agrees with the stopped cell growth in this process.

High mTOR activity also positively regulates the synthesis of lipids ${ }^{151}$ which is a necessary component for cell growth (see Figure 4, Box F). An increase in mTOR complex 1 (mTORC1) activity was reported to increase the sterol responsive element binding protein (SREBP) activity which led to higher lipid synthesis rate ${ }^{152}$. The higher up-regulation of the SREBP gene (Srebf1, FC=1.47, FDR.BH=3\%) in the ActiCHO process correlates with the slightly higher fatty acid synthesis rate during the cell size increase phase compared to the proliferation phase and the formation of lipid droplets, as shown in our previous study ${ }^{116}$. In contrast, the SREBP gene is not regulated ( $\mathrm{FC}=-1.02$, FDR. $\mathrm{BH}=94 \%$ ) in the FortiCHO process. In addition to by $\mathrm{mTOR}$, lipid synthesis was also shown to be induced by Akt which is a positive upstream regulator of mTOR. Akt induces lipid synthesis by up-regulating the ATP citrate lyase (encoded by Acly gene) activity. ATP citrate lyase is a key enzyme that catalyzes the reaction from citrate to cytosolic oxaloacetate and acetyl-CoA ${ }^{134}$. The latter one is a precursor for lipogenesis. In the ActiCHO process, the transcriptional activity of the Acly gene remained unchanged (FC=-1.03, FDR.BH=72\%, data to be published in Supplementary 1) whereas in the FortiCHO process, it decreased significantly $(\mathrm{FC}=-1.87$, FDR. $\mathrm{BH}<0.5 \%)$. In agreement with the transcriptome data, the fatty acids synthesis rate in the ActiCHO process was maintained more or less constant during the cell size increase phase as compared to the exponential growth phase. In contrast, the pathways related to lipid synthesis experienced significant down-regulations in the FortiCHO process, which agrees with the fact that the cells went from the exponential growth phase on day 3 to the stationary phase on day 7 since the requirement for lipids went down.

Next, cascade pathways upstream of mTOR that channel extracellular signals to mTOR were studied (Figure 4 Box C, D and F). The differentially expressed genes in these pathways are shown in Table III. The presence of extracellular growth factors and nutrients are essential to the growth and biomass synthesis of the cells. A lack of either growth factors or nutrients can lead to growth attenuation, autophagy, and eventually cell death ${ }^{153}$. The concentration of extracellular growth factors and nutrients is sensed by the cells through a number of signaling pathways. The signal of growth factors, such as insulin-like growth factors (IGF), is channeled through the PI3K-Akt pathway ${ }^{133}$. The signal transduction starts with the binding of IGF to the IGF binding protein (IGFBP, encoded by Igfbps genes) ${ }^{154}$. Next, the binding of IGF with IGFBP phosphorylates phosphatidylinositol $(4,5)$ biphosphate (PIP2) to produce PIP3 (Figure 4 Box C) ${ }^{155}$. The later one phosphorylates and activates the Akt pathway which subsequently promotes the mTOR activity, as mentioned previously. In the 
ActiCHO process, Igfbps showed stronger up-regulations (Igfbps7, FC=1.90, FDR.BH $<0.5 \%$, and Igfbps4, FC=1.51, FDR.BH<0.5\%) compared to in the FortiCHO process (Igfbps7, FC=1.15, FDR.BH=57\%, and Igfbps4, FC=-1.34, FDR.BH<0.5\%) (Table III). In agreement with this, it also can be seen in Table III that the PI3K (Pik3ip1 and $P i k 3 c 2 a)$ genes are significantly up-regulated in the ActiCHO process.

Besides the PI3K pathway, increasing evidence suggests that cytokines, such as tumor necrosis factor- $\alpha(\mathrm{TNF} \alpha)$ through the inhibitor of nuclear factor- $\kappa \mathrm{B}$ kinase- $\beta$ $(\mathrm{IKK} \beta)$ mediated pathway, can also induce the Akt activity ${ }^{135}$. It can be seen in Table III that various genes for the TNF $\alpha$ induced proteins (Tnfaip) are up-regulated much stronger in the ActiCHO process compared to in the FortiCHO process. Both the PI3K and the TNF $\alpha$-IKK $\beta$ pathways have a similar expression pattern in the ActiCHO process, and both have a positive contribution to the Akt activity. This together demonstrates that the extracellular growth factors and cytokines are likely the important factors for the activation of the Akt and subsequently the mTOR activity, meaning that they are also likely the cause of the cell size increase after the cell cycle inhibition.

In addition to the growth factors and cytokines, sufficient extracellular amino acids concentrations are needed to maintain the mammalian cell growth. The signaling pathways related to sensing the amino acid levels are illustrated in Figure 4 Box D. Sufficient amino acid levels stimulate the vacuolar $\mathrm{H}^{+}$-ATPase (v-ATPase) on the lysosomal membrane ${ }^{156}$, leading to hydrolysis of ATP by $\mathrm{v}$-ATPase. This signal stimulates the Regulator complex (encoded by the late endosomal/lysosomal adaptor, Lamtor genes) activity. The Regulator complex acts as a guanine exchange factor converting RagA/B.GDP to RagA/B.GTP ${ }^{156}$. The latter one can bind to and activate the mTORC1. As can be seen in Table III, several V-ATPase and Lamtor genes show higher up-regulation in the ActiCHO process compared to in the FortiCHO process, implying that the signals from the amino acids sensing pathways also play a role in promoting the mTOR activity for the continued biomass growth in the ActiCHO process.

Glucose is a major nutrient for $\mathrm{CHO}$ cell lines. The characteristic consumption rate of glucose and the concomitant production rate of lactate are typically high in $\mathrm{CHO}$ cell lines. In addition, as shown in Chapter $2^{8}$, the specific glucose consumption rate during the size increase phase of the ActiCHO process is significantly higher than it is during the stationary phase of the FortiCHO process. The high glucose consumption is likely the result of a high glycolysis activity and will correspondingly result in a high intracellular ATP/AMP ratio. The high ATP/AMP ratio can suppress the AMP-activated protein kinase (AMPK) activity ${ }^{157}$ and lead to an increased mTOR activity through phosphorylation (Figure 4 Box F). In this study, we are unable to confirm the state of the ATP/AMP ratio. However, one AMPK transcript (Prkaa1) showed an up-regulation in the ActiCHO process (Table III). Assuming the AMPK pathway is also regulated transcriptionally, this implies a decrease in the ATP/AMP 
ratio. In this regard, the high specific glucose consumption rate seems to play no role in activating mTOR during the cell size increase of the ActiCHO process.

Furthermore, several growth arrest specific (Gas) genes showed high (>5 FC) downregulations in the ActiCHO process (Table III). Expression of the Gas protein family is strictly related to the growth arrest state of mammalian cells ${ }^{158,159}$ (see Figure 4 Box E) and may be involved in various cellular activities including microfilaments reorganization, cell cycle, DNA synthesis, and apoptosis ${ }^{158,160,161}$. Smith and Steitz ${ }^{162}$ showed that upon reduction of the mTOR activity, Gas5 transcript levels increased. Recently, the Gas5 transcript was shown to be an important tumor suppressor involved in various cancers ${ }^{163}$. In addition, the expression of Gas2 transcript has been shown to play a critical role in preventing cancer transformation by leading the cells to a premature senescence ${ }^{164}$. In the present work, greater down-regulations of the Gas genes in the ActiCHO process correlates with the continued cell growth in the ActiCHO process. It is however not fully clear how these Gas genes interact with intra- and extracellular signals to participate in growth control.

Summarizing this section, it was shown that the mTOR upstream and downstream pathways were regulated in a strongly synchronized pattern in the ActiCHO process, leading to a higher mTOR activity in this process. In the FortiCHO process this was not observed. These results demonstrate that the activation of mTOR plays an essential role in the continued biomass growth after the stop of cell cycle progression. Furthermore, the activation of mTOR is closely related to the extracellular growth factors, cytokines, and amino acids levels, indicating that the cell size increase is related to these extracellular conditions.

Table III. Fold change (FC) mTOR upstream and downstream genes from culture day 3 to 7 in the culture with cell size increase (ActiCHO) and with no cell size increase (FortiCHO). Positive values mean upregulation and negative values mean down-regulation. Genes with a FDR.BH<5\% and the absolute FC $>1.4$ are considered significant and shown here. (Promote) means the genes promote biomass synthesis whereas (inhibit) means the genes hamper biomass synthesis.

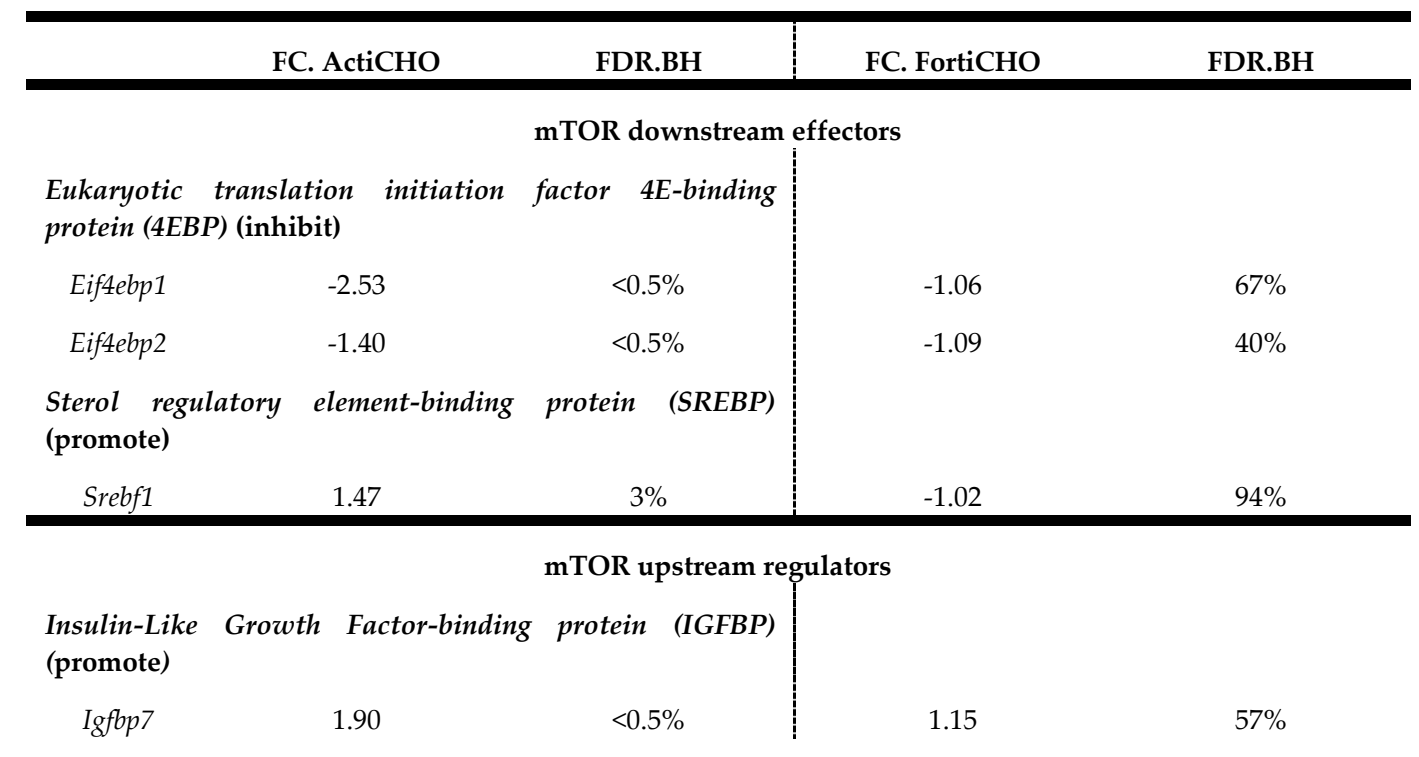




\begin{tabular}{|c|c|c|c|c|}
\hline $\operatorname{Igfbp} 4$ & 1.51 & $<0.5 \%$ & -1.34 & $<0.5 \%$ \\
\hline \multicolumn{5}{|c|}{ TNF alpha induced protein (TNFaIP) (promote) } \\
\hline Tnfaip6 & 4.21 & $<0.5 \%$ & 1.55 & $1 \%$ \\
\hline Tnfaip2 & 3.32 & $<0.5 \%$ & 1.05 & $84 \%$ \\
\hline Tnfaip8 & 1.69 & $<0.5 \%$ & -1.22 & $20 \%$ \\
\hline Tnfaip1 & 1.41 & $<0.5 \%$ & -1.06 & $64 \%$ \\
\hline Tnfaip3 & -1.43 & $<0.5 \%$ & -1.30 & $2 \%$ \\
\hline \multicolumn{5}{|c|}{ Phosphoinositide 3-kinase (PI3K) (promote) } \\
\hline Pik3ip1 & 2.01 & $<0.5 \%$ & 1.47 & $3 \%$ \\
\hline$P i k 3 c 2 a$ & 1.76 & $<0.5 \%$ & 1.01 & $94 \%$ \\
\hline \multicolumn{5}{|c|}{ Protein kinase B (PKB/AKT) (promote) } \\
\hline Akt2 & 1.40 & $<0.5 \%$ & 1.12 & $4 \%$ \\
\hline \multicolumn{5}{|c|}{ Tuberous sclerosis complex (TSC) (inhibit) } \\
\hline$T s c 2$ & 1.45 & $<0.5 \%$ & 1.11 & $18 \%$ \\
\hline \multicolumn{5}{|c|}{ Vacuolar-type $\mathrm{H}^{+}-A T P a s e$ (V-ATPase) (promote) } \\
\hline Atp6v1f & 1.64 & $<0.5 \%$ & 1.23 & $5 \%$ \\
\hline Atp6v1a & 1.62 & $<0.5 \%$ & 1.26 & $<0.5 \%$ \\
\hline Atp6v1b2 & 1.54 & $<0.5 \%$ & 1.45 & $<0.5 \%$ \\
\hline Atp6v1e1 & 1.43 & $<0.5 \%$ & 1.16 & $3 \%$ \\
\hline \multicolumn{5}{|c|}{$\begin{array}{l}\text { Late endosomal/lysosomal adaptor, MAPK and MTOR } \\
\text { activator (Lamtor) (promote) }\end{array}$} \\
\hline Lamtor 2 & 1.51 & $<0.5 \%$ & 1.08 & $58 \%$ \\
\hline Lamtor4 & 1.22 & $4 \%$ & 1.23 & $7 \%$ \\
\hline Lamtor5 & 1.2 & $3 \%$ & -1.01 & $93 \%$ \\
\hline \multicolumn{5}{|c|}{ AMP-activated protein kinase (AMPK) (inhibit) } \\
\hline Prkaa1 & 1.42 & $<0.5 \%$ & 1.08 & $32 \%$ \\
\hline \multicolumn{5}{|c|}{ Growth arrest specific (GAS) (inhibit) } \\
\hline Gas2 & -5.77 & $<0.5 \%$ & -1.95 & $<0.5 \%$ \\
\hline Gas2l3 & -5.66 & $<0.5 \%$ & -1.18 & $11 \%$ \\
\hline Gas7 & -1.78 & $<0.5 \%$ & -1.30 & $13 \%$ \\
\hline
\end{tabular}

\subsection{Extracellular stimuli for the cell size increase}

The cell size increase in this study is associated with the cell cycle arrest and the continued biomass formation. Two genes involved in osmotic regulation (Slc6a12 and $S c n 7 a$ ) showed up in the top 10 up-regulated fold changes (Table I). However, in a perfusion process using the same cell line and the ActiCHO-P medium as perfusion medium, a comparable change in cell size was observed (data not shown). In that experiment, the ActiCHO feed $\mathrm{A} / \mathrm{B}$ was not used and the culture osmolality was 
constant. It can therefore be concluded that the cell size increase is not specifically related to the ActiCHO feed A/B or osmolality.

A significant cell cycle arrest was observed in the ActiCHO process. Cell cycle arrest could be caused by secretion of toxic metabolites (e.g. ammonia and lactate) or paracrine/autocrine proteins (e.g. TGF $\beta$ ). As discussed before, ammonia and lactate are not likely the cause of the cell cycle arrest. The TGF $\beta$ pathway is significantly upregulated in the ActiCHO process, which may have caused cell cycle arrest. Another cause of the cell cycle arrest could be depletion of certain nutrients or mitogenic factors. If this was the case, these nutrients or mitogenic factors should not be present in the feeds or that the depletion causes irreversible cell cycle arrest which cannot be released by addition of the depleted nutrients anymore. This means that these factors are absent or not present in sufficient amounts in the feed. It could also be that depletion of a nutrient in the batch period causes the cells to enter a non-proliferative state from which it cannot recover by later addition of that nutrient. For example, our previous metabolic study on the ActiCHO process in Chapter $3^{116}$ identified the depletion of some non-essential amino acids including asparagine, glutamine, and tyrosine around the point where cells stopped dividing and started increasing size. Fomina-Yadlin et al. ${ }^{66}$ reported that depletion of individual amino acid can activate the amino acid response pathway (AAR), resulting in an overexpression of activating transcription factor 4 (ATF4). Consequently, cells experience down-regulation of cell cycle progression, DNA replication, nucleotide biosynthesis, and lipid metabolism. However, in the present study, the ATF4 gene (ATF4) was significantly downregulated from day 3 to 7 ( $\mathrm{FC}=-4.2$, $\mathrm{FDR} . \mathrm{BH}<0.5 \%$, data to be published in Supplementary 1), indicating that the AAR pathway was not triggered on day 7 . Since depletion occurred at day 3 and was resolved by feed addition at day 7, it could still be that a temporary overexpression between day 3 and 7 of ATF4 triggered the AAR pathway at the moment of depletion of certain amino acid(s) and caused an irreversible cell cycle arrest. In the meantime, the high concentration of the other amino acids keeps the mTOR activity high resulting in continued biomass production and cell size increase. This hypothesis, however, needs to be further confirmed by a higher time resolution for the transcriptome data and by tuning the amino acids ratio in the ActiCHO process.

\section{Concluding remarks}

We performed a transcriptome analysis to identify the molecular mechanism associated with cell size increase for a $\mathrm{CHO}$ fed-batch process. Looking at the top 10 fold-changes of up- and down-regulated genes in the ActiCHO process, genes related to redox regulation, protein transport, and osmotic regulations were highly upregulated. This result indicates that cells adapted transcriptionally to cope with redox and osmotic changes during the culture process. In addition, the top 10 down- 
regulated genes were related to cell cycle regulation and mitosis. This agrees with the observed cell size increase and cell cycle arrest in the ActiCHO process. In the next step, genes involved in cell cycle progression and biomass growth as well as in the signaling pathways of these processes were selected for further analysis. It was found that various cyclin $(c y c)$ and cyclin-dependent kinase $(c d k)$ genes were significantly down-regulated in the ActiCHO process, and a number of Cdk inhibitors (Cdkns) were significantly up-regulated, which agrees with the cell cycle arrest in both the $\mathrm{G}_{0} / \mathrm{G}_{1}$ and the $\mathrm{G}_{2} / \mathrm{M}$ phase. The gene expression results also indicate that the cell cycle arrest is not caused by DNA damage. Looking at the genes involved in regulation of biomass growth, the mTOR upstream and downstream pathways are regulated in a strongly synchronized pattern to stimulate the mTOR activity in the ActiCHO process. This agrees with a continued cell growth after cell cycle arrest. It is possible that the production of TGF $\beta$ as a negative paracrine/autocrine factor, or the depletion of certain nutrients and/or mitogenic factors in the basal medium resulted in the cell cycle arrest. Meanwhile, extracellular growth factors and high nutrients levels keep channeling the mTOR activity to maintain biomass production. This study showed that $\mathrm{CHO}$ cell size regulation is strongly related to the extracellular nutrient levels. Hence, by rational design of media and feeds, it may be possible to manipulate $\mathrm{CHO}$ cell size during industrial culture processes, which will further improve cell growth and specific productivity. 



\section{Chapter 5}

Transcriptome analysis for the scale-down of a $\mathrm{CHO}$ cell fed-batch process 
Abstract:

Transcriptome and metabolism analysis were performed to evaluate the scale-down of a $\mathrm{CHO}$ cell fed-batch process from a $10 \mathrm{~L}$ bioreactor to an ambr $15^{\circledR}$ (ambr) system. Two different agitation scale-down principles were applied, resulting in two different agitation rates in the ambr system: 1300 RPM based on the agitator tip speed, and $800 \mathrm{rpm}$ based on the volumetric power input $(\mathrm{P} / \mathrm{V})$. Culture performance including cell growth, product titer, glycosylation, and specific consumption/ production rates of metabolites was the same for both agitation rates in the ambr and was comparable to that of the $10 \mathrm{~L}$ system. The initial variation in gene expression between the inocula for the ambr and $10 \mathrm{~L}$ system was no longer present after three days of culture, indicating comparable culture conditions in both systems. Based on principal component analysis, changes in gene expression over time were similar between both scales with less than $6 \%$ variation. 2455 genes were uniquely regulated in the ambr system compared to 1604 genes in the $10 \mathrm{~L}$ system. Functional analysis of these genes did not reveal their relations with scale or cellular function. This study further strengthens that the ambr system gives representative culture performance for the $10 \mathrm{~L}$ bench-scale bioreactor.

\begin{tabular}{|c|c|}
\hline Abdulaziz A. Alsayyari a, 1 , & $\begin{array}{l}\text { a Bioprocess Engineering, Wageningen University \& Research, PO } \\
\text { Box 16, 6700 AA, Wageningen, the Netherlands }\end{array}$ \\
\hline Xiao Pan ${ }^{a}, 1$ & b Upstream Process Development, Synthon Biopharmaceuticals \\
\hline Ciska Dalm ${ }^{\mathrm{b}}$, & BV, PO Box 7071, 6503 GN, Nijmegen, the Netherlands \\
\hline Jochem $W$. van der Veen ${ }^{b}$, & $\begin{array}{l}\text { 'Biometris, Wageningen University \& Research, PO Box 16, } 6700 \\
\text { AA, Wageningen, the Netherlands }\end{array}$ \\
\hline & d Faculty of Biosciences and Aquaculture, Nord University, N- \\
\hline
\end{tabular}

René H. Wijffels a, d,

Dirk E. Martens ${ }^{a}$ *

${ }^{1}$ Both authors contributed equally to this publication 


\subsection{Introduction}

The development of a Chinese Hamster Ovary $(\mathrm{CHO})$ cell production process within the biopharmaceutical industry aims at obtaining a consistent and robust cell culture process with a high product volumetric productivity, as well as a consistent and desired product quality. To achieve this, it is important to identify the parameters that influence the culture performance and the range within which these parameters should stay to ensure a reproducible process with a consistent product quality. This requires high throughput experimentation for which small-scale bioreactors are needed. The small scale bioreactors should be representative of the large-scale so that the process developed at small-scale, including the design space for the critical process parameters, can be translated to large-scale with the same process performance. Different scale-down criteria can be used to develop a representative small-scale process. These criteria include, for example, the oxygen volumetric mass transfer rate $(\mathrm{kLa})$, the volumetric power input $(\mathrm{P} / \mathrm{V})$, and the agitator tip speed ${ }^{43,165}$. Production scale reactors can be properly scaled down to bench-scale reactors (1-10 L) which are traditionally used as the main platform for process development ${ }^{37,41}$. However, the number of experiments that can be done in a certain time frame at bench-scale is still too small for studying all the parameters that can be relevant to a culture process $(\mathrm{pH}, \mathrm{DO}$, temperature, nutrient concentrations, pre-culture age, seeding density, etc.). In order to increase the experimental throughput, several miniature bioreactor systems at $\mathrm{mL}$ scales have been developed during the past decade ${ }^{166,167}$. Like in large-scale reactors, $\mathrm{DO}, \mathrm{pH}$, agitation, and gas sparging can be monitored and controlled in these small-scale systems as well. The higher throughput allows for a shorter development time and the use of design of experiment (DoE) with a higher resolution, resulting in a more accurate definition of the design space.

The ambr ${ }^{\circledR} 15$ (from hereon called the ambr) system has advantages compared to other miniature systems due to the advanced automated operation which reduces handling errors and increases the experimental throughput. A characterization of both the ambr and large-scale stirred tank bioreactors (STRs) was conducted by Nienow et al. ${ }^{168}$. It was shown that the physical environment between the ambr and conventional STRs differs in some aspects including the flow regime, gas superficial velocity, and volumetric power input. Several studies have been performed to compare the culture performance of the ambr system to STRs ranging from $2 \mathrm{~L}$ up to $1000 \mathrm{~L}$ for $\mathrm{CHO}$ cell fed-batch processes ${ }^{169-172}$. Overall, similar process performances were reported across all scales. In a recent study done by Rouiller et al. ${ }^{173}$, it was shown that the ranking of $12 \mathrm{CHO}$ cell clones based on product titer reached in batch cultures was more similar between 3.5 L bioreactors and ambr (Pearson correlation coefficient $=0.97$ ) than between $3.5 \mathrm{~L}$ bioreactors and deep well plates and shake tubes. In the study of Janakiraman et al. ${ }^{174}$, for several studied process parameters, the ranges of these parameters which resulted in acceptable product quality attributes 
were similar between ambr and $5 \mathrm{~L}$ bioreactors. These studies show that the result of screening experiments as well as determined process parameters ranges are transferable from ambr to bench-scale systems. In contrast, Siva et al. ${ }^{175}$ showed that for a fed-batch process using a CHO DHFR- cell line the ambr gave different results compared to 5-L and 250-L STRs. Lower cell density and final titer together with an earlier decrease of cell viability were observed in the ambr system. They hypothesized that a foaming problem along with a higher ammonium concentration, a higher osmolality, and a lower $\mathrm{pH}$ in the ambr cultures were the causes of these differences. Summarizing, representative culture performances have been reported in ambr as compared to the bench-scale systems in all studies except for one and the ambr system has gained acceptance for use in process development in biopharmaceutical industries. A better understanding of the effects of the different physical environments present in the small-scale ambr system as compared to benchscale systems on cell physiology could be helpful for further validating the ambr system, specifically for developing better small-scale reactors in general.

A better understanding of cell physiology and gene regulation in relation to process conditions can be obtained using transcriptomics ${ }^{176}$. Transcriptome analysis can be used in different ways for process understanding. (i) The expression levels of genes can be used as a fingerprint to compare two process conditions, e.g. small- and largescale. Jayapal and Goudar ${ }^{30}$ used transcriptome analysis to confirm the scalability of a BHK cell perfusion culture process. (ii) The genes that are differentially expressed can be studied on a functional level. This approach can give mechanistic information on how differences in process conditions (e.g. differences between scales) influence cell physiology and process performance. For example, Sieck et al. ${ }^{32,177}$ found that transcripts related to DNA damage and repair mechanisms were up-regulated when elevated shear forces from agitation and sparging were applied to a $\mathrm{CHO}$ cell culture.

The aim of this study is to evaluate whether the physiology and performance of the cells in the ambr systems is comparable to that in a $10 \mathrm{~L}$ system for a fed-batch process. The $\mathrm{CHO}$ cells are compared on the metabolic and gene expression level. Detailed analysis of the $10 \mathrm{~L}$ process, including cell metabolism and transcriptome has been presented in Chapter $\mathbf{3}$ and $\mathbf{4}$ and was the starting point for the comparison with the ambr system in this work. $\mathrm{CHO}$ gene microarrays were used to measure the transcriptome. In addition, in order to investigate the impact of different scale-down criteria for agitation on cell physiology and culture performance, two different impeller agitation rates (800 and $1300 \mathrm{RPM})$ were included in the ambr experiment. The $800 \mathrm{RPM}$ in ambr is equivalent to the impeller volumetric power input $(\mathrm{P} / \mathrm{V})$ value that was employed at the $10 \mathrm{~L}$ scale, whereas the $1300 \mathrm{RPM}$ is equivalent to the maximum impeller tip-speed at the $10 \mathrm{~L}$ scale. 


\subsection{Materials and methods}

\subsubsection{Cell line and pre-culture}

A suspension $\mathrm{CHO}^{\mathrm{BC}}{ }^{\circledR}$ cell clone (BC-P, provided by Bioceros Holding BV) producing a recombinant immunoglobulin G1 (IgG1) was used in this study. Cells were thawed from a working cell bank and maintained in ActiCHO-P medium (GE Healthcare) supplemented with $4 \mathrm{mM}$ glutamine (Gibco, Life Technologies) and $0.5 \%$ Anticlumping agent (Gibco ${ }^{\circledR}$, Life Technologies). $200 \mu \mathrm{g} / \mathrm{mL}$ Zeocin $^{\mathrm{TM}}$ and $5 \mu \mathrm{g} / \mathrm{mL}$ Blasticidin (both from Life Technologies) were added as two selection reagents during the pre-cultures. Sub-culture was conducted in repeated batch cultures in shake flasks grown under $37^{\circ} \mathrm{C}$ and $8 \% \mathrm{CO}_{2}$ conditions. The inoculum for the $3 \times 10 \mathrm{~L}$ bioreactors was prepared in a single culture bag on a rocking bioreactor system. For each $10 \mathrm{~L}$ bioreactor, a $500 \mathrm{~mL}$ inoculum at a viable cell concentration of $3 \times 10^{6}$ cells/mL was directly inoculated in $4500 \mathrm{~mL}$ of pre-heated fresh medium, resulting in a starting density of $3 \times 10^{5}$ viable cells $/ \mathrm{mL}$ at a starting volume of $5 \mathrm{~L}$. The inoculum for the ambr bioreactors was expanded in shake flasks. To prepare the inoculum for the ambr cultures, the cells from the flasks were pooled together and spun down at $300 \times$ g. Next, the used medium was discarded and cells were re-suspended in fresh medium at a viable cell concentration of $3 \times 10^{6}$ cells $/ \mathrm{mL}$. $1.4 \mathrm{~mL}$ inoculum was inoculated in $12.6 \mathrm{~mL}$ of pre-heated fresh medium in each ambr bioreactor, resulting in a starting density of $3 \times 10^{5}$ viable cells/mL at a starting volume of $14 \mathrm{~mL}$.

\subsection{2 $10 \mathrm{~L}$ culture experiment}

Triplicate fed-batch cultures were conducted in $10 \mathrm{~L}$ Sartorius bioreactors (sartorius stedim) controlled by BIOSTAT ${ }^{\circledR}$ B-DCU II. Culture temperature was controlled at $37^{\circ} \mathrm{C}$, dissolved oxygen (DO) was controlled at $40 \%$ by pure $\mathrm{O}_{2}$ flow through a micro sparger, $\mathrm{pH}$ was controlled at 7.2 , by using base, and $\mathrm{CO}_{2}$ through a macro sparger. An air flow through the macro sparger was used to strip out the accumulating dissolved $\mathrm{CO}_{2}\left(\mathrm{pCO}_{2}\right)$. Mixing was done by an impeller at $200 \mathrm{RPM}$. From day 3 on, feeds were added to each bioreactor daily. First, the glucose concentration was measured. If the concentration was lower than $18 \mathrm{mM}$, a $45 \%$ (w/w) glucose solution was added as one bolus to reach a glucose concentration of $28 \mathrm{mM}$ in the reactor. Next, 4.5\% (v/v) ActiCHO feed A, also containing about $500 \mathrm{mM}$ glucose amongst other nutrients, and $0.45 \%$ (v/v) ActiCHO feed B (both from GE Healthcare, USA) per culture volume per day were fed to each reactor. Simethicone antifoam solution was added to each bioreactor prior to inoculation and during the culture when needed.

For each $10 \mathrm{~L}$ bioreactor, a $15 \mathrm{~mL}$ sample was taken daily before and after feed addition. Total cell density, viable cell density, and cell diameter were measured using a CedexHiRes ${ }^{\circledR}$ analyzer (Innovatis). $3 \mathrm{~mL}$ of the sample was spun down at 
300×g for 5 min (Heraeus Multifuge X3R, Thermo Scientific). The cell pellet was collected and the total RNA was extracted using $3 \mathrm{~mL}$ TRIzol reagent (Invitrogen ${ }^{\mathrm{TM}}$ ) and stored at $-80^{\circ} \mathrm{C}$ for later transcriptome analysis. The remaining sample was spun down at $3345 \times \mathrm{g}$ for $15 \mathrm{~min}$. $1 \mathrm{~mL}$ supernatant from the sample was measured for offline $\mathrm{pH}, \mathrm{pCO} 2$, glucose, lactate, ammonium concentrations and osmolality by a nova BioProfile FLEX analyzer (nova biomedical). The remaining supernatant was stored at $-20{ }^{\circ} \mathrm{C}$ for later analysis. On culture day 4, 7 and 10 biomass samples were taken. The preparation of the biomass samples was performed in the same way as in Chapter $3^{116}$.

\subsection{3 ambr culture experiment}

The ambr system used in this study consisted of 4 culture stations (CS), each CS contained 12 bioreactors. In total 48 bioreactors were used in this study. The reactor volume was kept between 13 and $15 \mathrm{~mL}$ throughout the culture. For all $4 \mathrm{CSs}$, the temperature was controlled at $37^{\circ} \mathrm{C}$, dissolved oxygen (DO) was controlled at $40 \%$ by oxygen enrichment in gas supply through an open pipe sparger, and $\mathrm{pH}$ was controlled by $0.5 \mathrm{M} \mathrm{NaOH}$ and $\mathrm{CO}_{2}$ enrichment in gas supply. $\mathrm{pH}$ was measured offline on a different analyzer as the $10 \mathrm{~L}$ cultures, because of the smaller sample volume. Measurement differences in $\mathrm{pH}$ due to the different analyzer used were accounted for creating matching bands of control. The daily feeding strategy was similar as in the $10 \mathrm{~L}$ culture experiment with a difference that the feed can only be added at one time as a bolus. $4 \times$ diluted simethicone antifoam solution was added to each ambr bioreactor when needed. Two different agitation rates were tested in the ambr culture experiment being: CS-1 and CS-2 at 800 RPM which is equivalent to the volumetric power input $(\mathrm{P} / \mathrm{V})$ value of the $10 \mathrm{~L}$ culture experiment, and CS-3 and CS4 at 1300 RPM which is equivalent to the agitator's tip-speed of $10 \mathrm{~L}$ culture experiment (Table I). The $\mathrm{P} / \mathrm{V}$ value for the $10 \mathrm{~L}$ reactor is obtained from:

$$
\frac{P}{V}=\frac{P_{0} \rho N^{3} D^{5}}{V} \quad \text { Equation } 1
$$

where $\mathrm{P}$ is the power input from impeller $(\mathrm{W}), \mathrm{V}$ is the culture volume $\left(\mathrm{m}^{3}\right), \mathrm{P}_{0}$ is the power number of the $10 \mathrm{~L}$ bioreactor impeller and was determined as 2.1 based on Van't Riet, K. and Tramper, J.22, $\mathrm{Q}$ is the liquid density $\left(\mathrm{kg} / \mathrm{m}^{3}\right), \mathrm{N}$ is the impeller speed $\left(\mathrm{s}^{-1}\right), \mathrm{D}$ is the impeller diameter $(\mathrm{m})$. The Reynolds number $(\mathrm{Re})$ and the Kolmogorov length $\left(\lambda_{k}, \mu \mathrm{m}\right)$ scale in a turbulent flow are calculated based on Van't Riet, K. and Tramper, J.22:

$$
\begin{gathered}
R e=\frac{\rho N D^{2}}{\eta} \quad \text { Equation } 2 \\
\lambda_{k}=\left(\frac{P}{\rho V \vartheta^{3}}\right)^{-\frac{1}{4}} \quad \text { Equation } 3
\end{gathered}
$$

where $\eta$ is the dynamic viscosity $\left(\mathrm{N} \cdot \mathrm{s} \cdot \mathrm{m}^{-2}\right)$, and $\vartheta$ is the kinematic viscosity $\left(\mathrm{m}^{2} \cdot \mathrm{s}^{-1}\right)$. 

used in this study. It is shown for comparison only.

\begin{tabular}{|c|c|c|c|c|c|c|c|}
\hline & $\begin{array}{l}\text { Agitation } \\
\text { rate (RPM) }\end{array}$ & $\begin{array}{l}\text { Tip- } \\
\text { speed } \\
(\mathrm{m} / \mathrm{s})\end{array}$ & $\begin{array}{c}\mathrm{P} / \mathrm{V} \\
(\mathrm{W} / \mathrm{m} 3)\end{array}$ & $\begin{array}{l}\text { kLa } \\
(/ h)\end{array}$ & $\operatorname{Re}$ & Flow & $\begin{array}{c}\text { Kolmogorov } \\
\text { (um) }\end{array}$ \\
\hline \multirow{2}{*}{ ambr } & 800 & 0.48 & $65^{*}$ & $4-6^{*}$ & 2500 & Laminar & - \\
\hline & 1300 & 0.78 & $280^{*}$ & $5-8^{*}$ & 4000 & Transient & 27 \\
\hline \multirow{2}{*}{$10 \mathrm{~L}$} & 200 & 0.82 & 40 & \multirow{2}{*}{$2-40$} & 29000 & \multirow{2}{*}{ Turbulent } & 55 \\
\hline & 300 & 1.23 & 135 & & 43000 & & 40 \\
\hline
\end{tabular}

*: values adopted from Nienow et al. ${ }^{168}$

Maximum $800 \mu \mathrm{L}$ daily sample volume was taken before feed addition from each individual ambr bioreactor. Individual ambr bioreactors were sampled every other day to measure off-line $\mathrm{pH}, \mathrm{pCO}_{2}$, glucose concentration, and lactate concentration on a blood gas analyzer (ABL90 FLEX). Larger samples were obtained daily from paired cultures run under identical conditions which were analyzed for metabolites, cell density and culture viability, IgG concentration, spent media, biomass composition, and RNA preparation by using the same methods as for the $10 \mathrm{~L}$ cultures.

\subsubsection{Biomass, spent medium, and mAb analysis}

The analysis of biomass composition including cell dry weight, soluble cellular protein, fatty acids, and carbohydrate was performed using the same method as described in Chapter $3^{116}$. Compositions of the spent medium including extracellular amino acids and organic acids were quantified using NMR (Spinnovation Biologics BV). mAb (IgG1) titer was quantified by using Protein-A chromatography (Agilent, 5069-3639). mAb N-glycan composition was analyzed by a Hydrophilic Interaction Chromatography (HILIC UPLC). The methods for mAb quantification and N-glycan analysis were described in Chapter $3^{116}$.

\subsubsection{Average specific metabolic rates}

The calculation of the specific production/consumption rate of a compound is the same as described in Chapter $\mathbf{3}^{116}$. In brief, the following equation is used, assuming a constant $\mathrm{q}_{\mathrm{x}}$

$$
M_{x}(t)-M_{x}(0)-V_{f} \times C_{f}=q_{x} \times \int_{0}^{t} X_{V C} d t \quad \text { Equation } 4
$$

where $\mathrm{M}_{\mathrm{x}}(\mathrm{mg} ; \mathrm{mmol})$ is the total amount of compound $\mathrm{x}$ in a culture at time 0 and $\mathrm{t}$, $\mathrm{X}_{\mathrm{vc}}$ (cells) is the number of viable cells in a culture, $\mathrm{V}_{\mathrm{f}}\left(\mathrm{mm}^{3}\right)$ is the total volume of feed added, $C_{f}(\mathrm{mM})$ is the concentration of compound $x$ in the feed, and $q_{x}\left(\mathrm{mg} \cdot\right.$ cell $^{-}$ 


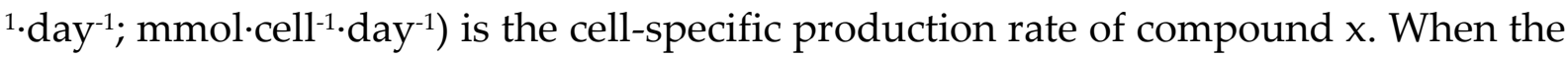
rates are calculated based on cell volume, $X_{\mathrm{vc}}\left(\mathrm{mm}^{3}\right)$ presents the volume of viable cells in a culture and $\mathrm{q}_{x}\left(\mathrm{mg} \cdot \mathrm{mm}^{-3} \cdot \mathrm{day}^{-1}\right.$ or $\left.\mathrm{mmol} \cdot \mathrm{mm}^{-3} \cdot \mathrm{day}^{-1}\right)$ is the cell volumespecific production rate of compound $\mathrm{x}$. The average specific production rates $(\mathrm{q})$ were obtained from the slope of a plot of accumulated consumed/produced compound mass against the accumulated integral of viable cell number (or the integral of viable cell volume) using linear regression.

\subsubsection{Transcriptome analysis}

For both scales, the transcriptome analysis was carried out on: cells from the inoculum just before inoculation, on day 3 which represents the exponential phase, on day 5 which represents the cell size increase phase, and on day 9 which represents the stationary phase. For the inoculum of the $10 \mathrm{~L}$ experiment, three samples were taken from the culture bag before inoculation. For the inoculum of the ambr experiment, three samples were taken from the pooled shake flasks just before centrifugation. During the culture, for the $10 \mathrm{~L}$ experiment, three samples were taken from the three individual bioreactors, whereas for the ambr experiment, also three samples were taken with each sample obtained by pooling the samples from two bioreactors of the 800 RPM condition. The detailed approach for transcriptome analysis is described in Chapter 4. Genes regulation that satisfied the criterion of FDR.BH $<0.05$ and absolute fold-change (FC) $>1.4$ were defined to be significant and were subjected to KEGG pathways over-representation analysis using fisher's exact test and Venn diagram. The raw data on transcriptome analysis is data to be published in Supplementary A of the online published version.

\subsection{Results and discussion}

\subsubsection{DO and $\mathrm{pH}$ control}

Proper control of dissolved oxygen (DO) and $\mathrm{pH}$ is critical for the culture performance. The online $\mathrm{DO}$ and $\mathrm{pH}$ profiles of a representative ambr reactor and a representative $10 \mathrm{~L}$ reactor are shown in Figure 1. For the ambr cultures, the DO and the $\mathrm{pH}$ were well controlled around the set-point. However, compared to the $10 \mathrm{~L}$ cultures, the ambr cultures showed higher spikes of DO and pH. In addition, slightly higher $\mathrm{pH}$ values from day 5 to 8 can be seen in the ambr cultures. The higher spikes of DO were caused by the opening of the vessels which was needed for feed addition, base addition, and sampling. The spikes in $\mathrm{pH}$ in both the ambr and the $10 \mathrm{~L}$ cultures were caused by the daily addition of ActiCHO feed B, which has a high $\mathrm{pH}$ value of 11. The spikes were higher in the ambr cultures, due to the fact that for the ambr the feed was added as a bolus whereas for the $10 \mathrm{~L}$ it was added over a period of 2 hours. 

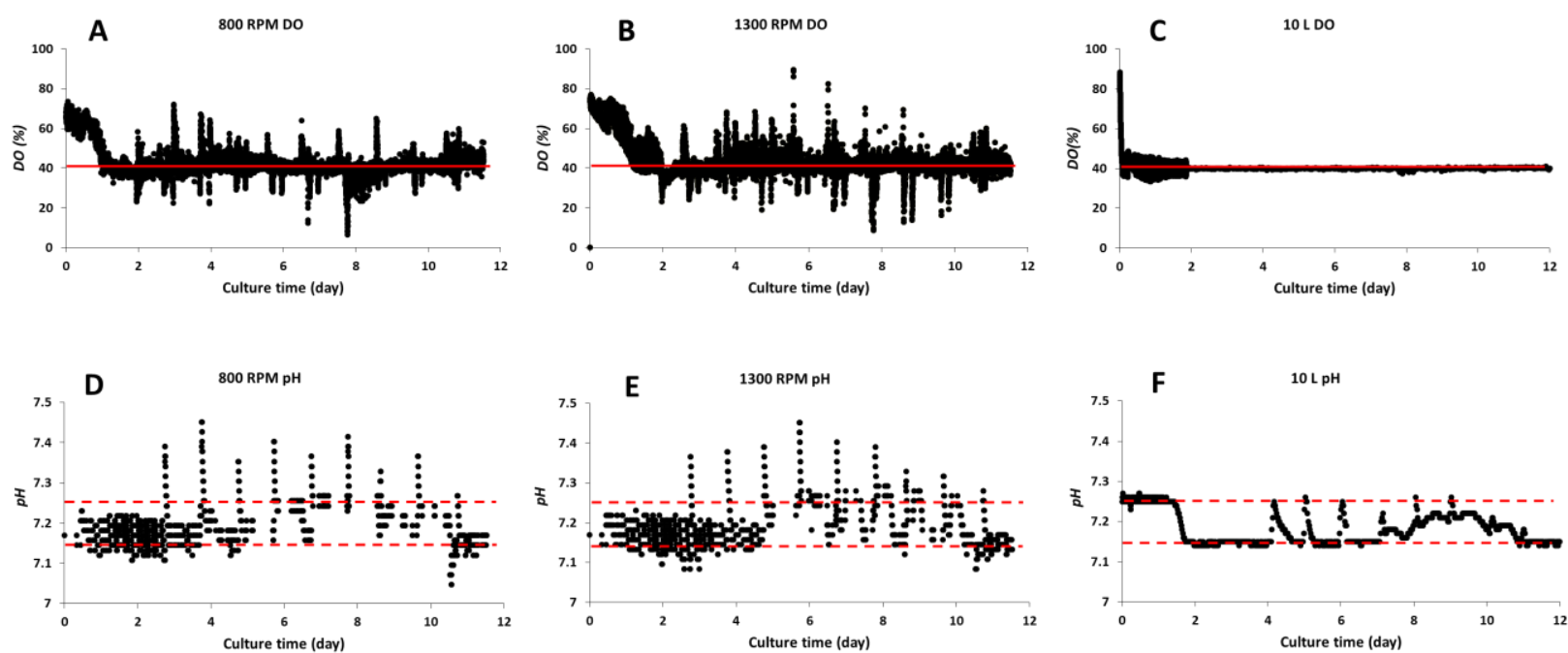

Figure 1. Examples of the online measured $\mathrm{pH}$ and $\mathrm{DO}$ profiles. Graph $\mathrm{A}, \mathrm{B}$, and $\mathrm{C}$ show DO profiles of the ambr 800 RPM, the ambr 1300 RPM, and the 10 L bioreactor, respectively. Graph D, E, and F show pH profiles of ambr 800 RPM agitation, ambr 1300 RPM agitation, and $10 \mathrm{~L}$, respectively. For DO the set-points are shown by red solid lines, and for $\mathrm{pH}$ the dead-bands are shown in red dashed lines. The differences in $\mathrm{pH}$ measurement due to the different analyzers used between the scales were corrected for as mentioned in materials and methods 5.2.2 and 5.2.3.

\subsubsection{Cell growth}

The cell growth characteristics including viable cell density (VCD), viability, and average cell diameter are shown in Figure 2 for the two agitation rates of the ambr experiment, and the $10 \mathrm{~L}$ experiment. As described in Chapter $3^{116}$, the fed-batch culture can be divided into a cell number increase phase (NI phase, day 0-4), a cell size increase phase (SI phase, day 4-8), and a stationary phase followed rapidly by a death phase (day 8-12). Similar cell growth and viability profiles were observed between the two agitation conditions in the ambr cultures. Compared between the two scales, the ambr cultures resulted in a slightly higher peak VCD $\left(\sim 8 \times 10^{6} \mathrm{cells} / \mathrm{mL}\right)$ than the $10 \mathrm{~L}$ cultures $\left(\sim 7 \times 10^{6}\right.$ cells $\left./ \mathrm{mL}\right)$. Due to a difference in inoculation method (see section 2.1), the ambr cultures are started with $100 \%$ fresh medium whereas the $10 \mathrm{~L}$ cultures are started with medium that contains $10 \%$ spent medium from the inoculum. This led to lower starting nutrient concentrations in the $10 \mathrm{~L}$ cultures and most likely a lower maximum VCD in the $10 \mathrm{~L}$ cultures. This difference is however not associated with the bioreactor system and can easily be solved by also adding the pre-culture directly to the ambr reactor without replacing the medium. Apart from this, the profiles for viability, cell growth, and cell diameter are comparable between the two scales. 
A

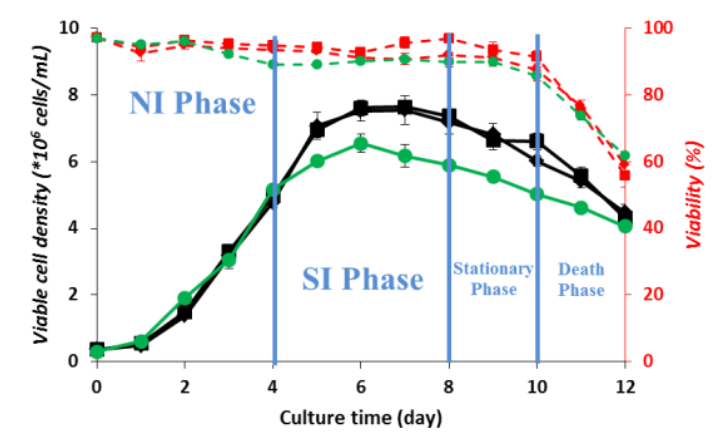

B

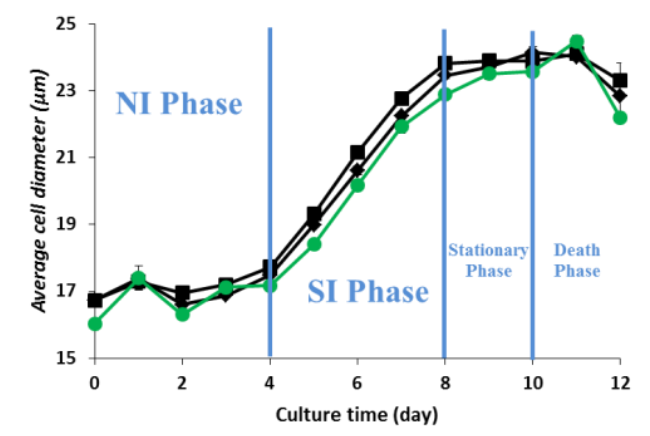

$\begin{array}{ll}\rightarrow-800 \mathrm{RPM} & \longrightarrow 1300 \mathrm{RPM} \\ --800 & -10 \mathrm{~L}\end{array}$

Figure 2. A. Viable cell density (VCD, $10^{6}$ cells $\left./ \mathrm{mL}\right)$ and viability (\%), and B. average cell diameter ( $\left.\mu \mathrm{m}\right)$ of ambr 800 RPM (closed square, black lines), ambr 1300 RPM (closed diamond, black lines), and $10 \mathrm{~L}$ (close circle, green lines) cultures. The error bars represent the standard deviation for ambr ( $n=5$ for each condition) and $10 L(n=3)$ cultures.

\subsection{3 mAb production and glycan distribution}

Product characteristics including product titer, cell-volume based specific productivity, and N-glycan distribution are presented in Figure 3 for the two agitation conditions of the ambr and $10 \mathrm{~L}$ cultures. In Chapter $\mathbf{3}^{116}$, it was shown that the specific productivity increases linearly with the cell volume. The cell-volume specific productivity is constant during the whole process and therefore for comparison of the specific productivity the cell volume-based specific productivity is used instead of the cell-based value. First of all, the two agitation conditions in the ambr gave similar product characteristics. Compared between scales, the ambr cultures yielded a slightly higher $\mathrm{mAb}$ titer $(750 \mathrm{mg} / \mathrm{L})$ than the $10 \mathrm{~L}$ cultures $(650$ $\mathrm{mg} / \mathrm{L}$ ) (Figure 3A). However, the cell volume-based specific mAb productivity (Figure 3B) was the same between the two scales, meaning that the difference in $\mathrm{mAb}$ titer was caused by the difference in viable cell density, since the volume per cell is comparable between scales (Figure 2). A difference in the N-glycan distribution is observed at culture day 12 between the ambr and $10 \mathrm{~L}$ cultures, represented by a higher fraction of G0 and G1, and a lower fraction of G0F for the ambr cultures compared to the $10 \mathrm{~L}$ cultures (Figure 3C). A survey from the historical data using the same cell line and culture media system (not published data from the Bioprocess Engineering group of Wageningen University) showed a $10-20 \%$ variation of the mAb N-glycan species, which could be caused by the difference in the pre-culture batches and in the sampling time. Considering this variation, the difference in $\mathrm{mAb}$ glycosylation between the ambr and $10 \mathrm{~L}$ experiment in this study (Figure 3C) is minor. 

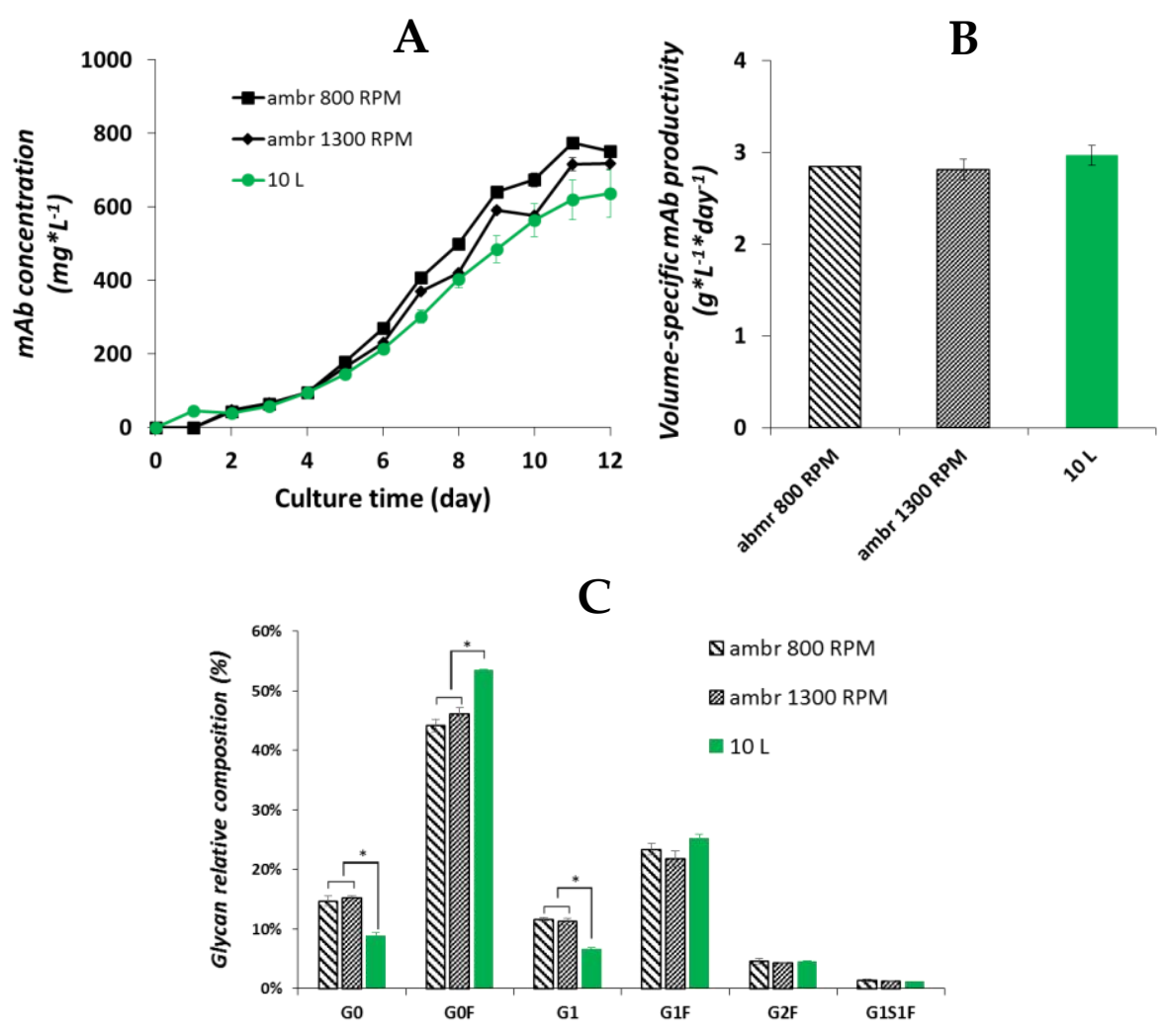

Figure 3. A. mAb concentration ( $\left.m g \times L^{-1}\right)$ of ambr 800 RPM (closed square, blue line), ambr 1300 RPM (closed diamond, blue line), and $10 \mathrm{~L}$ (close circle, green line) cultures. B. Cell volume-specific $\mathrm{mAb}$ productivity $\left(\mathrm{g} \times \mathrm{L}^{-1} \times \mathrm{day}^{-1}\right)$. C. Relative $\mathrm{mAb} \mathrm{N}$-glycan composition (\%) of ambr $800 \mathrm{RPM}$, ambr $1300 \mathrm{RPM}$, and $10 \mathrm{~L}$ cultures of the culture day $12 . \mathrm{N}$-glycans with different numbers of terminal residuals (G: galactose, $F$ : fucose, S: sialic acid) are shown. *: $P<0.05$, $T$-test. The error bars represent the standard deviation $(n=3)$ of biological triplicates.

\subsubsection{Metabolite profiles}

The primary metabolite concentrations and osmolality profiles are shown in Figure 4 for the two agitation conditions tested in the ambr, and in the $10 \mathrm{~L}$ cultures. The other measured metabolite concentrations are data to be published in Supplementary B. The two agitation conditions in the ambr experiment gave similar results, only the $\mathrm{pCO}_{2}$ profile was lower at $800 \mathrm{RPM}$ from day 5 onwards. This was probably the result of increased $\mathrm{CO}_{2}$ stripping due to the fact that the $800 \mathrm{RPM}$ condition required a higher gas flow rate compared to the 1300 RPM condition to maintain the DO at the set-point. The higher gas flow rate was needed because of the lower oxygen transfer from the headspace due to the lower agitation rate. 

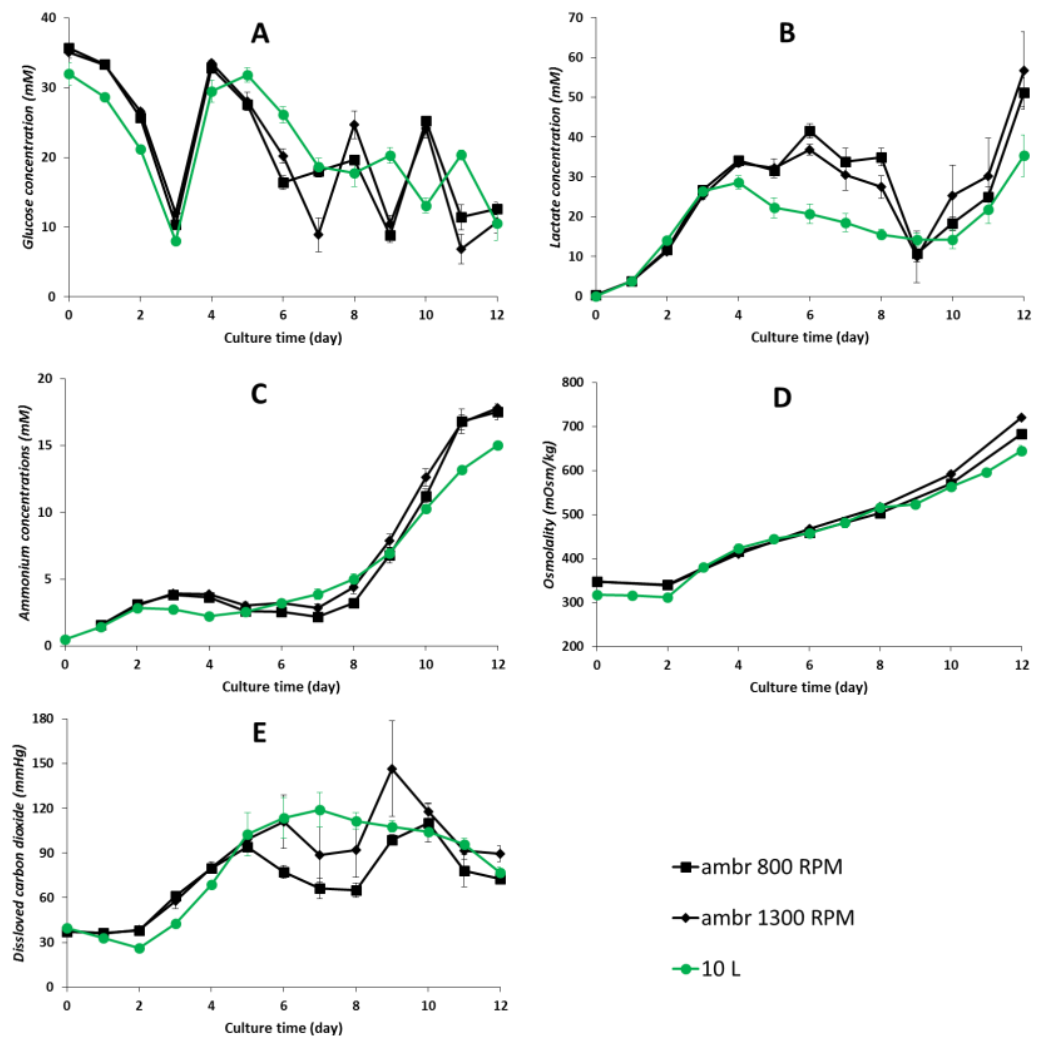

Figure 4. Profiles of the extracellular glucose concentration (mM) (A), lactate concentration (mM) (B), ammonium concentration $(\mathrm{mM})(\mathrm{C})$, osmolality $(\mathrm{mOsm} / \mathrm{kg})(\mathrm{D})$, and dissolved CO2 $(\mathrm{mmHg})(\mathrm{E})$ of ambr 800 RPM (closed square), ambr 1300 RPM (closed diamond), and 10 L (closed circle) cultures. All measurements that were done using different methods were corrected between the two scales. The error bars show standard deviation for ambr ( $n=5$ for each agitation condition) and $10 \mathrm{~L}(\mathrm{n}=3)$ cultures.

The glucose concentration profiles showed a similar pattern between the two scales. Note that the slightly lower glucose concentration and osmolality on the first days in the $10 \mathrm{~L}$ bioreactor were caused by the different treatments in inoculum media as explained before. The lactate concentration for both scales showed a similar increase until day 3. From day 4 to day 8 , however, the lactate concentration was up to $20 \mathrm{mM}$ higher in the ambr as compared to in the $10 \mathrm{~L}$ cultures. This was a consequence of both the higher cell density (Figure 2A) and the higher specific lactate productivity (Figure 5a) in the ambr as compared to in the $10 \mathrm{~L}$ culture. After day 9, all cultures showed a sharp increase in the lactate concentration again, which coincided with the decrease in viability (Figure 2). The profiles of the ammonium concentration and osmolality were similar between the two scales. The culture osmolality increased from day 3 on for all conditions due to the feed addition. $\mathrm{pCO}_{2}$ levels were comparable until day 5. From day 5 to day 9, a difference in the $\mathrm{pCO}_{2}$ level was observed between the two scales. The $\mathrm{pCO}_{2}$ in the ambr $1300 \mathrm{RPM}$ cultures is more comparable to the $10 \mathrm{~L}$ cultures and they are both higher than in the ambr $800 \mathrm{RPM}$ cultures. 


\subsubsection{Cell metabolism}

Small differences can be seen in the extracellular product (Figure 3A), lactate (Figure 4B), and nutrient (Supplementary B of the online published version) concentrations between the two scales, which might be caused by the small difference in viable cell density. In order to compare the two scales at the cellular level we investigated the metabolism, by looking at specific metabolite consumption and production rates, and the biomass composition. The specific rates are the same for the two agitation rates in the ambr (data not shown). This demonstrates that the two agitation rates calculated based on two scale-down criteria did not cause a difference in cell metabolism. Therefore, in this section, only the ambr 800 RPM condition is shown and compared with the $10 \mathrm{~L}$ bioreactor.

\subsubsection{Specific consumption/production rates}

The cell specific rates of the primary metabolites and amino acids are compared between the ambr and $10 \mathrm{~L}$ cultures for the NI phase SI phase in Figure 5. In Chapter $3^{116}$, it was shown that the cell specific rates are more or less constant despite the increase in volume. Slightly higher specific lactate production and consumption rates are observed in the ambr cultures compared to the $10 \mathrm{~L}$ cultures (Figure 5A). A possible cause was the different $\mathrm{pH}$ and $\mathrm{DO}$ profiles in these two systems (Figure 1). First of all, slightly higher $\mathrm{pH}$ values from day 5 to 8 and $\mathrm{pH}$ spikes towards higher values were observed in the ambr cultures (Figure 1D\&E), which may favor the transport of lactate out of cells ${ }^{178}$. Furthermore, it was reported by Serrato et al. ${ }^{179}$ that compared to a constant DO, oscillating DO values resulted in an increased glycolytic metabolism and an increased lactate yield. Next to the specific rate of lactate production, minor differences in specific rates between the two scales can be observed for ammonium during the NI phase, and for a few amino acids in both the NI and the SI phase (Figure 4B). Overall, the specific metabolic rates of these primary metabolites are very comparable between the two scales. 


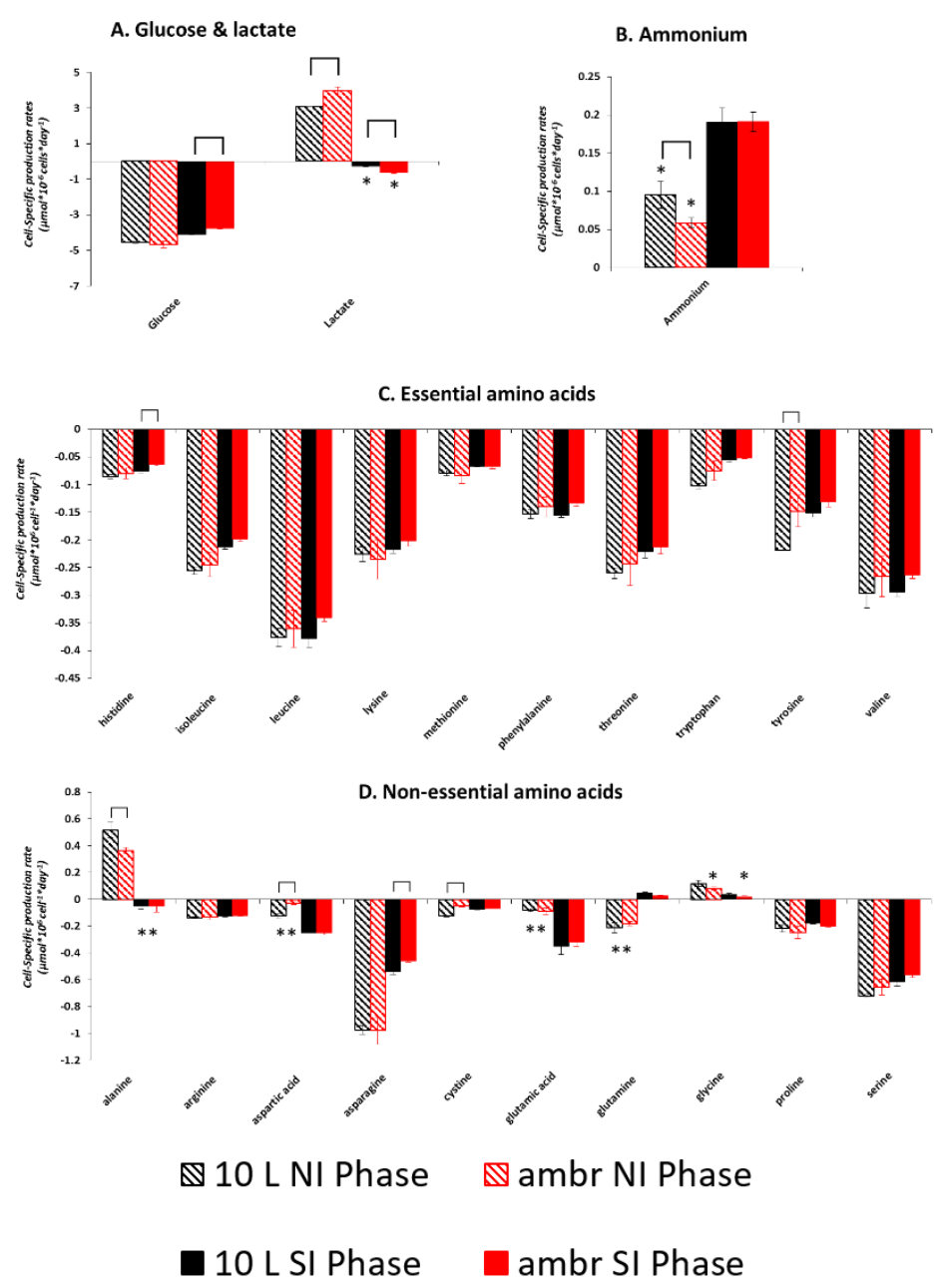

Figure 5. Average cell-specific production rates $\left(\mu \mathrm{mol} \times 10^{6}\right.$ cell $\left.^{-1} \times d a y^{-1}\right)$ of glucose and lactate $(A)$, ammonium (B), essential amino acids $(C)$, and non-essential amino acids $(D)$ of the 10 L (black) and the ambr (red) cultures during the number increase (NI, stripes), and the size increase (SI, filled) phase. Positive values indicate production, negative values indicate consumption. The error bars show the standard deviation $(n=3)$ of biological replicates. * shows the rate was not constant over the measurement points in time $\left(\boldsymbol{R}^{2}<\right.$

0.9). "「" shows a significant difference $(P<0.05$, T-test) between the values calculated from the two compared scales.

\subsubsection{Biomass composition}

The cell size increased during the fed-batch process (Figure 2B). In Chapter $\mathbf{3}$ it was shown that the biomass composition changes when the cells increase in size ${ }^{116}$. The biomass composition between two scales is compared on day 7 that represents the cell size increase phase. In addition to biomass dry weight (DW), the relative composition $(\mathrm{w} / \mathrm{w})$ of several main biomass components were measured including protein, fatty acids, and carbohydrates (Table II). The results show identical biomass composition between the $10 \mathrm{~L}$ and the ambr cultures on culture day 7. Together with the similar cell diameter profiles (Figure 2B), this indicates highly comparable biomass dynamics between $10 \mathrm{~L}$ and ambr cultures. 


\begin{tabular}{cccccc}
\hline & \multicolumn{2}{c}{$10 \mathrm{~L}$} & \multicolumn{3}{c}{ ambr } \\
\hline Dry weight (pg/cell) & 1530 & \pm 77 & 1483 & \pm 43 \\
Protein (w/w \%) & 70.0 & \pm 1.2 & 71.3 & \pm 1.3 \\
Fatty acids (w/w \%) & 4.5 & \pm 0.1 & 4.4 & \pm 0.2 \\
Carbohydrates (w/w \%) & 3.8 & \pm 0.2 & 3.9 & \pm 0.1 \\
\hline
\end{tabular}

士: Standard deviation obtained from the results of three independent bioreactors

\subsubsection{Transcriptome analysis}

Gene expression was measured using $\mathrm{CHO}$ Gene microarrays of the cells in the inocula just before inoculation and on day 3, 5, and 7 in both systems in triplicate. For the transcriptome study, only the 800 RPM condition of the ambr experiment is used to compare to the $10 \mathrm{~L}$ experiment. First, principal component analysis (PCA) is used to study the global variation in gene expression between the two scales. Next, functional analysis is performed for the genes that are uniquely regulated in one of the systems in order to study their relationship with the differences between the two bioreactor systems and with cell behavior.

\subsubsection{Global variation analysis in gene expression}

The principal component analysis (PCA) result is presented in Figure 6A\&B, which shows the overall variation in gene expression for all analyzed samples including the inocula. The inocula are included at first because the difference in the inocula may cause differences in cell behavior between both systems. The principal components (PC) PC1, PC2, and PC3 represent 33\%, 10\%, and 6\% of the total variation, respectively. The other individual PCs have only a minor contribution (less than $5 \%$ per individual component) to the total variation and are not considered. The variance explained by PC1 correlates with the culture development over time. For the variance explained by $\mathrm{PC} 2$, no clear unique correlation to the culture performance can be found. PC3, which represents $6 \%$ of the total variance, mainly explains the variance between the inocula. After three days of culture, both systems group together on all three principle components and the variance in gene expression observed in the inocula has disappeared. 


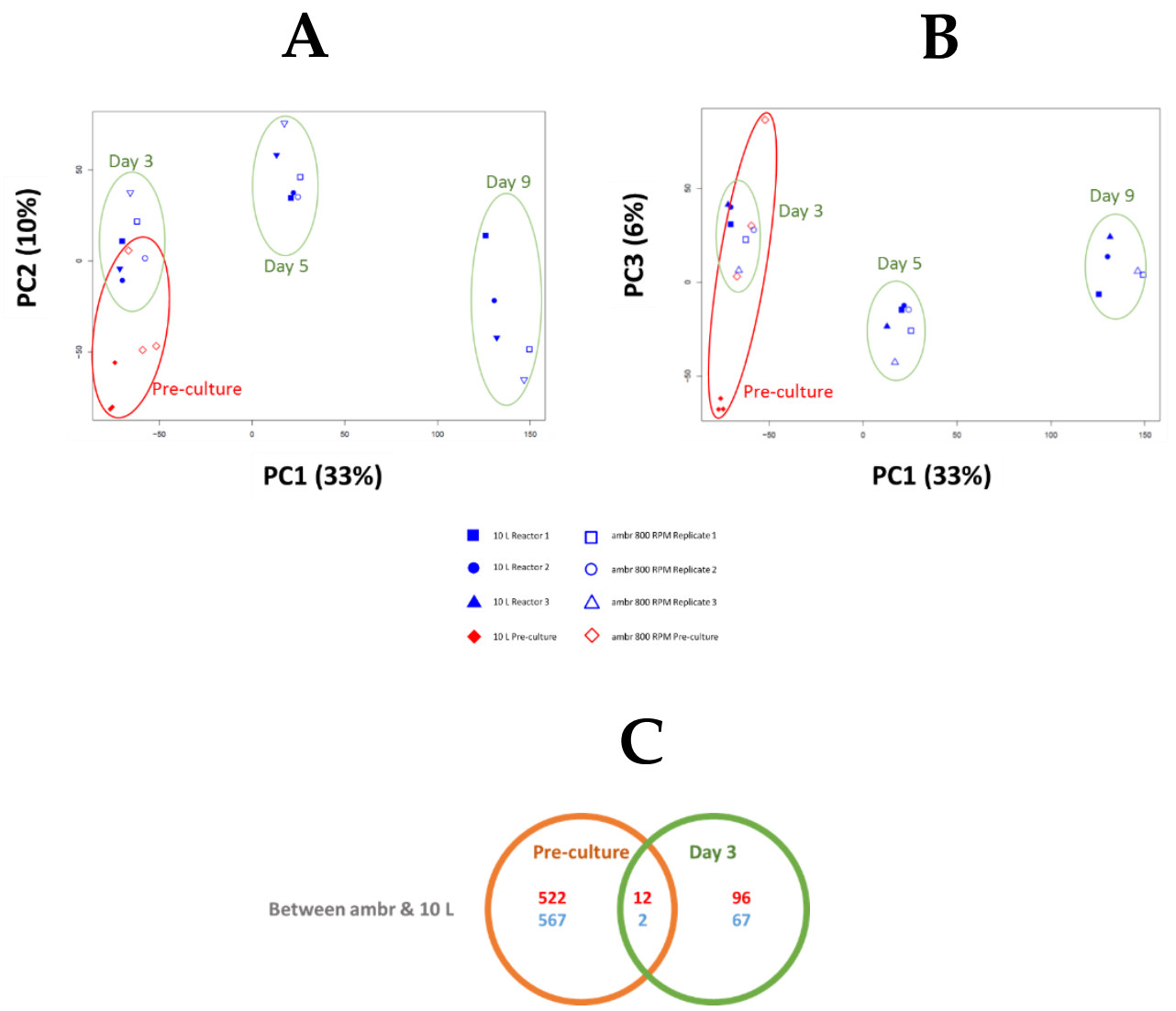

Figure 6. $(A \& B)$ Score plots generated from the transcriptome results of the $10 \mathrm{~L}$ (filled markers), ambr experiments with 800 RPM agitation rate (open markers), and pre-cultures at the moment of inoculation (filled diamond: pre-culture 10 L reactor, open diamonds: pre-culture ambr reactor). Samples for pre-cultures are grouped by red circles. Samples on the same culture day are grouped by green circles. On day 9, one replicate of the ambr culture was excluded due to a technical error in the microarray. (C) Venn diagram of the number of genes that are differentially regulated between the ambr and $10 \mathrm{~L}$ cultures for the pre-culture samples and the samples on day 3.

The genes that are significantly differentially expressed (FDR.BH $<0.05$ and absolute FC $>1.4$, see section 2.6) between the two pre-cultures are compared to those that are significantly differentially expressed between both systems on day 3 (Figure 6C). Between the two inocula, 1089 genes (total number of genes in the pre-culture circle) were differentially expressed, while between the two systems on day 3, only 167 genes (total number of genes in the day 3 circle) were differentially expressed. Moreover, of the genes that were differentially expressed between the inocula, only 14 were also differentially expressed between both systems on day 3 . Thus the differences in gene expression on day 3 involve different genes than those that caused differences between the inocula. In conclusion, the difference in gene expression between the inocula probably did not cause differences in gene expression between the two scales later on and thus did not influence the scale comparison.

Next, the differences in gene expression between the ambr and $10 \mathrm{~L}$ system are studied by comparing the gene expression data from day 3, 5, and 9, excluding the 
inocula samples. The results of PCA are shown in Figure 7. PC1, PC2, and PC3 now represent $37 \%, 10 \%$, and $6 \%$ of the total variation, respectively. The other individual PCs each have only a minor contribution (less than $5 \%$ per individual component) to the total gene expression variance and are not considered. Samples taken on the same culture day of the two scales are closely grouped on PC1 and PC2 (Figure 7A). PC1 again correlates with the culture development over time. PC2 shows a transient pattern that correlates with the cell size increase, since the cell size increased on day 5 but not on day 3 and 9. Based on PC1 and PC2 there is a slight separation of the ambr and $10 \mathrm{~L}$ cultures on day 3 and day 9. When looking at the development in time as represented by PC1 moving to the right and PC2 moving to a minimum on day 5, it seems that the ambr system is slightly ahead of the $10 \mathrm{~L}$ cultures in its development. In agreement with this, slightly larger cell diameters during the SI and the stationary phase are observed in the ambr cultures (Figure 2B). PC3 relates to the difference between the two scales. As can be seen the difference becomes more prominent for the later stage of the cultures (day 5 and 9). PC3 describes $6 \%$ of the total variance, which is much less than the variance that is due to the changes in gene expression over time $(47 \%$, PC1+PC2). To analyze whether this global difference in gene expression between the scales is relevant for the evaluation of the scale-down, the function of the genes that are differentially expressed between both systems were subsequently studied in more detail.
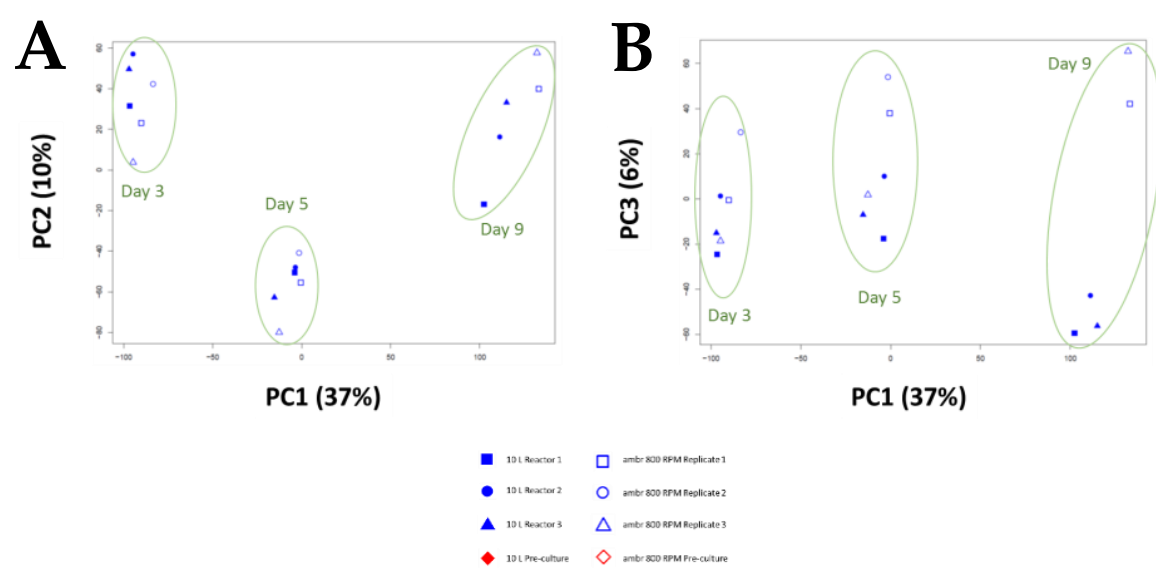

Figure 7. Score plots for the transcriptome results of the $10 \mathrm{~L}$ (filled markers) and ambr experiments with 800 RPM agitation rate (open markers) on day 3, 5, and 9. On day 9, one replicate of the ambr culture was excluded due to a technical error in the microarray.

\subsubsection{Gene functional analysis}

For the functional analysis, first, for each system the genes that are significantly differentially (as shown in section 2.6) expressed from day 3 to 5, day 5 to 9 , and day 
3 to 9 are selected. Genes are considered differentially expressed between two days if the FDR.BH is smaller than 0.05 and the absolute fold-change (FC) is larger than 1.4 (section 2.6). Next, the differentially expressed genes over time are compared between the ambr and 10L system and the result is shown in Figure 8. Note that the day 3-9 comparison picks up genes for which the expression level goes up or down slowly in time such that they were still insignificant in the 3-5 and 5-9 time frames. However, the day 3-9 time frame misses genes that are first up-regulated and then down-regulated or vice versa. These genes are picked up by the day 3-5 and 5-9 time frames. The number of the genes that slowly change over time are shown between brackets in the day 3-9 time frame in Figure 8. Their number is roughly half of the total regulated genes from day 3-9. The Venn diagrams show for each time frame the number of genes that change in the same way for both systems, and the number of genes that change uniquely in a specific system and not in the other. From these comparisons, genes are selected that are only regulated in time in one system and never in the other system (unique for the system) as well as the genes that are always regulated in the same way in both systems (common for two systems). The numbers are given at the bottom of the figure. In this way differences in gene expression between the two systems due to time delays are filtered out. It can be seen from Figure 8 that:

(i) The amount of commonly regulated genes is about twice the uniquely regulated genes for each system except for the up-regulated genes in ambr from day 5-9 where it is more or less comparable.

(ii) From day 3-5 the amount of uniquely regulated genes is the same between the two systems whereas from day 5-9 it is twice as much in the ambr as compared to the $10 \mathrm{~L}$ system. This is in agreement with the observation in the PCA analysis that the difference between both systems becomes progressively more with time. This may be related to the larger fluctuations in conditions, like the $\mathrm{pH}, \mathrm{DO}$, and nutrient concentrations in the ambr system.

(iii) The total amount of uniquely regulated genes in two systems is 4059 (total number of genes in "unique for each system" in Figure 8), which is in the same range as the total amount of common genes in two systems (5304, "common for two systems" in Figure 8). The total number of uniquely regulated genes is higher in the ambr than in the 10L reactor, which again may be due to the higher fluctuations in conditions like $\mathrm{pH}, \mathrm{DO}$, and nutrient concentrations. 


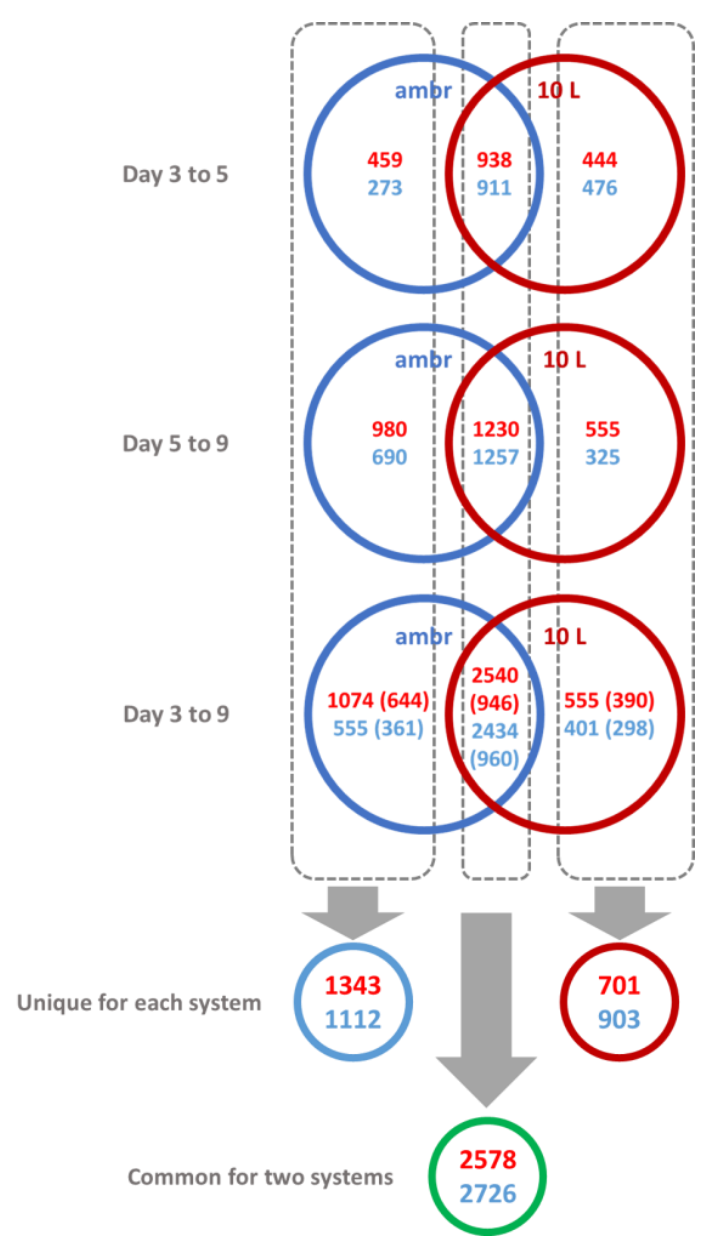

Figure 8. Venn diagram of the number of genes that are differentially regulated from day 3 to 5 , 5 to 9, and 3 to 9 for the ambr and 10 L cultures. Red numbers indicate up-regulation, blue numbers indicate downregulation. Numbers in brackets from day 3 to 9 show the number of genes significantly regulated from day 3 to 9, but not from day 3 to 5 and day 5 to 9. The number of regulated genes that are unique to each system, and are common for two systems (dash boxes) are also shown. A gene is unique to a system means it is differentially expressed in at least one of the three time comparisons for that system and never differentially expressed in one of the three time comparisons for the other system.

The biological function of the identified genes that were uniquely regulated in one of the systems (the genes in "unique for each system" in Figure 8) are next categorized based on the KEGG pathway database. The aim is to study whether these genes specifically belong to certain pathways that can be linked to the differences that exist between the scales.

The genes unique to a system are distributed over almost all the functional pathways, including global metabolism of carbohydrate, protein, lipid, nucleotide, energy, signal transduction, etc. In the ambr cultures, overexpression of the hypoxiainducible factor 1 (HIF-1) signaling pathways (Supplementary $\mathbf{C}$ to be published in the online version) is observed. HIF-1 is known to respond to extracellular oxygen levels and to play a role in anaerobic respiration and lactate formation ${ }^{180}$. The higher regulation of the HIF-1 signaling pathways in ambr may be caused by the fluctuations in DO (Figure 1) and may be related to the higher lactate production 
(Figure 5A). Moreover, as mentioned before, higher $\mathrm{pH}$ levels from day 4 to 8 and higher $\mathrm{pH}$ spikes towards higher values are observed in the ambr cultures (Figure 1D\&E), which would favor the transport of lactate out of cells. The transport is done by $\mathrm{H}^{+}$-monocarboxylate cotransporters (MCTs) ${ }^{181}$. Among several MCTs (MCT1MCT4) that are known for lactate transport, MCT2 (encoded by SLC16A7 gene) has a higher affinity for substrates compared to the other $\mathrm{MCTs}^{182}$ and it showed a higher up-regulation from day 3-9 in the $10 \mathrm{~L}$ cultures (3.6 FC, FDR.BH=0.00) compared to that of in the ambr cultures $(2.2 \mathrm{FC}, \mathrm{FDR} . \mathrm{BH}=0.00)$ (to be published in the Supplementary A of the online version). This might be associated with the differences in lactate production/consumption between the two systems (Figure 5A). Regulations of MCT1, MCT3, and MCT4 are however similar between the two systems. Furthermore, for the N-glycan biosynthesis pathway (to be published in the Supplementary $\mathrm{C}$ of the online version), the one up-regulated gene unique to the 10 L system is Fut8 (1.5 FC from day 3-9, FDR.BH=0.00) which gene encodes the fucosyltransferase that is responsible for the $\alpha-1,6$-fucosylation. This agrees with the observation of the higher percentage of fucosylated $\mathrm{mAb}$ in the $10 \mathrm{~L}$ cultures (Figure 3C).

In summary, only for a few genes a possible relationship between their expression and differences that exist between both scales can be found, which could be related to the fact that the differences between both systems in terms of process performance are small. Given this fact, the fraction of uniquely regulated genes of $20 \%$ is rather high (4059 uniquely regulated genes out of a total of 20858, Figure 8). Possibly the difference in gene expression of these genes between both scales represents an adaptive response to differences that exist between scales, leading to a comparable process performance. Overall, this study shows that transcriptomics is a sensitive tool to measure differences in the physiological response of cells to different bioreactor environments. However, these differences in gene expression could not be related to the process performance, which was comparable between scales or differences in the bioreactor systems. To be able to rate the importance of the observed differences, comparison is needed on gene expression data of other scale comparisons from both successful and failed scale-up/scale down experiments. These data are currently lacking in literature.

\subsection{Conclusion}

For the specific cell line and the fed-batch process tested in this study, the results obtained in the ambr at two different agitation rates are comparable to that obtained in a $10 \mathrm{~L}$ bioreactor in terms of cell growth, metabolism, productivity, and product quality. Transcriptome analysis showed differences in gene expression between both systems. The number of genes that were significantly regulated over culture time in only one of the systems and never in the other was higher in the ambr (2455) than in the 10 L system (1604), which may be related to the higher fluctuation of conditions 
like $\mathrm{DO}, \mathrm{pH}$ and nutrient concentrations in the ambr. In general, these genes were spread over all KEGG pathways and could not be linked to differences in bioreactor system or differences in cell physiology. For a few genes possible functional relationships with differences in $\mathrm{DO}$ and $\mathrm{pH}$ patterns, lactate metabolism, and glycosylation pattern could be identified. Furthermore, transcriptome analysis showed that a difference in gene expression between the inocula was not present anymore after three days of culture, and thus did not influence the system comparison. In addition to existing literature, this study further strengthens the opinion that the ambr system gives representative culture performances for the $10 \mathrm{~L}$ bench-scale bioreactor. Differences in gene expression between scales could be observed by using transcriptome analysis. However, the differences could not be linked to specific process conditions. More studies are needed to determine the sensitivity of the transcriptome analysis on scale comparison. 


Chapter 6

General discussion 


\begin{abstract}
:
Bioprocess scale-up is an important step during the upstream development of mammalian cell culture processes. Considerable progress has been made to understand the principles of process scale-up as well as to develop representative scale-down bioreactor systems. In this chapter, the current opinion on cell culture process scale-up is reviewed. Furthermore, with the increasing pressure on shortening the development timelines for biopharmaceutical manufacturing, miniature bioreactor systems which offer the high-throughput solution have received a lot of attention in recent years. These miniature bioreactor systems are being further incorporated into process development as scale-down models for the traditional bench-scale $(10 \mathrm{~L})$ bioreactors for the exploration of the process design space. The development of miniature bioreactor systems brings opportunities as well as challenges to the current process development. These are discussed in this chapter.
\end{abstract}




\subsection{Introduction}

Monoclonal antibodies (mAbs) are an important class of biopharmaceutical products ${ }^{2}$. Currently, mAbs are mainly manufactured in mammalian cell cultures in bioreactors. Chinese Hamster Ovary $(\mathrm{CHO})$ cells are the widely used hosts for the production of mAbs. In industry, a $\mathrm{CHO}$ cell culture process is typically developed at laboratory scale $(<20 \mathrm{~L})$ and scaled up to large scale (e.g. kL-scale) for commercial production ${ }^{183}$. The scale-up is done stepwise: from bench scale $(1 \mathrm{~L}$ to $20 \mathrm{~L}$ ) to pilot scale (500 L to $1000 \mathrm{~L}$ ) and finally to production scale ( $>2000 \mathrm{~L})$. The aim of scale-up is to increase the batch size while maintaining the same process performance. An important aspect of the process performance is product quality. During process scale-up, critical quality attributes (CQAs, e.g. oxidation, deamination, glycosylation, and aggregate levels) which influences the efficacy and safety of the product should be kept within acceptable ranges. In addition, key performance indicators for the process (KPIs, e.g. product concentration, cell growth, and viability) that reflect the performance of the cells need to be kept similar as well between scales ${ }^{184}$. The chemical (e.g. nutrient and waste concentrations, $\mathrm{pH}$, dissolved oxygen, dissolved carbon dioxide) and physical (shear) conditions experienced by the cells are important factors to consider during scale-up. These conditions depend on a combination of cell kinetics, liquid-gas mass transfer and mixing, which together determine the nutrient and product concentrations as well as whether significant gradients will be present in the bioreactor. In bioreactors, mass transfer, mixing, and shear can be linked to a number of parameters, for example, volumetric energy input $(\mathrm{P} / \mathrm{V})$, impeller tip speed ( $\mathrm{Vtip}$ ), impeller pump rate $(\mathrm{Q})$, and Reynolds number $(\mathrm{Re})^{185}$. However, it is not possible to keep all these parameters the same across scales. Consequently, keeping mass transfer between scales the same will result in different shear conditions. Hence, it is important to understand the interactions between these parameters and whether they affect the cell and process performance in the applied ranges for the different scales. Based on this, choices can be made on which parameters are important to be kept constant across scales. Although a better understanding has been obtained on how to keep the scaling parameters within acceptable ranges during the past decades ${ }^{37-39}$, scale-up is still not a straightforward and uniform task. It remains a critical and challenging step in mammalian cell process development especially due to the fact that the scales used in development are getting smaller. 


\begin{tabular}{|c|c|c|c|c|c|c|}
\hline $\begin{array}{l}\text { Bioreactor } \\
\text { systems }\end{array}$ & $\begin{array}{c}\text { Working } \\
\text { volume }(\mathrm{mL})\end{array}$ & $\begin{array}{l}\text { Multi } \\
\text { plex }\end{array}$ & Feed addition & Aeration & $\begin{array}{l}\text { Singl } \\
\text { e-use }\end{array}$ & Reference \\
\hline $\begin{array}{l}\text { Applikon } \\
\text { Mini Bio }\end{array}$ & $50-800$ & $2-8$ & $\begin{array}{l}\text { Continuous feeding } \\
\text { possible }\end{array}$ & $\begin{array}{c}\text { Sparging/Overlay } \\
\text { Porous sparger /L-type } \\
\text { sparger }\end{array}$ & $\mathrm{N}$ & $\begin{array}{c}\text { http://www.applikon- } \\
\text { bio.com/ } \\
186\end{array}$ \\
\hline $\mathrm{ambr}^{\circledR} 15$ & $10-15$ & $24-48$ & Bolus feeding & $\begin{array}{l}\text { Sparging/Overlay } \\
\text { Open pipe sparger }\end{array}$ & $\mathrm{Y}$ & $\begin{array}{c}\text { WWW.sartorius.com } \\
174\end{array}$ \\
\hline $\mathrm{ambr}^{\circledR} 250$ & $100-250$ & $12-24$ & $\begin{array}{l}\text { Continuous feeding } \\
\text { possible }\end{array}$ & Sparging/Overlay & $\mathrm{Y}$ & $\begin{array}{c}\text { Www.sartorius.com } \\
33\end{array}$ \\
\hline DASbox $^{\circledR}$ & $60-250$ & 4/Unit & $\begin{array}{l}\text { Continuous feeding } \\
\text { possible }\end{array}$ & Sparging/Overlay & $\mathrm{N}$ & $\begin{array}{l}\text { Www.eppendorf.com } \\
187\end{array}$ \\
\hline BioXplorer & $100-400$ & $\begin{array}{c}\text { 4- } \\
\text { 8/Unit }\end{array}$ & $\begin{array}{l}\text { Continuous feeding } \\
\text { possible }\end{array}$ & $\begin{array}{c}\text { Sparging } \\
\text { Open pipe sparger }\end{array}$ & $\mathrm{N}$ & $\begin{array}{c}\text { http://www.helgroup. } \\
\text { com/ } \\
166\end{array}$ \\
\hline MSBR & 10 & N/A & Bolus feeding & Sparging & $\mathrm{N}$ & $\begin{array}{c}\text { Not available } \\
\text { commercially } \\
188\end{array}$ \\
\hline
\end{tabular}

Bench-scale bioreactors (1-10 L) have been used as the main platform for process development and they were traditionally considered as the smallest scale that is still representative for the bioreactors at production scale. However, in order to obtain an accurate definition of the process design space, high throughput experimentation $(\mathrm{n}>100)$ is needed ${ }^{175,189}$. Using bench-scale bioreactors for high throughput experimentation is not practical due to the substantial cost associated with materials, space, time, and labor. Therefore, during the past few years, miniature bioreactor systems (mL-scale) have received a lot of attention in process development ${ }^{189}$. The larger number of experiments that can be done using a miniature bioreactor system as compared to the 1-10 L bench-scale bioreactors can expand the process knowledge space and help to define more accurately the process design/control space, which facilitates the quality by design $(\mathrm{QbD})$ approach. Several miniature systems at the mL-scale have been developed with similar control and operational capabilities as production-scale bioreactors (Table I). These capabilities include the monitoring and control of culture DO and $\mathrm{pH}$, mechanical mixing, gas sparging, and the addition of feed, antifoam, and base solutions. The mL-scale systems were originally designed for quick screening of clones, media, and culture parameters such as $\mathrm{pH}$ and temperature. In recent years, many studies $165,172,190,191$ have shown that these mL-scale systems are quite representative for the bioreactors ranging from L-scale to kL-scale. 
Data presented in Chapter 5 and in other studies ${ }^{34,169,170,172-174,192,193}$ showed that the current mL-scale systems are promising tools to be integrated into process characterization and design space exploration during upstream process development. Current research is focusing on the further implementation of mL-scale systems into process development and replacing the traditional bench-scale bioreactors with mLscale systems (Figure 1).

Nevertheless, challenges with regard to gas transfer, mixing, shear, and liquid handling still exist in the current mL-scale systems due to small culture volume and how these systems are operated. In this chapter, the current challenges in process scale-down to the $\mathrm{mL}$-scales are discussed in relation to the $\mathrm{ambr}^{\circledR} 15$ system as a model system.

\section{Lab/Bench scale}

\section{Pilot/Production scale}

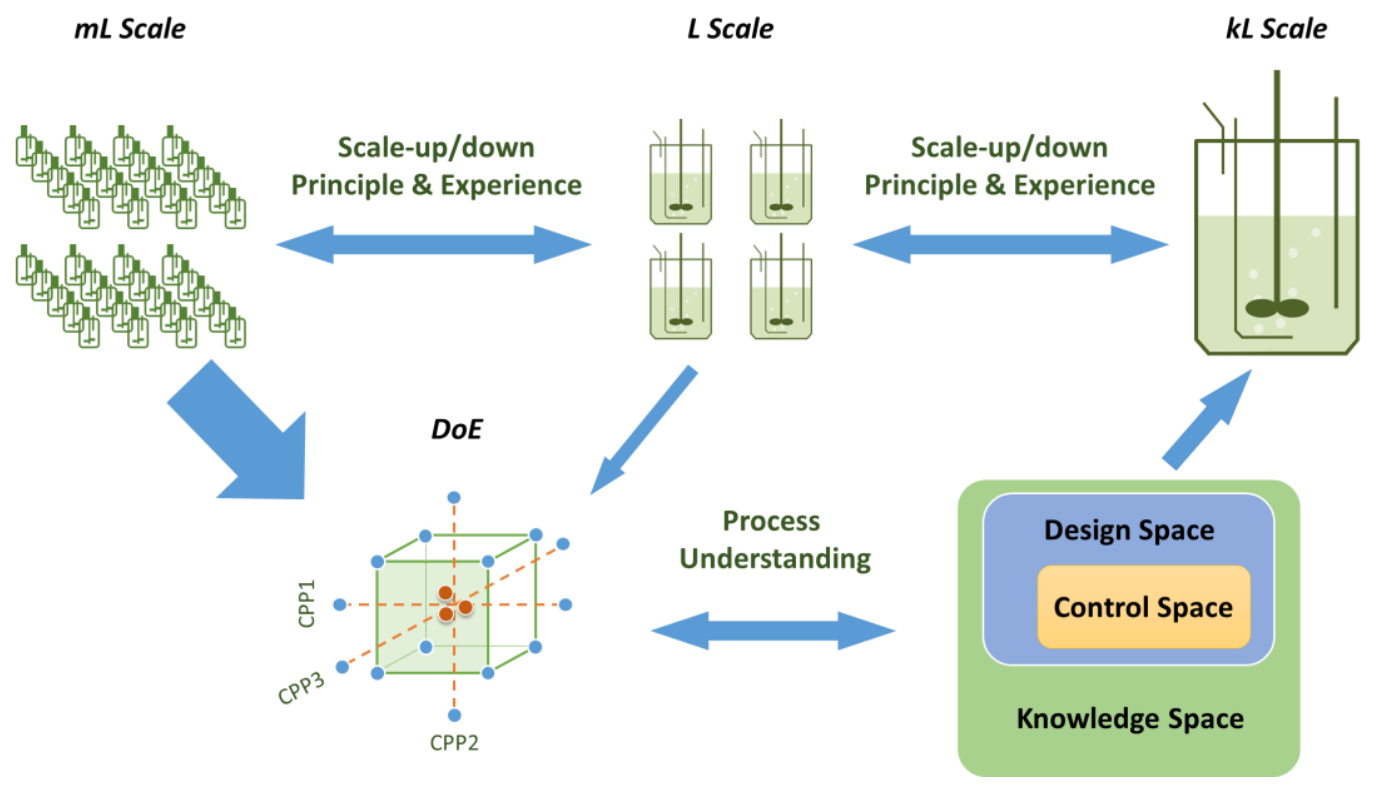

Figure 1. Scale-up/down during process development

\subsection{The scale-down approach}

In order to develop a representative small-scale bioreactor system, reversed scale-up as a scale-down approach (Figure 2) was proposed by Oosterhuis ${ }^{194}$. As the first step of the scale-down approach, it should be identified which are the parameters that can be kept the same between scales and which cannot. Next, for the parameters that cannot be easily kept the same between scales, their effects on the cells and the process performance have to be analyzed ${ }^{195,196}$ in order to make sure that they are kept in ranges where they do not affect the process outcome. Based on this information, choices can be made on how to perform scale-down. For mammalian 
cell culture the three aspects that cannot all be kept the same between scales and for which the importance for process performance has to be studied are:

> Gas-liquid mass transfer, which determines the supply of $\mathrm{O}_{2}$ and removal of $\mathrm{CO}_{2}$.

Mixing, which is used to homogenize the culture and is related to gradients in the reactor.

Shear effects, which are the result of sparging and agitation and thus are associated with the previous two aspects.

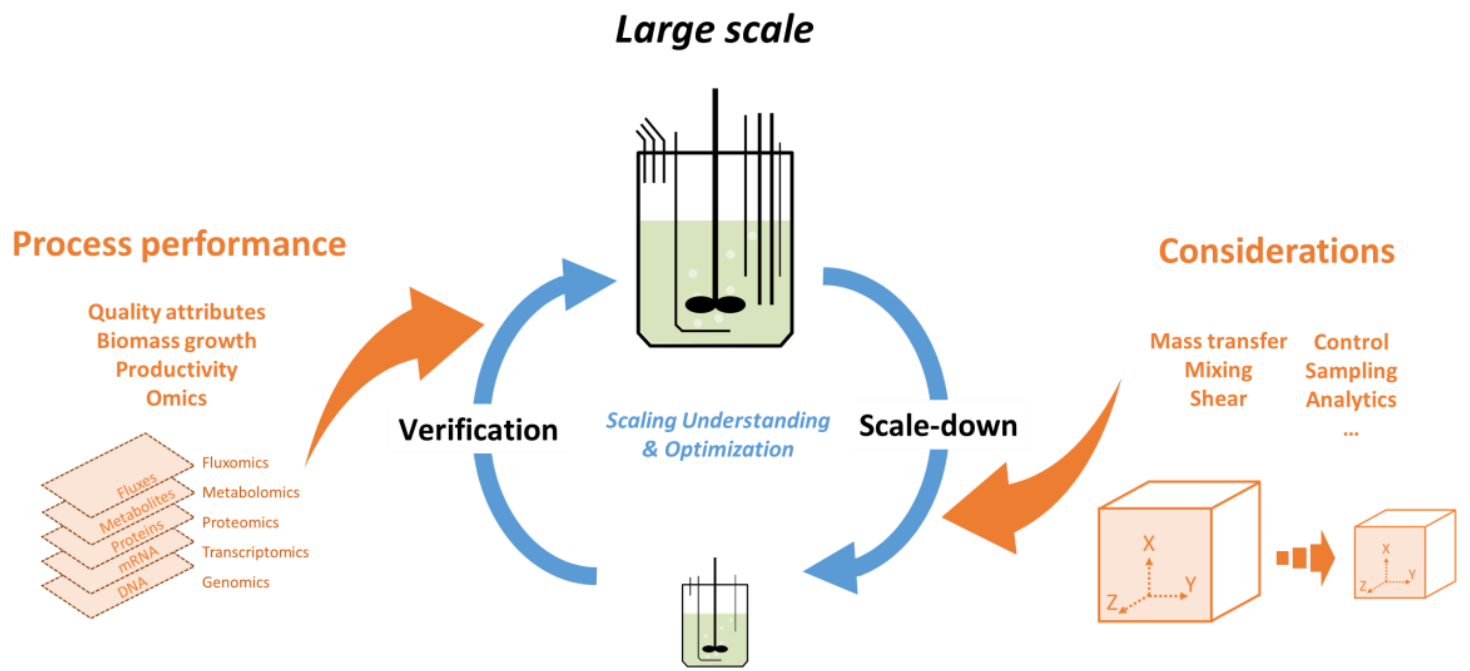

Small scale

Figure 2. The general scale-down approach

The performance of the scaled-down process with regards to quality attributes, productivity, etc. needs to be validated against the production-scale reactor. If a deviation is observed, further optimization of the scaled-down process will be needed (Figure 2).

\subsubsection{Mass transfer}

Dissolved oxygen (DO) and dissolved carbon dioxide $\left(\mathrm{pCO}_{2}\right)$ concentrations are critical parameters for mammalian cell culture performance. Insufficient $\mathrm{O}_{2}$ transfer results in $\mathrm{O}_{2}$ limitation, which leads to slower cell growth, changes in cell metabolism, cell death, and changes in product quality ${ }^{179,197}$. In practice, the $\mathrm{O}_{2}$ volumetric mass

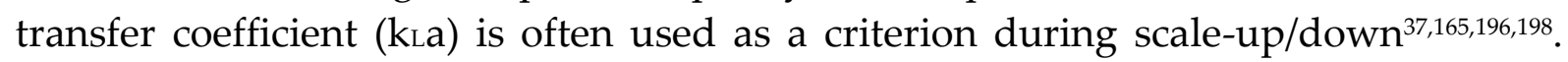
Matching the kta ensures that similar maximum $\mathrm{O}_{2}$ transfer rates (OTRs) can be obtained between scales. 
$\mathrm{CO}_{2}$ is a by-product of mammalian cell metabolism and is produced at roughly the same rate as with which $\mathrm{O}_{2}$ is consumed ${ }^{199}$. High $\mathrm{pCO}_{2}$ values $(>150 \mathrm{mmHg}$ partial pressure, or $>4.5 \mathrm{mM}$ concentration) were reported to negatively influence cell growth, productivity, and protein quality ${ }^{20,42,200,201}$. Mostafa and $\mathrm{Gu}^{202}$ reported a $40 \%$ loss of specific productivity in $\mathrm{CHO}$ cell culture when the $\mathrm{pCO}_{2}$ increased from 68 $\mathrm{mmHg}$ in a bench-scale $(1.5 \mathrm{~L}) \mathrm{STR}$ to $179 \mathrm{mmHg}$ in a pilot-scale $(1000 \mathrm{~L})$ STR. In order to maintain a similar $\mathrm{pCO}_{2}$ profile between scales, it is important to have a similar volumetric $\mathrm{CO}_{2}$ removal rate in bioreactors of different volumes.

Aeration using micro-bubbles (e.g. $\left.\mathrm{d}_{b}<200 \mu \mathrm{m}\right)$ with pure $\mathrm{O}_{2}$ is favorable for $\mathrm{O}_{2}$ transfer and is often applied at bench and manufacturing scales. The use of pure $\mathrm{O}_{2}$ increases the driving force for $\mathrm{O}_{2}$ transfer, while the smaller bubble size and the slower rising velocity of these micro-bubbles result in a high specific surface area of bubbles and thus a high $\mathrm{O}_{2}$ volumetric mass transfer coefficient $(\mathrm{kLa})^{22,203-206}$. As a consequence, higher OTRs can be achieved and a significantly lower volumetric gas flow rate is needed. Furthermore, some of the small bubbles collapse and thus never reach the surface ${ }^{207}$, which in combination with the lower volumetric gas flow rate reduce problems with foaming and risks with bubble-burst associated cell damage. However, the use of micro-sparging and pure $\mathrm{O}_{2}$ results in high $\mathrm{pCO}_{2}$ levels ${ }^{43,203,204,208}$. When micro-bubbles with pure $\mathrm{O}_{2}$ are used, the volumetric gas flow rate that is required for $\mathrm{O}_{2}$ transfer is considerably lower compared to when large bubbles are used. At a lower volumetric gas flow rate in order to reach the same volumetric $\mathrm{CO}_{2}$ removal rate, the $\mathrm{CO}_{2}$ concentration in the gas has to be higher. This means that the concentration of $\mathrm{CO}_{2}$ in the liquid phase must also be higher to have a concentration gradient required for diffusion from the medium to the bubble. Therefore, the $\mathrm{pCO}_{2}$ level will build up with the use of micro-sparging. In order to reduce the $\mathrm{pCO}_{2}$ level in these bioreactors, an additional sparger generating large bubbles with air or $\mathrm{N}_{2}$ is used for stripping $\mathrm{CO}_{2}{ }^{165,209}$. In this way, the $\mathrm{DO}$ and $\mathrm{pCO}_{2}$ can be independently controlled.

An obvious difference between scales is that the contribution of the headspace surface to gas transfer becomes relatively more important upon scale-down, because the volume decreases more rapidly than the headspace surface and thus the specific surface area increases. At $10 \mathrm{~L}$ scale, the contribution of the headspace surface to gas transfer is still negligible. However, in the $\operatorname{ambr}^{\circledR} 15$ bioreactors according to Nienow et al. ${ }^{168}$, the apparent kLa from the headspace surface aeration can contribute to more than $50 \%$ of the total apparent kLa. The difference in the contribution of the headspace surface to gas transfer results in different gassing strategies and $\mathrm{pCO}_{2}$ between scales. For example, because of the high gas transfer from the headspace surface in the ambr ${ }^{\circledR} 15$ bioreactors, micro-sparging is not needed and only large sparging bubbles are used in the ambr ${ }^{\circledR} 15$ bioreactors (Chapter 5). As a consequence, the volumetric gas flow rate in the $\mathrm{ambr}^{\circledR} 15$ bioreactor is significantly higher (e.g. 2 to 9 times higher in Chapter 5) than in the $10 \mathrm{~L}$ scale bioreactors. Subsequently, lower 
$\mathrm{pCO}_{2}$ levels were observed in the $\mathrm{ambr}^{\circledR} 15$ bioreactors compared to in the $10 \mathrm{~L}$ bioreactors (Chapter 5). A way to improve this is to use micro-sparging for DO control in the $\operatorname{ambr}^{\circledR} 15$, similar to those used in bench scales, and to use headspace aeration for $\mathrm{pCO}_{2}$ control, for example, by gassing air through the headspace enriched with $\mathrm{N}_{2}$ or $\mathrm{CO}_{2}$ depending on the oxygen transfer and culture $\mathrm{pCO}_{2}$, respectively.

The higher contribution by surface aeration also means that a small change in culture volume in the mL-scale bioreactors due to, for example, sampling or feed addition will result in more significant changes in the kia due to the change in the headspace specific surface area. Nienow et al. ${ }^{168}$ showed that a $2 \mathrm{~mL}$ volume decrease from 15 to $13 \mathrm{~mL}$ in the $\mathrm{ambr}^{\circledR} 15$ bioreactor results in a 20 60\% increase in the apparent kLa. This might lead to a change in gas flow rate and difficulties in controlling the DO and $\mathrm{pCO}_{2}$, which could influence the process performance at the mL-scale. Finally, in the $\mathrm{ambr}^{\circledR} 15$ system, the lid is opened for sampling and feed/base addition. This causes a sudden change in gas composition in headspace, which will also introduce temporary changes in $\mathrm{DO}$ and $\mathrm{pCO}_{2}$ (Chapter 5).

In order to better simulate the $\mathrm{pCO}_{2}$ condition in the large-scale bioreactors, as mentioned before, it may be necessary to increase the $\mathrm{pCO}_{2}$ in $\mathrm{mL}$-scale bioreactors by enriching the inlet gas with $\mathrm{CO}_{2}$. Although several studies have attempted this simulation using scale-down bioreactors, to precisely reproduce the $\mathrm{pCO}_{2}$ levels between scales remains a difficult task since by manipulating the $\mathrm{pCO}_{2}$ level the gassing strategy, $\mathrm{pH}$ control, and osmolality will be influenced ${ }^{20,42,200,201}$. Ideally, in the mL-scale bioreactors, micro-sparging should be used to simulate the shear conditions as in the large-scale bioreactors, and macro-sparging or headspace aeration should be used to control the $\mathrm{pCO}_{2}$ levels. This will be a technical challenge for the systems with extremely small volumes (e.g. ambr ${ }^{\circledR} 15$ system), however, it may easily be implemented, for example, in the $\operatorname{ambr}^{\circledR} 250$ system. These modifications will only be necessary if the shear caused by micro-bubbles (see 2.3) and the $\mathrm{pCO}_{2}$ level are critical for the process performance.

\subsubsection{Mixing}

Sufficient mixing in a bioreactor is needed to maintain homogeneous conditions for the cells. Mixing time ( $\mathrm{T}_{\mathrm{m}}=$ time to reach $95 \%$ homogeneity after a local change) is used to evaluate the mixing characteristics of a bioprocess ${ }^{185,210}$. $\mathrm{T}_{\mathrm{m}}$ tends to be longer in large-scale bioreactors than in small-scale bioreactors ${ }^{22,207}$, meaning that in larger bioreactors a cell travels through regions with different environments (gradients of $\mathrm{pH}, \mathrm{DO}$, nutrient concentration, etc.) for a longer time ${ }^{195}$. In manufacturing-scale bioreactors, the mixing time is generally in the range of minutes ${ }^{185}$. In mL-scale bioreactors, mixing times are much shorter (e.g. according to Nienow et al. ${ }^{168}$, the typical $\mathrm{T}_{\mathrm{m}}$ for the $\mathrm{ambr}^{\circledR} 15$ bioreactors is below $\left.10 \mathrm{~s}\right)$. This may result in a different 
cell performance compared to large-scale reactors if the longer mixing time in these large-scale reactors causes gradients that affect cell physiology. Gradients may be caused by the local addition of feeds and base or the metabolic activity of the cells (e.g. local consumption/depletion of $\mathrm{O}_{2}$ ). In the case of local addition of feeds and base, sudden changes in local $\mathrm{pH}, \mathrm{DO}$, osmolality, and nutrients concentration will occur ${ }^{195}$. These changes are steep but last only for short periods of time. The $\mathrm{T}_{\mathrm{m}}$ determines how long the gradients will be present and how steep these gradients are. The influence of such gradients on cells will also be dependent upon the sensitivity of the cells, which needs to be determined experimentally. Gradients caused by the metabolic activity of the cells will in general be less steep but persist for longer periods of times. If the mixing time $\mathrm{T}_{\mathrm{m}}$ is larger than the cell characteristic time for the uptake of nutrients or production of waste products, gradients for these components are likely to be present, and cell metabolism may be affected, again, depending on sensitivity of the cell ${ }^{195,211}$. Oxygen is one of the most critical components since it has a low solubility and is consumed by cells at a high rate and is thus most likely the first component to be locally depleted ${ }^{212}$. The characteristic time for oxygen uptake depends on the cell concentration and the specific oxygen uptake rate, which depend on the cell line and the conditions used. Typical values for a $\mathrm{CHO}$ cell fed-batch process are a viable cell concentration of $2 \times 10^{7}$ cells $/ \mathrm{mL}$ and a specific $\mathrm{O}_{2}$ uptake rate of $10^{-16} \mathrm{~mol} / \mathrm{cell} / \mathrm{s}$, which results in a characteristic time for $\mathrm{O}_{2}$ uptake of $\sim 100 \mathrm{~s}^{185}$. For perfusion cultures, which reach cell densities about 5 times as high, the characteristic time becomes $\sim 20 \mathrm{~s}$.

Practically, a maximum allowed mixing time is applied in industry for designing operating conditions of large-scale bioreactors. This value can be determined experimentally based on, for example, the characteristic time for $\mathrm{O}_{2}$ uptake. If the mixing time is kept below this threshold, it is assumed that the gradients do not affect the performance of the process. For large-scale STRs, sufficient mixing is ensured by adding baffles and extra impellers, and by intensifying agitation. However, elevated shear forces are associated with these options (see section 2.3). As a result, limits in either mixing time or shear may be reached as the scale increases ${ }^{43}$.

Several studies have attempted to simulate the mixing conditions in large-scale bioreactors using scaled-down bioreactors. In the study of Nienow et al. ${ }^{171}$, an external plug-flow reactor (PFR) connected to a 3-L STR through a peristaltic pump has been used to simulate the impact of spatial variations in $\mathrm{pH}$ and nutrients as found at commercial scale. Substrate and alkali with different $\mathrm{pH}$ (9.7 and 12.3) were fed to the STR or the PFR under different circulation rates (60 s and $120 \mathrm{~s})$. However, the use of the peristaltic pump in that study always resulted in reductions in culture time and product titer, which was dominant enough to mask other effects. In contrast, a one-compartment study was performed by Serrato et al. ${ }^{179}$ in a $220-\mathrm{mL}$ bioreactor to simulate DO oscillations in large-scale hybridoma cultures producing a mAb. The study showed that compared to a constant DO $(10 \%)$, the oscillating DO $(0 \%-14 \%)$ in 
a time-scale ranging from 800 to $12800 \mathrm{~s}$ decreased maximum cell concentrations, cell growth rates, and viability, and increased cell glycolytic metabolism and lactate production. Furthermore, the oscillating DO resulted in a higher amount of triantennary and sialylated glycans, which may affect the quality of the mAb. Recently, Brunner, M. et al. ${ }^{213}$ studied the impact of $\mathrm{pH}$ inhomogeneity on $\mathrm{CHO}$ cell fed-batch process performance using a two-compartment (a 3 L STR and a 0.7 L STR) scale-down modelling. Their study simulated a well-mixed zone of pH 7.0 in the $3 \mathrm{~L}$ STR and a temporary $\mathrm{pH}$ zone of $\mathrm{pH} 9.0$ in the $0.7 \mathrm{~L}$ STR, connected by recirculation peristaltic pumps. The study showed that the temporary $\mathrm{pH}$ zone has a strong impact on maximum viable cell density and final product titer. Unlike the previous study done by Nienow et al. ${ }^{171}$, in the study of Brunner, M. et al. ${ }^{213}$, the use of peristaltic pumps did not show an effect on performance of the applied cell line. In conclusion, only a few experiments have been performed to understand the effects of gradient and poor mixing conditions in mammalian cell culture bioreactors. There is still a lot to be done to study the effect of gradients on relevant conditions and timescales for the factors like $\mathrm{pH}, \mathrm{DO}$, and individual nutrients.

\subsubsection{Shear}

As mentioned before, in large-scale bioreactors, the chosen operating parameters for mixing (e.g. stirrer types and agitation speeds) and mass transfer (e.g. sparging bubble sizes and rates) will have an impact on the shear level. If shear affects the process performance, it is important that the shear level is comparable between largeand small-scale systems so that the potential problems related to this can be identified early at the small-scale systems.

The most obvious and often considered source of shear stress is agitation. Research has been done to investigate maximum allowed power input per volume $(\mathrm{W} / \mathrm{kg}$, also called volumetric energy dissipation rate, from here on symbolized as $[\varepsilon]$ ) for mammalian cells $37,38,165,177,196,214$. The results of these studies indicate that shear generated from agitation is generally well below the critical level of mammalian cells. In typical large-scale bioreactors, the average $[\varepsilon]$ is around $0.01 \mathrm{~W} / \mathrm{kg}$ and the maximum $[\varepsilon]$ from agitation would be in the order of $1 \mathrm{~W} / \mathrm{kg}{ }^{207}$. Nienow et al. ${ }^{171}$ reported that the cell growth and productivity remained unchanged at significantly higher $[\varepsilon]$ values, i.e. $290 \mathrm{~W} / \mathrm{kg}$, created by an extensional flow device, or an average

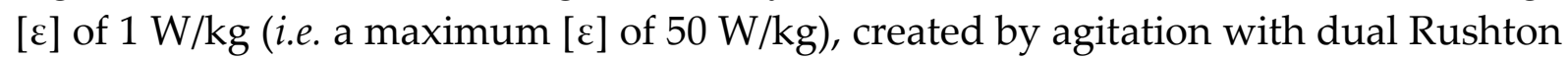
turbines. In the studies of Godoy-Silva et al. ${ }^{215,216}$ and Ma et al. ${ }^{217}$, mammalian cells were subjected to very high shear stresses in external "torturing chambers". Changes with respect to cell growth, viability, and antibody glycosylation were found only at [ع] values (e.g. $\sim 290 \mathrm{~W} / \mathrm{kg}$ ) that were orders of magnitude higher than the estimated maximum $[\varepsilon]$ generated from agitation in large-scale STRs. In contrast, when an agitation rate condition of $700 \mathrm{RPM}(\sim 0.5 \mathrm{~W} / \mathrm{kg})$ was applied in a 4 L bioreactor 
experiment done by Sieck et al. ${ }^{32}$, reduced culture viable cell density and viability were observed compared to a standard agitation rate condition (267 RPM, $\sim 0.03$ $\mathrm{W} / \mathrm{kg}$ ). This stress level at which damage occurs is thus substantially lower than those reported by others. Although not mentioned in that study, bubble entrainment could have been the cause of the cell damage. It can also be that the specific cell line used is very sensitive to stress.

In industry, an appropriate agitation scale-up/down criterion is chosen between scales to ensure similar mixing intensities, and for some shear sensitive cell lines, also similar shear levels ${ }^{169,218}$. The aforementioned $[\varepsilon]$, which is also referred to as the $\mathrm{P} / \mathrm{V}$ value, is often used as the criterion. Li et al. ${ }^{37}$ did a scale-down from a $2000 \mathrm{~L}$ to a $2 \mathrm{~L}$ STR based on a constant impeller tip speed, resulting in 550 RPM in the 2 L STR, and based on a constant impeller P/V value, resulting in $350 \mathrm{RPM}$ in the $2 \mathrm{~L}$ STR. The $2 \mathrm{~L}$ cultures based on the $\mathrm{P} / \mathrm{V}$ value gave a representative performance, whereas the $2 \mathrm{~L}$ cultures based on the constant impeller tip speed showed a lower cell density and viability. Also here it could be that the high RPM led to vortex formation that caused bubble entrainment ${ }^{40}$, which in turn caused cell damage. In Chapter 5 of this thesis, two agitation scale-down approaches were tested in the $\operatorname{ambr}^{\circledR} 15$ compared to a $10 \mathrm{~L}$ bioreactor system: 800 RPM based on matching impeller tip speed and 1300 RPM based on matching impeller specific power input $\mathrm{P} / \mathrm{V}$. No difference in process performance was found between both approaches. This can be expected due to the fact that the $\mathrm{P} / \mathrm{V}$ values of both approaches $(0.065 \mathrm{~W} / \mathrm{kg}$ and $0.28 \mathrm{~W} / \mathrm{kg}$, respectively) were far below the aforementioned threshold that would influence the process performance. Although agitation is an obvious source of shear, current studies suggested that it is not a major limitation for large-scale mammalian cell culture anymore and thus is not important for scale-down, as long as there is no bubble entrainment from a vortex.

In addition to agitation, shear can also be caused by the interaction between cells and bubbles. In a STR, bubble-associated cell damage may occur in three main regions: (i) at the sparger due to bubble generation, (ii) in the bulk of the liquid due to rising of the bubbles, and (iii) at the surface due to bubble bursting.

Within these three regions where cell damage may occur, generally, it is believed that the (ii) rising of the bubbles has little effect on cell damage ${ }^{207}$. However, the (iii) bubble bursting is considered as the most important cause of cell death ${ }^{219-222}$. Studies have shown that upon bubble rupture, substantial energy $\left(\sim 1 \times 10^{5} \mathrm{~W} / \mathrm{kg}\right)$ is discharged to the surrounding liquid which damages the cells ${ }^{11,21,223}$. More importantly, the $[\varepsilon]$ associated with the bubble burst is inversely correlated to bubble $\operatorname{size}^{21,221,224}$, i.e. the burst of smaller bubbles is more detrimental to cells. Currently, shear protective reagents such as the Pluronic F-68 have largely solved the problem related to the bubble damage ${ }^{225-227}$. Several possible protective mechanisms of Pluronic F-68 have been proposed. One is that it forms a protective layer on the cell membrane which increases the shear resistance of the cells ${ }^{227}$. Another explanation is 
that it saturates the air-liquid interface and therefore displacing cells ${ }^{226}$. In addition, Pluronic F-68 may also decrease surface tension and thus decrease the energy generated from bubble bursting ${ }^{228}$. Increase in Pluronic F-68 up to $2 \mathrm{~g} / \mathrm{L}$ generally showed increasing protection effect for $\mathrm{CHO}$ and insect cells against shear damage 225,227,229.

Furthermore, some studies focused on cell damage in the (i) bubble generation region. Barbosa et al. ${ }^{230}$ showed that at high gas entrance velocities $(52 \mathrm{~m} / \mathrm{s})$ death of algal cells may occur at the sparger site. Similar to the result of Barbosa et al. ${ }^{230}$, Zhu et al. ${ }^{231}$ studied the influence of sparging on a mammalian (NS0) cell line in $8 \mathrm{~L}$ spinners. It was shown in that study that a gas entrance velocity above $30 \mathrm{~m} / \mathrm{s}$ resulted in cell death at the sparger site and the cell death was mainly a function of the gas entrance velocity with minimum contributions of total gas flow rate and bubble burst $\left(\mathrm{d}_{\mathrm{b}}=6-20 \mathrm{~mm}\right)$. Liu et al. ${ }^{232}$ developed a hydrodynamic model which showed a good correlation with experimental data indicating that the increased gas flow rate enhances cell damage at the gas inlet region near the sparger. These studies indicate that cell damage may occur in the bubble formation region. Practically, gas entrance velocities can be easily kept below $30 \mathrm{~m} / \mathrm{s}$ if the spargers are properly designed, meaning cell death at the sparger is not a major problem in scale up/down.

Alternatively, the cell death from cell-bubble interaction can be determined using the hypothetical killing volume model. The model relates the first order death rate constant $\mathrm{kd}_{\mathrm{d}}\left(\mathrm{s}^{-1}\right)$ to reactor parameters:

$$
k_{d}=\frac{6 F V_{K}}{\pi d_{b}^{3} V}
$$

where $\mathrm{F}$ is the gas flow rate $\left(\mathrm{m}^{3} \times \mathrm{S}^{-1}\right) \mathrm{V}_{\mathrm{k}}$ is the hypothetical killing volume $(-), \mathrm{d}_{\mathrm{b}}$ is the bubble diameter $(\mathrm{m})$ and $\mathrm{V}$ is the culture volume $\left(\mathrm{m}^{3}\right)$. The hypothetical killing volume $V_{k}$ is a scale-independent parameter, meaning differences between scales can be accounted for using this theory. However, the only problem is that $V_{k}$ is dependent on bubble diameter $\left(\mathrm{d}_{\mathrm{b}}\right)^{22}$ and the relation is not exactly known. According to Tramper et al. ${ }^{233,234}, \mathrm{~V}_{\mathrm{k}}$ is proportional to $d_{b}^{3}$. While in other studies, it was shown that the killing volume is proportional to $d_{b}^{21,222}$. This model was never verified for micro-sparging. In traditional low cell density $\left(<1 \times 10^{7}\right.$ cells $\left./ \mathrm{mL}\right)$ bioreactors often no micro-sparging is used and the use of Pluronic F-68 makes cell death at the surface negligible. In this circumstance, the differences in gassing will not be a problem in scale-down/up. However, the current mammalian cell culture is progressing towards high-density $\left(>5 \times 10^{7}\right.$ cells $\left./ \mathrm{mL}\right)$ processes (e.g. intensified fedbatch or perfusion cultures). In such cases, micro-sparging and higher sparging rates will be needed, which may bring back the issues associated with the bubble damage. In conclusion, the effect of bubble size on cell death needs to be studied in more detail. 


\subsubsection{Additional challenges of the current mL-scale systems}

Besides the challenges associated with the traditional scale-up/down problems, additional challenges exist with further scale-down to the mL-scale. These challenges are primarily associated with the small culture volume.

First, small volumes of liquid addition are needed for feeding and for $\mathrm{pH}$ control of the mL-scale bioreactor. In Chapter 5, the daily addition of feed medium for a $15 \mathrm{~mL}$ ambr $^{\circledR} 15$ bioreactor can be as low as $60 \mu \mathrm{L}$, which is not possible to be added accurately in a continuous way over 24 hours and thus can only be added as a bolus. The addition of feed as a bolus can lead to fluctuations in $\mathrm{pH}$ and $\mathrm{DO}$ profiles at the moment of addition (Chapter 5). Furthermore, bolus addition results in periodic variations in nutrient concentrations, whereas upon continuous addition, nutrient concentrations stay more or less constant. Thus, in case the continuous feeding is applied at large scale, the different feeding strategy applied at the mL-scale may cause changes in process performance such as cell metabolism and product quality. The further development of mL-scale systems requires liquid handling devices that can add liquid in small amounts continuously ${ }^{235}$.

Second, the sample volume is limited in the mL-scale bioreactor. A large sample volume withdrawal in a mL-scale bioreactor will disturb the kLa and DO. If the sample volume is larger than the feed volume, it will also result in a continuous decrease in culture volume and eventually emptying of the bioreactor. In order to solve this, in Chapter 5, destructive sampling for several ambr ${ }^{\circledR} 15$ bioreactors and pooling of the sample content from two ambr ${ }^{\circledR} 15$ bioreactors were used to achieve enough sample volume. The main problem with respect to this approach is that the number of experiments and the data output per experiment (i.e. the experimental power) are significantly reduced. Moreover, the size of the required sample is directly related to the volume required for the analytics. The solutions for decreasing the volumes required for analytics are further discussed in the next section.

\subsubsection{Scale-down verification}

Whether the small scale process is truly representative for the larger scale should be justified by experimental verification (Figure 2). For the process verification, ideally, the same analytics should be carried out between scales. However, as mentioned before, the sample volume that can be taken from a mL-scale bioreactor is much smaller than for a bench-scale or a production-scale bioreactor, which may limit the time resolution and the number of process parameters on which the scales are compared. To improve this, analytical methods with lower volume requirements have to be developed for mL-scale processes. A number of analytical methods that require much smaller volumes are being implemented into the $\mathrm{mL}$-scale bioreactors 
to solve this problem. The Octet ${ }^{\mathrm{TM}}$ system (Pall ForteBio Corp, USA) is able to perform protein quantitation and affinity analysis for $\mu \mathrm{L}$ sample volumes directly from culture supernatant, by employing advanced microfluidics and label-free assays. Miniature chromatography columns and antibody purification platforms have also been developed that can purify mAbs at a few milligrams level ${ }^{192,236-238}$. Several methods have enabled glycosylation analysis directly from culture supernatant and with a volume requirement in $\mu \mathrm{L}$ ranges ${ }^{239-242}$.

For the analytics of the mL-scale system, a second challenge arises due to the requirement of high experimental throughput. Large numbers of samples from the mL-scale system need to be analyzed and interpreted, which is a labor-intensive and time-consuming task at the moment. To tackle this, high throughput sample preparation, transfer, and analysis are necessary. Currently, instruments for online sampling, sample preparation, and transfer to analytical devices are being integrated into cell culture systems ${ }^{243}$. Several automation programming platforms have been developed that allow for cross-platform liquid handling using microfluidic devices $^{244,245}$. Such systems can drastically decrease the time requirement and labor intensity on the sampling actions of mL-scale systems. Advanced online cell culture monitoring technologies are available, including online dielectric spectroscopy ${ }^{246}$ and light scattering ${ }^{191}$, which can be used to measure viable cell density and viable cell volume, and online Raman spectroscopy ${ }^{247}$ and infrared spectroscopy ${ }^{248}$, which can be used to monitor several culture metabolites and glycoprotein titer. Integration of these technologies into mL-scale bioreactors can reduce the liquid handling activities, solve the problem related to the small sample volume, and more importantly, increase the data throughput and resolution.

Besides the routinely measured KPIs and CQAs, in this thesis, transcriptome analysis is used as a fingerprinting technique to evaluate the scale-down process. For the cell culture process run in Chapter 5, the overall process performance and transcriptome profile resulted from the $\mathrm{ambr}^{\circledR} 15$ system was representative for the $10 \mathrm{~L}$ bioreactor system. Similarly, Jayapal et al. ${ }^{30}$ used transcriptome as a fingerprint to assess the scalability of BHK cells in perfusion cultures and showed that the gene expression was similar between laboratory-scale and manufacturing-scale bioreactors. It was concluded in that study that gene expression data can substantially strengthen the confidence in scale-down model qualification compared to the traditional approach which primarily relies on cell density and product quality information. Transcriptome function analysis can also be beneficial for process development and characterization by providing a mechanistic insight into the influence of culture parameters and variations on the process performance, for example, culture temperature ${ }^{19,27,249,250}, \mathrm{DO}^{251}$, osmolality ${ }^{252}$, specific medium components ${ }^{125,249,253}$, etc. In Chapter 5, transcriptome functional analysis was used to identify genes that are differentially expressed between scales, which gives insight into differences in environmental conditions that exist between two systems of different scales and how 
they affect cell physiology. For a few genes, possible functional relationships with differences in $\mathrm{DO}$ and $\mathrm{pH}$ patterns, lactate metabolism, and glycosylation pattern were identified. The expression pattern of these genes can be used to interrogate future scale-up/down processes.

At this moment, high cost and time-demanding tasks such as complete transcriptome data processing and interpretation are still the problems for such an analysis to be carried out on a large number of samples. Studies are being undertaken to reduce the costs and to identify the genes that are associated with process performance and product quality attributes $27,249,254,255$. More rapid gene sequencing, assembly, annotation, and data processing have been achieved, and high throughput transcriptome analysis with commercial microarray systems (e.g. Affymetrix as used in Chapter 4 and 5) have become available. With further development, omics analysis or at least a subset of it can be implemented as routine analysis for high throughput culture process characterization.

\subsection{Conclusions and outlook}

During the past decades, considerable experience has been obtained on scale-up of animal cell culture processes and on development of representative scale-down bioreactor systems for these processes. Currently, miniature (mL-scale) bioreactors have been routinely incorporated into process development primarily for process parameter and clone screening studies. With the increasing pressure on shortening the development timeline and on reducing the manufacturing cost, it would be ideal if the miniature bioreactor system can largely or even completely replace the benchscale bioreactors in exploring the design space for the production-scale bioreactors. In order to do so, more results from industrial and academic users are needed to interrogate the miniature system for different process conditions and for various cell types.

As discussed in this chapter, the overall chemical environment, for example, DO and $\mathrm{pH}$ in the $\mathrm{mL}$-scale bioreactors can be kept comparable to bench-scale bioreactors. As a result, comparable process performance is normally obtained. However, although aeration in mL-scale bioreactors is sufficient to maintain similar DO as in bench-scale bioreactors, the gassing situation between the two scales is very different, which affects $\mathrm{pCO}_{2}$ and shear conditions. To improve the mL-scale bioreactors, microsparging and $\mathrm{CO}_{2}$-enriched headspace aeration may be used to deliver similar $\mathrm{pCO}_{2}$ and shear conditions as in large-scale bioreactors. In addition, better understanding of the effect of bubble size on cell death is needed. In this way, the cell death event in scale-up/down can be accounted for using a modeling approach. In terms of mixing, a similar agitation approach as large-scale bioreactors is incorporated into mL-scale bioreactors. However, the mixing time is significantly shorter in the miniature bioreactors. This will become a problem if gradients in the large scale occur that affect the cell behavior. Certainly for the intensified processes at high cell 
concentrations this may be the case. With the development of culture processes, a better understanding of the effect of gradients is needed in the near future.

Finally, the small culture volume continues to challenge the miniature systems in terms of the representative sampling volume and associated analytics and liquid handling. A high throughput cell culture system also requires high throughput sample preparation, transfer, analysis, and interpretation approaches. A higher degree of automation, for example, automated microfluidics devices and online sampling and analytical devices can be helpful to further increase the experiment and data throughput and dimension. 


Summary 

Mammalian cell culture has become the main platform for the production of therapeutic monoclonal antibodies (mAbs). Significant progress has been made during the past decades with regard to understanding culture processes, development of more robust culture processes, and shortening the development timelines. This has resulted in more affordable monoclonal antibodies in the market. The market demand for $\mathrm{mAb}$-based products is still increasing at a fast pace. In order to meet the market demand, efforts are being made to further increase product yield as well as to incorporate high-throughput technologies to accelerate the process development. In this context, this thesis aims to gain a better understanding of the fed-batch culture process and the process scale-up/down.

In Chapter 2, a clone and medium screening experiment is done in order to establish a culture platform for the subsequent process development and scale-up/down studies. In this chapter, two $\mathrm{CHO}$ cell clones derived from the same parental $\mathrm{CHO}$ cell line and producing the same monoclonal antibody (BC-G, a low producing clone; BC-P, a high producing clone) were tested in four basal media in all possible combinations with three feeds ( $=12$ conditions) in fed-batch cultures. The highest $\mathrm{mAb}$ product titer $(1.2 \mathrm{~g} / \mathrm{L})$ was obtained with ActiCHO basal medium and feeds for the BC-P clone. Moreover, this study showed that culture media and feeds have a strong impact on cell metabolism, mAb specific productivity, as well as cell size.

From Chapter 2, we selected a fed-batch process (the BC-P clone cultured in the ActiCHO media system) that resulted in a high biomass concentration (i.e. requires a high mass transfer) and $\mathrm{mAb}$ product titer for further process development and scale-up/down studies. The process was next characterized in detail at $10 \mathrm{~L}$ scale in Chapter 3 and 4 . The 10L bench-scale bioreactor system can be used as the validated small-scale model for the production-scale bioreactors. As described in Chapter 3, during the selected fed-batch process, in addition to the traditionally observed exponential growth and stationary phase, an additional phase is observed where the cell division comes to a halt but the cell growth continues with an increase in cell size. We carried out metabolic flux analysis in order to better understand the relationship between $\mathrm{mAb}$ production and cell size. It was found that during the cell size increase (SI) phase, the increase in mAb specific productivity correlated linearly with the increase in cell volume and cell dry weight. Furthermore, the amount of mAb production per mol of oxygen and amino acids consumed increased as cells grew larger ( 1.7 times higher for oxygen and $~ 3.4$ times higher for amino acids). Chapter 3 demonstrates that cell size is an important factor in a cell culture process. Furthermore, it implies that controlling cell size in a culture process may improve $\mathrm{mAb}$ production.

In Chapter 4, we moved a step forward to explore the molecular mechanisms involved in cell size regulation using transcriptome analysis. In this chapter, we studied the changes in gene expression occurring when cells started increasing in size in the process using the ActiCHO medium system. The changes in gene 
expression in another fed-batch process using FortiCHO medium system, during which no cell size increase occurred, was used as a "filter" to enrich for the genes that are related to cell size regulation. It was found out that various cyclin (cyc) and cyclin-dependent kinase (cdk) genes were significantly down-regulated in the ActiCHO process and a number of Cdk inhibitors (Cdkns) were significantly upregulated. These observations agree with the cell cycle arrest in both the G0/G1 and the G2/M phase. The mTOR upstream and downstream pathways are regulated in a strongly synchronized pattern to stimulate the mTOR activity in the ActiCHO process. This agrees with a continued cell growth after cell cycle arrest. More importantly, the activities of the cell cycle and mTOR regulator genes could be linked to the extracellular nutrient conditions through a number of cascade pathways. This suggests that by rational design of media and feeds, $\mathrm{CHO}$ cell size can be manipulated during fed-batch or perfusion processes and this can further improve cell growth and cell-specific productivity. The genes which showed a close link to cell size regulation may also be used in $\mathrm{CHO}$ cell engineering during clone development steps to further increase the productivity.

The well-characterized $10 \mathrm{~L}$ fed-batch process next served as a control for further down-scale to a $15 \mathrm{ml}$ ambr ${ }^{\circledR}$ system in Chapter 5. A miniature bioreactor system that is representative for the production scale can greatly shorten the development time and enable a more accurate definition of the process design space by increasing the data resolution and dimension. In Chapter 5, we aim to better understand the challenges in using these miniature bioreactor systems as scale-down models for the bench-scale bioreactors. The metabolism and transcriptome results obtained from the 10 L scale bioreactors in Chapter 3 and 4 are used as the process fingerprints to check the scale-down performance. The main conclusion from the comparison study was that the $\mathrm{ambr}^{\circledR} 15$ system gave in general a representative culture performance compared to the $10 \mathrm{~L}$ process in terms of cell growth, cell size, metabolism, productivity, and product quality. Two agitation scale-down approaches based on different criteria (constant agitation $\mathrm{P} / \mathrm{V}$ value and constant tip speed between scales) did not result in meaningful differences in cell physiology and overall culture performance. Transcriptome comparison showed a $6 \%$ difference in gene expression by principal component analysis (PCA) between the two scales. By looking at the differential gene expression, 2455 genes were unique to the $\mathrm{ambr}^{\circledR} 15$ systems while 1604 genes were unique to the $10 \mathrm{~L}$ system. The functional analysis of these unique genes gave a few indications on a link between differences in gene expression and differences in certain process parameters. Nevertheless, the influence of these differences on the overall comparability between the two scales was negligible. The result from Chapter 5 agrees with the literature that the ambr ${ }^{\circledR} 15$ system is in general a representative scale-down system for the bench-scale bioreactors. To this end, we are one step closer to solely using a miniature bioreactor system in exploring the process design space. In order to really achieve this, more results from industrial and 
academic users are needed to test the miniature system for different process conditions and for various cell lines.

Finally, in Chapter 6, the current knowledge and the recent advances in process scale-up/down were discussed, and improvements on the current miniature bioreactor systems are proposed. In addition, the challenges and opportunities on further implementing miniature bioreactor systems into process development were discussed. This thesis gives insight in the different biological aspects that are important for the scale-down of $\mathrm{CHO}$ fed-batch cultures, ranging from the selection and characterization of a fed-batch process to the validation of the scale-down. This will help in further shortening the development times and in developing processes that are more robust. 



\section{References}

1. Jayapal, K., Wlaschin, K., Hu, W. \& Yap, G. Recombinant protein therapeutics from CHO cells-20 years and counting. Chem. Eng. Prog. 103, 40-47 (2007).

2. Walsh, G. Biopharmaceutical benchmarks 2014. Nat. Biotechnol. 32, 992-1000 (2014).

3. Monoclonal Antibodies (mAbs) Market Size, Industry Report, 2013 - 2024. (2016).

4. Scott, A. M., Wolchok, J. D. \& Old, L. J. Antibody therapy of cancer. Nat. Rev. Cancer 12, 278-87 (2012).

5. Weiner, L. M., Surana, R. \& Wang, S. Monoclonal antibodies: versatile platforms for cancer immunotherapy. Nat. Rev. Immunol. 10, 317-327 (2010).

6. Kyriakopoulos, S. \& Kontoravdi, C. A framework for the systematic design of fed-batch strategies in mammalian cell culture. Biotechnol. Bioeng. 111, 24662476 (2014).

7. Landauer, K. Designing Media for Animal Cell Culture: CHO Cells, the Industrial Standard. Anim. Cell Biotechnol. Methods Protoc. Methods Mol. Biol. 1104, 89-103 (2014).

8. Pan, X., Streefland, M., Dalm, C., Wijffels, R. H. \& Martens, D. E. Selection of chemically defined media for $\mathrm{CHO}$ cell fed-batch culture processes. Cytotechnology 69, 39-56 (2017).

9. Lavric, V., Ofiteru, I. D. \& Woinaroschy, A. A sensitivity analysis of the fedbatch animal-cell bioreactor with respect to some control parameters. Biotechnol. Appl. Biochem. 41, 29-35 (2005).

10. Hossler, P., Khattak, S. F. \& Li, Z. J. Optimal and consistent protein glycosylation in mammalian cell culture. Glycobiology 19, 936-949 (2009).

11. Chalmers, J. J. Cells and bubbles in sparged bioreactors. Cytotechnology 15, 311320 (1994).

12. Borys, M. C., Linzer, D. I. H. \& Papoutsakis, E. T. Culture pH Affects Expression Rates and Glycosylation of Recombinant Mouse Placental Lactogen Proteins by Chinese Hamster Ovary (CHO) Cells. Nature 11, 720-724 (1993).

13. Wurm, F. M. Production of recombinant protein therapeutics in cultivated mammalian cells. Nat. Biotechnol. 22, 1393-1398 (2004).

14. Beck, A., Wurch, T., Bailly, C. \& Corvaia, N. Strategies and challenges for the next generation of therapeutic antibodies. Nat. Rev. Immunol. 10, 345-352 (2010).

15. Li, J., Wong, C. L., Vijayasankaran, N., Hudson, T. \& Amanullah, A. Feeding lactate for $\mathrm{CHO}$ cell culture processes: Impact on culture metabolism and performance. Biotechnol. Bioeng. 109, 1173-1186 (2012).

16. Xing, Z. et al. Optimizing amino acid composition of $\mathrm{CHO}$ cell culture media 
for a fusion protein production. Process Biochem. 46, 1423-1429 (2011).

17. Lu, F. et al. Automated dynamic fed-batch process and media optimization for high productivity cell culture process development. Biotechnol. Bioeng. 110, 191-205 (2013).

18. Jain, E. \& Kumar, A. Upstream processes in antibody production: Evaluation of critical parameters. Biotechnol. Adv. 26, 46-72 (2008).

19. Becerra, S., Berrios, J., Osses, N. \& Altamirano, C. Exploring the effect of mild hypothermia on CHO cell productivity. Biochem. Eng. J. 60, 1-8 (2012).

20. Kimura, R. \& Miller, W. M. Effects of elevated pCO2 and/or osmolality on the growth and recombinant tPA production of $\mathrm{CHO}$ cells. Biotechnol. Bioeng. 52, 152-160 (1996).

21. Wu Jianyong \& Goosen, M. F. Evaluation of the killing volume of gas bubbles in sparged animal cell culture bioreactors. Enzyme Microb. Technol. 17, 10361042 (1995).

22. Van't Riet, K. \& Tramper, J. Basic bioreactor design. (CRC Press, 1991).

23. Warnock, J. N. \& Al-Rubeai, M. Bioreactor systems for the production of biopharmaceuticals from animal cells. Biotechnol. Appl. Biochem. 45, 1 (2006).

24. Butler, M. \& Meneses-Acosta, a. Recent advances in technology supporting biopharmaceutical production from mammalian cells. Appl. Microbiol. Biotechnol. 96, 885-894 (2012).

25. Martens, D. E. in Cell Engineering Volume 5: Systems Biology 275-299 (2007). doi:10.1007/1-4020-5252-9

26. $\mathrm{Xu}, \mathrm{X}$. et al. The genomic sequence of the Chinese hamster ovary (CHO)-K1 cell line. Nat. Biotechnol. 29, 735-741 (2011).

27. Yee, J. C., Gerdtzen, Z. P. \& Hu, W. S. Comparative transcriptome analysis to unveil genes affecting recombinant protein productivity in mammalian cells. Biotechnol. Bioeng. 102, 246-263 (2009).

28. Khoo, S. H. G., Falciani, F. \& Al-Rubeai, M. A genome-wide transcriptional analysis of producer and non-producer NS0 myeloma cell lines. Biotechnol. Appl. Biochem. 47, 85-95 (2007).

29. Seth, G. et al. Molecular portrait of high productivity in recombinant NS0 cells. Biotechnol. Bioeng. 97, 933-951 (2007).

30. Jayapal, K. P. \& Goudar, C. T. Transcriptomics as a tool for assessing the scalability of mammalian cell perfusion systems. Mamm. Cell Cult. Biol. Manuf. 227-243 (2013).

31. Fomina-Yadlin, D., Du, Z. \& McGrew, J. T. Gene expression measurements normalized to cell number reveal large scale differences due to cell size changes, transcriptional amplification and transcriptional repression in $\mathrm{CHO}$ cells. J. Biotechnol. 189, 58-69 (2014).

32. Sieck, J. B. et al. Adaptation for survival: Phenotype and transcriptome response of $\mathrm{CHO}$ cells to elevated stress induced by agitation and sparging. J. Biotechnol. 189, 94-103 (2014). 
33. Bareither, R., Bargh, N., Oakeshott, R., Watts, K. \& Pollard, D. Automated disposable small scale reactor for high throughput bioprocess development: a proof of concept study. Biotechnol. Bioeng. 110, 3126-38 (2013).

34. Alves, C. et al. Integration of cell line and process development to overcome the challenge of a difficult to express protein. Biotechnol. Prog. 1-11 (2015).

35. Moraes, A. M., Mendonca, R. Z. \& Suazo, C. A. T. Culture media for animal cells. Anim. Cell Technol. From Biopharm. to Gene Ther. 111-128 (2008).

36. Rathore, A. S. \& Winkle, H. Quality by design for biopharmaceuticals. 27, (2009).

37. Li, F. et al. A systematic approach for scale-down model development and characterization of commercial cell culture processes. Biotechnol. Prog. 22, 696703 (2006).

38. Chu, L. \& Robinson, D. K. Industrial choices for protein production by largescale cell culture. Curr. Opin. Biotechnol. 12, 180-187 (2001).

39. Shukla, A. A. \& Thömmes, J. Recent advances in large-scale production of monoclonal antibodies and related proteins. Trends Biotechnol. 28, 253-261 (2010).

40. Nienow, A. W. Reactor Engineering in Large Scale Animal Cell Culture. Cytotechnology 50, 9-33 (2006).

41. Yang, J. et al. Fed-batch bioreactor process scale-up from 3-L to 2,500-L scale for monoclonal antibody production from cell culture. Biotechnol. Bioeng. 98, 141154 (2007).

42. Zhu, M. M. et al. Effects of Elevated pCO2 and Osmolality on Growth of CHO Cells and Production of Antibody-Fusion Protein B1: A Case Study. Biotechnol. Prog. 21, 70-77 (2005).

43. Xing, Z., Kenty, B. M., Li, Z. J. \& Lee, S. S. Scale-up analysis for a CHO cell culture process in large-scale bioreactors. Biotechnol. Bioeng. 103, 733-746 (2009).

44. Rodrigues, M. E., Costa, A. R., Henriques, M., Azeredo, J. \& Oliveira, R. Comparison of commercial serum-free media for $\mathrm{CHO}-\mathrm{K} 1$ cell growth and monoclonal antibody production. Int. J. Pharm. 437, 303-305 (2012).

45. Zagari, F. et al. High expression of the aspartate-glutamate carrier Aralar1 favors lactate consumption in $\mathrm{CHO}$ cell culture. Pharm. Bioprocess. 1, 19-27 (2013).

46. Costa, A. R. et al. The impact of cell adaptation to serum- free conditions on the glycosylation profile of a monoclonal antibody produced by Chinese hamster ovary cells. Springerplus 2, 25 (2013).

47. Reinhart, D., Damjanovic, L., Kaisermayer, C. \& Kunert, R. Benchmarking of commercially available $\mathrm{CHO}$ cell culture media for antibody production. Appl. Microbiol. Biotechnol. 99, 4645-4657 (2015).

48. Luo, J. et al. Comparative metabolite analysis to understand lactate metabolism shift in Chinese hamster ovary cell culture process. Biotechnol. Bioeng. 109, 146156 (2012). 
49. Ma, N. et al. A single nutrient feed supports both chemically defined NSO and $\mathrm{CHO}$ fed-batch processes: Improved productivity and lactate metabolism. Biotechnol. Prog. 25, 1353-1363 (2009).

50. Zamorano, F., Wouwer, A. Vande \& Bastin, G. Metabolic flux analysis of an underdetermined network of $\mathrm{CHO}$ cells. Biotechnol. Agron. Soc. Environ. 14, 603-604 (2010).

51. Mulukutla, B. C., Gramer, M. \& Hu, W. S. On metabolic shift to lactate consumption in fed-batch culture of mammalian cells. Metab. Eng. 14, 138-149 (2012).

52. Bi, J. X., Shuttleworth, J. \& Al-Rubeai, M. Uncoupling of Cell Growth and Proliferation Results in Enhancement of Productivity in p21CIP1-Arrested CHO Cells. Biotechnol. Bioeng. 85, 741-749 (2004).

53. Carvalhal, A. V., Marcelino, I. \& Carrondo, M. J. T. Metabolic changes during cell growth inhibition by p27 overexpression. Appl. Microbiol. Biotechnol. 63, 164-173 (2003).

54. Oh, S. K., Chua, F. K. \& Choo, a B. Intracellular responses of productive hybridomas subjected to high osmotic pressure. Biotechnol. Bioeng. 46, 525-35 (1995).

55. Hoffmann, E. K., Lambert, I. H. \& Pedersen, S. F. Physiology of cell volume regulation in vertebrates. Physiol. Rev. 89, 193-277 (2009).

56. Lloyd, D. R., Holmes, P., Jackson, L. P., Emery, A. N. \& Al-Rubeai, M. Relationship between cell size, cell cycle and specific recombinant protein productivity. Cytotechnology 34, 59-70 (2000).

57. Martínez, V. S., Buchsteiner, M., Gray, P., Nielsen, L. K. \& Quek, L.-E. Dynamic metabolic flux analysis using B-splines to study the effects of temperature shift on CHO cell metabolism. Metab. Eng. Commun. 2, 46-57 (2015).

58. Kim, T. K., Chung, J. Y., Sung, Y. H. \& Lee, G. M. Relationship between Cell Size and Specific Thrombopoietin Productivity in Chinese Hamster Ovary Cells during Dihydrofolate Reductase-mediated Gene Amplification. Biotechnol. Bioprocess Eng. 6, 332-336 (2001).

59. Renner, W. A., Jordan, M., Eppenberger, H. M. \& Leist, C. Cell-cell adhesion and aggregation: Influence on the growth behavior of $\mathrm{CHO}$ cells. Biotechnol. Bioeng. 41, 188-193 (1993).

60. Ahn, W. S. \& Antoniewicz, M. R. Metabolic flux analysis of CHO cells at growth and non-growth phases using isotopic tracers and mass spectrometry. Metab. Eng. 13, 598-609 (2011).

61. Martínez, V. S. et al. Flux balance analysis of $\mathrm{CHO}$ cells before and after a metabolic switch from lactate production to consumption. Biotechnol. Bioeng. 110, 660-666 (2013).

62. Young, J. D. Metabolic flux rewiring in mammalian cell cultures. Curr. Opin. Biotechnol. 24, 1108-1115 (2013).

63. Yang, M. \& Butler, M. Effects of ammonia on $\mathrm{CHO}$ cell growth, erythropoietin 
production, and glycosylation. Biotechnol Bioeng 68, 370-380 (2000).

64. Hansen, H. A. \& Emborg, C. Influence of ammonium on growth, metabolism, and productivity of a continuous suspension Chinese hamster ovary cell culture. Biotechnol. Prog. 10, 121-124 (1994).

65. Lao, M. S. \& Toth, D. Effects of ammonium and lactate on growth and metabolism of a recombinant Chinese hamster ovary cell culture. Biotechnol. Prog. 13, 688-91 (1997).

66. Fomina-Yadlin, D. et al. Cellular responses to individual amino-acid depletion in antibody-expressing and parental $\mathrm{CHO}$ cell lines. Biotechnol. Bioeng. 111, 965-79 (2014).

67. Yu, M. et al. Understanding the intracellular effect of enhanced nutrient feeding toward high titer antibody production process. Biotechnol. Bioeng. 108, 10781088 (2011).

68. Read, E. K. et al. Fermentanomics informed amino acid supplementation of an antibody producing mammalian cell culture. Biotechnol. Prog. 29, 745-53 (2013).

69. Chen, P. \& Harcum, S. W. Effects of amino acid additions on ammonium stressed CHO cells. J. Biotechnol. 117, 277-286 (2005).

70. Kishishita, S. et al. Optimization of chemically defined feed media for monoclonal antibody production in Chinese hamster ovary cells. J. Biosci. Bioeng. 120, 78-84 (2015).

71. Carinhas, N. et al. Metabolic signatures of GS-CHO cell clones associated with butyrate treatment and culture phase transition. Biotechnol. Bioeng. 110, 32443257 (2013).

72. Sengupta, N., Rose, S. T. \& Morgan, J. A. Metabolic flux analysis of CHO cell metabolism in the late non-growth phase. Biotechnol. Bioeng. 108, 82-92 (2011).

73. Templeton, N., Dean, J., Reddy, P. \& Young, J. D. Peak antibody production is associated with increased oxidative metabolism in an industrially relevant fedbatch CHO cell culture. Biotechnol. Bioeng. 110, 2013-2024 (2013).

74. Wahrheit, J., Niklas, J. \& Heinzle, E. Metabolic control at the cytosolmitochondria interface in different growth phases of CHO cells. Metab. Eng. 23, 9-21 (2014).

75. Ahn, W. S. \& Antoniewicz, M. R. Parallel labeling experiments with [1,213C]glucose and [U-13C]glutamine provide new insights into $\mathrm{CHO}$ cell metabolism. Metab. Eng. 15, 34-47 (2013).

76. Breuer, G. et al. Analysis of Fatty Acid Content and Composition in Microalgae. J. Vis. Exp. 80, e50628 (2013).

77. Cooper, M. S., Hardin, W. R., Petersen, T. W. \& Cattolico, R. A. Visualizing 'green oil' in live algal cells. J. Biosci. Bioeng. 109, 198-201 (2010).

78. DuBois, M., Gilles, K. a., Hamilton, J. K., Rebers, P. a. \& Smith, F. Colorimetric method for determination of sugars and related substances. Anal. Chem. 28, 350-356 (1956).

79. Martens, D. . \& Tramper, J. Flux Analysis of Mammalian Cell Culture. Encycl. 
Ind. Biotechnol. Encycl. Ind. Biotechnol. Bioprocess, Biosep. Cell Technol. Ed. by Michael C. Flickinger 1, 2491-2520 (2010).

80. Hefzi, H. et al. A Consensus Genome-scale Reconstruction of Chinese Hamster Ovary Cell Metabolism. Cell Syst. 3, 434-443.e8 (2016).

81. Schellenberger, J. et al. Quantitative prediction of cellular metabolism with constraint-based models: the COBRA Toolbox. Nat. Protoc. 2, 727-38 (2007).

82. Smallbone, K. \& Simeonidis, E. Flux balance analysis: A geometric perspective. J. Theor. Biol. 258, 311-315 (2009).

83. Xie, L. \& Wang, D. I. C. Applications of improved stoichiometric model in medium design and fed-batch cultivation of animal cells in bioreactor. Cytotechnology 15, 17-29 (1994).

84. Bonarius, H. P. et al. Metabolic flux analysis of hybridoma cells in different culture media using mass balances. Biotechnol. Bioeng. 50, 299-318 (1996).

85. Niklas, J., Schräder, E., Sandig, V., Noll, T. \& Heinzle, E. Quantitative characterization of metabolism and metabolic shifts during growth of the new human cell line AGE1.HN using time resolved metabolic flux analysis. Bioprocess Biosyst. Eng. 34, 533-545 (2011).

86. Altamirano, C. et al. Analysis of $\mathrm{CHO}$ cells metabolic redistribution in a glutamate-based defined medium in continuous culture. Biotechnol. Prog. 17, 1032-1041 (2001).

87. Selvarasu, S. et al. Combined in silico modeling and metabolomics analysis to characterize fed-batch CHO cell culture. Biotechnol. Bioeng. 109, 1415-1429 (2012).

88. Berlin, E., Hannah, J. S., Yamane, K., Peters, R. C. \& Howard, B. V. Fatty acid modification of membrane fluidity in Chinese hamster ovary (TR715-19) cells. Int. J. Biochem. Cell Biol. 28, 1131-9 (1996).

89. Edros, R., McDonnell, S. \& Al-Rubeai, M. The relationship between mTOR signalling pathway and recombinant antibody productivity in $\mathrm{CHO}$ cell lines. BMC Biotechnol. 14, 15 (2014).

90. Khoo, S. H. G. \& Al-Rubeai, M. Detailed understanding of enhanced specific antibody productivity in NS0 myeloma cells. Biotechnol. Bioeng. 102, 188-199 (2009).

91. Bi, J. X., Shuttleworth, J. \& Al-Rubeai, M. Uncoupling of Cell Growth and Proliferation Results in Enhancement of Productivity in p21CIP1-Arrested CHO Cells. Biotechnol. Bioeng. 85, 741-749 (2004).

92. Sun, Z., Zhou, R., Liang, S., McNeeley, K. M. \& Sharfstein, S. T. Hyperosmotic Stress in Murine Hybridoma Cells: Effects on Antibody Transcription, Translation, Posttranslational Processing, and the Cell Cycle - Sun - 2008 Biotechnology Progress - Wiley Online Library. Biotechnol. Prog. 20, 576-89 (2004).

93. Kiehl, T. R., Shen, D., Khattak, S. F., Jian Li, Z. \& Sharfstein, S. T. Observations of cell size dynamics under osmotic stress. Cytom. Part A 79 A, 560-569 (2011). 
94. Tait, A. S., Tarrant, R. D. R., Velez-Suberbie, M. L., Spencer, D. I. R. \& Bracewell, D. G. Differential response in downstream processing of $\mathrm{CHO}$ cells grown under mild hypothermic conditions. Biotechnol. Prog. 29, 688-96 (2013).

95. Bernson, V. S. M. Acetyl-CoA Hydrolase; Activity, Regulation and Physiological, Significance of the Enzyme in Brown Adipose Tissue from Hamster. Eur. J. Biochem. 67, 403-410 (1976).

96. Tanaka, K., Budd, M. A., Efron, M. L. \& Isselbacher, K. J. Isovaleric acidemia: a new genetic defect of leucine metabolism. Proc. Natl. Acad. Sci. 56, 236-242 (1966).

97. Ihrig, T. J., Maulawizada, M. A., Thomas, B. D. \& Jacobson, F. S. in Animal Cell technology: developments towards the 21st century 193-197 (Springer, 1995).

98. Duarte, T. M. et al. Metabolic responses of $\mathrm{CHO}$ cells to limitation of key amino acids. Biotechnol. Bioeng. 111, 2095-2106 (2014).

99. Liu, P. et al. Chinese Hamster Ovary K2 Cell Lipid Droplets Appear to be Metabolic Organelles Involved in Membrane Traffic. J. Biol. Chem. 279, 37873792 (2004).

100. Zagari, F., Jordan, M., Stettler, M., Broly, H. \& Wurm, F. M. Lactate metabolism shift in $\mathrm{CHO}$ cell culture: The role of mitochondrial oxidative activity. $N$. Biotechnol. 30, 238-245 (2013).

101. Martinelle, K. \& Häggström, L. Mechanisms of ammonia and ammonium ion toxicity in animal cells: Transport across cell membranes. J. Biotechnol. 30, 339350 (1993).

102. Terada, H. Uncouplers of oxidative phosphorylation. Environ. Health Perspect. 87, 213-218 (1990).

103. Pressman, B. C. \& Lardy, H. A. Effect of surface active agents on the latent ATPASE of mitochondira. Biochim. Biophys. Acta 21, 458-466 (1956).

104. Skulachev, V. P. Fatty acid circuit as a physiological mechanism of uncoupling of oxidative phosphorylation. FEBS Lett. 294, 158-162 (1991).

105. Rohde, J., Heitman, J. \& Cardenas, M. E. The TOR Kinases Link Nutrient Sensing to Cell Growth. J. Biol. Chem. 276, 9583-9586 (2001).

106. Fingar, D. C., Salama, S., Tsou, C., Harlow, E. \& Blenis, J. Mammalian cell size is controlled by mTOR and its downstream targets S6K1 and 4EBP1/eIF4E. Genes Dev. 16, 1472-1487 (2002).

107. Conlon, I. \& Raff, M. Size control in animal development. Cell 96, 235-244 (1999).

108. Jorgensen, P. \& Tyers, M. How cells coordinate growth and division. Curr. Biol. 14, 1014-1027 (2004).

109. Manning, J. J. H. and B. D. mTOR couples cellular nutrient sensing to organismal metabolic homeostasis. 100, 130-134 (2012).

110. Laplante, M. \& Sabatini, D. M. mTOR signaling at a glance. J Cell Sci 122, 35893594 (2009).

111. Dennis, P. B. et al. Mammalian TOR: a homeostatic ATP sensor. Science 294, 
1102-1105 (2001).

112. Dreesen, I. A. J. \& Fussenegger, M. Ectopic expression of human mTOR increases viability, robustness, cell size, proliferation, and antibody production of chinese hamster ovary cells. Biotechnol. Bioeng. 108, 853-866 (2011).

113. Backman, S. A. et al. Deletion of Pten in mouse brain causes seizures, ataxia and defects in soma size resembling Lhermitte-Duclos disease. Nat. Genet. 29, 396-403 (2001).

114. McVey, D. et al. CHO cells knocked out for TSC2 display an improved productivity of antibodies under fed batch conditions. Biotechnol. Bioeng. 113, 1942-1952 (2016).

115. Vermeulen, K. et al. The cell cycle:a review of regulation,deregulation and therapeutic targets in cancer. Cell Prolif. 36, 131-149 (2003).

116. Pan, X., Dalm, C., Wijffels, R. H. \& Martens, D. E. Metabolic characterization of a CHO cell size increase phase in fed-batch cultures. Appl. Microbiol. Biotechnol. 101, 8101-8113 (2017).

117. Lin, K. et al. MADMAX - Management and analysis database for multiple omics experiments. J. Integr. Bioinform. 8, 160 (2011).

118. Irizarry, R. A. et al. Exploration, normalization, and summaries of high density oligonucleotide array probe level data. Biostatistics 4, 249-264 (2003).

119. Dai, M. et al. Evolving gene/transcript definitions significantly alter the interpretation of GeneChip data. Nucleic Acids Res. 33, 1-9 (2005).

120. Ritchie, M. E. et al. limma powers differential expression analyses for RNAsequencing and microarray studies. Nucleic Acids Res. (2015).

121. Sartor, M. A. et al. Intensity-based hierarchical Bayes method improves testing for differentially expressed genes in microarray experiments. BMC Bioinformatics 7, 538 (2006).

122. Benjamini, Y. \& Hochberg, Y. Controlling the false discovery rate: a practical and powerful approach to multiple testing. J. R. Stat. Soc. Ser. B 289-300 (1995).

123. Allison, D. B., Cui, X., Page, G. P. \& Sabripour, M. Microarray data analysis: from disarray to consolidation and consensus. Nat. Rev. Genet. 7, 55-65 (2006).

124. Yuk, I. H. et al. Effects of copper on CHO cells: Cellular requirements and product quality considerations. Biotechnol. Prog. 226-238 (2014). doi:10.1002/btpr.2004

125. Yuk, I. H. et al. Effects of copper on cho cells: Insights from gene expression analyses. Biotechnol. Prog. 1-34 (2014).

126. Cooper, G. M. \& Hausman, R. E. The cell. (Sinauer Associates Sunderland, 2000).

127. Marchant, R. J., Al-Fageeh, M. B., Underhill, M. F., Racher, A. J. \& Smales, C. M. Metabolic Rates, Growth Phase, and mRNA Levels Influence Cell-Specific Antibody Production Levels from In Vitro-Cultured Mammalian Cells at SubPhysiological Temperatures. Mol. Biotechnol. 39, 69-77 (2008).

128. Kaufmann, H., Mazur, X., Fussenegger, M. \& Bailey, J. E. Influence of low temperature on productivity, proteome and protein phosphorylation of $\mathrm{CHO}$ 
cells. Biotechnol. Bioeng. 63, 573-582 (1999).

129. Kim, N. S. \& Lee, G. M. Response of recombinant Chinese hamster ovary cells to hyperosmotic pressure: effect of Bcl-2 overexpression. J. Biotechnol. 95, 237248 (2002).

130. Du, Z. et al. Use of a small molecule cell cycle inhibitor to control cell growth and improve specific productivity and product quality of recombinant proteins in CHO cell cultures. Biotechnol. Bioeng. 112, 141-155 (2015).

131. Xu, Y. et al. PAMM: a redox regulatory protein that modulates osteoclast differentiation. Antioxid. Redox Signal. 13, 27-37 (2010).

132. Chen et al. Nuclear Export of Smads by RanBP3L Regulates Bone Morphogenetic Protein Signaling and Mesenchymal Stem Cell Differentiation. Mol. Cell. Biol. 35, 1700-1711 (2015).

133. Hemmings, B. A. \& Restuccia, D. F. PI3K-PKB / Akt Pathway. 1-4 (2012).

134. Berwick, D. C., Hers, I., Heesom, K. J., Kelly Moule, S. \& Tavaré, J. M. The identification of ATP-citrate lyase as a protein kinase B (Akt) substrate in primary adipocytes. J. Biol. Chem. 277, 33895-33900 (2002).

135. Ma, X. M. \& Blenis, J. Molecular mechanisms of mTOR-mediated translational control. Nat.Rev.Mol.Cell Biol. 5, 827-835 (2004).

136. Mulukutla, B. C., Yongky, A., Le, T., Mashek, D. G. \& Hu, W. S. Regulation of Glucose Metabolism - A Perspective From Cell Bioprocessing. Trends Biotechnol. 34, 638-651 (2016).

137. Satyanarayana, A. \& Kaldis, P. Mammalian cell-cycle regulation: several Cdks, numerous cyclins and diverse compensatory mechanisms. Oncogene 28, 292539 (2009).

138. Girard, F., Strausfeld, U., Fernandez, A. \& Lamb, N. J. C. Cyclin a is required for the onset of DNA replication in mammalian fibroblasts. Cell 67, 1169-1179 (1991).

139. Ohtsubo, M., Theodoras, a M., Schumacher, J., Roberts, J. M. \& Pagano, M. Human cyclin E, a nuclear protein essential for the G1-to-S phase transition. Mol. Cell. Biol. 15, 2612-24 (1995).

140. Coqueret, $\mathrm{O}$. New roles for p21 and p27 cell-cycle inhibitors: A function for each cell compartment? Trends Cell Biol. 13, 65-70 (2003).

141. Shieh, S. Y., Ahn, J., Tamai, K., Taya, Y. \& Prives, C. The human homologs of checkpoint kinases Chk1 and Cds1 (Chk2) phosphorylate, p53 at multiple DNA damage-inducible sites. Genes Dev. 14, 289-300 (2000).

142. Massague, J. \& Lo, R. S. TGF- $\beta$ Signaling in Growth Control, Cancer, and Heritable Disorders. Cell 103, 295-309 (2000).

143. Datto, M. B. et al. Transforming growth factor beta induces the cyclindependent kinase inhibitor p21 through a p53-independent mechanism. Proc. Natl. Acad. Sci. U. S. A. 92, 5545-9 (1995).

144. Massagué, J. TGF $\beta$ signalling in context. Nat. Rev. Mol. Cell Biol. 13, 616-30 (2012). 
145. Barcellos-Hoff, M. H. \& Dix, T. A. Redox-mediated activation of latent transforming growth factor-beta 1. Mol. Endocrinol. 10, 1077-1083 (1996).

146. Lyons, R. M., Keski-Oja, J. \& Moses, H. L. Proteolytic activation of latent transforming growth factor-beta from fibroblast-conditioned medium. J. Cell Biol. 106, 1659-1665 (1988).

147. Yu, Q. \& Stamenkovic, I. Cell surface-localized matrix metalloproteinase-9 proteolytically activates TGF- $\beta$ and promotes tumor invasion and angiogenesis. Genes Dev. 14, 163-176 (2000).

148. Jong Kyong, K. I. M. \& Alan Diehl, J. Nuclear cyclin D1: An oncogenic driver in human cancer. J. Cell. Physiol. 220, 292-296 (2009).

149. Schmelzle, T. \& Hall, M. N. TOR, a central controller of cell growth. Cell 103, 253-262 (2000).

150. Richter, J. D. \& Sonenberg, N. Regulation of cap-dependent translation by eIF4E inhibitory proteins. Nature 433, 477-480 (2005).

151. Lamming, D. W. \& Sabatini, D. M. A central role for mTOR in lipid homeostasis. Cell Metab. 18, 465-469 (2013).

152. Porstmann, T. et al. SREBP Activity Is Regulated by mTORC1 and Contributes to Akt-Dependent Cell Growth. Cell Metab. 8, 224-236 (2008).

153. Lum, J. J. et al. Growth factor regulation of autophagy and cell survival in the absence of apoptosis. Cell 120, 237-248 (2005).

154. Hwa, V., Oh, Y. \& Rosenfeld, R. G. Insulin-like growth factor binding proteins: a proposed superfamily. Acta Pædiatrica 88, 37-45 (1999).

155. Franke, T. F., Hornik, C. P., Segev, L., Shostak, G. A. \& Sugimoto, C. PI3K/Akt and apoptosis: size matters. Oncogene 22, 8983-98 (2003).

156. Jewell, J. L., Russell, R. C. \& Guan, K. Amino acid signalling upstream. Nat. Rev. Mol. Cell Biol. 14, 133-139 (2013).

157. Mulukutla, B. C., Khan, S., Lange, A. \& Hu, W.-S. Glucose metabolism in mammalian cell culture: new insights for tweaking vintage pathways. Trends Biotechnol. 28 VN-r, 476-484 (2010).

158. Brancolini, C., Benedetti, M. \& Schneider, C. Microfilament reorganization during apoptosis: the role of Gas2, a possible substrate for ICE-like proteases. EMBO J. 14, 5179-90 (1995).

159. Nagai, K. et al. Gas6 induces Akt/mTOR-mediated mesangial hypertrophy in diabetic nephropathy. Kidney Int. 68, 552-561 (2005).

160. Del Sal, G., Ruaro, M. E., Philipson, L. \& Schneider, C. The growth arrestspecific gene, gas1, is involved in growth suppression. Cell 70, 595-607 (1992).

161. She, B.-R., Liou, G.-G. \& Lin-Chao, S. Association of the growth-arrest-specific protein Gas7 with F-actin induces reorganization of microfilaments and promotes membrane outgrowth. Exp. Cell Res. 273, 34-44 (2002).

162. Smith, C. M. \& Steitz, J. A. Classification of gas5 as a multi-small-nucleolarRNA (snoRNA) host gene and a member of the 5'-terminal oligopyrimidine gene family reveals common features of snoRNA host genes. Mol. Cell. Biol. 18, 
6897-909 (1998).

163. Ma, C. et al. The growth arrest-specific transcript 5 (GAS5): a pivotal tumor suppressor long noncoding RNA in human cancers. Tumor Biol. 37, 1437-1444 (2016).

164. Petroulakis, E. et al. p53-Dependent Translational Control of Senescence and Transformation via 4E-BPs. Cancer Cell 16, 439-446 (2009).

165. Xu, P. et al. Characterization of TAP Ambr 250 disposable bioreactors, as a reliable scale-down model for biologics process development. Biotechnol. Prog. (2016).

166. Sani, M. H. Evaluation of Microwell based Systems and Miniature Bioreactors for Rapid Cell Culture Bioprocess Development and Scale-up. 1-214 (2016).

167. Betts, J. I. \& Baganz, F. Miniature bioreactors: current practices and future opportunities. Microb. Cell Fact. 5, 21 (2006).

168. Nienow, A. W. et al. The physical characterisation of a microscale parallel bioreactor platform with an industrial $\mathrm{CHO}$ cell line expressing an IgG4. Biochem. Eng. J. 76, 25-36 (2013).

169. Hsu, W. T., Aulakh, R. P. S., Traul, D. L. \& Yuk, I. H. Advanced microscale bioreactor system: A representative scale-down model for bench-top bioreactors. Cytotechnology 64, 667-678 (2012).

170. Moses, S. \& Manahan, M. Assessment of AMBRTM as a model for highthroughput cell culture process development strategy. Adv. Biosci. .. 2012, 918927 (2012).

171. Nienow, A. W. et al. Scale-down studies for assessing the impact of different stress parameters on growth and product quality during animal cell culture. Chem. Eng. Res. Des. 91, 2265-2274 (2013).

172. Rameez, S., Mostafa, S. S., Miller, C. \& Shukla, A. A. High-throughput miniaturized bioreactors for cell culture process development: Reproducibility, scalability, and control. Biotechnol. Prog. 30, 718-727 (2014).

173. Rouiller, Y. et al. Screening and assessment of performance and molecule quality attributes of industrial cell lines across different fed-batch systems. Biotechnol. Prog. 32, 160-170 (2016).

174. Janakiraman, V., Kwiatkowski, C., Kshirsagar, R., Ryll, T. \& Huang, Y.-M. Application of high-throughput mini-bioreactor system for systematic scaledown modeling, process characterization, and control strategy development. Biotechnol. Prog. (2015).

175. Siva, S. et al. Leveraging high-throughput technology to accelerate the time to clinic: A case study of a mAb. Eng. Life Sci. 143-151 (2015).

176. Farrell, A., McLoughlin, N., Milne, J. J., Marison, I. W. \& Bones, J. Application of multi-omics techniques for bioprocess design and optimization in Chinese hamster ovary cells. J. Proteome Res. 13, 3144-3159 (2014).

177. Sieck, J. B. et al. Development of a Scale-Down Model of hydrodynamic stress to study the performance of an industrial $\mathrm{CHO}$ cell line under simulated 
production scale bioreactor conditions. J. Biotechnol. 164, 41-49 (2013).

178. Trummer, E. et al. Process parameter shifting: Part I. Effect of DOT, pH, and temperature on the performance of Epo-Fc expressing $\mathrm{CHO}$ cells cultivated in controlled batch bioreactors. Biotechnol. Bioeng. 94, 1033-1044 (2006).

179. Serrato, J. A., Palomares, L. A., Meneses-Acosta, A. \& Ramírez, O. T. Heterogeneous conditions in dissolved oxygen affect $\mathrm{N}$-glycosylation but not productivity of a monoclonal antibody in hybridoma cultures. Biotechnol. Bioeng. 88, 176-188 (2004).

180. Seagroves, T. N. et al. Transcription factor HIF-1 is a necessary mediator of the pasteur effect in mammalian cells. Mol. Cell. Biol. 21, 3436-44 (2001).

181. Poole, R. C. \& Halestrap, A. P. Transport of lactate and other monocarboxylates across mammalian plasma membranes. Am. J. Physiol. 264, C761-82 (1993).

182. Halestrap, A. P. \& Price, N. T. The proton-linked monocarboxylate transporter (MCT) family: structure, function and regulation. Biochem J 343 Pt 2, 281-299 (1999).

183. Health, C. \& Kiss, R. Cell culture process development: advances in process engineering. Biotechnol Prog. 23, 46-51 (2007).

184. Nielsen, J., Villadsen, J. \& Lidén, G. Scale-up of bioprocesses. Bioreact. Eng. Princ. 477-518 (2003).

185. Lara, A. R., Galindo, E., Ramírez, O. T. \& Palomares, L. a. Living with heterogeneities in bioreactors: understanding the effects of environmental gradients on cells. Mol. Biotechnol. 34, 355-381 (2006).

186. Kim, B. J., Diao, J. \& Shuler, M. L. Mini-scale bioprocessing systems for highly parallel animal cell cultures. Biotechnol. Prog. 28, 595-607 (2012).

187. Löffelholz, C. et al. Bioengineering parameters for single-use bioreactors: Overview and evaluation of suitable methods. Chemie-Ingenieur-Technik 85, 4056 (2013).

188. Betts, J. I., Doig, S. D. \& Baganz, F. Characterization and application of a miniature $10 \mathrm{~mL}$ stirred-tank bioreactor, showing scale-down equivalence with a conventional 7 L reactor. Biotechnol. Prog. 22, 681-688 (2006).

189. Bhambure, R., Kumar, K. \& Rathore, A. S. High-throughput process development for biopharmaceutical drug substances. Trends Biotechnol. 29, 127135 (2011).

190. Bareither, R. \& Pollard, D. A review of advanced small-scale parallel bioreactor technology for accelerated process development: current state and future need. Biotechnol. Prog. 27, 2-14 (2011).

191. Amanullah, A. et al. Novel micro-bioreactor high throughput technology for cell culture process development: Reproducibility and scalability assessment of fed-batch CHO cultures. Biotechnol. Bioeng. 106, (2010).

192. Pollard, J., Mcdonald, P. \& Hesslein, A. Lessons learned in building highthroughput process development capabilities. Eng. Life Sci. 16, 93-98 (2016).

193. Bareither, R., Bargh, N., Oakeshott, R., Watts, K. \& Pollard, D. Automated 
disposable small scale reactor for high throughput bioprocess development: A proof of concept study. Biotechnol. Bioeng. 110, 3126-3138 (2013).

194. Oosterhuis, N. M. G. Scale-down of bioreactors. (1984).

195. Papagianni, M. Methodologies for Scale-down of Microbial Bioprocesses. J. Microb. Biochem. Technol. 5, 1-7 (2011).

196. Marques, M. P. C., Cabral, J. M. S. \& Fernandes, P. Bioprocess scale-up: Quest for the parameters to be used as criterion to move from microreactors to labscale. J. Chem. Technol. Biotechnol. 85, 1184-1198 (2010).

197. Miller, W. M., Wilke, C. R. \& Blanch, H. W. Effects of dissolved oxygen concentration on hybridoma growth and metabolism in continuous culture. $J$. Cell. Physiol. 132, 524-530 (1987).

198. Garcia-Ochoa, F. \& Gomez, E. Bioreactor scale-up and oxygen transfer rate in microbial processes: An overview. Biotechnol. Adv. 27, 153-176 (2009).

199. Bonarius, H. P., de Gooijer, C. D., Tramper, J. \& Schmid, G. Determination of the respiration quotient in mammalian cell culture in bicarbonate buffered media. Biotechnol. Bioeng. 45, 524-35 (1995).

200. Schmelzer, A. E. \& Miller, W. M. Hyperosmotic stress and elevated pCO2 alter monoclonal antibody charge distribution and monosaccharide content. Biotechnol. Prog. 18, 346-353 (2002).

201. deDezengotita, V. M., Kimura, R. \& Miller, W. M. Effects of CO2 and osmolality on hybridoma cells: growth, metabolism and monoclonal antibody production. Cytotechnology 28, 213-227 (1998).

202. Mostafa, S. S. \& Gu, X. Strategies for improved dCO2 removal in large-scale fed-batch cultures. Biotechnol. Prog. 19, 45-51 (2003).

203. Gray, D. R., Chen, S., Howarth, W., Inlow, D. \& Maiorella, B. L. CO2 in largescale and high-density $\mathrm{CHO}$ cell perfusion culture. Cytotechnology 22, 65-78 (1996).

204. Sieblist, C. et al. Insights into large-scale cell-culture reactors: II. Gas-phase mixing and CO 2 stripping. Biotechnol. J. 6, 1547-1556 (2011).

205. Ozturk, S. S. Engineering challenges in high density cell culture systems. Cytotechnology 22, 3-16 (1996).

206. Sieblist, C., Jenzsch, M., Pohlscheidt, M. \& Lübbert, A. Insights into large-scale cell-culture reactors: I. Liquid mixing and oxygen supply. Biotechnol. J. 6, 15321546 (2011).

207. Varley, J. \& Birch, J. Reactor design for large scale suspension animal cell culture. Cytotechnology 29, 177-205 (1999).

208. Xing, Z., Lewis, A. M., Borys, M. C. \& Li, Z. J. A carbon dioxide stripping model for mammalian cell culture in manufacturing scale bioreactors. Biotechnol. Bioeng. 114, 1184-1194 (2016).

209. $\mathrm{Xu}, \mathrm{S}$. et al. A practical approach in bioreactor scale-up and process transfer using a combination of constant $\mathrm{P} / \mathrm{V}$ and vvm as the criterion. Biotechnol. Prog. (2017). 
210. Sweere, A. P. J., Luyben, K. C. A. M. \& Kossen, N. W. F. Regime Analysis and Scale-down: Tools to Investigate the Performance of Bioreactors. Enzyme Microb. Technol. 9, 386-398 (1987).

211. Amanullah, A., McFarlane, C. M., Emery, A. N. \& Nienow, A. W. Scale-down model to simulate spatial $\mathrm{pH}$ variations in large-scale bioreactors. Biotechnol. Bioeng. 73, 390-399 (2001).

212. Tramper, J. Oxygen gradients in animal-cell bioreactors. Cytotechnology 18, 2734 (1995).

213. Brunner, M. et al. The impact of $\mathrm{pH}$ inhomogeneities on $\mathrm{CHO}$ cell physiology and fed-batch process performance-two-compartment scale-down modelling and intracellular pH excursion. Biotechnol. J. (2017).

214. Kaiser, S. C., Löffelholz, C., Werner, S. \& Eibl, D. CFD for characterizing standard and single-use stirred cell culture bioreactors. Comput. Fluid Dyn. Technol. Appl. 97-122 (2011).

215. Godoy-Silva, R., Chalmers, J. J., Casnocha, S. A., Bass, L. A. \& Ma, N. Physiological responses of $\mathrm{CHO}$ cells to repetitive hydrodynamic stress. Biotechnol. Bioeng. 103, 1103-1117 (2009).

216. Godoy-Silva, R., Mollet, M. \& Chalmers, J. J. Evaluation of the effect of chronic hydrodynamical stresses on cultures of suspensed CHO-6E6 cells. Biotechnol. Bioeng. 102, 1119-1130 (2009).

217. Ma, N., Koelling, K. W. \& Chalmers, J. J. Fabrication and use of a transient contractional flow device to quantify the sensitivity of mammalian and insect cells to hydrodynamic forces. Biotechnol. Bioeng. 80, 428-437 (2002).

218. Legmann, R. et al. A predictive high-throughput scale-down model of monoclonal antibody production in CHO cells. Biotechnol. Bioeng. 104, 11071120 (2009).

219. Chalmers, J. J. Mixing, aeration and cell damage, 30+ years later: What we learned, how it affected the cell culture industry and what we would like to know more about. Curr. Opin. Chem. Eng. 10, 94-102 (2015).

220. Cherry, R. S. Animal cells in turbulent fluids: Details of the physical stimulus and the biological response. Biotechnol. Adv. 11, 279-299 (1993).

221. Jöbses, I., Martens, D. \& Tramper, J. Lethal events during gas sparging in animal cell culture. Biotechnol. Bioeng. 37, 484-490 (1991).

222. Trinh, K., Garcia-Briones, M., Chalmers, J. J. \& Hink, F. Quantification of damage to suspended insect cells as a result of bubble rupture. Biotechnol. Bioeng. 43, 37-45 (1994).

223. Garcia-Briones, M. a., Brodkey, R. S. \& Chalmers, J. J. Computer simulations of the rupture of a gas bubble at a gas-liquid interface and its implications in animal cell damage. Chem. Eng. Sci. 49, 2301-2320 (1994).

224. Boulton-Stone, J. M. \& Blake, J. R. Gas bubbles bursting at a free surface. J. Fluid Mech. 254, 437-466 (1993).

225. Ghebeh, H., Gillis, J. \& Butler, M. Measurement of hydrophobic interactions of 
mammalian cells grown in culture. 95, 39-48 (2002).

226. Chattopadhyay, D., Rathman, J. F. \& Chalmers, J. J. The protective effect of specific medium additives with respect to bubble rupture. Biotechnol. Bioeng. 45, 473-480 (1995).

227. Tharmalingam, T., Ghebeh, H., Wuerz, T. \& Butler, M. Pluronic Enhances the Robustness and Reduces the Cell Attachment of Mammalian Cells. Mol. Biotechnol. 39, 167-177 (2008).

228. Ma, N., Chalmers, J. J., Auniņš, J. G., Zhou, W. \& Xie, L. Quantitative Studies of Cell-Bubble Interactions and Cell Damage at Different Pluronic F-68 and Cell Concentrations. Biotechnol. Prog. 20, 1183-1191 (2004).

229. Murhammer, D. W. \& Goochee, C. F. Sparged Animal Cell Bioreactors: Mechanism of Cell Damage and Pluronic F-68 Protection. Biotechnol. Prog. 6, 391-397 (1990).

230. Barbosa, M. J., Hadiyanto \& Wijffels, R. H. Overcoming Shear Stress of Microalgae Cultures in Sparged Photobioreactors. Biotechnol. Bioeng. 85, 78-85 (2004).

231. Zhu, Y., Cuenca, J. V., Zhou, W. \& Varma, A. NS0 cell damage by high gas velocity sparging in protein-free and cholesterol-free cultures. Biotechnol. Bioeng. 101, 751-760 (2008).

232. Liu, Y., Li, F., Hu, W., Wiltberger, K. \& Ryll, T. Effects of bubble-liquid twophase turbulent hydrodynamics on cell damage in sparged bioreactor. Biotechnol. Prog. 30, 48-58 (2014).

233. Tramper, J., Williams, J. B., Joustra, D. \& Vlak, J. M. Shear sensitivity of insect cells in suspension. Enzyme Microb. Technol. 8, 33-36 (1986).

234. Tramper, J., Smit, D., Straatman, J. \& Vlak, J. M. Bubble-column design for growth of fragile insect cells. Bioprocess Eng. 3, 37-41 (1988).

235. Wlaschin, K. F. \& Hu, W. S. Fedbatch culture and dynamic nutrient feeding. Adv. Biochem. Eng. Biotechnol. 101, 43-74 (2006).

236. Schmidt, P. M. et al. A robust robotic high-throughput antibody purification platform. J. Chromatogr. A 1455, 9-19 (2016).

237. Welsh, J. P. et al. A practical strategy for using miniature chromatography columns in a standardized high-throughput workflow for purification development of monoclonal antibodies. Biotechnol. Prog. 30, 626-635 (2014).

238. Rathore, A. S. \& Bhambure, R. in Protein Downstream Processing: Design, Development and Application of High and Low-Resolution Methods (ed. Labrou, N. E.) 29-37 (Humana Press, 2014).

239. Burnina, I., Hoyt, E., Lynaugh, H., Li, H. \& Gong, B. A cost-effective platebased sample preparation for antibody N-glycan analysis. J. Chromatogr. A 1307, 201-206 (2013).

240. Doherty, M. et al. An automated robotic platform for rapid profiling oligosaccharide analysis of monoclonal antibodies directly from cell culture. Anal. Biochem. 442, 10-18 (2013). 
241. Yang, X. et al. Ultrafast and high-throughput N-glycan analysis for monoclonal antibodies. MAbs 8, 706-717 (2016).

242. Wang, Y., Feng, P., Sosic, Z. \& Zang, L. Analytical \& Bioanalytical Techniques Monitoring Glycosylation Profile and Protein Titer in Cell Culture Samples Using ZipChip CE-MS. 8, (2017).

243. Delouvroy, F. et al. ambr ${ }^{\mathrm{TM}}$ Mini-bioreactor as a high-throughput tool for culture process development to accelerate transfer to stainless steel manufacturing scale: comparability study from process performance to product quality attributes. BMC Proc. 9, P78 (2015).

244. Linshiz, G. et al. PR-PR: Cross-platform laboratory automation system. ACS Synth. Biol. 3, 515-524 (2014).

245. Linshiz, G. et al. PaR-PaR laboratory automation platform. ACS Synth. Biol. 2, 216-222 (2013).

246. Downey, B. J., Graham, L. J., Breit, J. F. \& Glutting, N. K. A novel approach for using dielectric spectroscopy to predict viable cell volume (VCV) in early process development. Biotechnol. Prog. 30, 479-487 (2014).

247. Li, B., Ray, B. H., Leister, K. J. \& Ryder, A. G. Performance monitoring of a mammalian cell based bioprocess using Raman spectroscopy. Anal. Chim. Acta 796, 84-91 (2013).

248. Sandor, M. et al. Comparative study of non-invasive monitoring via infrared spectroscopy for mammalian cell cultivations. J. Biotechnol. 168, 636-645 (2013).

249. Kantardjieff, A. et al. Transcriptome and proteome analysis of Chinese hamster ovary cells under low temperature and butyrate treatment. J. Biotechnol. 145, 143-159 (2010).

250. Gammell, P., Barron, N., Kumar, N. \& Clynes, M. Initial identification of low temperature and culture stage induction of miRNA expression in suspension CHO-K1 cells. J. Biotechnol. 130, 213-218 (2007).

251. Qian, Y. et al. Hypoxia influences protein transport and epigenetic repression of CHO cell cultures in shake flasks. Biotechnol. J. 9, 1413-1424 (2014).

252. Shen, D. et al. Transcriptomic responses to sodium chloride-induced osmotic stress: A study of industrial fed-batch $\mathrm{CHO}$ cell cultures. Biotechnol. Prog. 26, 1104-1115 (2010).

253. Qian, Y. et al. Cell culture and gene transcription effects of copper sulfate on Chinese hamster ovary cells. Biotechnol. Prog. 27, 1190-1194 (2011).

254. Nissom, P. M. et al. Transcriptome and proteome profiling to understanding the biology of high productivity CHO cells. Mol. Biotechnol. 34, 125-140 (2006).

255. Zhao, L., Fu, H.-Y., Raju, R., Vishwanathan, N. \& Hu, W.-S. Unveiling gene trait relationship by cross-platform meta-analysis on Chinese hamster ovary cell transcriptome. Biotechnol. Bioeng. 9999, 1-10 (2017). 



\section{Acknowledgement}

This Ph.D adventure is coming to an end. There are many people I want to thank for joining me alongside this journey, making it so much colorful.

First of all, I want to than my promoter René Wijffels. It has been a great honor to work in your group and to learn from your immense experience and knowledge. I want to express my deepest gratitude to my two supervisors, Dirk Martens and Ciska Dalm. Dirk, I could not have imagined having a better mentor than you for my Ph.D study, and I could not express enough thanks to your continuous help during this journey. Every discussion with you has always brought me insight and encouragement to my research. Ciska, thank you for asking so many times "What do you want to say? What is your main message?" More often than not I found that they were difficult questions before I realized that the message was not very clear even to myself. Working at Synthon with your team was an amazing experience. I wish I could work in a team like that someday. My sincere thanks also goes to my former supervisor, Mathieu Streefland, who introduced me to the cell culture world and who provided me with an opportunity to join BPE. "There is a thin layer between directness and rudeness." Your life-coaching quote is still stabbing in my mind.

I would like to thank a number of academic staffs who offered great helps to my Ph.D project. My Ph.D fellow, Abdulaziz Al Sayyari. Aziz, it was a pleasure working with you in the lab and combining your transcriptome knowledge in this thesis. They have surely widened my view and thickened the content of this research. I also want to thank Nienke Vriezen and Louis Boon, who are the "industrial partners" of this project. Nienke, many of your remarks have helped me to build a critical way of thinking. Your comments have brought a clearer logical line to Chapter 5. Louis, thank you very much for always being there to help in every single project meetings. And it was an honor to have you as one of my opponents for my defense. Michel Eppink, thank you for overviewing and tracking the whole project flow and for the encouragement towards the end of my thesis.

I owe my big thanks to my MSc. and BSc. students: Jannik, Danial, Daphne, Renna, Lisa, Ivan, Jianan, and Chengnan. You brought so many brilliant ideas to this project and you all made the lab work so vivid and enjoyable. It was my pleasure to have hosted you in the BPE group and in the cell culture world during your studies.

I want to thank Alison Young for inspiring the proposition 6. I also want to thank Walnut for inspiring the proposition 4.

I'd like to thank the people who started in BPE on the same day as me, the people who we built the BPE "golden era" together: Ilse, Joao, Richard, and Hans. Joao, my brother, I believe our names are still confusing some people now. I was proud of the vegan challenge I had with you and I think I will try it again and will brag about it 
many times in the future. Mitsue, the international group was always lively with you. I wish I could see you in Mexico someday in the future. Somehow, you and Rupali teasing Douwe in the coffee corner was just too hilarious and iconic for me. Ilse, we knew each other since our master times. To me, it was a precious memory to share the similar path that we walked through. And OMG! That was like yesterday! Rupali, how come that I still taste the Indian food you made? I guess there is only one explanation. Richard, the big father of the two, I guess only until now can I truly understand the smile on your face when talking about your angels. Tim, the topics I remembered having with you were...why were they all about women? Pauline, it could not be too wrong that a beauty like you speaking such a beautiful language. But why all I learnt was an " $\mathrm{M}$ " word that I don't even dare to type here? Jorijn, I really enjoyed working in the lab next to yours - you were always there! As I am thinking of it, I could still see your silhouette through the portlight window between our labs. To all my international dinner group folks, Mitsue, Ilse, Joao, Jorijn, Gerard, Aziz, Agi, Rupali, Ruchir, Luci, Imma, Marta, Paulo. You are such a beautiful scenery on this journey! I doubt if I ever get the chance to work and hang out in such a multinational group again. Of course, I owe my thanks to the famous and amazing technician office members: Wendy, Fred, Sebastiaan, Snezana, Bregje. You clear all the doubts for us to do the pure research. It's you who made the BPE such a lovely place to work. To my cell culture bro, Aziz, we can never stop making jokes about everything in the lab. And I have to say that the actions you made on the soccer field with your big belly is damn good! However, I did wish to see your sixpacks. Jorijn, Gerard, Tim, Richard, Stephanie, Pauline, Kiira, and Enrico, it was a pleasure for me to share office with all of you. The office jokes are always so enlightening and refreshing. I want to give thanks particularly to my paranymphs, Yifan and Ilse. You have no idea how encouraging it was for me to know that you will be on the stage to support me! I also want to thank Miranda and Marina for taking a good care of me and the whole BPE group. You are very good at looting the $\mathrm{Ph}$. D defense time/date.

To my Synthon fellows, Ciska, Jochem, Wanxun, Adrian, Eko, and Marjolein. Thank you all for the fantastic experience at Synthon. Adrian and Eko, the after work soccer time was a pure fun with you two! Marjolein, Adrian, and Jochem, my (Semi-) Wageningen pals, thank you for the many carpools. You've made the go-to-work trip less tiring and the back-to-home trip far more fascinating!

Thanks to my Chinese blokes and chicks: Lian Jie, Wen Caifang, Li Jing, Liu Qi, Wang Mengru, Fu Xing, Guan Aofei, Shi Quan, Wu Qu, Wang Si, Li Hequn, Liu Yinyin, Wang Weiliang, Zhang Jing, and all the members of the Team Dragon. You've kicked away my nostalgia and made my life in a foreign country so amiable.

In addition to those appreciations, some special "unacknowledgements" need to be granted. My special unacknowledgement goes to Yifan Zhu, who guided me into the whiskey world, which has resulted in so many unproductive writing nights. I also 
unacknowledge Xiangming, BJ, OJ, and Didi, who have put so much extra weight on me by continuous feeding me with foods (sometimes unhealthy "J" word foods). Unlike to hamsters, this effort did not offer any help to my productivity at all! But I do enjoy the time with Lai and most likely will with Jingyi, which makes up the sins you've done to me.

To this tiny lovely small city, Wageningen, you were my home for eight years. I am proud to have shared some important pages in my life with you. It was in this city that I met the most important other half of me, my wife Yimin, to whom I owe everything, to whom I want to pay with the rest of my life. Yimin, 相定于江湖, 不如 与你相濡以沫。

妈妈，爸谷感谢你们一直以来对我的支持，抱慊和你们距离这么远，这么久。

Muyun, you came to my life at the very end of this Ph.D journey. Thank you for pumping me with faith to accomplish this adventure, well, and I guess I have no option but to thank you for those sleepless nights.

See you, Wageningen and the Netherlands. See you, my friends and teachers. See you, my student life. 



\section{Curriculum Vitae}

Xiao Pan was born on 21 May 1988 in Yichang, China. He grew up and attended primary, middle, and high school in Yichang. In 2006, he moved to Nanjing, China to pursue his university studies in the Nanjing Forestry University. It was also in this university, he was firstly introduced to the biotechnology and bioengineering principles. Xiao obtained his engineering BSc diploma in 2010 with a dissertation entitled "Cultivation optimization and enzyme analysis of fungus Phanerochaete chrysosporium".

In the same year of 2010, Xiao moved abroad to the Netherlands, started to peruse his MSc study in Wageningen University \& Research. He was involved in a Master's program Biotechnology with a specialization in molecular and cellular biotechnology. In 2011, he worked in the Laboratory of Microbiology of Wageningen University \& Research on a metagenomics study for the sponge-microbe symbiosis under the supervision of Dr D. Sipkema and Prof. Dr H. Smidt. Xiao moved to Weesp, the Netherlands in 2012 to work on his internship at Feyecon Biotech BV. He studied the cultivation of micro and macro algae and extraction of growth hormone from algae for the production of commercial fertilizers. In August 2012, Xiao obtained his Master's degree from Wageningen University \& Research.

In October 2012, Xiao started working in Bioprocess Engineering group in Wageningen University \& Research as a PhD candidate on $\mathrm{CHO}$ cell culture process scale up/down. The PhD project was involved in a consortium comprises of Wageningen University, Synthon Biopharmaceuticals BV, Bioceros Holding BV, Biostream International BV, and IBIS Technologies BV, aiming to develop smart cultivation and screening technologies for accelerating early process development. His focus was to obtain a better understanding of cell physiology in fed-batch cultures and on the scale-down of these processes. The results of his research are presented in this thesis.

Currently, Xiao works as a principal scientist in FUJIFILM Diosynth biotechnologies in the United Kingdom, where he continues to seek for new challenges in the cell culture world. 


\section{List of Publications}

Pan, X., Streefland, M., Dalm, C., Wijffels, R. H. \& Martens, D. E. Selection of chemically defined media for $\mathrm{CHO}$ cell fed-batch culture processes. Cytotechnology 69, 39-56 (2017).

Pan, X., Dalm, C., Wijffels, R. H. \& Martens, D. E. Metabolic characterization of a $\mathrm{CHO}$ cell size increase phase in fed-batch cultures. Appl. Microbiol. Biotechnol. 101, 8101-8113 (2017).

Pan, X., Alsayyari, A. A., Dalm, C., Hageman, J. A., Wijffels, R. H. \& Martens, D. E. Transcriptome analysis of $\mathrm{CHO}$ cell size increase during a fed-batch process. Submitted for publication (2018).

Alsayyari, A. A.*, Pan, X.*, Dalm, C., van der Veen, J. W., Vriezen, N., Hageman, J. A., Wijffels, R. H. \& Martens, D. E. Transcriptome analysis for the scale-down of a CHO cell fed-batch process. Submitted for publication (2018).

* Both authors contributed equally to this publication 


\section{Overview of completed training activities}

\section{Discipline specific activities}

ESACT meeting (Lille, France, 2013) ${ }^{1}$

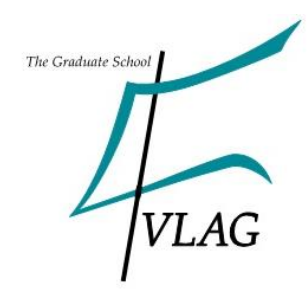

Comsol Multiphysics (COMSOL workshop, Zwolle, the Netherlands, 2013)

NBC Mini-symposium animal cell culture (Bilthoven, the Netherlands, 2013)

NBC-13 (Ede, the Netherlands, 2014) ${ }^{1}$

CAMO workshops (Nijmegen, the Netherlands, 2014)

Bioprocess design (BPS-BSDL, Wageningen, the Netherlands, 2014)

NBC-15 (Ede, the Netherlands, 2015) ${ }^{1}$

ESACT meeting (Barcelona, Spain, 2015) ${ }^{1}$

BPI international congress (Vienna, Austria, 2016) ${ }^{1}$

NBC-16 (Wageningen, the Netherlands, 2016) ${ }^{1}$

\section{General courses}

VLAG PhD week (Baarlo, the Netherlands, 2012)

PhD competence assessments (Wageningen, the Netherlands, 2012)

Project and time management (Wageningen, the Netherlands, 2014)

Scientific writing (Wageningen, the Netherlands, 2014)

Techniques for Writing and Presenting a Scientific Paper (Wageningen, the Netherlands, 2016)

Applied statistics (Wageningen, the Netherlands, 2016)

Multivariate analysis (Wageningen, the Netherlands, 2016)

PhD Workshop Carousel (Wageningen, the Netherlands, 2015)

\section{Optional activities}

Preparation of research proposal (2012)

BPE International excursion (Portugal, 2014) 1,2

PhD brainstorm days (2012, 2013 2, 2014, 2015, 2016 ²)

MSc thesis presentation and group meeting (2013-2017)

${ }^{1}$ poster presentation; ${ }^{2}$ oral presentation 
This thesis was financially supported by the EFRO Province of Gelderland and Overijssel, the Netherlands. The research in this thesis was performed at Bioprocess Engineering, Wageningen University, the Netherlands and the Upstream Development department of Synthon Biopharmaceuticals BV, Nijmegen, the Netherlands.

\section{Cover design by}

Venus Chen

Printed by

ProefschriftMaken II DigiForce 



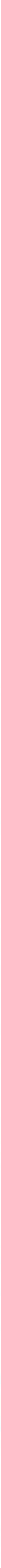